\title{
Le Système
}

\section{international d'unités}

The International

System

of Units

$8^{e}$ édition

2006

Bureau

international

des poids

et mesures

Organisation

intergouvernementale de la Convention

du Mètre 



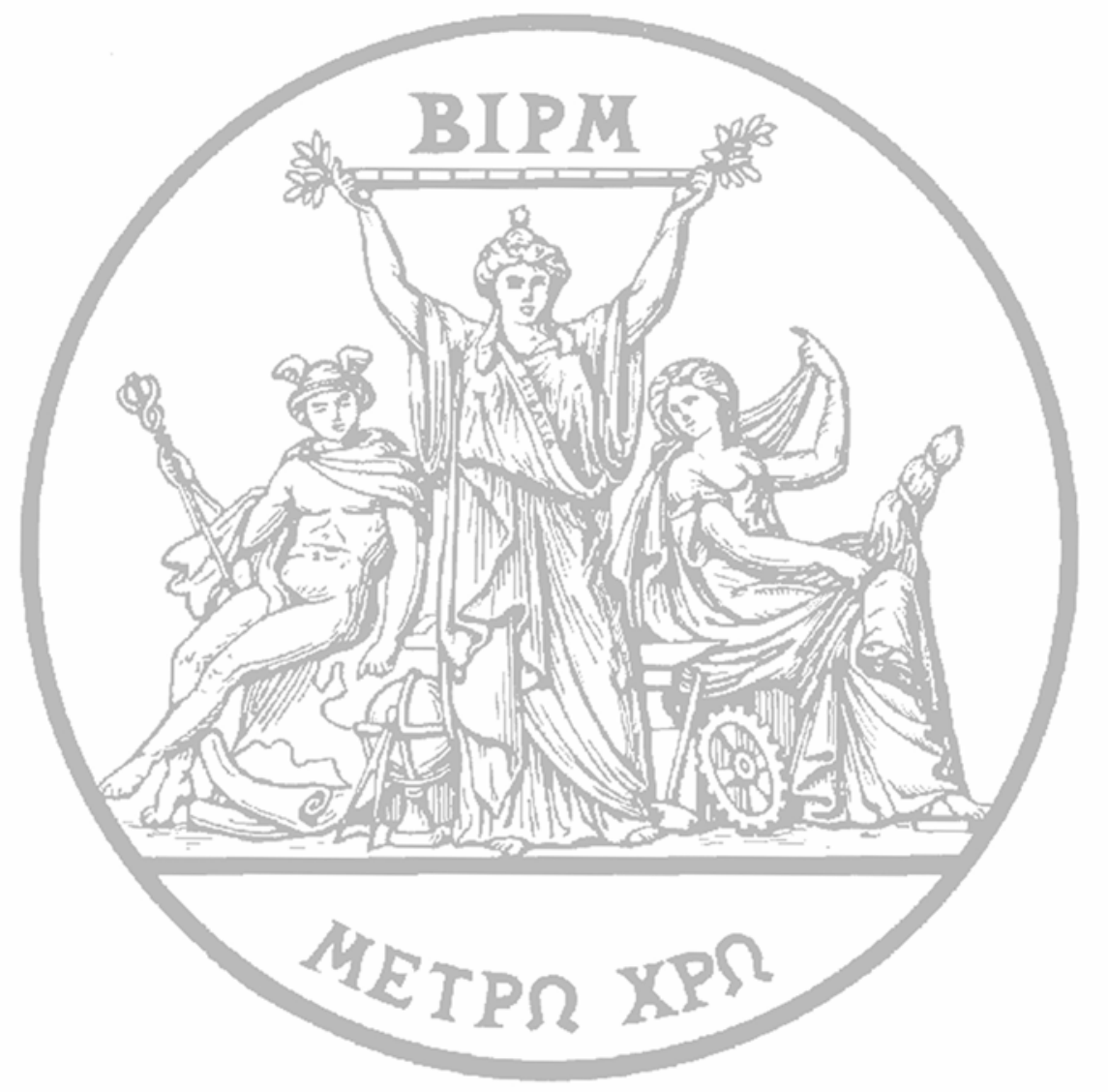



Le Système international

d'unités (SI)

English version

The International

System of Units (SI) 

Bureau international

des poids et mesures

\section{Le Système international d'unités (SI)}


Note sur l'utilisation du texte anglais (voir page 93)

Afin de mieux faire connaître ses travaux, le Comité international des poids et mesures publie une version en anglais de ses documents. Le lecteur doit cependant noter que le texte officiel est toujours celui qui est rédigé en français. C'est celui-ci qui fait autorité si une référence est nécessaire ou s'il y a doute sur l'interprétation.

Des traductions complètes ou partielles de cette brochure (ou de ses précédentes éditions) sont publiées en diverses langues, notamment en allemand, anglais, bulgare, chinois, coréen, espagnol, japonais, portugais, roumain, tchèque. L'ISO et de nombreux pays ont aussi publié des guides pour l'emploi des unités SI.

Édité par le BIPM,

Pavillon de Breteuil,

F-92312 Sèvres Cedex

France

Imprimé par : STEDI Media, Paris

ISBN 92-822-2213-6 


\section{Le BIPM et la Convention du Mètre}

Le Bureau international des poids et mesures (BIPM) a été créé par la Convention du Mètre signée à Paris le 20 mai 1875 par dix-sept États, lors de la dernière séance de la Conférence diplomatique du Mètre. Cette Convention a été modifiée en 1921.

Le Bureau international a son siège près de Paris, dans le domaine $\left(43520 \mathrm{~m}^{2}\right) \mathrm{du}$ Pavillon de Breteuil (Parc de Saint-Cloud) mis à sa disposition par le Gouvernement français; son entretien est assuré à frais communs par les États membres de la Convention du Mètre.

Le Bureau international a pour mission d'assurer l'unification mondiale des mesures ; il est donc chargé :

- d'établir les étalons fondamentaux et les échelles pour la mesure des principales grandeurs physiques et de conserver les prototypes internationaux ;

- d'effectuer la comparaison des étalons nationaux et internationaux ;

- d'assurer la coordination des techniques de mesure correspondantes ;

- d'effectuer et de coordonner les mesures des constantes physiques fondamentales qui interviennent dans les activités ci-dessus.

Le Bureau international fonctionne sous la surveillance exclusive du Comité international des poids et mesures (CIPM), placé lui-même sous l'autorité de la Conférence générale des poids et mesures (CGPM) à laquelle il présente son rapport sur les travaux accomplis par le Bureau international.

La Conférence générale rassemble des délégués de tous les États membres de la Convention du Mètre et se réunit actuellement tous les quatre ans dans le but :

- de discuter et de provoquer les mesures nécessaires pour assurer la propagation et le perfectionnement du Système international d'unités (SI), forme moderne du Système métrique ;

- de sanctionner les résultats des nouvelles déterminations métrologiques fondamentales et d'adopter diverses résolutions scientifiques de portée internationale ;

- d'adopter toutes les décisions importantes concernant la dotation, l'organisation et le développement du Bureau international.

Le Comité international comprend dix-huit membres appartenant à des États différents ; il se réunit actuellement tous les ans. Le bureau de ce Comité adresse aux Gouvernements des États membres de la Convention du Mètre un rapport annuel sur la situation administrative et financière du Bureau international. La principale mission du Comité international est d'assurer l'unification mondiale des unités de mesure, en agissant directement, ou en soumettant des propositions à la Conférence générale.
Au 31 décembre 2005, cinquante et un États étaient membres de cette Convention : Afrique du Sud, Allemagne, Argentine, Australie, Autriche, Belgique, Brésil, Bulgarie, Cameroun, Canada, Chili, Chine, Corée (Rép. de), Corée (Rép. pop. dém. de), Danemark, Dominicaine

(Rép.), Égypte, Espagne, États-Unis, Finlande, France, Grèce, Hongrie, Inde, Indonésie, Iran, Irlande, Israël, Italie, Japon, Malaisie, Mexique, Norvège, NouvelleZélande, Pakistan, PaysBas, Pologne, Portugal, Roumanie, Royaume-Uni, Russie (Féd. de), Serbie-etMonténégro, Singapour,

Slovaquie, Suède, Suisse, Tchèque (Rép.), Thaïlande, Turquie, Uruguay,

Venezuela.

Vingt États et entités économiques sont Associés à la Conférence générale : Bélarus, CARICOM, Costa Rica, Croatie, Cuba,

Équateur, Estonie, Hong Kong (Chine), Jamaïque, Kazakhstan, Kenya, Lettonie, Lituanie, Malte, Panama, Philippines, Slovénie, Taipei chinois, Ukraine, Viet Nam. 
Limitées à l'origine aux mesures de longueur et de masse et aux études métrologiques en relation avec ces grandeurs, les activités du Bureau international ont été étendues aux étalons de mesure électriques (1927), photométriques et radiométriques (1937), des rayonnements ionisants (1960), aux échelles de temps (1988) et à la chimie (2000). Dans ce but, un agrandissement des premiers laboratoires construits en 18761878 a eu lieu en 1929 ; de nouveaux bâtiments ont été construits en 1963-1964 pour les laboratoires de la section des rayonnements ionisants, en 1984 pour le travail sur les lasers, en 1988 pour la bibliothèque et des bureaux, et en 2001 a été inauguré un bâtiment pour l'atelier, des bureaux et des salles de réunion.

Environ quarante-cinq physiciens et techniciens travaillent dans les laboratoires du Bureau international. Ils y font principalement des recherches métrologiques, des comparaisons internationales des réalisations des unités et des vérifications d'étalons. Ces travaux font l'objet d'un rapport annuel détaillé qui est publié dans le Rapport $d u$ directeur sur l'activité et la gestion du Bureau international des poids et mesures.

Devant l'extension des tâches confiées au Bureau international en 1927, le Comité international a institué, sous le nom de Comités consultatifs, des organes destinés à le renseigner sur les questions qu'il soumet, pour avis, à leur examen. Ces Comités consultatifs, qui peuvent créer des groupes de travail temporaires ou permanents pour l'étude de sujets particuliers, sont chargés de coordonner les travaux internationaux effectués dans leurs domaines respectifs et de proposer au Comité international des recommandations concernant les unités.

Les Comités consultatifs ont un règlement commun (BIPM Proc.verb. Com. int. poids et mesures, 1963, 31, 97). Ils tiennent leurs sessions à des intervalles irréguliers. Le président de chaque Comité consultatif est désigné par le Comité international; il est généralement membre du Comité international. Les Comités consultatifs ont pour membres des laboratoires de métrologie et des instituts spécialisés, dont la liste est établie par le Comité international, qui envoient des délégués de leur choix. Ils comprennent aussi des membres nominativement désignés par le Comité international, et un représentant du Bureau international (Critères pour être membre des Comités consultatifs, BIPM Proc.-verb. Com. int. poids et mesures, 1996, 64, 6). Ces Comités sont actuellement au nombre de dix :

1. Le Comité consultatif d'électricité et magnétisme (CCEM), nouveau nom donné en 1997 au Comité consultatif d'électricité (CCE) créé en 1927 ;

2. Le Comité consultatif de photométrie et radiométrie (CCPR), nouveau nom donné en 1971 au Comité consultatif de photométrie (CCP) créé en 1933 (de 1930 à 1933 le CCE s'est occupé des questions de photométrie);

3. Le Comité consultatif de thermométrie (CCT), créé en 1937 ;

4. Le Comité consultatif des longueurs (CCL), nouveau nom donné en 1997 au Comité consultatif pour la définition du mètre (CCDM) créé en 1952;

5. Le Comité consultatif du temps et des fréquences (CCTF), nouveau nom donné en 1997 au Comité consultatif pour la définition de la seconde (CCDS) créé en 1956 ;

6. Le Comité consultatif des rayonnements ionisants (CCRI), nouveau nom donné en 1997 au Comité consultatif pour les étalons de mesure des rayonnements ionisants (CCEMRI) créé en 1958 (en 1969, ce Comité consultatif a institué 
quatre sections: Section I (Rayons x et $\gamma$, électrons), Section II (Mesure des radionucléides), Section III (Mesures neutroniques), Section IV (Étalons d'énergie $\alpha$ ); cette dernière section a été dissoute en 1975, son domaine d'activité étant confié à la Section II);

7. Le Comité consultatif des unités (CCU), créé en 1964 (ce Comité consultatif a remplacé la «Commission du système d'unités» instituée par le Comité international en 1954);

8. Le Comité consultatif pour la masse et les grandeurs apparentées (CCM), créé en 1980 ;

9. Le Comité consultatif pour la quantité de matière : métrologie en chimie (CCQM), créé en 1993 ;

10. Le Comité consultatif de l'acoustique, des ultrasons et des vibrations (CCAUV), créé en 1999.

Les travaux de la Conférence générale et du Comité international sont publiés par les soins du Bureau international dans les collections suivantes :

- Comptes rendus des séances de la Conférence générale des poids et mesures ;

- Procès-verbaux des séances du Comité international des poids et mesures.

Le Comité international a décidé en 2003 que les rapports des sessions des Comités consultatifs ne seraient plus imprimés, mais placés sur le site Web du BIPM, dans leur langue originale.

Le Bureau international publie aussi des monographies sur des sujets métrologiques particuliers et, sous le titre Le Système international d'unités (SI), une brochure remise à jour périodiquement qui rassemble toutes les décisions et recommandations concernant les unités.

La collection des Travaux et mémoires du Bureau international des poids et mesures (22 tomes publiés de 1881 à 1966) a été arrêtée par décision du Comité international, de même que le Recueil de travaux du Bureau international des poids et mesures (11 volumes publiés de 1966 à 1988).

Les travaux du Bureau international font l'objet de publications dans des journaux scientifiques ; une liste en est donnée chaque année dans le Rapport du directeur sur l'activité et la gestion du Bureau international des poids et mesures.

Depuis 1965 la revue internationale Metrologia, éditée sous les auspices du Comité international des poids et mesures, publie des articles sur la métrologie scientifique, sur l'amélioration des méthodes de mesure, les travaux sur les étalons et sur les unités, ainsi que des rapports concernant les activités, les décisions et les recommandations des organes de la Convention du Mètre. 
1 Introduction 13

1.1 Grandeurs et unités $\quad 13$

1.2 Le Système international d'unités (SI) et le système de grandeurs
correspondant

$\begin{array}{lll}1.3 & \text { Dimension des grandeurs } & 15\end{array}$

1.4 Unités cohérentes, unités dérivées ayant des noms spéciaux et préfixes SI 16

$\begin{array}{lll}1.5 & \text { Les unités SI dans le cadre de la relativité générale } & 17\end{array}$

$\begin{array}{lll}1.6 & \text { Unités des grandeurs décrivant des effets biologiques } & 17\end{array}$

$\begin{array}{lll}1.7 & \text { Législation sur les unités } & 18\end{array}$

$\begin{array}{lll}1.8 \text { Note historique } & 18\end{array}$

2 Unités SI $\quad 21$

2.1 Unités SI de base $\quad \mathbf{2 1}$

2.1.1 Définitions $\quad 21$

2.1.1.1 Unité de longueur (mètre) 22

2.1.1.2 Unité de masse (kilogramme) 22

2.1.1.3 Unité de temps (seconde) 22

2.1.1.4 Unité de courant électrique (ampère) 23

2.1.1.5 Unité de température thermodynamique (kelvin) 23

2.1.1.6 Unité de quantité de matière (mole) $\quad \mathbf{2 4}$

2.1.1.7 Unité d'intensité lumineuse (candela) 26

2.1.2 Symboles des sept unités de base $\quad 26$

2.2 Unités SI dérivées $\quad 27$

2.2.1 Unités dérivées exprimées à partir des unités de base 27

2.2.2 Unités ayant des noms spéciaux et des symboles particuliers ; unités faisant appel à des noms spéciaux et des symboles particuliers $\quad 27$

2.2.3 Unités des grandeurs sans dimension, aussi désignées $\begin{array}{ll}\text { grandeurs de dimension un } & \mathbf{3 0}\end{array}$

3 Multiples et sous-multiples décimaux des unités SI 32

3.1 Préfixes SI $\quad 32$

3.2 Le kilogramme $\quad 33$ 
4.1 Unités en dehors du SI en usage avec le SI et unités fondées sur des constantes fondamentales

4.2 Autres unités en dehors du SI dont l'usage n'est pas recommandé

5 Règles d'écriture des noms et symboles d'unités et expression des valeurs des grandeurs

5.1 Symboles des unités

5.2 Noms des unités

5.3 Règles et conventions stylistiques servant à exprimer les valeurs des grandeurs

5.3.1 Valeur et valeur numérique d'une grandeur ; utilisation du calcul formel

5.3.2 Symboles des grandeurs et des unités

5.3.3 Écriture de la valeur d'une grandeur

5.3.4 Écriture des nombres et séparateur décimal

5.3.5 Expression de l'incertitude de mesure associée à la valeur d'une grandeur

5.3.6 Multiplication ou division des symboles des grandeurs, des valeurs des grandeurs et des nombres

5.3.7 Valeur des grandeurs sans dimension, ou grandeurs de dimension un

Annexe 1. - Décisions de la Conférence générale des poids et mesures et du Comité international des poids et mesures

Annexe 2. - Réalisation pratique des définitions des principales unités

Annexe 3. - Unités pour la mesure des grandeurs photochimiques et photobiologiques 



\section{Préface à la $8^{\mathrm{e}}$ édition}

Nous avons le plaisir d'introduire la $8^{\mathrm{e}}$ édition de cette publication, communément appelée Brochure sur le SI, qui définit et présente le Système international d'unités, le SI. Cette brochure est publiée en version papier; elle est aussi disponible sous forme électronique sur le site Internet du BIPM à l'adresse www.bipm.org/fr/si/si brochure/. Depuis 1970, le Bureau international des poids et mesures, le BIPM, a déjà publié sept éditions de ce document. Son but principal est de définir et promouvoir le SI, qui est utilisé dans le monde entier comme langage préféré dans les domaines des sciences et de la technologie, depuis son adoption en 1948 par la $9^{\text {e }}$ Conférence générale des poids et mesures, la CGPM.

Le SI est, bien sûr, un système évolutif, qui reflète les meilleures pratiques en matière de mesure du moment. Cette $8^{\mathrm{e}}$ édition contient donc un certain nombre de changements par rapport à la précédente édition. Comme auparavant, elle donne les définitions de toutes les unités de base, ainsi que les Résolutions et Recommandations de la Conférence générale des poids et mesures et du Comité international des poids et mesures (CIPM) concernant le Système international d'unités. Les références officielles de ces décisions se trouvent dans les volumes successifs des Comptes rendus de la Conférence générale (CR) et des Procès-verbaux du Comité international $(\mathrm{PV})$; un grand nombre d'entre elles ont aussi été publiées dans Metrologia. Pour simplifier l'utilisation pratique du Système international, le texte fournit des explications à propos de ces décisions, et le premier chapitre présente une introduction générale sur l'établissement des systèmes d'unités et du SI en particulier. Les définitions et réalisations de toutes les unités sont aussi examinées dans le contexte de la relativité générale. Pour la première fois, cette brochure comporte aussi une brève discussion des unités associées aux grandeurs biologiques.

L'annexe 1 reproduit, par ordre chronologique, toutes les décisions (Résolutions, Recommandations, déclarations) promulguées depuis 1889 par la Conférence générale et par le Comité international sur les unités de mesure et le Système international d'unités.

L'annexe 2 existe uniquement sous forme électronique et est accessible à l'adresse www.bipm.org/fr/si/si brochure/appendix 2/. Elle met l'accent sur la réalisation pratique de certaines unités importantes, en accord avec les définitions données dans le texte principal. Ceci est utile aux laboratoires de métrologie pour réaliser les unités physiques et pour étalonner les étalons matériels et les instruments de mesure de la plus haute qualité. Cette annexe sera mise à jour régulièrement pour refléter les progrès des techniques expérimentales utilisées pour réaliser les unités.

L'annexe 3 présente les unités utilisées pour la mesure des effets actiniques dans les matériaux biologiques. 
Le Comité consultatif des unités du CIPM, le CCU, est responsable de la préparation de ce document; le CCU et le CIPM ont tous deux approuvé le texte final. Cette $8^{\mathrm{e}}$ édition est une révision de la $7^{\mathrm{e}}$ édition (1998); elle prend en compte les décisions de la Conférence générale et du Comité international depuis la publication de la $7^{\mathrm{e}}$ édition.

Depuis plus de trente-cinq ans, ce document est utilisé comme ouvrage de référence dans de nombreux pays, organisations et unions scientifiques. Afin de rendre son contenu accessible à un plus grand nombre d'utilisateurs, le Comité international a décidé, en 1985, de publier une version en anglais du texte de la $5^{\mathrm{e}}$ édition; les éditions ultérieures ont conservé cette présentation bilingue. Pour la première édition en anglais, le BIPM s'est efforcé de produire une traduction fiable du texte original en collaboration étroite avec le National Physical Laboratory (Teddington, RoyaumeUni) et avec le National Institute of Standards and Technology (Gaithersburg, ÉtatsUnis), qui s'appelait à l'époque le National Bureau of Standards. Dans la présente édition, les versions française et anglaise ont été préparées par le CCU en collaboration étroite avec le BIPM.

La $22^{\circ}$ Conférence générale a décidé, en 2003 , de suivre la décision prise par le Comité international en 1997 : «le symbole du séparateur décimal pourra être soit le point sur la ligne, soit la virgule sur la ligne. » $\mathrm{D}$ 'après cette décision, et suivant l'usage dans les deux langues, on utilise dans cette édition le point sur la ligne comme séparateur décimal en anglais, et la virgule sur la ligne en français. Cette pratique n'a aucune implication en ce qui concerne la traduction du séparateur décimal dans les autres langues. Il faut noter qu'il existe de petites variations dans l'orthographe de certains mots en anglais (par exemple, «metre» et «meter », «litre » et «liter »). Le texte anglais publié ici suit la norme ISO 31, Grandeurs et unités.

Le lecteur doit cependant noter que le texte officiel est celui qui est rédigé en français. C'est le texte français qui fait autorité si une référence est nécessaire ou s'il y a un doute sur l'interprétation.

Mars 2006

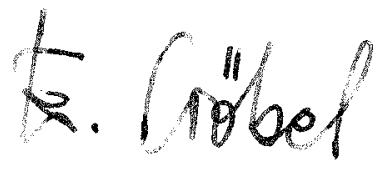

E. Göbel

Président du CIPM

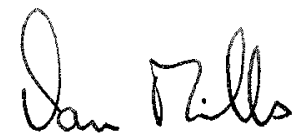

I. M. Mills

Président du $C C U$
Antrew hacland

A. J. Wallard

Directeur du BIPM 


\section{Introduction}

\section{$1.1 \quad$ Grandeurs et unités}

La valeur d'une grandeur est généralement exprimée sous la forme du produit d'un nombre par une unité. L'unité n'est qu'un exemple particulier de la grandeur concernée, utilisé comme référence. Le nombre est le rapport entre la valeur de la grandeur en question et l'unité. Pour une grandeur particulière, on peut utiliser de nombreuses unités différentes. Par exemple, la vitesse $v$ d'une particule peut être exprimée sous la forme $v=25 \mathrm{~m} / \mathrm{s}=90 \mathrm{~km} / \mathrm{h}$, les unités mètre par seconde et kilomètre par heure étant des unités alternatives pour exprimer la même valeur de la grandeur « vitesse ». Cependant, comme il est important de disposer d'un ensemble d'unités bien définies, universellement reconnues et faciles à utiliser pour la multitude des mesures qui confortent l'assise de notre société, les unités choisies doivent être accessibles à tous, supposées constantes dans le temps et l'espace, et faciles à réaliser avec une exactitude élevée.

Pour établir un système d'unités, comme le Système international d'unités, le SI, il est nécessaire tout d'abord d'établir un système de grandeurs et une série d'équations définissant les relations entre ces grandeurs. Ceci est nécessaire parce que les équations reliant les grandeurs entre elles déterminent celles reliant les unités entre elles, comme décrit dans la suite de ce document. Il est commode aussi de choisir les définitions d'un nombre restreint d'unités que nous appelons les unités de base, et de définir ensuite les unités des autres grandeurs comme produits de puissances des unités de base, que nous appelons les unités dérivées. De manière similaire, les grandeurs correspondantes sont décrites comme grandeurs de base et grandeurs dérivées, et les équations donnant les grandeurs dérivées en fonction des grandeurs de base sont utilisées pour exprimer les unités dérivées en fonction des unités de base (voir 1.4 ci-dessous). Il est donc logique que le choix des grandeurs et des équations reliant les grandeurs précède celui des unités.

Du point de vue scientifique, la division des grandeurs en grandeurs de base et grandeurs dérivées est affaire de convention; ce n'est pas fondamental pour la compréhension de la physique sous-jacente. Toutefois, pour ce qui concerne les unités, il est important que la définition de chaque unité de base soit effectuée avec un soin particulier, afin de satisfaire aux exigences mentionnées au premier paragraphe ci-dessus, puisqu'elles assurent le fondement du système d'unités tout entier. La définition des unités dérivées en fonction des unités de base découle des équations définissant les grandeurs dérivées en fonction des grandeurs de base. Ainsi l'établissement d'un système d'unités, qui constitue le sujet de cette brochure, est intimement lié aux équations algébriques reliant les grandeurs correspondantes.

Le nombre de grandeurs dérivées importantes pour la science et la technologie est bien sûr sans limite. Lorsque de nouveaux domaines scientifiques se développent, de nouvelles grandeurs sont introduites par les chercheurs afin de représenter les propriétés du domaine, et ces nouvelles grandeurs engendrent de nouvelles équations
Les termes grandeur et unité sont définis dans le Vocabulaire international des termes fondamentaux et généraux de la métrologie, le VIM.

La grandeur vitesse, $v$, peut être exprimée en fonction des grandeurs distance parcourue, $x$, et durée du parcours, $t$, au moyen de l'équation $v=\mathrm{d} x / \mathrm{d} t$.

Dans la plupart des systèmes de grandeurs et d'unités, la distance $x$ et le temps $t$ sont considérés comme des grandeurs de base, pour lesquelles on peut choisir comme unités de base le mètre (symbole $\mathrm{m}$ ) et la seconde (symbole s). La vitesse $v$ est ainsi considérée comme une grandeur dérivée, dont l'unité est le mètre par seconde (symbole m/s).

Par exemple, en

électrochimie, la mobilité électrique d'un ion, $u$, est définie comme le rapport entre sa vitesse $v$ et l'intensité du champ électrique $E: u=v / E$. L'unité de mobilité électrique est donc donnée sous la forme : $(\mathrm{m} / \mathrm{s}) /(\mathrm{V} / \mathrm{m})=\mathrm{m}^{2} \mathrm{~V}^{-1} \mathrm{~s}^{-1}$, et peut être aisément reliée aux unités de base choisies (V est le symbole du volt, unité dérivée du SI). 
afin de les relier aux grandeurs familières, puis aux grandeurs de base. Ainsi les unités dérivées à utiliser avec ces nouvelles grandeurs peuvent être définies comme le produit de puissances d'unités de base choisies précédemment.

\subsection{Le Système international d'unités (SI) et le système de grandeurs correspondant}

Cette brochure a pour objet de présenter les informations nécessaires à la définition et à l'utilisation du Système international d'unités, universellement connu sous l'abréviation SI. Le SI a été établi et défini par la Conférence générale des poids et mesures, la CGPM (voir la Note historique section 1.8 ci-dessous)*.

Le système de grandeurs à utiliser avec le SI, y compris les équations reliant ces grandeurs entre elles, correspond en fait aux grandeurs et équations de la physique, bien connues de tous les scientifiques, techniciens et ingénieurs. Elles figurent dans tous les manuels et dans de nombreuses publications de référence, mais toute liste ne constitue qu'une sélection parmi les grandeurs et équations existantes, lesquelles sont en nombre illimité. Un grand nombre de grandeurs, leurs noms et symboles recommandés et les équations les reliant les unes aux autres, sont mentionnés dans les normes internationales ISO 31 et CEI 60027 produites par le Comité technique 12 de l'Organisation internationale de normalisation, l'ISO/TC 12, et par le Comité technique 25 de la Commission électrotechnique internationale, CEI/TC 25. Les normes ISO 31 et CEI 60027 sont en cours de révision par ces deux organisations de normalisation, qui y travaillent conjointement. La norme révisée par ces deux organisations constituera la norme ISO/CEI 80000, Grandeurs et unités, dans laquelle il est proposé que l'ensemble des grandeurs et équations utilisées avec le SI soit désigné sous le nom de Système international de grandeurs.

Les grandeurs de base utilisées dans le SI sont la longueur, la masse, le temps, le courant électrique, la température thermodynamique, la quantité de matière et l'intensité lumineuse. Les grandeurs de base sont, par convention, considérées comme indépendantes. Les unités de base correspondantes du SI, choisies par la CGPM, sont le mètre, le kilogramme, la seconde, l'ampère, le kelvin, la mole et la candela. Les définitions de ces unités de base sont données dans la section 2.1.1 au chapitre suivant. Les unités dérivées du SI sont ensuite formées des produits de puissances des unités de base, selon les relations algébriques qui définissent les grandeurs dérivées correspondantes en fonction des grandeurs de base (voir 1.4 ci-dessous).

À de rares occasions, on a le choix entre plusieurs formes de relations entre les grandeurs. Un exemple particulièrement important concerne la définition des grandeurs électromagnétiques. Les équations électromagnétiques rationalisées à quatre grandeurs, utilisées avec le SI, sont fondées sur la longueur, la masse, le temps et le courant électrique. Dans ces équations, la constante électrique $\varepsilon_{0}$ (la permittivité du vide) et la constante magnétique $\mu_{0}$ (la perméabilité du vide) ont des dimensions et des valeurs qui vérifient l'équation $\varepsilon_{0} \mu_{0}=1 / c_{0}^{2}$, où $c_{0}$ est la vitesse de la lumière dans le vide. La loi de Coulomb décrit la force électrostatique entre deux particules de charges $q_{1}$ et $q_{2}$ à une distance $r$ sous la forme** :

* Les sigles utilisés dans cette brochure et leur signification figurent à la page 87 .

** Les vecteurs sont exprimés par des symboles en caractères gras. 


$$
\boldsymbol{F}=\frac{q_{1} q_{2} \boldsymbol{r}}{4 \pi \varepsilon_{0} r^{3}}
$$

et l'équation de la force magnétique s'exerçant entre deux éléments de fils électriques minces parcourus par des courants électriques, $i_{1} \mathrm{~d} l_{1}$ and $i_{2} \mathrm{~d} l_{2}$, est exprimée sous la forme suivante :

$$
\mathrm{d}^{2} \boldsymbol{F}=\frac{\mu_{0}}{4 \pi} \frac{i_{1} \mathrm{~d} \boldsymbol{l}_{1} \times\left(i_{2} \mathrm{~d} \boldsymbol{l}_{2} \times \boldsymbol{r}\right)}{r^{3}}
$$

où $\mathrm{d}^{2} \boldsymbol{F}$ est la différentielle seconde de la force $\boldsymbol{F}$. Ces équations, sur lesquelles le SI est fondé, sont différentes de celles utilisées dans les systèmes CGS-UES, CGS-UEM et CGS de Gauss, dans lesquelles $\varepsilon_{0}$ et $\mu_{0}$ sont des grandeurs sans dimension, choisies comme étant égales à un, et où les facteurs de rationalisation $4 \pi$ sont omis.

\subsection{Dimension des grandeurs}

Par convention, les grandeurs physiques sont organisées selon un système de dimensions. Chacune des sept grandeurs de base du SI est supposée avoir sa propre dimension, représentée symboliquement par une seule lettre majuscule sans empattement en romain. Les symboles utilisés pour les grandeurs de base, et les symboles utilisés pour indiquer leur dimension, sont les suivants :

Grandeurs de base et dimensions utilisées avec le SI

\begin{tabular}{lll}
\hline Grandeur de base & Symbole de la grandeur & Symbole de la dimension \\
\hline longueur & $l, x, r$, etc. & $\mathrm{L}$ \\
masse & $m$ & $\mathrm{M}$ \\
temps, durée & $t$ & $\mathrm{~T}$ \\
courant électrique & $I, i$ & $\mathrm{I}$ \\
température thermodynamique & $T$ & $\Theta$ \\
quantité de matière & $n$ & $\mathrm{~N}$ \\
intensité lumineuse & $I_{\mathrm{v}}$ & $\mathrm{J}$
\end{tabular}

Toutes les autres grandeurs sont des grandeurs dérivées, qui peuvent être exprimées en fonction des grandeurs de base à l'aide des équations de la physique. Les dimensions des grandeurs dérivées sont écrites sous la forme de produits de puissances des dimensions des grandeurs de base au moyen des équations qui relient les grandeurs dérivées aux grandeurs de base. En général la dimension d'une grandeur $Q$ s'écrit sous la forme d'un produit dimensionnel,

$$
\operatorname{dim} Q=\mathrm{L}^{\alpha} \mathbf{M}^{\beta} \mathbf{T}^{\gamma} \mathbf{I}^{\delta} \Theta^{\varepsilon} \mathbf{N}^{\zeta} \mathbf{J}^{\eta}
$$

où les exposants $\alpha, \beta, \gamma, \delta, \varepsilon, \zeta$ et $\eta$, qui sont en général de petits nombres entiers, positifs, négatifs ou nuls, sont appelés exposants dimensionnels. L'information fournie par la dimension d'une grandeur dérivée sur la relation entre cette grandeur et les grandeurs de base est la même que celle contenue dans l'unité SI pour la grandeur dérivée, elle-même obtenue comme produit de puissances des unités de base du SI.

Les symboles des grandeurs sont toujours écrits en italique alors que les symboles des dimensions sont écrits en majuscules sans empattement en romain.

Pour certaines grandeurs, il est possible d'utiliser différents symboles, comme indiqué pour la longueur ou le courant électrique.

Notons que les symboles donnés pour les grandeurs ne sont que recommandés.

Par contre, les symboles donnés pour les unités dans cette brochure, ainsi que leur style et leur forme, sont ceux qui doivent être obligatoirement utilisés (voir chapitre 5).

Les symboles des dimensions et les exposants sont traités selon les règles ordinaires de l'algèbre. Par exemple, la dimension pour la superficie s'écrit $L^{2}$; la dimension pour la vitesse $\mathrm{LT}^{-1}$; la dimension pour la force $\mathrm{LMT}^{-2}$; et la dimension pour l'énergie $\mathrm{L}^{2} \mathrm{MT}^{-2}$. 
Certaines grandeurs dérivées $Q$ sont définies par une équation aux grandeurs telle que tous les exposants dimensionnels entrant dans l'expression de la dimension de $Q$ sont égaux à zéro. C'est vrai, en particulier, pour une grandeur définie comme le rapport entre deux grandeurs de même nature. Ces grandeurs sont décrites comme étant sans dimension, ou de dimension un. L'unité cohérente dérivée de telles grandeurs est toujours le nombre un, 1, puisque c'est le rapport entre les unités de deux grandeurs de même nature, donc identiques.

Il existe également des grandeurs qui ne peuvent pas être décrites au moyen des sept grandeurs de base du SI, mais dont la valeur est déterminée par comptage. Par exemple le nombre de molécules, la dégénérescence en mécanique quantique (le nombre d'états indépendants ayant la même énergie) et la fonction de partition en thermodynamique statistique (le nombre d'états thermiques accessibles). Ces grandeurs sont aussi habituellement considérées comme sans dimension, ou de dimension un, et ont pour unité le nombre un, 1.

\subsection{Unités cohérentes, unités dérivées ayant des noms spéciaux et préfixes SI}

Les unités dérivées sont définies comme le produit de puissances des unités de base. Quand le produit des puissances ne comprend pas de facteur numérique autre que 1, les unités dérivées sont appelées unités dérivées cohérentes. Les unités de base et les unités dérivées cohérentes du SI forment un ensemble cohérent, désigné sous le nom d'ensemble cohérent des unités SI. Le mot cohérent est utilisé ici dans le sens suivant : lorsque l'on utilise des unités cohérentes, les équations reliant les valeurs numériques des grandeurs prennent exactement la même forme que les équations reliant les grandeurs proprement dites. Ainsi, si l'on utilise uniquement des unités d'un ensemble cohérent, on n'a jamais besoin de facteurs de conversion entre les unités.

L'expression de l'unité cohérente d'une grandeur dérivée peut être obtenue à partir $\mathrm{du}$ produit dimensionnel de la grandeur en remplaçant le symbole de chaque dimension par le symbole de l'unité de base correspondante.

Certaines unités dérivées cohérentes du SI ont reçu des noms spéciaux, par souci de simplification (voir 2.2.2, p. 27). Il est important de souligner que chaque grandeur physique n'a qu'une seule unité SI cohérente, même si cette unité peut être exprimée sous différentes formes au moyen de noms spéciaux ou de symboles particuliers. L'inverse, toutefois, n'est pas vrai ; la même unité SI peut, dans certains cas, être employée pour exprimer les valeurs de plusieurs grandeurs différentes (voir p. 30).

La Conférence générale a, de plus, adopté une série de préfixes pour la formation des multiples et sous-multiples décimaux des unités SI cohérentes (voir 3.1, p. 32, la liste des noms de préfixes et de leur symbole). Ces préfixes sont commodes pour exprimer les valeurs de grandeurs beaucoup plus grandes ou beaucoup plus petites que l'unité cohérente. Suivant la Recommandation 1 (1969) du Comité international (voir p. 65), ces préfixes sont désignés sous le nom de préfixes SI. (Ces préfixes sont aussi parfois utilisés avec des unités en dehors du SI, comme décrit dans le chapitre 4 de cette brochure). Cependant, quand un préfixe est utilisé avec une unité du SI, l'unité dérivée obtenue n'est plus cohérente, car le préfixe introduit un facteur numérique différent de 1 dans l'expression de l'unité dérivée en fonction des unités de base.

Par dérogation à la règle, le nom du kilogramme, l'unité de base pour la masse, comprend le préfixe kilo, pour des raisons historiques. Il est néanmoins considéré
Par exemple, l'indice de réfraction d'un milieu est défini comme le rapport entre la vitesse de la lumière dans le vide et celle dans ce milieu ; c'est le rapport entre deux grandeurs de même nature. C'est donc une grandeur sans dimension.

D'autres exemples de grandeurs sans dimension sont : l'angle plan, la fraction massique, la permittivité relative, la perméabilité relative, et la finesse d'une cavité PerotFabry.

ar exemple, la combinaison particulière des unités de base $\mathrm{m}^{2} \mathrm{~kg} \mathrm{~s}^{-2}$ a reçu le nom spécial joule, symbole J, quand elle est l'unité d'une énergie. Par définition $\mathrm{J}=\mathrm{m}^{2} \mathrm{~kg} \mathrm{~s}^{-2}$.

Par commodité, la longueur d'une liaison chimique est plus généralement exprimée en nanomètres, nm, qu'en mètres, $\mathrm{m}$; et la distance entre Londres et Paris est généralement exprimée en kilomètres, km, plutôt qu'en mètres, $m$. 
comme une unité de base du SI. Les multiples et sous-multiples du kilogramme sont formés en attachant des noms de préfixes au nom de l'unité "gramme» et des symboles de préfixes au symbole d'unité «g » (voir 3.2, p. 33). Ainsi $10^{-6} \mathrm{~kg}$ s'écrit milligramme, $\mathrm{mg}$, et pas microkilogramme, $\mu \mathrm{kg}$.

L'ensemble des unités SI comprend l'ensemble des unités cohérentes et les multiples et sous-multiples de ces unités formés en les combinant aux préfixes SI. Il est désigné sous le nom d'ensemble complet des unités SI, ou simplement unités SI, ou unités du $S I$. Notons toutefois que les multiples et sous-multiples décimaux des unités du SI ne forment pas un ensemble cohérent.

Le mètre par seconde, symbole $\mathrm{m} / \mathrm{s}$, est l'unité SI cohérente de vitesse. Le kilomètre par seconde, $\mathrm{km} / \mathrm{s}$, le centimètre par seconde, $\mathrm{cm} / \mathrm{s}$, et le millimètre par seconde, $\mathrm{mm} / \mathrm{s}$, sont aussi des unités SI, mais ce ne sont pas des unités SI cohérentes.

\subsection{Les unités SI dans le cadre de la relativité générale}

Les définitions des unités de base du SI ont été adoptées dans un contexte qui ne tient pas compte des effets relativistes. Si l'on introduit une telle notion, il est clair que ces définitions ne s'appliquent que dans un petit domaine spatial qui accompagne dans leur mouvement les étalons qui les réalisent. Les unités de base du SI sont donc des unités propres; leurs réalisations proviennent d'expériences locales, dans lesquelles les effets relativistes à prendre en compte sont ceux de la relativité restreinte. Les constantes de la physique sont des grandeurs locales dont la valeur est exprimée en unités propres.

Les réalisations physiques de la définition d'une unité sont généralement comparées La question des unités propres est traitée dans la Résolution A4 adoptée par la $\mathrm{XXI}^{\mathrm{e}}$ Assemblée générale de l'Union astronomique internationale (UAI) en 1991 et dans le rapport du Groupe de travail du CCDS sur l'application de la relativité générale à la métrologie (Metrologia, au niveau local. Toutefois, pour les étalons de fréquence, il est possible d'effectuer de telles comparaisons à distance au moyen de signaux électromagnétiques. Pour interpréter les résultats, il est nécessaire de faire appel à la théorie de la relativité générale puisque celle-ci prédit, entre autres choses, un décalage de fréquence entre les étalons d'environ $1 \times 10^{-16}$ en valeur relative par mètre d'altitude à la surface de la Terre. Des effets de cet ordre de grandeur ne peuvent être négligés lors de la comparaison des meilleurs étalons de fréquence.

\subsection{Unités des grandeurs décrivant des effets biologiques}

Les unités des grandeurs décrivant des effets biologiques sont souvent difficiles à relier aux unités du SI parce qu'elles incluent habituellement des facteurs de pondération que l'on peut ne pas connaître ou définir avec précision, et qui peuvent dépendre à la fois de l'énergie et de la fréquence. Ces unités ne sont pas des unités du SI ; elles sont décrites brièvement dans cette section.

Les rayonnements optiques sont susceptibles de produire des modifications chimiques dans les matériaux vivants ou inertes. Cette propriété est appelée actinisme et les rayonnements capables de causer de tels changements sont connus sous le nom de rayonnements actiniques. Les résultats de mesure de certaines grandeurs photochimiques ou photobiologiques peuvent être exprimés en unités du SI. Cette question est discutée brièvement dans l'annexe 3 de cette brochure.

Le son cause de petites fluctuations de pression dans l'air, qui s'ajoutent à la pression atmosphérique normale, et qui sont perçues par l'oreille humaine. La sensibilité de l'oreille dépend de la fréquence sonore, et n'est pas une fonction simple de l'amplitude des variations de pression et de fréquence. Par conséquent, des grandeurs pondérées en fonction de la fréquence sont utilisées en acoustique pour donner une représentation approximative de la manière dont le son est perçu. Ces grandeurs sont 
employées par exemple dans les études sur la protection contre les dommages auditifs. Les effets des ondes acoustiques ultrasonores sont source de préoccupations similaires dans le diagnostic médical et dans le domaine thérapeutique.

Les rayonnements ionisants déposent de l'énergie dans la matière irradiée. Le rapport entre l'énergie déposée et la masse est appelé dose absorbée. Des doses élevées de rayonnements ionisants tuent les cellules. Ceci est utilisé en radiothérapie et des fonctions de pondération biologiques appropriées sont utilisées pour comparer les effets thérapeutiques de différents traitements. De faibles doses non mortelles peuvent causer des dommages aux organismes vivants, par exemple induire un cancer; ainsi les réglements relatifs à la radioprotection sont fondés sur des fonctions appropriées de pondération des risques pour les doses faibles.

Il existe une classe d'unités servant à quantifier l'activité biologique de certaines substances utilisées pour le diagnostic médical et la thérapie, qui ne peuvent pas encore être définies en fonction des unités du SI. En effet, le mécanisme de l'effet biologique spécifique qui donne à ces substances leur usage médical n'est pas encore suffisamment bien compris pour être quantifiable en fonction de paramètres physicochimiques. Compte tenu de leur importance pour la santé humaine et la sécurité, l'Organisation mondiale de la santé (OMS) a pris la responsabilité de définir des unités internationales OMS pour l'activité biologique de ces substances.

\section{$1.7 \quad$ Législation sur les unités}

Les États fixent, par voie législative, les règles concernant l'utilisation des unités sur le plan national, soit pour l'usage général, soit pour certains domaines particuliers comme le commerce, la santé, la sécurité publique ou l'enseignement. Dans la plupart des pays, la législation est fondée sur l'emploi du Système international d'unités.

L'Organisation internationale de métrologie légale (OIML), créée en 1955, s'occupe de l'harmonisation internationale de ces législations.

\section{$1.8 \quad$ Note historique}

Les paragraphes précédents de ce chapitre présentent brièvement comment un système d'unités, et le Système international d'unités en particulier, est établi. Cette note rend compte brièvement de l'évolution historique du Système international.

La $9^{\text {e }}$ CGPM (1948, Résolution 6 ; CR, 64), chargea le Comité international :

- d'étudier l'établissement d'une réglementation complète des unités de mesure ;

- d'ouvrir à cet effet une enquête officielle sur l'opinion des milieux scientifiques, techniques et pédagogiques de tous les pays et

- de faire des recommandations sur l'établissement d'un système pratique d'unités de mesure, susceptible d'être adopté par tous les pays signataires de la Convention du Mètre.

Cette même Conférence générale adopta aussi la Résolution 7 (CR, 70) qui fixe des principes généraux pour l'écriture des symboles d'unités et donna une liste de quelques unités cohérentes ayant un nom spécial.

La $10^{\mathrm{e}}$ CGPM (1954, Résolution 6; CR, 80) et la $14^{\mathrm{e}}$ CGPM (1971, Résolution 3 ; CR, 78 et Metrologia, 1972, 8, 36) adoptèrent comme unités de base de ce système pratique d'unités les unités des sept grandeurs suivantes : longueur, masse, temps, 
courant électrique, température thermodynamique, quantité de matière et intensité lumineuse.

La $11^{\mathrm{e}}$ CGPM (1960, Résolution 12 ; CR, 87) adopta le nom Système international d'unités, avec l'abréviation internationale SI, pour ce système pratique d'unités et fixa des règles pour les préfixes, les unités dérivées et les unités supplémentaires (disparues depuis), et d'autres indications, établissant ainsi une réglementation d'ensemble pour les unités de mesure. Au cours des réunions qui suivirent, la Conférence générale et le Comité international étoffèrent et modifièrent selon les besoins la structure originelle du SI pour tenir compte des progrès de la science et des besoins des utilisateurs.

Il est possible de résumer comme suit les principales étapes historiques qui conduisirent à ces décisions importantes de la Conférence générale :

- La création du Système métrique décimal au moment de la Révolution française et le dépôt qui en a résulté, le 22 juin 1799, de deux étalons en platine représentant le mètre et le kilogramme aux Archives de la République à Paris peuvent être considérés comme la première étape ayant conduit au Système international d'unités actuel.

- En 1832, Gauss œuvra activement en faveur de l'application du Système métrique, associé à la seconde, définie en astronomie, comme système cohérent d'unités pour les sciences physiques. Gauss fut le premier à faire des mesures absolues du champ magnétique terrestre en utilisant un système décimal fondé sur les trois unités mécaniques millimètre, gramme et seconde pour, respectivement, les grandeurs longueur, masse et temps. Par la suite, Gauss et Weber ont étendu ces mesures pour y inclure d'autres phénomènes électriques.

- Dans les années 1860, Maxwell et Thomson mirent en œuvre de manière plus complète ces mesures dans les domaines de l'électricité et du magnétisme au sein de la British Association for the Advancement of Science (BAAS). Ils exprimèrent les règles de formation d'un système cohérent d'unités composé d'unités de base et d'unités dérivées. En 1874 la BAAS introduisit le système $C G S$, un système d'unités tri-dimensionnel cohérent fondé sur les trois unités mécaniques centimètre, gramme et seconde, et utilisant des préfixes allant de micro à méga pour exprimer les sous-multiples et multiples décimaux. C'est en grande partie sur l'utilisation de ce système que se fonda, par la suite, le développement expérimental des sciences physiques.

- Compléter de façon cohérente le système CGS pour les domaines de l'électricité et du magnétisme conduisit à choisir des unités d'amplitude peu adaptée à la pratique. Le BAAS et le Congrès international d'électricité, qui précéda la Commission électrotechnique internationale (CEI), approuvèrent, dans les années 1880, un système mutuellement cohérent d'unités pratiques. Parmi celles-ci figuraient l'ohm pour la résistance électrique, le volt pour la force électromotrice et l'ampère pour le courant électrique.

- Après la signature de la Convention du Mètre le 20 mai 1875, qui créa le Bureau international des poids et mesures et établit le Comité international et la Conférence générale, on fabriqua de nouveaux prototypes internationaux du mètre et du kilogramme, approuvés en 1889 par la $1{ }^{\text {re }}$ CGPM. Avec la seconde des astronomes comme unité de temps, ces unités constituaient un système d'unités 
mécaniques tri-dimensionnel similaire au système CGS, mais dont les unités de base étaient le mètre, le kilogramme et la seconde (système MKS).

- En 1901, Giorgi montra qu'il était possible de combiner les unités mécaniques du système mètre-kilogramme-seconde au système pratique d'unités électriques pour former un seul système cohérent quadri-dimensionnel en ajoutant à ces trois unités de base une quatrième unité, de nature électrique, telle que l'ampère ou l'ohm, et en rationalisant les équations utilisées en électromagnétisme. La proposition de Giorgi ouvrit la voie à d'autres extensions.

- Après la révision de la Convention du Mètre par la $6^{e}$ CGPM en 1921, qui étendit les attributions et les responsabilités du Bureau international à d'autres domaines de la physique, et la création du Comité consultatif d'électricité (CCE) par la $7^{\mathrm{e}}$ CGPM qui en a résulté en 1927, la proposition de Giorgi fut discutée en détail par la CEI, l'Union internationale de physique pure et appliquée (UIPPA) et d'autres organisations internationales. Ces discussions conduisirent le CCE à proposer, en 1939, l'adoption d'un système quadri-dimensionnel fondé sur le mètre, le kilogramme, la seconde et l'ampère (système MKSA), une proposition qui fut approuvée par le Comité international en 1946.

- À la suite d'une enquête internationale effectuée par le Bureau international à partir de 1948, la 10 CGPM, en 1954, approuva l'introduction de l'ampère, du kelvin et de la candela comme unités de base, respectivement pour le courant électrique, la température thermodynamique et l'intensité lumineuse. La $11^{\mathrm{e}}$ CGPM donna le nom Système international d'unités $(S I)$ à ce système en 1960. Lors de la $14^{\mathrm{e}}$ CGPM, en 1971, après de longues discussions entre physiciens et chimistes pour trouver une définition capable de satisfaire les deux communautés, la mole fut ajoutée au SI comme unité de base pour la quantité de matière, portant à sept au total le nombre d'unités de base du SI tel que nous le connaissons aujourd'hui. 


\section{$2 \quad$ Unités SI}

\section{$2.1 \quad$ Unités SI de base}

Les définitions officielles de toutes les unités de base du SI sont approuvées par la Conférence générale. La première de ces définitions fut approuvée en 1889 et la plus récente en 1983. Ces définitions sont modifiées de temps à autre pour suivre l'évolution des sciences.

\subsubsection{Définitions}

Les définitions actuelles des unités de base, chacune extraite du volume des Comptes rendus de la Conférence générale (CR) qui l'a approuvée, apparaît ici en retrait et en caractères gras sans empattement. Les décisions de nature explicative qui ne font pas partie intégrante de la définition, extraites des Comptes rendus de la Conférence générale ou des Procès-verbaux du Comité international (PV), figurent aussi en retrait, mais en caractères sans empattement maigres. Le texte principal fournit des notes historiques et des explications, mais ne fait pas partie intégrante des définitions.

Il est important de faire la distinction entre la définition d'une unité et la réalisation de cette définition. La définition de chaque unité de base du SI est rédigée avec soin de manière à ce qu'elle soit unique et qu'elle fournisse un fondement théorique solide permettant d'effectuer les mesures les plus exactes et les plus reproductibles. La réalisation de la définition d'une unité est la procédure selon laquelle la définition de l'unité peut être utilisée afin d'établir la valeur et l'incertitude associée d'une grandeur de même nature que l'unité. Une description de la façon dont les définitions de certaines unités importantes sont réalisées en pratique figure sur le site Web du BIPM, à l'adresse : www.bipm.org/fr/si/si brochure/appendix2/.

Les unités SI dérivées cohérentes sont définies de manière unique et seulement en fonction des unités de base du SI. Par exemple, l'unité cohérente dérivée SI de résistance, l'ohm, symbole $\Omega$, est définie de manière unique par la relation $\Omega=\mathrm{m}^{2} \mathrm{~kg} \mathrm{~s}^{-3} \mathrm{~A}^{-2}$, qui résulte de la définition de la grandeur résistance. Cependant, il est possible d'utiliser n'importe quelle méthode en accord avec les lois de la physique pour réaliser n'importe laquelle des unités SI. En pratique, l'unité ohm peut être réalisée avec une exactitude élevée au moyen de l'effet Hall quantique et de la valeur de la constante de von Klitzing recommandée par le Comité international (voir pp. 74 et 77 de l'annexe 1).

Enfin, il faut reconnaître que même si les sept grandeurs de base - longueur, masse, temps, courant électrique, température thermodynamique, quantité de matière et intensité lumineuse - sont considérées comme indépendantes par convention, les unités de base - le mètre, le kilogramme, la seconde, l'ampère, le kelvin, la mole et la candela - ne le sont pas. Ainsi la définition du mètre fait appel à la seconde; la définition de l'ampère fait appel au mètre, au kilogramme et à la seconde; la définition de la mole fait appel au kilogramme; et la définition de la candela fait appel au mètre, au kilogramme et à la seconde. 


\subsubsection{Unité de longueur (mètre)}

La définition du mètre fondée sur le prototype international en platine iridié, en vigueur depuis 1889, avait été remplacée lors de la $11^{\text {e }}$ CGPM (1960) par une définition fondée sur la longueur d'onde d'une radiation du krypton 86, afin d'améliorer l'exactitude de la réalisation de la définition du mètre. Cette réalisation était effectuée au moyen d'un interféromètre et d'un microscope mobile en translation utilisés pour mesurer la variation des trajets optiques par comptage de franges. La $17^{\mathrm{e}}$ CGPM (1983, Résolution 1; CR, 97 et Metrologia, 1984, 20, 25) a remplacé en 1983 cette définition par la définition actuelle :

\section{Le mètre est la longueur du trajet parcouru dans le vide par la lumière pendant une durée de 1/299 792458 de seconde.}

Il en résulte que la vitesse de la lumière dans le vide est égale à 299792458 mètres par seconde exactement, $c_{0}=299792458 \mathrm{~m} / \mathrm{s}$.

Le prototype international du mètre originel, qui fut approuvé par la $1^{\text {re }}$ CGPM en 1889 (CR, 34-38), est toujours conservé au BIPM dans les conditions fixées en 1889.

\subsubsection{Unité de masse (kilogramme)}

Le prototype international du kilogramme, un objet fabriqué spécialement en platine iridié, est conservé au BIPM dans les conditions fixées par la $1^{\text {re }}$ CGPM en 1889 (CR, 34-38) lorsqu'elle approuva ce prototype et déclara :

Ce prototype sera considéré désormais comme unité de masse.

La $3^{\mathrm{e}}$ CGPM (1901; CR, 70), dans une déclaration tendant à faire cesser l'ambiguïté qui existait dans l'usage courant sur l'utilisation du terme " poids », confirma que :

\section{Le kilogramme est l'unité de masse ; il est égal à la masse du prototype international du kilogramme.}

La déclaration complète figure p. 53.

Il en résulte que la masse du prototype international du kilogramme est toujours égale à 1 kilogramme exactement, $m(\mathcal{K})=1 \mathrm{~kg}$. Cependant, en raison de l'accumulation inévitable de polluants sur les surfaces, le prototype international subit une contamination réversible de surface d'environ $1 \mu \mathrm{g}$ par an en masse. C'est pourquoi le Comité international a déclaré que, jusqu'à plus ample information, la masse de référence du prototype international est celle qui suit immédiatement le nettoyagelavage selon une méthode spécifique (PV, 1989, 57, 15-16 et PV, 1990, 58, 10-12). La masse de référence ainsi définie est utilisée pour étalonner les étalons nationaux en platine iridié (Metrologia, 1994, 31, 317-336).

\subsubsection{Unité de temps (seconde)}

La seconde, unité de temps, fut définie à l'origine comme la fraction 1/86 400 du jour solaire moyen. La définition exacte du « jour solaire moyen » était laissée aux astronomes. Toutefois, les observations ont montré que cette définition n'était pas satisfaisante par suite des irrégularités de la rotation de la Terre. Pour donner plus de précision à la définition de l'unité de temps, la $11^{\mathrm{e}}$ CGPM (1960, Résolution 9; CR, 86) approuva une définition, donnée par l'Union astronomique internationale, qui était fondée sur l'année tropique 1900. Cependant, les recherches expérimentales avaient déjà montré qu'un étalon atomique de temps, fondé sur une transition entre
Le symbole $c_{0}$ (ou parfois simplement $c$ ) est le symbole conventionnel pour la vitesse de la lumière dans le vide.
Le symbole $m(\mathcal{K})$ est utilisé pour désigner la masse du prototype international du kilogramme, $K$. 
deux niveaux d'énergie d'un atome ou d'une molécule, pourrait être réalisé et reproduit avec une exactitude beaucoup plus élevée. Considérant qu'une définition de haute précision de l'unité de temps du Système international était indispensable pour la science et la technologie, la $13^{\mathrm{e}}$ CGPM (1967/68, Résolution 1; CR, 103 et Metrologia, 1968, 4, 43) a remplacé la définition de la seconde par la suivante :

La seconde est la durée de 9192631770 périodes de la radiation correspondant à la transition entre les deux niveaux hyperfins de l'état fondamental de l'atome de césium 133.

Il en résulte que la fréquence de la transition hyperfine de l'état fondamental de l'atome de césium est égale à 9192631770 hertz exactement, $v(\mathrm{hfs} \mathrm{Cs})=9192631770 \mathrm{~Hz}$. Lors de sa session de 1997, le Comité international a confirmé que :

Cette définition se réfère à un atome de césium au repos, à une température de $0 \mathrm{~K}$.

Cette note a pour objet de préciser que la définition de la seconde du SI est fondée sur un atome de césium non perturbé par le rayonnement du corps noir, c'est-à-dire dans un environnement maintenu à une température thermodynamique de $0 \mathrm{~K}$. Les fréquences de tous les étalons primaires de fréquence doivent donc être corrigées pour tenir compte du décalage dû au rayonnement ambiant, comme l'a précisé le Comité consultatif du temps et des fréquences en 1999.

\subsubsection{Unité de courant électrique (ampère)}

Des unités électriques, dites « internationales », pour le courant et pour la résistance, avaient été introduites par le Congrès international d'électricité, tenu à Chicago en 1893 , et les définitions de l'ampère «international » et de l'ohm «international » furent confirmées par la Conférence internationale de Londres en 1908.

Bien qu'une opinion unanime de remplacer ces unités « internationales » par des unités dites «absolues » fût déjà exprimée à l'occasion de la $8^{e}$ CGPM (1933), la décision officielle de supprimer ces unités « internationales» ne fut prise que par la $9^{\mathrm{e}}$ CGPM (1948) qui adopta l'ampère comme unité de courant électrique, selon la définition suivante proposée par le Comité international (1946, Résolution 2 ; PV, 20, 129-137) :

L'ampère est l'intensité d'un courant constant qui, maintenu dans deux conducteurs parallèles, rectilignes, de longueur infinie, de section circulaire négligeable et placés à une distance de 1 mètre l'un de l'autre dans le vide, produirait entre ces conducteurs une force égale à $2 \times 10^{-7}$ newton par mètre de longueur.

Il en résulte que la constante magnétique, aussi connue sous le nom de perméabilité $\mathrm{du}$ vide, est égale à $4 \pi \times 10^{-7}$ henrys par mètre exactement, $\mu_{0}=4 \pi \times 10^{-7} \mathrm{H} / \mathrm{m}$.

L'expression « unité MKS de force » qui figure dans le texte original de 1946 a été remplacée ici par «newton», nom adopté pour cette unité par la 9e CGPM (1948, Résolution 7 ; CR, 70).

\subsubsection{Unité de température thermodynamique (kelvin)}

La définition de l'unité de température thermodynamique fut en fait donnée par la $10^{\mathrm{e}}$ CGPM (1954, Résolution 3; CR, 79) qui choisit le point triple de l'eau comme point fixe fondamental en lui attribuant la température de $273,16 \mathrm{~K}$ par définition. $\mathrm{La}$ 
$13^{\mathrm{e}}$ CGPM (1967/68, Résolution 3 ; CR, 104 et Metrologia, 1968, 4, 43) adopta le nom kelvin, symbole $\mathrm{K}$, au lieu de « degré Kelvin », symbole ${ }^{\circ} \mathrm{K}$, et définit l'unité de température thermodynamique comme suit (1967/68, Résolution 4; CR, 104 et Metrologia, 1968, 4, 43) :

\section{Le kelvin, unité de température thermodynamique, est la fraction 1/273,16 de la température thermodynamique du point triple de l'eau.}

Il en résulte que la température thermodynamique du point triple de l'eau est égale à 273,16 kelvins exactement, $T_{\text {tpw }}=273,16 \mathrm{~K}$.

Lors de sa session de 2005 le Comité international a affirmé que :

Cette définition se réfère à une eau d'une composition isotopique définie par les rapports de quantité de matière suivants : 0,00015576 mole de ${ }^{2} \mathrm{H}$ par mole de ${ }^{1} \mathrm{H}, 0,0003799$ mole de ${ }^{17} \mathrm{O}$ par mole de ${ }^{16} \mathrm{O}$ et 0,0020052 mole de ${ }^{18} \mathrm{O}$ par mole de ${ }^{16} \mathrm{O}$.

En raison de la manière dont les échelles de température étaient habituellement définies, il resta d'usage courant d'exprimer la température thermodynamique, symbole $T$, en fonction de sa différence par rapport à la température de référence $T_{0}=273,15 \mathrm{~K}$, le point de congélation de l'eau. Cette différence de température est appelée température Celsius, symbole $t$, et elle est définie par l'équation entre grandeurs :

$$
t=T-T_{0} .
$$

L'unité de température Celsius est le degré Celsius, symbole ${ }^{\circ} \mathrm{C}$, égal à l'unité kelvin par définition. Une différence ou un intervalle de température peut s'exprimer aussi bien en kelvins qu'en degrés Celsius (13 ${ }^{\mathrm{e}}$ CGPM, 1967/68, Résolution 3, mentionnée ci-dessus), la valeur numérique de la différence de température étant la même. Cependant, la valeur numérique de la température Celsius exprimée en degrés Celsius est liée à la valeur numérique de la température thermodynamique exprimée en kelvins par la relation :

$$
t /{ }^{\circ} \mathrm{C}=T / \mathrm{K}-273,15 .
$$

Le kelvin et le degré Celsius sont aussi les unités de l'Échelle internationale de température de 1990 (EIT-90) adoptée par le Comité international en 1989 dans sa Recommandation 5 (CI-1989 ; PV, 57, 26 et Metrologia, 1990, 27, 13).

\subsubsection{Unité de quantité de matière (mole)}

Après la découverte des lois fondamentales de la chimie, on a utilisé, pour spécifier les quantités des divers éléments et composés chimiques, des unités portant par exemple les noms de « atome-gramme » et «molécule-gramme ». Ces unités étaient liées directement aux « poids atomiques » et aux « poids moléculaires » qui étaient en réalité des masses relatives. Les "poids atomiques» furent d'abord rapportés à celui de l'élément chimique oxygène, pris par convention égal à 16. Mais, tandis que les physiciens séparaient les isotopes au spectromètre de masse et attribuaient la valeur 16 à l'un des isotopes de l'oxygène, les chimistes attribuaient la même valeur au mélange (de composition légèrement variable) des isotopes 16,17 et 18 qui constitue l'élément oxygène naturel. Un accord entre l'Union internationale de physique pure et appliquée (UIPPA) et l'Union internationale de chimie pure et appliquée (UICPA) mit fin à cette dualité en 1959/60. Depuis lors, physiciens et chimistes sont convenus d'attribuer la valeur 12, exactement, au « poids atomique » de l'isotope 12 du carbone (carbone $12,{ }^{12} \mathrm{C}$ ), ou selon une formulation plus correcte à la masse atomique
Le symbole $T_{\text {tpw }}$ est utilisé pour désigner la température thermodynamique du point triple de l'eau. 
relative $\left.A_{\mathrm{r}}{ }^{12} \mathrm{C}\right)$. L'échelle unifiée ainsi obtenue donne les valeurs des masses atomiques et moléculaires relatives, aussi connues sous le nom de poids atomiques et moléculaires, respectivement.

La grandeur utilisée par les chimistes pour spécifier la quantité d'éléments ou de composés chimiques est maintenant appelée «quantité de matière ». La quantité de matière est définie comme étant proportionnelle au nombre d'entités élémentaires d'un échantillon, la constante de proportionnalité étant une constante universelle identique pour tous les échantillons. L'unité de quantité de matière est appelée la mole, symbole mol, et la mole est définie en fixant la masse de carbone 12 qui constitue une mole d'atomes de carbone 12. Par un accord international, cette masse a été fixée à $0,012 \mathrm{~kg}$, c'est-à-dire $12 \mathrm{~g}$.

Suivant les propositions de l'UIPPA, de l'UICPA et de l'ISO, le Comité international donna en 1967 et confirma en 1969 une définition de la mole qui fut finalement adoptée par la $14^{\mathrm{e}}$ CGPM (1971, Résolution 3 ; CR, 78 et Metrologia, 1972, 8, 36) :

1. La mole est la quantité de matière d'un système contenant autant d'entités élémentaires qu'il y a d'atomes dans 0,012 kilogramme de carbone 12 ; son symbole est « $\mathrm{mol}$ ».

2. Lorsqu'on emploie la mole, les entités élémentaires doivent être spécifiées et peuvent être des atomes, des molécules, des ions, des électrons, d'autres particules ou des groupements spécifiés de telles particules.

Il en résulte que la masse molaire du carbone 12 est égale à 0,012 kilogramme par mole exactement, $M\left({ }^{12} \mathrm{C}\right)=12 \mathrm{~g} / \mathrm{mol}$.

En 1980, le Comité international a approuvé le rapport du CCU (1980) qui précisait :

Dans cette définition, il est entendu que l'on se réfère à des atomes de carbone 12 non liés, au repos et dans leur état fondamental.

La définition de la mole permet aussi de déterminer la valeur de la constante universelle qui relie le nombre d'entités à la quantité de matière d'un échantillon. Cette constante est appelée constante d'Avogadro, symbole $N_{\mathrm{A}}$ ou $L$. Si $N(\mathrm{X})$ désigne le nombre d'entités $\mathrm{X}$ d'un échantillon donné, et si $n(\mathrm{X})$ désigne la quantité de matière d'entités $\mathrm{X}$ du même échantillon, on obtient la relation :

$$
n(\mathrm{X})=N(\mathrm{X}) / N_{\mathrm{A}} \text {. }
$$

Notons que puisque $N(\mathrm{X})$ est sans dimension, et puisque $n(\mathrm{X})$ est exprimé par l'unité SI mole, la constante d'Avogadro a pour unité SI la mole à la puissance moins un.

Dans le nom «quantité de matière », les mots «de matière» pourraient être simplement remplacés par d'autres mots précisant la matière en question pour chaque application particulière, ainsi par exemple on pourrait parler de « quantité de chlorure d'hydrogène, $\mathrm{HCl}$ » ou de « quantité de benzène, $\mathrm{C}_{6} \mathrm{H}_{6}$ 》. Il est important de toujours préciser l'entité en question (comme le souligne la seconde phrase de la définition de la mole), de préférence en donnant la formule chimique empirique du matériau concerné. Bien que le mot "quantité» ait une définition plus générale dans le dictionnaire, cette abréviation du nom complet «quantité de matière » est parfois utilisée par souci de concision. Ceci s'applique aussi aux grandeurs dérivées telles que la «concentration de quantité de matière », qui peut simplement être appelée « concentration de quantité ». Toutefois, dans le domaine de la chimie clinique, le nom «concentration de quantité de matière» est généralement abrégé en « concentration de matière ».
Le symbole recommandé pour la masse atomique relative (poids atomique) est $A_{\mathrm{r}}(\mathrm{X})$, dont il faut préciser l'entité atomique $\mathrm{X}$, et le symbole recommandé pour la masse moléculaire relative (poids moléculaire) est $M_{\mathrm{r}}(\mathrm{X})$, dont il faut préciser l'entité moléculaire $\mathrm{X}$.

La masse molaire d'un atome ou d'une molécule $\mathrm{X}$ est désignée par $M(\mathrm{X})$ ou $M_{\mathrm{X}}$; c'est la masse d'une mole de X.

Lorsque l'on cite la définition de la mole, il convient de lui adjoindre aussi cette remarque. 


\subsubsection{Unité d'intensité lumineuse (candela)}

Les unités d'intensité lumineuse fondées sur des étalons à flamme ou à filament incandescent, qui étaient en usage dans différents pays avant 1948, furent d'abord remplacées par la « bougie nouvelle» fondée sur la luminance du radiateur de Planck (corps noir) à la température de congélation du platine. Cette modification avait été préparée dès avant 1937 par la Commission internationale de l'éclairage (CIE) et par le Comité international ; la décision fut prise par le Comité international en 1946. Elle fut ratifiée en 1948 par la $9^{\mathrm{e}}$ CGPM qui adopta pour cette unité un nouveau nom international, la candela, symbole cd ; en 1967, la 13 ${ }^{\mathrm{e}}$ CGPM (Résolution 5 ; CR, 104 et Metrologia, 1968, 4, 43-44) donna une forme modifiée à la définition de 1946.

En 1979, en raison des difficultés expérimentales liées à la réalisation du radiateur de Planck aux températures élevées et des possibilités nouvelles offertes par la radiométrie, c'est-à-dire la mesure de la puissance des rayonnements optiques, la $16^{\mathrm{e}}$ CGPM (1979, Résolution 3 ; CR, 100 et Metrologia, 1980, 16, 56) adopta une nouvelle définition de la candela :

La candela est l'intensité lumineuse, dans une direction donnée, d'une source qui émet un rayonnement monochromatique de fréquence $540 \times 10^{12}$ hertz et dont l'intensité énergétique dans cette direction est $1 / 683$ watt par stéradian.

Il en résulte que l'efficacité lumineuse spectrale d'un rayonnement monochromatique de fréquence $540 \times 10^{12}$ hertz est égale à 683 lumens par watt exactement, $K=683 \mathrm{~lm} / \mathrm{W}=683 \mathrm{~cd} \mathrm{sr} / \mathrm{W}$.

\subsubsection{Symboles des sept unités de base}

Les unités de base du Système international sont rassemblées dans le tableau 1 qui relie les grandeurs de base aux noms et symboles des sept unités de base $\left(10^{\mathrm{e}} \mathrm{CGPM}\right.$ (1954, Résolution 6; CR, 80); $11^{\mathrm{e}}$ CGPM (1960, Résolution 12; CR, 87); $13^{\mathrm{e}}$ CGPM (1967/68, Résolution 3 ; CR, 104 et Metrologia, 1968, 4, 43); $14^{\mathrm{e}}$ CGPM (1971, Résolution 3 ; CR, 78 et Metrologia, 1972, 8, 36)).

Tableau 1. Unités de base du SI

\begin{tabular}{lllll}
\hline \multirow{2}{*}{ Grandeur de base } & & \multicolumn{2}{l}{ Unité SI de base } \\
Nom & Symbole & & Nom & Symbole \\
\hline longueur & $l, x, r$, etc. & & mètre & $\mathrm{m}$ \\
masse & $m$ & & kilogramme & $\mathrm{kg}$ \\
temps, durée & $t$ & & seconde & $\mathrm{s}$ \\
courant électrique & $I, i$ & & ampère & $\mathrm{A}$ \\
température thermodynamique & $T$ & kelvin & $\mathrm{K}$ \\
quantité de matière & $n$ & mole & mol \\
intensité lumineuse & $I_{\mathrm{v}}$ & candela & $\mathrm{cd}$ \\
\hline
\end{tabular}

Les symboles indiqués pour les grandeurs sont généralement de simples lettres de l'alphabet grec ou latin, en italique, et constituent des recommandations. L'utilisation des symboles indiqués pour les unités est obligatoire, voir chapitre 5 . 


\section{$2.2 \quad$ Unités SI dérivées}

Les unités dérivées sont formées à partir de produits de puissances des unités de base. Les unités dérivées cohérentes sont des produits de puissances des unités de base qui ne font pas intervenir d'autre facteur numérique que 1 . Les unités de base et les unités dérivées cohérentes du SI forment un ensemble cohérent, désigné sous le nom d'ensemble d'unités SI cohérentes (voir 1.4, p. 16).

\subsubsection{Unités dérivées exprimées à partir des unités de base}

Les grandeurs utilisées dans le domaine scientifique sont en nombre illimité ; il n'est donc pas possible de fournir une liste complète des grandeurs et unités dérivées. Le tableau 2 présente un certain nombre d'exemples de grandeurs dérivées, avec les unités dérivées cohérentes correspondantes exprimées directement en fonction des unités de base.

Tableau 2. Exemples d'unités SI dérivées cohérentes exprimées à partir des unités de base

\begin{tabular}{|c|c|c|c|}
\hline \multicolumn{2}{|l|}{ Grandeur dérivée } & \multicolumn{2}{|l|}{ Unité SI dérivée cohérente } \\
\hline Nom & Symbole & Nom & Symbole \\
\hline superficie & $A$ & mètre carré & $\mathrm{m}^{2}$ \\
\hline volume & $V$ & mètre cube & $\mathrm{m}^{3}$ \\
\hline vitesse & $v$ & mètre par seconde & $\mathrm{m} / \mathrm{s}$ \\
\hline accélération & $a$ & mètre par seconde carrée & $\mathrm{m} / \mathrm{s}^{2}$ \\
\hline nombre d'ondes & $\sigma, \widetilde{v}$ & mètre à la puissance moins un & $\mathrm{m}^{-1}$ \\
\hline masse volumique & $\rho$ & kilogramme par mètre cube & $\mathrm{kg} / \mathrm{m}^{3}$ \\
\hline masse surfacique & $\rho_{\mathrm{A}}$ & kilogramme par mètre carré & $\mathrm{kg} / \mathrm{m}^{2}$ \\
\hline volume massique & $v$ & mètre cube par kilogramme & $\mathrm{m}^{3} / \mathrm{kg}$ \\
\hline densité de courant & $j$ & ampère par mètre carré & $\mathrm{A} / \mathrm{m}^{2}$ \\
\hline champ magnétique & $H$ & ampère par mètre & $\mathrm{A} / \mathrm{m}$ \\
\hline concentration & $c$ & mole par mètre cube & $\mathrm{mol} / \mathrm{m}^{3}$ \\
\hline $\begin{array}{l}\text { de quantité de matière }{ }^{(a)} \text {, } \\
\text { concentration }\end{array}$ & & & \\
\hline concentration massique & $\rho, \gamma$ & kilogramme par mètre cube & $\mathrm{kg} / \mathrm{m}^{3}$ \\
\hline luminance lumineuse & $L_{\mathrm{V}}$ & candela par mètre carré & $\mathrm{cd} / \mathrm{m}^{2}$ \\
\hline indice de réfraction ${ }^{(b)}$ & $n$ & un & 1 \\
\hline perméabilité relative $^{(b)}$ & $\mu_{\mathrm{r}}$ & un & 1 \\
\hline
\end{tabular}

(a) Dans le domaine de la chimie clinique, cette grandeur est aussi appelée concentration de matière.

(b) Ce sont des grandeurs sans dimension, ou grandeurs de dimension un. Le symbole « $1 »$ pour l'unité (le nombre « un ») n'est généralement pas mentionné lorsque l'on précise la valeur des grandeurs sans dimension.

\subsubsection{Unités ayant des noms spéciaux et des symboles particuliers ; unités faisant appel à des noms spéciaux et des symboles particuliers}

Par souci de commodité, certaines unités dérivées cohérentes ont reçu un nom spécial et un symbole particulier. Elles sont au nombre de vingt-deux et sont mentionnées au tableau 3. Ces noms spéciaux et ces symboles particuliers peuvent eux-mêmes être utilisés avec les noms et les symboles d'autres unités de base ou dérivées pour exprimer les unités d'autres grandeurs dérivées. Quelques exemples figurent au tableau 4. Les noms spéciaux et les symboles particuliers permettent d'exprimer, sous 
une forme condensée, des combinaisons d'unités de base fréquemment utilisées, mais souvent ils servent aussi à rappeler au lecteur la nature de la grandeur concernée. Les préfixes SI peuvent être utilisés avec n'importe quel nom spécial et symbole particulier, mais dans ce cas l'unité qui en résulte n'est plus une unité cohérente.

Les quatre derniers noms et symboles d'unités figurant au bas du tableau 3 sont particuliers : ils furent spécifiquement approuvés par la $15^{\mathrm{e}}$ CGPM (1975, Résolutions 8 et 9; CR, 105 et Metrologia, 1975, 11, 180), la 16 CGPM (1979, Résolution 5 ; CR, 100 et Metrologia, 1980, 16, 56) et la $21^{\mathrm{e}} \mathrm{CGPM}(1999$, Résolution 12 ; CR, 145 et Metrologia, 2000, 37, 95) pour éviter des erreurs dans les mesures liées à la santé humaine.

Dans la dernière colonne des tableaux 3 et 4 , on trouve l'expression des unités SI mentionnées en fonction des unités SI de base. Dans cette colonne, les facteurs tels que $\mathrm{m}^{0}, \mathrm{~kg}^{0}$, etc., considérés comme égaux à 1 , ne sont pas écrits explicitement.

Tableau 3. Unités SI dérivées cohérentes ayant des noms spéciaux et des symboles particuliers

\begin{tabular}{|c|c|c|c|c|}
\hline \multirow[b]{2}{*}{ Grandeur dérivée } & \multicolumn{4}{|c|}{ Unité SI dérivée cohérente ${ }^{(a)}$} \\
\hline & Nom & Symbole & $\begin{array}{l}\text { Expression } \\
\text { utilisant } \\
\text { d'autres } \\
\text { unités SI }\end{array}$ & $\begin{array}{l}\text { Expression } \\
\text { en unités SI } \\
\text { de base }\end{array}$ \\
\hline angle plan & $\operatorname{radian}^{(b)}$ & $\mathrm{rad}$ & $1^{(b)}$ & $\mathrm{m} / \mathrm{m}$ \\
\hline angle solide & stéradian $^{(b)}$ & $\mathrm{sr}^{(c)}$ & $1^{(b)}$ & $\mathrm{m}^{2} / \mathrm{m}^{2}$ \\
\hline fréquence & hertz $^{(d)}$ & $\mathrm{Hz}$ & & $\mathrm{s}^{-1}$ \\
\hline force & newton & $\mathrm{N}$ & & $\mathrm{m} \mathrm{kg} \mathrm{s}^{-2}$ \\
\hline pression, contrainte & pascal & $\mathrm{Pa}$ & $\mathrm{N} / \mathrm{m}^{2}$ & $\mathrm{~m}^{-1} \mathrm{~kg} \mathrm{~s}^{-2}$ \\
\hline $\begin{array}{l}\text { énergie, travail, } \\
\text { quantité de chaleur }\end{array}$ & joule & $\mathrm{J}$ & $\mathrm{N} \mathrm{m}$ & $\mathrm{m}^{2} \mathrm{~kg} \mathrm{~s}^{-2}$ \\
\hline puissance, flux énergétique & watt & $\mathrm{W}$ & $\mathrm{J} / \mathrm{s}$ & $\mathrm{m}^{2} \mathrm{~kg} \mathrm{~s}^{-3}$ \\
\hline $\begin{array}{l}\text { charge électrique, } \\
\text { quantité d'électricité }\end{array}$ & coulomb & $\mathrm{C}$ & & $\mathrm{s} \mathrm{A}$ \\
\hline $\begin{array}{l}\text { différence de potentiel électrique } \\
\text { force électromotrice }\end{array}$ & volt & $\mathrm{V}$ & W/A & $\mathrm{m}^{2} \mathrm{~kg} \mathrm{~s}^{-3} \mathrm{~A}^{-1}$ \\
\hline capacité électrique & farad & $\mathrm{F}$ & $\mathrm{C} / \mathrm{V}$ & $\mathrm{m}^{-2} \mathrm{~kg}^{-1} \mathrm{~s}^{4} \mathrm{~A}^{2}$ \\
\hline résistance électrique & ohm & $\Omega$ & $\mathrm{V} / \mathrm{A}$ & $\mathrm{m}^{2} \mathrm{~kg} \mathrm{~s}^{-3} \mathrm{~A}^{-2}$ \\
\hline conductance électrique & siemens & $\mathrm{S}$ & $\mathrm{A} / \mathrm{V}$ & $m^{-2} \mathrm{~kg}^{-1} \mathrm{~s}^{3} \mathrm{~A}^{2}$ \\
\hline flux d'induction magnétique & weber & $\mathrm{Wb}$ & V s & $\mathrm{m}^{2} \mathrm{~kg} \mathrm{~s}^{-2} \mathrm{~A}^{-1}$ \\
\hline induction magnétique & tesla & $\mathrm{T}$ & $\mathrm{Wb} / \mathrm{m}^{2}$ & $\mathrm{~kg} \mathrm{~s}^{-2} \mathrm{~A}^{-1}$ \\
\hline inductance & henry & $\mathrm{H}$ & $\mathrm{Wb} / \mathrm{A}$ & $\mathrm{m}^{2} \mathrm{~kg} \mathrm{~s}^{-2} \mathrm{~A}^{-2}$ \\
\hline température Celsius & degré Celsius ${ }^{(e)}$ & ${ }^{\circ} \mathrm{C}$ & & $\mathrm{K}$ \\
\hline flux lumineux & lumen & $\operatorname{lm}$ & $\mathrm{cd} \mathrm{sr}^{(c)}$ & $\mathrm{cd}$ \\
\hline éclairement lumineux & $\operatorname{lux}$ & lx & $1 \mathrm{~m} / \mathrm{m}^{2}$ & $\mathrm{~m}^{-2} \mathrm{~cd}$ \\
\hline activité d'un radionucléide $(f)$ & becquerel $^{(d)}$ & $\mathrm{Bq}$ & & $\mathrm{s}^{-1}$ \\
\hline $\begin{array}{l}\text { dose absorbée, } \\
\text { énergie massique } \\
\text { (communiquée), kerma }\end{array}$ & gray & Gy & $\mathrm{J} / \mathrm{kg}$ & $\mathrm{m}^{2} \mathrm{~s}^{-2}$ \\
\hline $\begin{array}{l}\text { équivalent de dose, } \\
\text { équivalent de dose ambiant, } \\
\text { équivalent de dose directionnel, } \\
\text { équivalent de dose individuel, } \\
\text { activité catalytique }\end{array}$ & $\begin{array}{l}\text { sievert }^{(g)} \\
\text { katal }\end{array}$ & Sv & $\mathrm{J} / \mathrm{kg}$ & $\mathrm{s}^{-1} \mathrm{~mol}$ \\
\hline
\end{tabular}


(a) Les préfixes SI peuvent être utilisés avec n'importe quel nom spécial et symbole particulier, mais dans ce cas l'unité qui en résulte n'est plus une unité cohérente.

(b) Le radian et le stéradian sont des noms spéciaux pour le nombre un, qui peuvent être utilisés pour donner des informations sur la grandeur concernée. En pratique, les symboles rad et sr sont utilisés lorsque c'est utile, et le symbole pour l'unité dérivée « un » n'est généralement pas mentionné lorsque l'on donne les valeurs des grandeurs sans dimension.

(c) En photométrie, on maintient généralement le nom et le symbole du stéradian, sr, dans l'expression des unités.

(d) Le hertz est uniquement utilisé pour les phénomènes périodiques, et le becquerel pour les processus aléatoires liés à la mesure de l'activité d'un radionucléide.

(e) Le degré Celsius est le nom spécial du kelvin utilisé pour exprimer les températures Celsius. Le degré Celsius et le kelvin ont la même taille, ainsi la valeur numérique d'une différence de température ou d'un intervalle de température est identique quand elle est exprimée en degrés Celsius ou en kelvins.

(f) L'activité d'un radionucléide est parfois appelée de manière incorrecte radioactivité.

(g) Voir la Recommandation 2 (CI-2002) du CIPM (p. 79) sur l'utilisation du sievert (PV, 2002, 70, 102).

Tableau 4. Exemples d'unités SI dérivées cohérentes dont le nom et le symbole comprennent des unités SI dérivées cohérentes ayant des noms spéciauX et des symboles particuliers

Unité SI dérivée cohérente

\begin{tabular}{|c|c|c|c|}
\hline \multirow[b]{2}{*}{ Grandeur dérivée } & \\
\hline & Nom & Symbole & $\begin{array}{l}\text { Expression } \\
\text { en unités SI de base }\end{array}$ \\
\hline viscosité dynamique & pascal seconde & $\mathrm{Pas}$ & $\mathrm{m}^{-1} \mathrm{~kg} \mathrm{~s}^{-1}$ \\
\hline moment d'une force & newton mètre & $\mathrm{N} \mathrm{m}$ & $\mathrm{m}^{2} \mathrm{~kg} \mathrm{~s}^{-2}$ \\
\hline tension superficielle & newton par mètre & $\mathrm{N} / \mathrm{m}$ & $\mathrm{kg} \mathrm{s}^{-2}$ \\
\hline vitesse angulaire & radian par seconde & $\mathrm{rad} / \mathrm{s}$ & $\mathrm{m} \mathrm{m}^{-1} \mathrm{~s}^{-1}=\mathrm{s}^{-1}$ \\
\hline accélération angulaire & radian par seconde carrée & $\mathrm{rad} / \mathrm{s}^{2}$ & $\mathrm{~m} \mathrm{~m}^{-1} \mathrm{~s}^{-2}=\mathrm{s}^{-2}$ \\
\hline $\begin{array}{l}\text { flux thermique surfacique, } \\
\text { éclairement énergétique }\end{array}$ & watt par mètre carré & $\mathrm{W} / \mathrm{m}^{2}$ & $\mathrm{~kg} \mathrm{~s}^{-3}$ \\
\hline capacité thermique, entropie & joule par kelvin & $\mathrm{J} / \mathrm{K}$ & $\mathrm{m}^{2} \mathrm{~kg} \mathrm{~s}^{-2} \mathrm{~K}^{-1}$ \\
\hline $\begin{array}{l}\text { capacité thermique massique, } \\
\text { entropie massique }\end{array}$ & joule par kilogramme kelvin & $\mathrm{J} /(\operatorname{kg~K})$ & $\mathrm{m}^{2} \mathrm{~s}^{-2} \mathrm{~K}^{-1}$ \\
\hline énergie massique & joule par kilogramme & $\mathrm{J} / \mathrm{kg}$ & $\mathrm{m}^{2} \mathrm{~s}^{-2}$ \\
\hline conductivité thermique & watt par mètre kelvin & $\mathrm{W} /(\mathrm{m} \mathrm{K})$ & $\mathrm{m} \mathrm{kg} \mathrm{s}^{-3} \mathrm{~K}^{-1}$ \\
\hline énergie volumique & joule par mètre cube & $\mathrm{J} / \mathrm{m}^{3}$ & $\mathrm{~m}^{-1} \mathrm{~kg} \mathrm{~s}^{-2}$ \\
\hline champ électrique & volt par mètre & $\mathrm{V} / \mathrm{m}$ & $\mathrm{m} \mathrm{kg} \mathrm{s}^{-3} \mathrm{~A}^{-1}$ \\
\hline charge électrique volumique & coulomb par mètre cube & $\mathrm{C} / \mathrm{m}^{3}$ & $\mathrm{~m}^{-3} \mathrm{~s} \mathrm{~A}$ \\
\hline charge électrique surfacique & coulomb par mètre carré & $\mathrm{C} / \mathrm{m}^{2}$ & $\mathrm{~m}^{-2} \mathrm{~s} \mathrm{~A}$ \\
\hline $\begin{array}{l}\text { induction électrique, } \\
\text { déplacement électrique }\end{array}$ & coulomb par mètre carré & $\mathrm{C} / \mathrm{m}^{2}$ & $\mathrm{~m}^{-2} \mathrm{~s} \mathrm{~A}$ \\
\hline permittivité & farad par mètre & $\mathrm{F} / \mathrm{m}$ & $\mathrm{m}^{-3} \mathrm{~kg}^{-1} \mathrm{~s}^{4} \mathrm{~A}^{2}$ \\
\hline perméabilité & henry par mètre & $\mathrm{H} / \mathrm{m}$ & $\mathrm{m} \mathrm{kg} \mathrm{s}^{-2} \mathrm{~A}^{-2}$ \\
\hline énergie molaire & joule par mole & $\mathrm{J} / \mathrm{mol}$ & $\mathrm{m}^{2} \mathrm{~kg} \mathrm{~s}^{-2} \mathrm{~mol}^{-1}$ \\
\hline $\begin{array}{l}\text { entropie molaire, } \\
\text { capacité thermique molaire }\end{array}$ & joule par mole kelvin & $\mathrm{J} /(\mathrm{mol} \mathrm{K})$ & $\mathrm{m}^{2} \mathrm{~kg} \mathrm{~s}^{-2} \mathrm{~K}^{-1} \mathrm{~mol}^{-1}$ \\
\hline exposition (rayons $x$ et $\gamma$ ) & coulomb par kilogramme & $\mathrm{C} / \mathrm{kg}$ & $\mathrm{kg}^{-1} \mathrm{~s} \mathrm{~A}$ \\
\hline débit de dose absorbée & gray par seconde & $\mathrm{Gy} / \mathrm{s}$ & $\mathrm{m}^{2} \mathrm{~s}^{-3}$ \\
\hline intensité énergétique & watt par stéradian & $\mathrm{W} / \mathrm{sr}$ & $\mathrm{m}^{4} \mathrm{~m}^{-2} \mathrm{~kg} \mathrm{~s}^{-3}=\mathrm{m}^{2} \mathrm{~kg} \mathrm{~s}^{-3}$ \\
\hline luminance énergétique & watt par mètre carré stéradian & $\mathrm{W} /\left(\mathrm{m}^{2} \mathrm{sr}\right)$ & $\mathrm{m}^{2} \mathrm{~m}^{-2} \mathrm{~kg} \mathrm{~s}^{-3}=\mathrm{kg} \mathrm{s}^{-3}$ \\
\hline $\begin{array}{l}\text { concentration de l'activité } \\
\text { catalytique }\end{array}$ & katal par mètre cube & $\mathrm{kat} / \mathrm{m}^{3}$ & $\mathrm{~m}^{-3} \mathrm{~s}^{-1} \mathrm{~mol}$ \\
\hline
\end{tabular}


Les valeurs de plusieurs grandeurs différentes peuvent être exprimées au moyen du même nom et symbole d'unité SI. Ainsi le joule par kelvin est le nom de l'unité SI pour la grandeur capacité thermique aussi bien que pour la grandeur entropie. De même, l'ampère est le nom de l'unité SI pour la grandeur de base courant électrique aussi bien que pour la grandeur dérivée force magnétomotrice. Il ne suffit donc pas, et c'est important, d'indiquer le nom de l'unité pour spécifier la grandeur mesurée. Cette règle s'applique non seulement aux textes scientifiques et techniques mais aussi, par exemple, aux appareils de mesure (c'est-à-dire qu'ils doivent porter non seulement l'indication de l'unité mais aussi l'indication de la grandeur mesurée).

Une unité dérivée peut souvent s'exprimer de plusieurs façons en utilisant des unités de base et des unités dérivées ayant des noms spéciaux : le joule, par exemple, peut s'écrire newton mètre, ou bien kilogramme mètre carré par seconde carrée. Cette liberté algébrique est toutefois limitée par des considérations physiques de bon sens, et selon les circonstances certaines formes peuvent être plus utiles que d'autres.

En pratique, afin de réduire le risque de confusion entre des grandeurs différentes ayant la même dimension, on exprime leur unité en employant de préférence un nom spécial ou une combinaison particulière de noms d'unités, rappelant comment la grandeur est définie. Par exemple, la grandeur « couple » peut être considérée comme résultant du produit vectoriel d'une force par une distance, ce qui suggère d'utiliser l'unité newton mètre, ou comme représentant une énergie par unité d'angle, ce qui suggère d'utiliser l'unité joule par radian. On appelle l'unité SI de fréquence hertz (un cycle par seconde), l'unité SI de vitesse angulaire radian par seconde et l'unité SI d'activité becquerel (une occurrence par seconde). Même s'il est correct d'écrire ces trois unités seconde à la puissance moins un, l'emploi de noms différents sert à souligner la différence de nature des grandeurs en question. Le fait d'utiliser l'unité radian par seconde pour exprimer la vitesse angulaire, et le hertz pour la fréquence, indique aussi que la valeur numérique de la fréquence en hertz doit être multipliée par $2 \pi$ pour obtenir la valeur numérique de la vitesse angulaire correspondante en radian par seconde.

Dans le domaine des rayonnements ionisants, on appelle l'unité SI d'activité le becquerel plutôt que seconde à la puissance moins un, et les unités SI de dose absorbée et d'équivalent de dose, respectivement, gray et sievert, plutôt que joule par kilogramme. Les noms spéciaux becquerel, gray et sievert ont été introduits spécialement en raison des dangers pour la santé humaine qui pourraient résulter d'erreurs dans le cas où les unités seconde à la puissance moins un et joule par kilogramme seraient à tort utilisées pour identifier les grandeurs.

\subsubsection{Unités des grandeurs sans dimension, aussi désignées grandeurs de dimension un}

Certaines grandeurs sont définies par le rapport de deux grandeurs de même nature ; elles sont donc sans dimension, ou leur dimension peut être exprimée par le nombre un. L'unité SI cohérente de toutes les grandeurs sans dimension, ou grandeurs de dimension un, est le nombre un, parce que l'unité est le rapport de deux unités SI identiques. La valeur de ces grandeurs est exprimée par des nombres, et l'unité « un » n'est pas mentionnée explicitement. On peut citer, comme exemple de telles grandeurs, l'indice de réfraction, la perméabilité relative ou le coefficient de frottement. D'autres grandeurs sont définies comme un produit assez complexe et sans dimension de grandeurs habituelles. Par exemple, parmi les «nombres

Le CIPM, reconnaissant l'importance particulière des unités dans le domaine de la santé, a adopté un texte détaillé sur le sievert dans la $5^{\mathrm{e}}$ édition de la brochure sur le SI,

Recommandation 1 (CI-1984), adoptée par le CIPM (PV, 1984, 52, 31 et Metrologia, 1985, 21, 90), et Recommandation 2 (CI-2002), adoptée par le CIPM (PV, 2002, 70, $102)$, voir pp. 72 et 79 , respectivement. 
caractéristiques » citons le nombre de Reynolds $R e=\rho v l / \eta$, où $\rho$ est la masse volumique, $\eta$ la viscosité dynamique, $v$ la vitesse et $l$ la longueur. Dans tous ces cas, l'unité peut être considérée comme étant le nombre un, unité dérivée sans dimension.

Une autre classe de grandeurs sans dimension est celle des nombres qui servent à indiquer un comptage, comme le nombre de molécules, la dégénérescence (nombre de niveaux d'énergie) ou la fonction de partition en thermodynamique statistique (nombre d'états thermiques accessibles). Toutes ces grandeurs de comptage sont décrites comme étant sans dimension, ou de dimension un, et ont pour unité l'unité SI un, même si l'unité des grandeurs de comptage ne peut pas être décrite comme une unité dérivée exprimée en unités de base du SI. Pour ces grandeurs, l'unité un pourrait être considérée comme une autre unité de base.

Dans quelques cas, cependant, cette unité se voit attribuer un nom spécial, en vue de faciliter l'identification de la grandeur concernée. C'est le cas du radian et du stéradian. Le radian et le stéradian ont reçu de la CGPM un nom spécial pour l'unité dérivée cohérente un, afin d'exprimer les valeurs de l'angle plan et de l'angle solide, respectivement, et figurent donc au tableau 3. 


\section{$3 \quad$ Multiples et sous-multiples décimaux des unités SI}

\subsection{Préfixes SI}

Les préfixes SI représentent strictement des puissances de 10 . Ils ne doivent pas être utilisés pour exprimer des puissances de 2 (par exemple, un kilobit représente 1000 bits et non 1024 bits). Les préfixes adoptés par la CEI pour les puissances binaires sont publiés dans la norme internationale CEI 60027-2 : 2005, $3^{\mathrm{e}}$ édition, Symboles littéraux à utiliser en électrotechnique Partie 2: Télécommunications et électronique. Les noms et symboles des préfixes correspondant à $2^{10}, 2^{20}, 2^{30}, 2^{40}, 2^{50}$, et $2^{60}$ sont, respectivement : kibi, $\mathrm{Ki}$; mébi, Mi ; gibi, Gi ; tébi, Ti ; pébi, Pi ; et exbi, Ei. Ainsi, par exemple, un kibioctet s'écrit :

$1 \mathrm{KiB}=2{ }^{10} \mathrm{~B}=1024 \mathrm{~B}$, où $\mathrm{B}$ désigne l'octet. Bien que ces préfixes n'appartiennent pas au SI, ils doivent être utilisés en informatique afin d'éviter un usage incorrect des préfixes SI.

Exemples d'utilisation de préfixes : pm (picomètre) mmol (millimole) $\mathrm{G} \Omega($ gigaohm) THz (térahertz)
Les symboles des préfixes sont écrits en romain, comme les symboles d'unités, quelle que soit la police employée par ailleurs. Ils sont attachés aux symboles d'unités, sans espace entre le symbole du préfixe et celui de l'unité. À l'exception de da (déca), $\mathrm{h}$ (hecto), et k (kilo), tous les symboles des préfixes des multiples sont en majuscules, et tous les symboles des préfixes des sous-multiples sont en minuscules. Tous les noms de préfixes sont en minuscules, sauf en début de phrase.

Le groupe formé d'un symbole de préfixe et d'un symbole d'unité constitue un nouveau symbole d'unité inséparable (formant un multiple ou sous-multiple de l'unité en question) qui peut être élevé à une puissance positive ou négative, et qui peut être combiné à d'autres symboles d'unités pour former des symboles d'unités composés.

Exemples:

$$
\begin{aligned}
& 2,3 \mathrm{~cm}^{3}=2,3(\mathrm{~cm})^{3}=2,3\left(10^{-2} \mathrm{~m}\right)^{3}=2,3 \times 10^{-6} \mathrm{~m}^{3} \\
& 1 \mathrm{~cm}^{-1}=1(\mathrm{~cm})^{-1}=1\left(10^{-2} \mathrm{~m}\right)^{-1}=10^{2} \mathrm{~m}^{-1}=100 \mathrm{~m}^{-1} \\
& 1 \mathrm{~V} / \mathrm{cm}=(1 \mathrm{~V}) /\left(10^{-2} \mathrm{~m}\right)=10^{2} \mathrm{~V} / \mathrm{m}=100 \mathrm{~V} / \mathrm{m} \\
& 5000 \mu \mathrm{s}^{-1}=5000(\mu \mathrm{s})^{-1}=5000\left(10^{-6} \mathrm{~s}\right)^{-1}=5 \times 10^{9} \mathrm{~s}^{-1}
\end{aligned}
$$


De même, les noms de préfixes ne sont pas séparés des noms d'unités auxquels ils sont attachés. Ainsi, par exemple, millimètre, micropascal, et méganewton s'écrivent en un seul mot.

Les symboles de préfixes composés, c'est-à-dire les symboles de préfixes formés par juxtaposition de deux ou plusieurs symboles de préfixes, sont interdits. Cette règle s'applique aussi aux noms de préfixes composés.

Les symboles de préfixes ne peuvent pas être utilisés seuls ou attachés au nombre 1, le symbole de l'unité un. De même, les noms de préfixes ne peuvent pas être attachés au nom de l'unité un, c'est-à-dire au mot un.

Les noms et symboles de préfixes sont utilisés avec un certain nombre d'unités en dehors du SI (voir chapitre 5), mais ils ne sont jamais utilisés avec les unités de temps: minute, min; heure, $\mathrm{h}$; jour, $\mathrm{d}$. Les astronomes utilisent toutefois la milliseconde d'arc (ou de degré), symbole mas, et la microseconde d'arc, symbole $\mu$ as, comme unité de mesure des très petits angles.

\subsection{Le kilogramme}

Parmi les unités de base du Système international, l'unité de masse est la seule dont le nom, pour des raisons historiques, contienne un préfixe. Les noms et les symboles des multiples et sous-multiples décimaux de l'unité de masse sont formés par l'adjonction

$10^{-6} \mathrm{~kg}=1 \mathrm{mg}$ mais pas $1 \mu \mathrm{kg}$ (microkilogramme). de noms de préfixes au mot " gramme » et de symboles de ces préfixes au symbole de l'unité « g » (CIPM, 1967, Recommandation 2; PV, 35, 29 et Metrologia, 1968, 4, $45)$.

manomètre) mais pas

$\mathrm{m} \mu \mathrm{m}$ (millimicromètre)

Le nombre d'atomes de plomb dans l'échantillon est égal à $N(\mathrm{~Pb})=5 \times 10^{6}$, mais pas $N(\mathrm{~Pb})=5 \mathrm{M}$, où $\mathrm{M}$ représenterait le préfixe méga. 


\section{$4 \quad$ Unités en dehors du SI}

Le Système international d'unités, SI, est un système d'unités, adopté par la Conférence générale, qui fournit les unités de référence approuvées au niveau international en fonction desquelles toutes les autres unités sont définies. L'usage du SI est recommandé dans les sciences, la technologie, les sciences de l'ingénieur et le commerce. Les unités de base du SI et les unités SI dérivées cohérentes, y compris celles ayant des noms spéciaux, ont l'avantage considérable de former un ensemble cohérent. En conséquence, il n'est pas nécessaire d'effectuer des conversions d'unités quand on affecte des valeurs particulières aux grandeurs dans les équations aux grandeurs. Parce que le SI est le seul système d'unités reconnu au niveau mondial, il a l'avantage manifeste d'établir un langage universel. Enfin, si tous utilisent ce système, il simplifiera l'enseignement des sciences et de la technologie à la prochaine génération.

Il est néanmoins reconnu que certaines unités en dehors du SI sont encore utilisées dans les publications scientifiques, techniques et commerciales, et qu'elles continueront à l'être encore pendant de nombreuses années. Certaines unités en dehors du SI sont importantes, d'un point de vue historique, dans la littérature traditionnelle. D'autres unités en dehors du SI, comme les unités de temps et d'angle, sont tellement ancrées dans l'histoire et la culture humaine qu'elles continueront à être utilisées dans un avenir prévisible. D'autre part, les scientifiques doivent avoir la liberté d'utiliser parfois des unités en dehors du SI, s'ils y trouvent un avantage particulier dans leur travail. Citons par exemple l'utilisation des unités CGS de Gauss pour la théorie de l'électromagnétisme appliquée à l'électrodynamique quantique et à la relativité. C'est pourquoi il est utile d'établir une liste des unités en dehors du SI les plus importantes, ce qui est fait dans les tableaux ci-dessous. Cependant, lorsqu'on utilise ces unités, il faut bien comprendre que l'on perd les avantages du SI.

L'inclusion d'unités en dehors du SI dans ce texte n'implique pas que leur usage est encouragé. Pour les raisons que nous avons déjà invoquées, l'usage des unités SI est en général préférable. Il est aussi souhaitable d'éviter d'utiliser conjointement des unités en dehors du SI et des unités SI ; en particulier, la combinaison d'unités en dehors du SI et d'unités SI pour former des unités composées doit être restreinte à des cas particuliers afin de ne pas compromettre les avantages du SI. Enfin, il est de bon usage de définir les unités en dehors du SI figurant aux tableaux 7, 8 et 9, en fonction des unités SI correspondantes.

\subsection{Unités en dehors du SI en usage avec le SI et unités fondées sur des constantes fondamentales}

Le Comité international a révisé en 2004 la classification des unités en dehors du SI publiée dans la $7^{\mathrm{e}}$ édition de la brochure sur le SI. Le tableau 6 donne une liste des unités en dehors du SI dont l'usage avec le Système international est accepté par le CIPM, parce qu'elles sont largement utilisées dans la vie quotidienne. Leur utilisation 
pourrait se poursuivre indéfiniment, et chacune de ces unités a une définition exacte en unités SI. Les tableaux 7, 8 et 9 contiennent des unités utilisées uniquement dans des circonstances particulières. Les unités du tableau 7 sont liées à des constantes fondamentales et leur valeur doit être déterminée de manière expérimentale. Les tableaux 8 et 9 contiennent des unités qui ont une valeur définie quand elles sont exprimées en unités SI, et qui sont utilisées dans des circonstances particulières afin de satisfaire aux besoins dans les domaines commerciaux et légaux, ou à des intérêts scientifiques particuliers. Il est probable que ces unités continueront à être utilisées encore pendant de nombreuses années. Beaucoup de ces unités sont importantes pour l'interprétation des anciens textes scientifiques. Les tableaux 6, 7, 8 et 9 sont présentés ci-dessous.

Le tableau 6 contient les unités traditionnelles de temps et d'angle. Il contient aussi l'hectare, le litre et la tonne, qui sont tous d'usage courant au niveau mondial, et qui diffèrent des unités SI cohérentes correspondantes d'un facteur égal à une puissance entière de dix. Les préfixes SI sont utilisés avec plusieurs de ces unités, mais pas avec les unités de temps.

Tableau 6. Unités en dehors du SI dont l'usage est accepté avec le SI

\begin{tabular}{|c|c|c|c|}
\hline Grandeur & Nom de l'unité & Symbole de l'unité & Valeur en unités SI \\
\hline \multirow[t]{3}{*}{ temps } & minute & $\min$ & $1 \mathrm{~min}=60 \mathrm{~s}$ \\
\hline & heure $^{(a)}$ & $\mathrm{h}$ & $1 \mathrm{~h}=60 \mathrm{~min}=3600 \mathrm{~s}$ \\
\hline & jour & $\mathrm{d}$ & $1 \mathrm{~d}=24 \mathrm{~h}=86400 \mathrm{~s}$ \\
\hline \multirow[t]{3}{*}{ angle plan } & degré $^{(b, c)}$ & o & $1^{\circ}=(\pi / 180) \mathrm{rad}$ \\
\hline & minute & ' & $1^{\prime}=(1 / 60)^{\circ}=(\pi / 10800) \mathrm{rad}$ \\
\hline & seconde ${ }^{(d)}$ & $"$ & $1^{\prime \prime}=(1 / 60)^{\prime}=(\pi / 648000) \mathrm{rad}$ \\
\hline superficie & hectare $^{(e)}$ & ha & $1 \mathrm{ha}=1 \mathrm{hm}^{2}=10^{4} \mathrm{~m}^{2}$ \\
\hline volume & litre $^{(f)}$ & $\mathrm{L}, 1$ & $1 \mathrm{~L}=1 \mathrm{l}=1 \mathrm{dm}^{3}=10^{3} \mathrm{~cm}^{3}=10^{-3} \mathrm{~m}^{3}$ \\
\hline masse & tonne ${ }^{(g)}$ & $\mathrm{t}$ & $1 \mathrm{t}=10^{3} \mathrm{~kg}$ \\
\hline
\end{tabular}

(a) Le symbole de cette unité figure dans la Résolution 7 de la $9^{\mathrm{e}}$ CGPM (1948; CR, 70).

(b) La norme ISO 31 recommande que le degré soit subdivisé de manière décimale plutôt qu'en utilisant la minute et la seconde. Pour la navigation et la topographie, toutefois, l'avantage d'utiliser la minute tient au fait qu'une minute de latitude à la surface de la Terre correspond (approximativement) à un mille marin.

(c) Le gon, parfois appelé grade, est une autre unité d'angle plan définie comme étant égale à $(\pi / 200)$ rad. Un angle droit comporte donc 100 gons ou 100 grades. Le gon ou le grade peuvent être utiles dans le domaine de la navigation parce que la distance entre le pôle et l'Équateur à la surface de la Terre est égale environ à $10000 \mathrm{~km}, 1 \mathrm{~km}$ à la surface de la Terre sous-tend donc un angle d'un centigon ou d'un centigrade au centre de la Terre. Le gon et le grade sont toutefois très rarement utilisés.

(d) En astronomie, les petits angles sont mesurés en seconde d'arc (c'est-à-dire en secondes d'angle plan), en milli-, micro- ou picosecondes d'arc (symbole as ou ", mas, $\mu$ as et pas, respectivement). L'arcseconde ou la seconde de degré sont d'autres noms pour la seconde d'angle plan.

(e) L'unité hectare et son symbole ha furent adoptés par le Comité international en 1879 (PV, $1879,41)$. L'hectare est utilisé pour exprimer des superficies agraires.

(f) Le litre et son symbole 1 (en minuscule) furent adoptés par le Comité international en 1879 (PV, 1879, 41). Le symbole L (en capitale) fut adopté par la $16^{\mathrm{e}}$ Conférence générale (1979, Résolution 6 ; CR, 101 et Metrologia, 1980, 16, 56-57), comme alternative pour éviter le risque de confusion entre la lettre 1 et le chiffre un, 1.

(g) La tonne et son symbole t furent adoptés par le Comité international en 1879 (PV, 1879, 41). Dans les pays de langue anglaise, cette unité est généralement désignée sous le nom «tonne métrique ». 
Le tableau 7 contient des unités dont les valeurs en unités SI ne peuvent être connues que par détermination expérimentale, avec une incertitude associée. À l'exception de l'unité astronomique, toutes les unités du tableau 7 sont liées à des constantes fondamentales de la physique. Le Comité international a accepté l'usage avec le SI des trois premières unités de ce tableau: les unités en dehors du SI électronvolt (symbole eV), dalton (symbole Da) ou unité de masse atomique unifiée (symbole u), et l'unité astronomique (symbole ua). Les unités du tableau 7 jouent un rôle important dans un certain nombre de domaines spécialisés, dont les résultats de mesure et les calculs sont plus commodément et utilement exprimés au moyen de ces unités. Pour l'électronvolt et le dalton, les valeurs dépendent de la charge électrique élémentaire $e$ et de la constante d'Avogadro $N_{\mathrm{A}}$, respectivement.

Il existe de nombreuses autres unités de cette nature, car il y a de nombreux domaines dans lesquels il est plus commode d'exprimer les résultats d'observations expérimentales ou de calculs théoriques au moyen des constantes fondamentales de la nature. Les deux systèmes d'unités les plus importants fondés sur des constantes fondamentales sont le système d'unités naturelles (u.n.) utilisé dans le domaine de la physique des hautes énergies et des particules, et le système d'unités atomiques (u.a.) utilisé en physique atomique et en chimie quantique. Dans le système d'unités naturelles, les grandeurs de base en mécanique sont la vitesse, l'action et la masse, dont les unités de base sont la vitesse de la lumière dans le vide $c_{0}$, la constante de Planck $h$ divisée par $2 \pi$, appelée constante de Planck réduite avec le symbole $\hbar$, et la masse de l'électron $m_{\mathrm{e}}$, respectivement. En général ces unités n'ont pas reçu de nom spécial ou de symbole particulier, mais sont simplement appelées unité naturelle de vitesse, symbole $c_{0}$, unité naturelle d'action, symbole $\hbar$, et unité naturelle de masse, symbole $m_{\mathrm{e}}$. Dans ce système, le temps est une grandeur dérivée et l'unité naturelle de temps est une unité dérivée égale à la combinaison d'unités de base $\hbar / m_{\mathrm{e}} c_{0}{ }^{2}$. De même dans le système d'unités atomiques, n'importe quel ensemble de quatre des cinq grandeurs charge, masse, action, longueur et énergie est considéré comme un ensemble de grandeurs de base. Les unités de base sont $e$ pour la charge électrique élémentaire, $m_{\mathrm{e}}$ pour la masse de l'électron, $\hbar$ pour l'action, $a_{0}$ (ou bohr) pour le rayon de Bohr, et $E_{\mathrm{h}}$ (ou hartree) pour l'énergie de Hartree, respectivement. Dans ce système, le temps est aussi une grandeur dérivée et l'unité atomique de temps est une unité dérivée, égale à $\hbar / E_{\mathrm{h}}$. Notons que $a_{0}=\left(\alpha / 4 \pi R_{\infty}\right)$, où $\alpha$ est la constante de structure fine et $R_{\infty}$ est la constante de Rydberg, et que $E_{\mathrm{h}}=e^{2} /\left(4 \pi \varepsilon_{0} a_{0}\right)=2 R_{\infty} h c_{0}=\alpha^{2} m_{\mathrm{e}} c_{0}^{2}$, où $\varepsilon_{0}$ est la constante électrique (la permittivité du vide) ; $\varepsilon_{0}$ a une valeur exacte dans le SI.

Pour information, ces dix unités naturelles et atomiques et leur valeur en unité SI figurent au tableau 7. Parce que les systèmes de grandeurs sur lesquelles ces unités sont fondées diffèrent vraiment de manière fondamentale de celles du SI, elles ne sont généralement pas utilisées avec le SI, et le Comité international n'a pas officiellement approuvé leur usage avec le Système international. Pour une bonne compréhension, le résultat final d'une mesure ou d'un calcul exprimé en unités naturelles ou atomiques doit aussi toujours être exprimé dans l'unité SI correspondante. Les unités naturelles et les unités atomiques sont utilisées uniquement dans les domaines particuliers de la physique des particules, de la physique atomique et de la chimie quantique. Les incertitudes-type sur les derniers chiffres significatifs figurent entre parenthèses après chaque valeur numérique. 
Tableau 7. Unités en dehors du SI dont la valeur en unités SI est obtenue expérimentalement

\begin{tabular}{llll}
\hline Grandeur & Nom de l'unité & Symbole de l'unité & Valeur en unités SI ${ }^{(a)}$ \\
\hline & & Unités en usage avec le SI \\
\multirow{2}{*}{ énergie } & électronvolt $^{(b)}$ & $\mathrm{eV}$ & $1 \mathrm{eV}=1,60217653(14) \times 10^{-19} \mathrm{~J}$ \\
masse & dalton, $^{(c)}$ & $\mathrm{Da}$ & $1 \mathrm{Da}=1,66053886(28) \times 10^{-27} \mathrm{~kg}$ \\
& unité de masse atomique unifiée $^{*} \mathrm{u}$ & $1 \mathrm{u}=1 \mathrm{Da}$ \\
longueur & unité astronomique $^{(d)}$ & $\mathrm{ua}$ & $1 \mathrm{ua}=1,49597870691(6) \times 10^{11} \mathrm{~m}$
\end{tabular}

\section{Unités naturelles}

\begin{tabular}{llll} 
vitesse & \multicolumn{2}{l}{$\begin{array}{l}\text { unité naturelle de vitesse } \\
\text { (vitesse de la lumière dans le vide) }\end{array}$} & $299792458 \mathrm{~m} / \mathrm{s}$ (exactement) \\
action & $\begin{array}{l}c_{0} \\
\text { unité naturelle d'action } \\
\text { (constante de Planck réduite) }\end{array}$ & $\hbar$ & $1,05457168(18) \times 10^{-34} \mathrm{~J} \mathrm{~s}$ \\
masse & $\begin{array}{l}\text { unité naturelle de masse } \\
\text { (masse de l'électron) }\end{array}$ & $m_{\mathrm{e}}$ & $9,1093826(16) \times 10^{-31} \mathrm{~kg}$ \\
temps & $\begin{array}{l}\text { unité naturelle de temps } \\
\text { tem }\end{array}$ & $\hbar / m_{\mathrm{e}} c_{0}{ }^{2}$ & $1,2880886677(86) \times 10^{-21} \mathrm{~s}$
\end{tabular}

\section{Unités atomiques}

$\begin{array}{llll}\text { charge } & \begin{array}{l}\text { unité atomique de charge } \\ \text { (charge électrique élémentaire) } \\ \text { masse }\end{array} & e & 1,60217653(14) \times 10^{-19} \mathrm{C} \\ \text { unité atomique de masse } & m_{\mathrm{e}} & 9,1093826(16) \times 10^{-31} \mathrm{~kg} \\ \text { (masse de l'électron) } & \begin{array}{l}\text { unité atomique d'action } \\ \text { (constante de Planck réduite) }\end{array} & \hbar & 1,05457168(18) \times 10^{-34} \mathrm{~J} \mathrm{~s} \\ \text { longueur } & \begin{array}{l}\text { unité atomique de longueur } \\ \text { bohr (rayon de Bohr) }\end{array} & a_{0} & 0,5291772108(18) \times 10^{-10} \mathrm{~m} \\ \text { temps } & \begin{array}{l}\text { unité atomique d'énergie, } \\ \text { hartree (énergie de Hartree) } \\ \text { unité atomique de temps }\end{array} & E_{\mathrm{h}} & 4,35974417(75) \times 10^{-18} \mathrm{~J} \\ & \hbar / E_{\mathrm{h}} & 2,418884326505(16) \times 10^{-17} \mathrm{~s}\end{array}$

(a) Les valeurs en unités SI de toutes les unités de ce tableau, à l'exception de l'unité astronomique, proviennent de la liste des valeurs des constantes fondamentales recommandées par CODATA en 2002, publiée par P.J. Mohr et B.N. Taylor, Rev. Mod. Phys., 2005, 77, 1-107. L'incertitude-type sur les deux derniers chiffres est donnée entre parenthèses (voir 5.3.5, p. 45).

(b) L'électronvolt est l'énergie cinétique acquise par un électron après traversée d'une différence de potentiel de $1 \mathrm{~V}$ dans le vide. L'électronvolt est souvent combiné aux préfixes SI.

(c) Le dalton (Da) et l'unité de masse atomique unifiée (u) sont d'autres noms (et symboles) pour la même unité, égale à $1 / 12$ de la masse de l'atome de ${ }^{12} \mathrm{C}$ libre, au repos et dans son état fondamental. Le dalton est souvent combiné à des préfixes SI, par exemple pour exprimer la masse de grosses molécules en kilodaltons, $\mathrm{kDa}$, ou mégadaltons, $\mathrm{MDa}$, et pour exprimer la valeur de petites différences de masse d'atomes ou de molécules en nanodaltons, $\mathrm{nDa}$, voire picodaltons, $\mathrm{pDa}$.

(d) L'unité astronomique est égale approximativement à la distance moyenne entre le Soleil et la Terre. C'est le rayon d'une orbite newtonienne circulaire non perturbée autour du Soleil d'une particule de masse infinitésimale, se déplaçant à une vitesse moyenne de 0,017 20209895 radians par jour (encore appelée constante de Gauss). La valeur de l'unité astronomique est donnée dans l'IERS Conventions 2003 (D.D. McCarthy et G. Petit eds., IERS Technical Note 32, Frankfurt am Main : Verlag des Bundesamts für Kartographie und Geodäsie, 2004, 12). La valeur de l'unité astronomique en mètres provient des JPL ephemerides DE403 (Standish E.M., Report of the IAU WGAS Sub-Group on Numerical Standards, Highlights of Astronomy, Appenzeller ed., Dordrecht : Kluwer Academic Publishers, 1995, 180-184). 
Les tableaux 8 et 9 contiennent des unités en dehors du SI utilisées afin de répondre aux besoins spécifiques de certains groupes, pour différentes raisons. Bien qu'il soit préférable d'utiliser les unités SI pour les raisons déjà invoquées précédemment, les auteurs qui voient un avantage particulier à utiliser ces unités en dehors du SI doivent être libres de le faire s'ils les considèrent mieux appropriées à leurs besoins. Puisque, toutefois, les unités SI sont le fondement international d'après lequel toutes les autres unités sont définies, ceux qui utilisent les unités des tableaux 8 et 9 doivent toujours mentionner leur définition en unités SI.

Le tableau 8 mentionne aussi les unités des grandeurs logarithmiques, le néper, le bel et le décibel. Ce sont des unités sans dimension, d'une nature quelque peu différente des autres unités sans dimension, et certains scientifiques considèrent que l'on ne devrait même pas les appeler unités. Elles sont utilisées pour véhiculer des informations sur la nature logarithmique de rapports de grandeurs. Le néper, $\mathrm{Np}$, est utilisé pour exprimer la valeur de logarithmes néperiens (ou naturels) de rapports de grandeurs, $\ln =\log _{\mathrm{e}}$. Le bel et le décibel, $\mathrm{B}$ et $\mathrm{dB}, 1 \mathrm{~dB}=(1 / 10) \mathrm{B}$, sont utilisés pour exprimer la valeur de logarithmes de base 10 de rapports de grandeurs, $\lg =\log _{10}$. La manière dont ces unités sont interprétées est décrite dans les notes $(g)$ et $(h)$ du tableau 8. Il est rarement nécessaire de donner une valeur numérique à ces unités. L'usage des unités néper, bel, et décibel avec le SI a été accepté par le Comité international, mais ces unités ne sont pas considérées comme des unités SI.

Les préfixes SI sont utilisés avec deux des unités du tableau 8 , à savoir avec le bar (par exemple millibar, mbar) et le bel, en particulier le décibel, dB. Le décibel est explicitement mentionné dans le tableau parce que le bel est rarement utilisé sans ce préfixe.

Tableau 8. Autres unités en dehors du SI

\begin{tabular}{|c|c|c|c|}
\hline Grandeur & Nom de l'unité & Symbole de l'unité & Valeur en unités SI \\
\hline pression & $\begin{array}{l}\text { bar }^{(a)} \\
\text { millimètre de } \\
\text { mercure }^{(b)}\end{array}$ & $\begin{array}{l}\text { bar } \\
\mathrm{mmHg}\end{array}$ & $\begin{array}{l}1 \mathrm{bar}=0,1 \mathrm{MPa}=100 \mathrm{kPa}=10^{5} \mathrm{~Pa} \\
1 \mathrm{mmHg}=133,322 \mathrm{~Pa}\end{array}$ \\
\hline $\begin{array}{l}\text { longueur } \\
\text { distance } \\
\text { superficie } \\
\text { vitesse } \\
\text { logarithme } \\
\text { d'un rapport }\end{array}$ & $\begin{array}{l}\text { ångström }^{(c)} \\
\text { mille marin } \\
\text { barn }^{(e)} \\
\text { noeud }^{(f)} \\
\text { néper }^{(g, i)} \\
\text { bel }^{(h, i)} \\
\text { décibel }^{(h, i)}\end{array}$ & $\begin{array}{l}\AA \\
\mathrm{M} \\
\mathrm{b} \\
\mathrm{kn} \\
\mathrm{Np} \\
\mathrm{B} \\
\mathrm{dB}\end{array}$ & $\begin{array}{l}1 \AA=0,1 \mathrm{~nm}=100 \mathrm{pm}=10^{-10} \mathrm{~m} \\
1 \mathrm{M}=1852 \mathrm{~m} \\
1 \mathrm{~b}=100 \mathrm{fm}^{2}=\left(10^{-12} \mathrm{~cm}\right)^{2}=10^{-28} \mathrm{~m}^{2} \\
1 \mathrm{kn}=(1852 / 3600) \mathrm{m} / \mathrm{s} \\
\text { [voir note }(j) \text { au sujet de la valeur } \\
\text { numérique du néper, du bel et du décibel] }\end{array}$ \\
\hline
\end{tabular}

(a) Le bar et son symbole sont inclus dans la Résolution 7 de la $9^{\mathrm{e}}$ CGPM (1948; CR, 70). Depuis 1982 , toutes les données thermodynamiques sont tabulées pour la pression normale de un bar. Avant 1982, la pression normale était l'atmosphère normale, égale à 1,01325 bar, ou $101325 \mathrm{~Pa}$.

(b) Le millimètre de mercure est l'unité légale pour la mesure de la pression sanguine dans certains pays.

(c) L'ångström est largement utilisé dans le domaine de la cristallographie aux rayons $\mathrm{x}$ et en chimie de la structure parce que la longueur des liaisons chimiques se situe dans le domaine compris entre 1 et 3 ångströms. L'ångström n'a toutefois pas été sanctionné par le Comité international ni par la Conférence générale.

(d) Le mille marin est une unité employée en navigation maritime et aérienne pour exprimer les distances. Cette valeur fut adoptée par convention par la Première Conférence hydrographique internationale extraordinaire, Monaco, 1929, sous le nom de «mille marin international ». Il n'existe pas de symbole convenu au niveau international, mais les symboles $\mathrm{M}, \mathrm{NM}, \mathrm{Nm}$ et nmi sont utilisés; seul le symbole $M$ est indiqué dans le tableau 8 . Cette unité avait été choisie 
à l'origine, et continue à être utilisée, parce qu'un mille marin à la surface de la Terre est soustendu approximativement par une minute d'angle au centre de la Terre, ce qui est commode quand la latitude et la longitude sont mesurées en degrés et minutes d'angle.

(e) Le barn est une unité de superficie employée en physique nucléaire pour exprimer les sections efficaces.

(f) Le nœud correspond à un mille marin par heure. Il n'existe pas de symbole convenu au niveau international, mais le symbole kn est habituellement utilisé.

(g) L'égalité $L_{A}=n \mathrm{~Np}$ (où $n$ est un nombre) est comprise comme signifiant que $\ln \left(A_{2} / A_{1}\right)=n$. Ainsi lorsque $L_{A}=1 \mathrm{~Np}, A_{2} / A_{1}=\mathrm{e}$. Le symbole $A$ est utilisé ici pour désigner l'amplitude d'un signal sinusoïdal, et $L_{A}$ est alors appelé logarithme néperien du rapport d'amplitude, ou différence de niveau d'amplitude néperien.

(h) L'égalité $L_{X}=m \mathrm{~dB}=(m / 10) \mathrm{B}$ (où $m$ est un nombre) est comprise comme signifiant que $\lg \left(X / X_{0}\right)=m / 10$. Ainsi lorsque $L_{X}=1 \mathrm{~B}, X / X_{0}=10$, et lorsque $L_{X}=1 \mathrm{~dB}, X / X_{0}=10^{1 / 10}$. Si $X$ désigne un signal quadratique moyen ou une grandeur de type puissance, $L_{X}$ est appelé niveau de puissance par référence à $X_{0}$.

(i) Lorsque l'on utilise ces unités, il est important de préciser quelle est la nature de la grandeur en question et la valeur de référence utilisée. Ces unités ne sont pas des unités du SI, mais leur usage avec le SI a été accepté par le Comité international.

(j) Il est rarement nécessaire de préciser les valeurs numériques du néper, du bel et du décibel (et la relation du bel et du décibel au néper). Ceci dépend de la manière dont les grandeurs logarithmiques sont définies.

Le tableau 9 diffère du tableau 8 en ce que les unités mentionnées au tableau 9 sont reliées aux anciennes unités du système CGS (centimètre, gramme, seconde), y compris les unités électriques CGS. Dans le domaine de la mécanique, le système d'unités CGS était fondé sur trois grandeurs et leurs unités de base correspondantes : le centimètre, le gramme et la seconde. Les unités électriques CGS continuent à être dérivées de ces trois unités de base seulement, au moyen d'équations différentes de celles utilisées dans le SI. Cela pouvant être réalisé de différentes façons, plusieurs systèmes différents ont été établis : le système CGS-UES (électrostatique), le système CGS-UEM (électromagnétique) et le système d'unités CGS de Gauss. Il a toujours été reconnu que le système CGS de Gauss, en particulier, présente des avantages dans certains domaines de la physique, comme l'électrodynamique classique et relativiste ( $9^{\mathrm{e}}$ CGPM, 1948, Résolution 6). Le tableau 9 donne les relations entre les unités CGS et le SI, ainsi que la liste des unités CGS qui ont reçu un nom spécial. Comme pour les unités du tableau 8 , les préfixes SI sont utilisés avec plusieurs de ces unités (par exemple millidyne, mdyn ; milligauss, $\mathrm{mG}$ etc.).

Tableau 9. Unités en dehors du SI associées aux systèmes d'unités CGS et CGS de Gauss

\begin{tabular}{|c|c|c|c|}
\hline Grandeur & Nom de l'unité & Symbole de l'unité & Valeur en unités SI \\
\hline énergie & $\operatorname{erg}^{(a)}$ & erg & $1 \mathrm{erg}=10^{-7} \mathrm{~J}$ \\
\hline force & dyne $^{(a)}$ & dyn & $1 \mathrm{dyn}=10^{-5} \mathrm{~N}$ \\
\hline viscosité dynamique & poise $^{(a)}$ & $\mathrm{P}$ & $1 \mathrm{P}=1 \mathrm{dyn} \mathrm{s} \mathrm{cm}{ }^{-2}=0,1 \mathrm{~Pa} \mathrm{~s}$ \\
\hline viscosité cinématique & stokes & St & $1 \mathrm{St}=1 \mathrm{~cm}^{2} \mathrm{~s}^{-1}=10^{-4} \mathrm{~m}^{2} \mathrm{~s}^{-1}$ \\
\hline luminance lumineuse & stilb $^{(a)}$ & $\mathrm{sb}$ & $1 \mathrm{sb}=1 \mathrm{~cd} \mathrm{~cm}^{-2}=10^{4} \mathrm{~cd} \mathrm{~m}^{-2}$ \\
\hline éclairement lumineux & phot & $\mathrm{ph}$ & $1 \mathrm{ph}=1 \mathrm{~cd} \mathrm{sr} \mathrm{cm}^{-2}=10^{4} \mathrm{~lx}$ \\
\hline accélération & gal $^{(b)}$ & Gal & $1 \mathrm{Gal}=1 \mathrm{~cm} \mathrm{~s}^{-2}=10^{-2} \mathrm{~m} \mathrm{~s}^{-2}$ \\
\hline flux magnétique & maxwell ${ }^{(c)}$ & $\mathrm{Mx}$ & $1 \mathrm{Mx}=1 \mathrm{G} \mathrm{cm}^{2}=10^{-8} \mathrm{~Wb}$ \\
\hline induction magnétique & gauss $^{(c)}$ & G & $1 \mathrm{G}=1 \mathrm{Mx} / \mathrm{cm}^{2}=10^{-4} \mathrm{~T}$ \\
\hline champ magnétique & œrsted $^{(c)}$ & Oe & $1 \mathrm{Oe} \hat{=}\left(10^{3} / 4 \pi\right) \mathrm{A} \mathrm{m}^{-1}$ \\
\hline
\end{tabular}


(a) Cette unité et son symbole sont inclus dans la Résolution 7 de la $9^{\mathrm{e}}$ CGPM (1948; CR, 70).

(b) Le gal est une unité employée en géodésie et géophysique pour exprimer l'accélération due à la pesanteur.

(c) Ces unités font partie du système CGS tri-dimensionnel "électromagnétique », fondé sur des équations aux grandeurs non rationalisées. Elles doivent donc être comparées avec soin aux unités correspondantes du Système international qui sont fondées sur des équations rationalisées à quatre dimensions et quatre grandeurs en électromagnétisme. Le flux magnétique $\Phi$ et l'induction magnétique $B$ sont définis par des équations similaires dans le système CGS et dans le SI, ce qui permet de relier les unités correspondantes dans le tableau. Cependant, le champ magnétique non rationalisé $H$ (non rationalisé) est égal à $4 \pi \times H$ (rationalisé). Le symbole d'équivalence $\hat{=}$ est utilisé pour indiquer que lorsque $H$ (non rationalisé $)=1$ Oe, $H$ (rationalisé $)=\left(10^{3} / 4 \pi\right) \mathrm{A} \mathrm{m}^{-1}$.

\subsection{Autres unités en dehors du SI dont l'usage n'est pas recommandé}

Il existe de nombreuses unités en dehors du SI, trop nombreuses pour être citées ici, qui présentent soit un intérêt historique, ou qui sont encore utilisées dans un domaine spécialisé (comme le baril de pétrole) ou dans certains pays (comme le pouce, le pied ou le yard). Le Comité international ne voit aucune raison de continuer à utiliser ces unités dans les travaux scientifiques et techniques modernes. Cependant, il est important de connaître la relation entre ces unités et les unités SI correspondantes, et ceci restera vrai encore de nombreuses années. Le Comité international a donc décidé de compiler une liste des facteurs de conversion en unités SI d'unités de ce type. Cette liste peut être consultée sur le site Web du BIPM à l'adresse :

www.bipm.org/fr/si/si brochure/chapter4/conversion factors.html. 


\section{$5 \quad$ Règles d'écriture des noms et symboles d'unités et expression des valeurs des grandeurs}

Les principes généraux concernant l'écriture des symboles des unités et des nombres furent d'abord proposés par la $9^{\mathrm{e}}$ CGPM (1948, Résolution 7). Ils furent ensuite adoptés et mis en forme par l'ISO, la CEI et par d'autres organisations internationales. Il en résulte maintenant un consensus général sur la manière dont les symboles et noms d'unités, y compris les symboles et noms de préfixes, ainsi que les symboles et les valeurs des grandeurs, doivent être exprimés. Le respect de ces règles et des conventions de style, dont les plus importantes sont présentées dans ce chapitre, aide à la lisibilité des articles scientifiques et techniques.

\subsection{Symboles des unités}

Les symboles des unités sont imprimés en caractères romains (droits), quelle que soit la police employée dans le texte où ils figurent. En général les symboles des unités sont écrits en minuscules, mais, si le nom de l'unité dérive d'un nom propre, la $\mathrm{m}$, mètre s, seconde $\mathrm{Pa}$, pascal première lettre du symbole est majuscule.

Le symbole du litre constitue une exception à cette règle. La $16^{\mathrm{e}}$ Conférence générale (1979, Résolution 6) a approuvé l'utilisation de la lettre L en majuscule ou 1 en minuscule comme symbole du litre, afin d'éviter la confusion entre le chiffre 1 (un) et la lettre 1 .

Si l'on utilise un préfixe de multiple ou sous-multiple, celui-ci fait partie de l'unité et il précède le symbole de l'unité, sans espace entre le symbole du préfixe et le symbole de l'unité. Un préfixe n'est jamais utilisé seul et l'on n'utilise jamais de préfixes composés.

Les symboles d'unités sont des entités mathématiques et pas des abréviations. Ils ne doivent donc pas être suivis d'un point, sauf s'ils se trouvent placés à la fin d'une phrase. Ils restent invariables au pluriel et il ne faut pas mélanger des symboles avec des noms d'unités dans une même expression, puisque les noms ne sont pas des entités mathématiques.

Les règles classiques de multiplication ou de division algébriques s'appliquent pour former les produits et quotients de symboles d'unités. La multiplication doit être indiquée par un espace ou un point à mi-hauteur centré $(\cdot)$, pour éviter que certains préfixes soient interprétés à tort comme un symbole d'unité. La division est indiquée par une ligne horizontale, par une barre oblique (/) ou par des exposants négatifs. Lorsque l'on combine plusieurs symboles d'unités, il faut prendre soin d'éviter toute ambiguïté, par exemple en utilisant des crochets ou des parenthèses, ou des exposants négatifs. Il ne faut pas utiliser plus d'une barre oblique dans une expression donnée s'il n'y a pas de parenthèses pour lever toute ambiguïté.

Il n'est pas autorisé d'utiliser des abréviations pour les symboles et noms d'unités, Cela fait $75 \mathrm{~cm}$ de long, mais pas $75 \mathrm{~cm}$. de long $l=75 \mathrm{~cm}$,

mais pas $75 \mathrm{cms}$

coulomb par kilogramme, mais pas coulomb par $\mathrm{kg}$

\section{$\mathrm{N} \mathrm{m} \mathrm{ou} \mathrm{N} \cdot \mathrm{m}$,} pour newton mètre $\mathrm{m} / \mathrm{s}$ ou $\frac{\mathrm{m}}{\mathrm{s}}$ ou $\mathrm{m} \mathrm{s}^{-1}$, pour mètre par seconde

ms, milliseconde $\mathrm{m} \mathrm{s}$, mètre seconde

$\mathrm{m} \mathrm{kg} /\left(\mathrm{s}^{3} \mathrm{~A}\right)$, ou $\mathrm{m} \mathrm{kg} \mathrm{s}^{-3} \mathrm{~A}^{-1}$ mais pas $\mathrm{m} \mathrm{kg} / \mathrm{s}^{3} / \mathrm{A}$ comme sec (pour $\mathrm{s}$ ou seconde), $\mathrm{mm}$ car. (pour $\mathrm{mm}^{2}$ ou millimètre carré), cc (pour 
$\mathrm{cm}^{3}$ ou centimètre cube), ou mps (pour $\mathrm{m} / \mathrm{s}$ ou mètre par seconde). L'utilisation correcte des symboles des unités SI, et des unités en général, dont il a été fait mention dans les chapitres précédents de cette brochure, est obligatoire. C'est ainsi que l'on évite les ambiguïtés et les erreurs de compréhension concernant les valeurs des grandeurs.

\subsection{Noms des unités}

Les noms des unités sont imprimés en caractères romains (droits), et sont considérés comme des noms ordinaires. En français, et en anglais, les noms d'unités commencent par une minuscule (même si le symbole de l'unité commence par une majuscule), sauf s'ils se trouvent placés au début d'une phrase ou dans un titre en majuscules. Selon cette règle, l'écriture correcte du nom de l'unité dont le symbole est ${ }^{\circ} \mathrm{C}$ est « degré Celsius » (l'unité degré commence par la lettre $\mathrm{d}$ en minuscule et le

nom d'unité

joule

J

hertz $\quad \mathrm{Hz}$

mètre $\mathrm{m}$

seconde $\quad \mathrm{s}$

ampère $\mathrm{A}$

watt $\quad$ W

qualificatif «Celsius» commence par la lettre $\mathrm{C}$ en majuscule, parce que c'est un nom propre).

Bien que les valeurs des grandeurs soient généralement exprimées au moyen de nombres et de symboles d'unités, si pour une raison quelconque le nom de l'unité est mieux approprié que son symbole, il convient d'écrire en toutes lettres le nom de l'unité.

Lorsque le nom de l'unité est accolé au nom d'un préfixe d'un multiple ou sousmultiple, il n'y a pas d'espace ni de tiret entre le nom du préfixe et celui de l'unité. L'ensemble formé du nom de préfixe et de celui de l'unité constitue un seul mot (voir aussi chapitre 3, section 3.1, p. 32).

En français et en anglais, toutefois, lorsque le nom d'une unité dérivée est constitué par multiplication de noms d'unités individuelles, il convient d'utiliser un espace ou un tiret pour séparer chaque nom d'unité.

En français et en anglais, les puissances telles que « carré » ou « cube » utilisées dans les noms d'unités sont placées après le nom de l'unité. En anglais, pour les unités de superficie ou de volume on peut toutefois utiliser les termes « square » ou « cubic », avant le nom de l'unité (des exemples sont donnés à la page 131).

\subsection{Règles et conventions stylistiques servant à exprimer les valeurs des grandeurs}

\subsubsection{Valeur et valeur numérique d'une grandeur ; utilisation du calcul formel}

La valeur d'une grandeur s'exprime comme le produit d'un nombre par une unité ; le nombre qui multiplie l'unité est la valeur numérique de la grandeur exprimée au moyen de cette unité. La valeur numérique d'une grandeur dépend du choix de l'unité. Ainsi, la valeur d'une grandeur particulière est indépendante du choix de l'unité, mais la valeur numérique est différente selon l'unité choisie.

Les symboles des grandeurs sont en général formés d'une seule lettre en italique, mais ils peuvent être précisés par des informations complémentaires en indice, exposant ou entre parenthèses. Ainsi $C$ est le symbole recommandé pour la capacité thermique, $C_{\mathrm{m}}$ pour la capacité thermique molaire, $C_{\mathrm{m}, p}$ pour la capacité thermique molaire à pression constante, et $C_{\mathrm{m}, V}$ pour la capacité thermique molaire à volume constant.
$2,6 \mathrm{~m} / \mathrm{s}$

ou 2,6 mètres par seconde

milligramme,

mais pas milli-gramme

kilopascal

mais pas kilo-pascal

pascal seconde,

ou pascal-seconde

mètre par seconde carrée, centimètre carré,

millimètre cube, ampère par mètre carré,

kilogramme par mètre cube
La valeur de la vitesse d'une particule $v=\mathrm{d} x / \mathrm{d} t$ peut être exprimée par les expressions $v=25 \mathrm{~m} / \mathrm{s}=90 \mathrm{~km} / \mathrm{h}$, où 25 est la valeur numérique de la vitesse exprimée dans l'unité mètre par seconde et 90 celle exprimée dans l'unité kilomètre par heure. 
Les noms et symboles recommandés pour les grandeurs figurent dans de nombreux ouvrages de référence, comme la norme ISO 31 Grandeurs et unités, le «livre rouge » de l'UIPPA SUNAMCO, Symbols, Units and Nomenclature in Physics, et le «livre vert» de 1'UICPA, Quantities, Units and Symbols in Physical Chemistry. Toutefois, les symboles des grandeurs ne sont que recommandés, alors qu'il est obligatoire d'utiliser les symboles corrects des unités. Dans des circonstances particulières, les auteurs peuvent préférer utiliser le symbole de leur choix pour une grandeur donnée, par exemple pour éviter un conflit résultant de l'utilisation du même symbole pour deux grandeurs différentes. Il faut alors préciser clairement quelle est la signification du symbole. Le nom d'une grandeur, ou le symbole utilisé pour l'exprimer, n'oblige en aucun cas à choisir une unité en particulier.

Les symboles des unités sont traités comme des entités mathématiques. Lorsque l'on exprime la valeur d'une grandeur comme le produit d'une valeur numérique par une unité, la valeur numérique et l'unité peuvent être traitées selon les règles ordinaires de l'algèbre. Une telle démarche correspond à l'utilisation du calcul formel. Par exemple l'équation $T=293 \mathrm{~K}$ peut aussi s'écrire $T / \mathrm{K}=293$. Il est souvent commode d'écrire ainsi le quotient d'une grandeur et d'une unité en tête de colonne d'un tableau, afin que les entrées du tableau soient simplement des nombres. Par exemple, un tableau exprimant la pression de vapeur en fonction de la température, et le logarithme naturel de la pression de vapeur en fonction de la température à la puissance moins un, peut prendre la forme suivante :

\begin{tabular}{lccc}
\hline$T / \mathrm{K}$ & $10^{3} \mathrm{~K} / T$ & $p / \mathrm{MPa}$ & $\ln (p / \mathrm{MPa})$ \\
\hline 216,55 & 4,6179 & 0,5180 & $-0,6578$ \\
273,15 & 3,6610 & 3,4853 & 1,2486 \\
304,19 & 3,2874 & 7,3815 & 1,9990 \\
\hline
\end{tabular}

Les axes d'un graphique peuvent aussi être symbolisés de cette manière, afin que les graduations soient purement numériques, comme indiqué sur la figure ci-dessous.

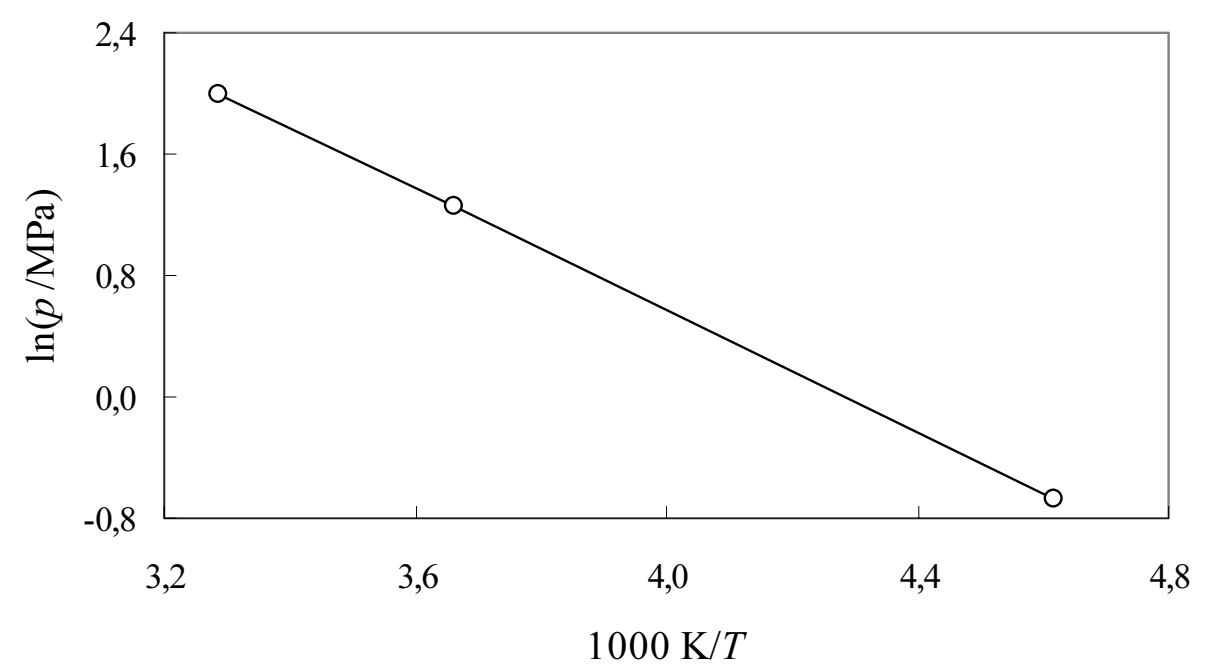


Des formes équivalentes du point de vue algébrique peuvent être utilisées à la place de $10^{3} \mathrm{~K} / T$, comme $\mathrm{kK} / T$, ou $10^{3}(T / \mathrm{K})^{-1}$.

\subsubsection{Symboles des grandeurs et des unités}

Ainsi :

La différence de potentiel

électrique maximale s'exprime sous la forme : $U_{\max }=1000 \mathrm{~V}$ mais pas $U=1000 \mathrm{~V}_{\max }$. La fraction massique du cuivre de l'échantillon de silicium s'exprime sous la forme : $w(\mathrm{Cu})=1,3 \times 10^{-6}$ mais pas $1,3 \times 10^{-6} \mathrm{w} / \mathrm{w}$.

\subsection{3 Écriture de la valeur d'une grandeur}

La valeur numérique précède toujours l'unité et il y a toujours un espace entre le nombre et l'unité. Ainsi la valeur d'une grandeur étant le produit d'un nombre par une unité, l'espace est considéré comme un signe de multiplication (tout comme l'espace entre les unités). Les seules exceptions à la règle sont les symboles d'unité pour le degré, la minute et la seconde d'angle plan, ${ }^{\circ}$, ', et ", respectivement, pour lesquels il n'y a pas d'espace entre la valeur numérique et le symbole d'unité.

Cette règle signifie que le symbole ${ }^{\circ} \mathrm{C}$ pour le degré Celsius est précédé d'un espace pour exprimer la valeur de la température Celsius $t$.

Même lorsque la valeur d'une grandeur est utilisée comme adjectif, il convient de laisser un espace entre la valeur numérique et le symbole de l'unité. Ce n'est que lorsque l'on écrit le nom de l'unité en toutes lettres que l'on applique les règles grammaticales ordinaires (voir un exemple en anglais page 133).

Dans une expression, on n'utilise qu'une seule unité. Les valeurs des grandeurs temps et angle plan exprimées au moyen d'unités en dehors du SI font exception à cette règle. Toutefois, en ce qui concerne l'angle plan, il est généralement préférable de diviser le degré de manière décimale. Ainsi l'on écrira $22,20^{\circ}$ plutôt que $22^{\circ} 12^{\prime}$, sauf dans les domaines tels que la navigation, la cartographie, l'astronomie et pour la mesure d'angles très petits.

\subsection{4 Écriture des nombres et séparateur décimal}

Le symbole utilisé pour séparer le nombre entier de sa partie décimale est appelé séparateur décimal. D'après la $22^{\mathrm{e}}$ Conférence générale (2003, Résolution 10$)$, « le symbole du séparateur décimal pourra être le point sur la ligne ou la virgule sur la ligne ». Le séparateur décimal choisi sera celui qui est d'usage courant dans le contexte.

Si le nombre se situe entre +1 et -1 , le séparateur décimal est toujours précédé d'un zéro.

$-0,234$

mais pas -,234

D'après la $9^{\mathrm{e}}$ Conférence générale (1948, Résolution 7) et la $22^{\mathrm{e}}$ Conférence générale (2003, Résolution 10), les nombres comportant un grand nombre de chiffres peuvent être partagés en tranches de trois chiffres, séparées par un espace, afin de faciliter la lecture. Ces tranches ne sont jamais séparées par des points, ni par des virgules. Cependant, lorsqu'il n'y a que quatre chiffres avant ou après le séparateur décimal, il est d'usage de ne pas isoler un chiffre par un espace. L'habitude de grouper ainsi les $m=12,3 \mathrm{~g}$ où $m$ est utilisé comme symbole de la grandeur masse, mais $\varphi=30^{\circ} 22^{\prime} 8^{\prime \prime}$ où $\varphi$ est utilisé comme symbole de la grandeur angle plan.

$t=30,2^{\circ} \mathrm{C}$

mais pas $t=30,2^{\circ} \mathrm{C}$ ni $t=30,2^{\circ} \mathrm{C}$

une résistance de $10 \mathrm{k} \Omega$

un film de 35 millimètres de largeur

\section{$l=10,234 \mathrm{~m}$ \\ mais pas \\ $l=10 \mathrm{~m} \mathrm{23,4} \mathrm{cm}$}

43279,16829

mais pas $43.279,168.29$

3279,1683

ou 3279,1683 
chiffres est question de choix personnel; elle n'est pas toujours suivie dans certains domaines spécialisés tels que le dessin industriel, les documents financiers et les scripts qui doivent être lus par ordinateur.

En ce qui concerne les nombres d'un tableau, le format utilisé doit être cohérent dans une même colonne.

\subsubsection{Expression de l'incertitude de mesure associée à la valeur d'une grandeur}

L'incertitude associée à la valeur estimée d'une grandeur doit être évaluée et exprimée en accord avec le Guide pour l'expression de l'incertitude de mesure [ISO, 1995]. L'incertitude-type, c'est-à-dire l'écart-type estimé (correspondant à un facteur d'élargissement $k=1$ ), associée à une grandeur $x$ est désignée par $u(x)$. Un moyen commode de représenter l'incertitude est donné dans l'exemple suivant :

$$
m_{\mathrm{n}}=1,67492728(29) \times 10^{-27} \mathrm{~kg}
$$

où $m_{\mathrm{n}}$ est le symbole de la grandeur (ici la masse du neutron) et le nombre entre parenthèses la valeur numérique de l'incertitude-type composée sur les deux derniers chiffres de la valeur estimée de $m_{\mathrm{n}}$, dans le cas présent :

$$
u\left(m_{\mathrm{n}}\right)=0,00000029 \times 10^{-27} \mathrm{~kg} .
$$

Si l'on utilise un facteur d'élargissement $k$ différent de 1 , il faut le préciser.

\subsubsection{Multiplication ou division des symboles des grandeurs, des valeurs des grandeurs et des nombres}

Pour multiplier ou diviser les symboles des grandeurs, il est possible d'utiliser n'importe laquelle des écritures suivantes : $a b, a b, a \cdot b, a \times b, a / b, \frac{a}{b}, a b^{-1}$.

Lorsque l'on multiplie la valeur des grandeurs, il convient d'utiliser soit un signe de multiplication, $\times$, des parenthèses (ou des crochets), mais pas le point à mi-hauteur (centré). Lorsque l'on multiplie des nombres, il convient d'utiliser uniquement le signe de multiplication, $x$.

Lorsque l'on divise les valeurs des grandeurs au moyen d'une barre oblique, on utilise des parenthèses pour lever toute ambiguïté.

\subsubsection{Valeur des grandeurs sans dimension, ou grandeurs de dimension un}

Comme nous l'avons vu dans la section 2.2.3, l'unité SI cohérente des grandeurs sans dimension, ou grandeurs de dimension un, est le nombre un, symbole 1. Les valeurs de ces grandeurs sont exprimées simplement par des nombres. Le symbole d'unité 1 ou nom d'unité « un » n'est pas mentionné explicitement, et il n'y a pas de symbole particulier ni de nom spécial pour l'unité un, à quelques exceptions près mentionnées ci-dessous. Pour la grandeur angle plan, l'unité un porte le nom spécial radian, symbole rad, et pour la grandeur angle solide, elle porte le nom spécial stéradian, symbole sr. Pour les logarithmes de rapports de grandeurs, les noms spéciaux néper, symbol $\mathrm{Np}$, bel, symbole $\mathrm{B}$, et décibel, symbole $\mathrm{dB}$, sont utilisés (voir 4.1 et tableau 8, p. 38).

Parce que les symboles de préfixes SI ne peuvent pas être attachés au symbole $1 \mathrm{ni}$ au nom d'unité « un », les puissances de 10 sont utilisées pour exprimer les valeurs de grandeurs sans dimension particulièrement grandes ou particulièrement petites.

\section{$n=1,51$}

mais pas $n=1,51 \times 1$ où $n$ est le symbole de la grandeur indice de réfraction. 
Dans les expressions mathématiques, le symbole \% (pourcent), reconnu internationalement, peut être utilisé avec le SI pour représenter le nombre 0,01. Ainsi, il peut être utilisé pour exprimer les valeurs des grandeurs sans dimension. Quand il est utilisé, il convient de mettre un espace entre le nombre et le symbole \%. Lorsque l'on exprime les valeurs des grandeurs sans dimension de cette manière, il est préférable d'utiliser le symbole \% plutôt que le nom « pourcent ».

Dans un texte écrit, le symbole \% signifie en général « un pour cent ».

Les expressions telles que "pourcentage de masse », "pourcentage de volume», " pourcentage de quantité de matière », ne doivent pas être utilisées ; les informations sur la grandeur en question doivent être données par le nom et le symbole de la grandeur.

Lorsque l'on exprime les valeurs de fractions sans dimension (par exemple fraction massique, fraction volumique, incertitude relative etc.), il est parfois utile d'employer le rapport entre deux unités de même nature.

Le terme «ppm », qui signifie $10^{-6}$ en valeur relative, ou $1 \times 10^{-6}$, ou «partie par million, millionième», est aussi utilisé. Les termes «partie par milliard» [billion (États-Unis)/trillion (Royaume-Uni)], et leurs abréviations respectives «ppb» et «ppt», sont aussi utilisés, mais leur signification varie selon la langue, c'est pourquoi il est préférable d'éviter de les employer. (Bien que dans les pays de langue anglaise le terme « billion » corresponde à $10^{9}$, et le terme «trillion» à $10^{12}$, le terme «billion» peut parfois correspondre à $10^{12}$ et «trillion» à $10^{18}$. L'abréviation ppt est aussi parfois comprise comme une partie par millier (ou millième), ce qui est source de confusion supplémentaire.)

Il est important de spécifier quelle est la grandeur (sans dimension) dont on donne la valeur, quand on utilise l'un des termes $\%$, ppm etc.
$x_{\mathrm{B}}=0,0025=0,25 \%$,

où $x_{\mathrm{B}}$ est le symbole de la grandeur fraction de

quantité (fraction molaire) de l'entité B.

Le miroir reflète $95 \%$ des photons incidents.

$\varphi=3,6 \%$

mais pas $\varphi=3,6 \%(V / V)$,

où $\varphi$ est la fraction volumique.

$x_{\mathrm{B}}=2,5 \times 10^{-3}$

$=2,5 \mathrm{mmol} / \mathrm{mol}$

$u_{\mathrm{r}}(U)=0,3 \mu \mathrm{V} / \mathrm{V}$

où $u_{r}(U)$ est l'incertitude

relative de la tension

mesurée $U$. 


\section{Annexe 1. Décisions de la Conférence générale des poids et mesures et du Comité international des poids et mesures}

Cette annexe regroupe les décisions de la Conférence générale et du Comité international qui concernent directement les définitions des unités SI, les préfixes à utiliser avec le SI, ainsi que les conventions relatives à l'écriture des symboles d'unités et des nombres. Il ne s'agit pas d'une liste exhaustive des décisions de la Conférence générale et du Comité international. Pour consulter toutes ces décisions, il faut se référer aux volumes successifs des Comptes rendus de la Conférence générale des poids et mesures (CR) et des Procès-verbaux du Comité international des poids et mesures (PV), et aussi, pour les décisions récentes, à Metrologia.

Le SI n'est pas statique, il suit les progrès de la métrologie, aussi certaines décisions ont-elles été abrogées ou modifiées; d'autres ont été précisées par des adjonctions. Les décisions qui ont fait l'objet d'un changement sont identifiées par un astérisque $\left.{ }^{*}\right)$ et renvoient à une note qui fait référence à la décision qui officialise cette modification.

Le texte original des décisions figure dans une police différente (sans empattement) pour le distinguer du texte principal. Les astérisques et notes ont été ajoutés par le BIPM pour rendre le texte plus compréhensible. Ils ne font pas partie des décisions proprement dites.

Les décisions de la Conférence générale et du Comité international figurent dans cette annexe par ordre chronologique, de 1889 à 2005, afin de préserver la continuité. Cependant, afin de pouvoir identifier facilement les décisions concernant un domaine particulier, nous avons ajouté une table des matières, par sujet, indiquant les réunions pendant lesquelles ces décisions ont été adoptées et donnant les numéros des pages où sont reproduites les publications originelles. 


\section{Table des matières de l'annexe 1}

Décisions concernant l'établissement du SI page

9e CGPM, 1948 : décision d'établir le SI

10 CGPM, 1954 : décision d'adopter les six premières unités de base 57

CIPM 1956 : $\quad$ décision d'adopter le nom Système international d'unités $\quad 58$

$11^{\mathrm{e}}$ CGPM, 1960 : $\quad$ confirme le nom et l'abréviation SI, $\quad 59$

noms des préfixes téra à pico, $\quad 59$

établit les unités supplémentaires rad et sr, $\quad 60$

établit la liste de certaines unités dérivées $\quad 60$

CIPM, 1969 : $\quad$ déclarations concernant les unités de base, supplémentaires, dérivées et cohérentes, et utilisation des préfixes $\quad 65$

$\begin{array}{lll}\text { CIPM, } 2001 & \text { « unités SI » et « unités du SI » }\end{array}$

Décisions concernant les unités de base du SI

Longueur

$1^{\text {re }}$ CGPM, 1889 : $\quad$ sanction du Prototype du mètre 52

$7^{\mathrm{e}}$ CGPM, 1927 : définition du mètre par le Prototype international 53

$11^{\mathrm{e}}$ CGPM, 1960 : $\quad$ redéfinition du mètre au moyen de la radiation du krypton 86

15 $5^{\mathrm{e}}$ CGPM, 1975 : $\quad$ recommande la valeur de la vitesse de la lumière $\quad 67$

$17^{\mathrm{e}} \mathrm{CGPM}, 1983$ : $\quad$ redéfinition du mètre en fonction de la vitesse de la lumière, $\quad 71$ $\begin{array}{ll}\text { mise en pratique de la définition du mètre } & 71\end{array}$

CIPM, 2002 : $\quad$ révision de la mise en pratique de la définition du mètre $\quad 77$

CIPM, 2003 : $\quad$ révision de la liste des radiations recommandées $\quad \mathbf{8 0}$

CIPM, 2005 : $\quad$ révision de la liste des radiations recommandées 82

\section{Masse}

$1^{\text {re }}$ CGPM, 1889 : $\quad$ sanction du Prototype du kilogramme

$3^{\mathrm{e}}$ CGPM, 1901 : déclaration au sujet de la différence entre masse et poids, et valeur conventionnelle de $g_{\mathrm{n}} \quad 53$

CIPM, 1967 : $\quad$ déclaration sur les préfixes du gramme $\quad 62$

$21^{\mathrm{e}}$ CGPM, 1999 : $\quad$ redéfinition éventuelle du kilogramme 76

\section{Temps}

CIPM, 1956 : définition de la seconde comme la fraction de l'année tropique 1900

11 $1^{\mathrm{e}}$ CGPM, 1960 : $\quad$ ratifie la définition de la seconde donnée par le CIPM en $1956 \quad 59$ CIPM, 1964 : déclare que l'étalon à employer est la transition hyperfine du césium 133 
12 CGPM, 1964 : donne pouvoir au CIPM de désigner les étalons de fréquence atomique et moléculaire à employer

$13^{\mathrm{e}}$ CGPM, 1967/68 : définit la seconde au moyen de la transition du césium

CCDS, 1970 : définit le Temps atomique international, TAI

14 CGPM, 1971 : demande au CIPM de définir et d'établir le

15 CGPM, 1975 : $\quad$ sanctionne le Temps universel coordonné, UTC

\section{Unités électriques}

CIPM, 1946 : définition des unités mécaniques et électriques dans le SI

14 ${ }^{\mathrm{e}}$ CGPM, 1971 : $\quad$ adopte le nom siemens, symbole $\mathrm{S}$, pour la conductance électrique $\quad 66$

$18^{\mathrm{e}}$ CGPM, 1987 : ajustement prévu des représentations du volt et de l'ohm

CIPM, 1988 :

effet Josephson

CIPM, 1988 :

effet Hall quantique

CIPM, 2000 :

réalisation de l'ohm au moyen de la valeur de la constante de von Klitzing

\section{Température thermodynamique}

9 CGPM, 1948 : $\quad$ adopte le point triple de l'eau comme point de référence pour la température thermodynamique, adopte le degré Celsius, et fixe le zéro comme étant la température de référence inférieure de 0,01 degré à celle du point triple de l'eau

CIPM, 1948 : $\quad$ adopte le nom degré Celsius pour l'échelle de température Celsius

$10^{\mathrm{e}}$ CGPM, 1954 : définit la température thermodynamique du point triple de l'eau à 273,16 degrés Kelvin exactement, définit l'atmosphère normale $\quad 57$

$13^{\text {e }}$ CGPM, 1967/68 : définit officiellement le kelvin, symbole K

CIPM, 1989 : $\quad$ Échelle internationale de température de 1990, EIT-90

CIPM, 2005 : $\quad$ note ajoutée à la définition du kelvin à propos de la composition isotopique de l'eau

\section{Quantité de matière}

14 CGPM, 1971 : définition de la mole, symbole mol, comme

$\begin{array}{ll}7^{\mathrm{e}} \text { unité de base, et règles d'utilisation } & 67\end{array}$

$21^{\mathrm{e}}$ CGPM, 1999 : $\quad$ adopte le nom spécial katal, kat 
CIPM, 1946 : définition des unités photométriques, bougie nouvelle et lumen nouveau

$13^{\text {e }}$ CGPM, 1967/68 : définit la candela, symbole cd, en fonction du corps noir

$16^{\mathrm{e}}$ CGPM, 1979 : redéfinit la candela à partir d'un rayonnement monochromatique

\section{Décisions concernant les unités SI dérivées et les unités supplémentaires}

\section{Unités SI dérivées}

12 $2^{\mathrm{e}}$ CGPM, 1964 : $\quad$ accepte de continuer à utiliser le curie comme unité en dehors du SI $\quad 62$

13 ${ }^{\text {e }}$ CGPM, 1967/68 : donne des exemples d'unités dérivées $\quad 64$

15 CGPM, 1975 : $\quad$ adopte les noms spéciaux becquerel, Bq, et gray, Gy 68

16 ${ }^{\mathrm{e}}$ CGPM, 1979 : $\quad$ adopte le nom spécial sievert, Sv $\quad 69$

CIPM, 1984 : $\quad$ décide de clarifier les relations entre la dose absorbée (unité SI gray) et l'équivalent de dose (unité SI sievert) 72

CIPM, 2002 : modifie les relations entre la dose absorbée et

l'équivalent de dose

\section{Unités supplémentaires}

CIPM, 1980 : $\quad$ décide d'interpréter les unités supplémentaires comme des unités dérivées sans dimension

20 CGPM, 1995 : décide de supprimer la classe des unités supplémentaires, et confirme l'interprétation du CIPM selon laquelle ce sont des unités dérivées sans dimension

Décisions concernant la terminologie et approbation des unités en usage avec le SI

Préfixes SI

12 $2^{\mathrm{e}}$ CGPM, 1964 : décide d'ajouter femto et atto à la liste des préfixes

19e CGPM, 1991 : décide d'ajouter zetta, zepto, yotta et yocto à la liste des préfixes

\section{Symboles d'unités et nombres}

9e CGPM, 1948 : décide des règles d'écriture des symboles d'unités et des nombres

\section{Noms d'unités}

$13^{\mathrm{e}}$ CGPM, 1967/68 : abroge l'utilisation du micron et de la bougie nouvelle comme unités en usage avec le SI 
$22^{\mathrm{e}}$ CGPM, 2003 : décide d'autoriser l'usage du point ou de la virgule sur la ligne comme séparateur décimal

Unités en usage avec le SI : un exemple, le litre

$3^{\text {e }}$ CGPM, 1901: définit le litre comme le volume d'un $1 \mathrm{~kg}$ d'eau

$11^{\mathrm{e}}$ CGPM, 1960 : demande au CIPM d'étudier la différence

entre le décimètre cube et le litre

CIPM, 1961 : recommande d'exprimer les volumes en unités SI et non en litres

12 ${ }^{\mathrm{e}}$ CGPM, 1964 : $\quad$ abroge la précédente définition du litre et recommande d'utiliser le litre comme nom spécial donné au décimètre cube

16 ${ }^{\mathrm{e}}$ CGPM, 1979: décide, à titre exceptionnel, d'autoriser les deux symboles L et 1 pour le litre 
$1^{\text {re }}$ CGPM, 1889

- Sanction des prototypes internationaux du mètre et du kilogramme (CR, 34-38)*

La Conférence générale, considérant

- le «Compte rendu du Président du Comité international » et le «Rapport du Comité international des poids et mesures ", d'où il résulte que, par les soins communs de la Section française de la Commission internationale du Mètre, et du Comité international des poids et mesures, les déterminations métrologiques fondamentales des prototypes internationaux et nationaux du mètre et du kilogramme ont été exécutées dans toutes les conditions de garantie et de précision que comporte l'état actuel de la science ;

- que les prototypes internationaux et nationaux du mètre et du kilogramme sont formés de platine allié à 10 pour 100 d'iridium, à 0,0001 près ;

- l'identité de longueur du Mètre et l'identité de la masse du Kilogramme internationaux avec la longueur du Mètre et la masse du Kilogramme déposés aux Archives de France ;

- que les équations des Mètres nationaux, par rapport au Mètre international, sont renfermées dans la limite de 0,01 millimètre et que ces équations reposent sur une échelle thermométrique à hydrogène qu'il est toujours possible de reproduire, à cause de la permanence de l'état de ce corps, en se plaçant dans des conditions identiques ;

- que les équations des Kilogrammes nationaux, par rapport au Kilogramme international, sont renfermées dans la limite de 1 milligramme ;

- que le Mètre et le Kilogramme internationaux et que les Mètres et les Kilogrammes nationaux remplissent les conditions exigées par la Convention du Mètre,

\section{sanctionne}

A. En ce qui concerne les prototypes internationaux :

1. Le Prototype du mètre choisi par le Comité international. Ce prototype représentera désormais, à la température de la glace fondante, l'unité métrique de longueur.

2. Le Prototype du kilogramme adopté par le Comité international. Ce prototype sera considéré désormais comme unité de masse.

3. L'échelle thermométrique centigrade à hydrogène par rapport à laquelle les équations des Mètres prototypes ont été établies.

B. En ce qui concerne les prototypes nationaux :

$3^{\mathrm{e}}$ CGPM, 1901

I Déclaration concernant la définition du litre $(C R, 38-39)^{*}$

\section{La Conférence déclare :}

1. L'unité de volume, pour les déterminations de haute précision, est le volume occupé par la masse de 1 kilogramme d'eau pure, à son maximum de densité et sous la pression atmosphérique normale ; ce volume est dénommé « litre ».

2
* La définition du mètre a été abrogée en 1960 par la $11^{\mathrm{e}}$ CGPM (Résolution 6 , voir p. 58).

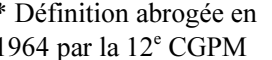

Résolution 6, voir p. 62). 


\section{Déclaration relative à l'unité de masse et à la définition du poids ; valeur conventionnelle de $g_{\mathbf{n}}(\mathrm{CR}, 70)$}

Vu la décision du Comité international des poids et mesures du 15 octobre 1887, par laquelle le kilogramme a été défini comme unité de masse ;

Vu la décision contenue dans la formule de sanction des prototypes du Système métrique, acceptée à l'unanimité par la Conférence générale des poids et mesures dans sa réunion du 26 septembre 1889 ;

Considérant la nécessité de faire cesser l'ambiguïté qui existe encore dans l'usage courant sur la signification du terme poids, employé tantôt dans le sens du terme masse, tantôt dans le sens du terme effort mécanique ;

\section{La Conférence déclare :}

1. Le kilogramme est l'unité de masse ; il est égal à la masse du prototype international du kilogramme ;

2. Le terme poids désigne une grandeur de la même nature qu'une force ; le poids d'un corps est le produit de la masse de ce corps par l'accélération de la pesanteur; en particulier, le poids normal d'un corps est le produit de la masse de ce corps par l'accélération normale de la pesanteur ;

3. Le nombre adopté dans le Service international des Poids et Mesures pour la valeur de l'accélération normale de la pesanteur est $980,665 \mathrm{~cm} / \mathrm{s}^{2}$, nombre sanctionné déjà par quelques législations.

\section{$7^{\mathrm{e}}$ CGPM, 1927}

\section{- Définition du mètre par le Prototype international $(C R, 49)^{*}$}

L'unité de longueur est le mètre, défini par la distance, à $0^{\circ}$, des axes des deux traits médians tracés sur la barre de platine iridié déposée au Bureau international des poids et mesures, et déclarée Prototype du mètre par la Première Conférence générale des poids et mesures, cette règle étant soumise à la pression atmosphérique normale et supportée par deux rouleaux d'au moins un centimètre de diamètre, situés symétriquement dans un même plan horizontal et à la distance de $571 \mathrm{~mm}$ l'un de l'autre.

\section{CIPM, 1946}

】 Définition des unités photométriques ( $P V, 20,119-122)^{*}$

\section{Résolution}

4. Les unités photométriques peuvent être définies comme suit:

Bougie nouvelle (unité d'intensité lumineuse). - La grandeur de la bougie nouvelle est telle que la brillance du radiateur intégral à la température de solidification du platine soit de 60 bougies nouvelles par centimètre carré.

Lumen nouveau (unité de flux lumineux). - Le lumen nouveau est le flux lumineux émis dans l'angle solide unité (stéradian) par une source ponctuelle uniforme ayant une intensité lumineuse de 1 bougie nouvelle.
Cette valeur de $g_{n}$ est la valeur conventionnelle de référence pour le calcul de l'unité kilogramme-force maintenant abolie.
* Définition abrogée en 1960 par la $11^{\mathrm{e}}$ CGPM (Résolution 6, voir p. 58).

\footnotetext{
* Les deux définitions contenues dans cette Résolution furent ratifiées par la $9^{\text {e }}$ CGPM en 1948, qui a en outre approuvé le nom de candela donné à la « bougie nouvelle » (CR, 54). Pour le lumen, le qualificatif « nouveau » a été abandonné par la suite. La définition de la candela a été modifiée par la $13^{\mathrm{e}}$ CGPM en 1967 (Résolution 5, voir p. 64).
}

5. ... 
I Définitions des unités électriques (PV, 20, 132-133)

\section{Résolution 2}

4. (A) Définitions des unités mécaniques utilisées dans les définitions des unités électriques :

Unité de force. - L'unité de force [dans le système MKS (mètre, kilogramme, seconde)] est la force qui communique à une masse de 1 kilogramme l'accélération de 1 mètre par seconde, par seconde.

Joule (unité d'énergie ou de travail). - Le joule est le travail effectué lorsque le point d'application de 1 unité MKS de force [newton] se déplace d'une distance égale à 1 mètre dans la direction de la force.

Watt (unité de puissance). - Le watt est la puissance qui donne lieu à une production d'énergie égale à 1 joule par seconde.

(B) Définitions des unités électriques. Le Comité [international] admet les propositions suivantes définissant la grandeur théorique des unités électriques :

Ampère (unité d'intensité de courant électrique). - L'ampère est l'intensité d'un courant constant qui, maintenu dans deux conducteurs parallèles, rectilignes, de longueur infinie, de section circulaire négligeable et placés à une distance de 1 mètre l'un de l'autre dans le vide, produirait entre ces conducteurs une force égale à $2 \times 10^{-7}$ unité MKS de force [newton] par mètre de longueur.

Volt (unité de différence de potentiel et de force électromotrice). — Le volt est la différence de potentiel électrique qui existe entre deux points d'un fil conducteur transportant un courant constant de 1 ampère, lorsque la puissance dissipée entre ces points est égale à 1 watt.

Ohm (unité de résistance électrique). - L'ohm est la résistance électrique qui existe entre deux points d'un conducteur lorsqu'une différence de potentiel constante de 1 volt, appliquée entre ces deux points, produit, dans ce conducteur, un courant de 1 ampère, ce conducteur n'étant le siège d'aucune force électromotrice.

Coulomb (unité de quantité d'électricité). - Le coulomb est la quantité d'électricité transportée en 1 seconde par un courant de 1 ampère.

Farad (unité de capacité électrique). - Le farad est la capacité d'un condensateur électrique entre les armatures duquel apparaît une différence de potentiel électrique de 1 volt, lorsqu'il est chargé d'une quantité d'électricité égale à 1 coulomb.

Henry (unité d'inductance électrique). - Le henry est l'inductance électrique d'un circuit fermé dans lequel une force électromotrice de 1 volt est produite lorsque le courant électrique qui parcourt le circuit varie uniformément à raison de 1 ampère par seconde.

Weber (unité de flux magnétique). - Le weber est le flux magnétique qui, traversant un circuit d'une seule spire, y produirait une force électromotrice de 1 volt, si on l'amenait à zéro en 1 seconde par décroissance uniforme.

\section{$9^{\mathrm{e}}$ CGPM, 1948}

I Point triple de l'eau ; échelle thermodynamique à un seul point fixe ; unité de quantité de chaleur (joule) (CR, 55 et 63)

\section{Résolution 3}

1. En l'état actuel de la technique, le point triple de l'eau est susceptible de constituer un repère thermométrique avec une précision plus élevée que le point de fusion de la glace.
Les définitions contenues dans cette Résolution ont été approuvées par la $9^{\text {e }}$ CGPM en 1948 (CR, 49), qui a en outre adopté le nom newton (Résolution 7) pour l'unité MKS de force. 
En conséquence, le Comité consultatif [de thermométrie et calorimétrie] estime que le zéro de l'échelle thermodynamique centésimale doit être défini comme étant la température inférieure de 0,0100 degré à celle du point triple de l'eau pure.

2. Le Comité consultatif [de thermométrie et calorimétrie] admet le principe d'une échelle thermodynamique absolue ne comportant qu'un seul point fixe fondamental, constitué actuellement par le point triple de l'eau pure, dont la température absolue sera fixée ultérieurement.

L'introduction de cette nouvelle échelle n'affecte en rien l'usage de l'Échelle internationale, qui reste l'échelle pratique recommandée.

3. L'unité de quantité de chaleur est le joule.

Note: II est demandé que les résultats d'expériences calorimétriques soient autant que possible exprimés en joules. Si les expériences ont été faites par comparaison avec un échauffement d'eau (et que, pour une raison quelconque, on ne puisse éviter l'usage de la calorie), tous les renseignements nécessaires pour la conversion en joules doivent être fournis. Il est laissé aux soins du Comité international, après avis du Comité consultatif de thermométrie et calorimétrie, d'établir une table qui présentera les valeurs les plus précises que l'on peut tirer des expériences faites sur la chaleur spécifique de l'eau, en joules par degré.

Une table, établie conformément à cette demande, a été approuvée et publiée par le Comité international en 1950 (PV, 22, 92).

I Adoption de « degré Celsius » [CIPM, 1948 (PV, 21, 88) et $9^{\mathrm{e}}$ CGPM, 1948 (CR, 64)]

Entre les trois termes (« degré centigrade », « degré centésimal », « degré Celsius ») proposés pour désigner le degré de température, le Comité international a choisi « degré Celsius » (PV, 21, 88).

Ce terme est également adopté par la $9^{e}$ Conférence générale (CR, 64).

\section{Proposition d'établissement d'un système pratique d'unités de mesure (CR, 64)}

\section{Résolution 6}

La Conférence générale,

\section{considérant}

- que le Comité international des poids et mesures a été saisi d'une demande de l'Union internationale de physique le sollicitant d'adopter pour les relations internationales un système pratique international d'unités, recommandant le système MKS et une unité électrique du système pratique absolu, tout en ne recommandant pas que le système CGS soit abandonné par les physiciens ;

- qu'elle-même a reçu du Gouvernement français une demande analogue, accompagnée d'un projet destiné à servir de base de discussion pour l'établissement d'une réglementation complète des unités de mesure ;

charge le Comité international :

- d'ouvrir à cet effet une enquête officielle sur l'opinion des milieux scientifiques, techniques et pédagogiques de tous les pays (en offrant effectivement comme base le document français) et de la pousser activement ;

- de centraliser les réponses ;

- et d'émettre des recommandations concernant l'établissement d'un même système pratique d'unités de mesure, susceptible d'être adopté dans tous les pays signataires de la Convention du Mètre. 


\section{I Écriture des symboles d'unités et des nombres $(C R, 70)^{*}$}

\section{Résolution 7}

\section{Principes}

Les symboles des unités sont exprimés en caractères romains, en général minuscules ; toutefois, si les symboles sont dérivés de noms propres, les caractères romains majuscules sont utilisés. Ces symboles ne sont pas suivis d'un point.

Dans les nombres, la virgule (usage français) ou le point (usage britannique) sont utilisés seulement pour séparer la partie entière des nombres de leur partie décimale. Pour faciliter la lecture, les nombres peuvent être partagés en tranches de trois chiffres : ces tranches ne sont jamais séparées par des points, ni par des virgules.

\begin{tabular}{llll} 
Unités & Symboles & Unités & Symboles \\
\hline - mètre & $\mathrm{m}$ & ampère & $\mathrm{A}$ \\
- mètre carré & $\mathrm{m}^{2}$ & volt & $\mathrm{V}$ \\
- mètre cube & $\mathrm{m}^{3}$ & watt & $\mathrm{W}$ \\
- micron & $\mu$ & ohm & $\Omega$ \\
- litre & $\mathrm{I}$ & coulomb & $\mathrm{C}$ \\
- gramme & $\mathrm{g}$ & farad & $\mathrm{F}$ \\
- tonne & $\mathrm{t}$ & henry & $\mathrm{H}$ \\
seconde & $\mathrm{S}$ & hertz & $\mathrm{Hz}$ \\
erg & $\mathrm{erg}$ & poise & $\mathrm{P}$ \\
dyne & $\mathrm{dyn}$ & newton & $\mathrm{N}$ \\
degré Celsius & ${ }^{\circ} \mathrm{C}$ & c candela (bougie nouvelle) & $\mathrm{cd}$ \\
- degré absolu & ${ }^{\circ} \mathrm{K}$ & lux & $\mathrm{Ix}$ \\
calorie & $\mathrm{cal}$ & lumen & $\mathrm{Im}$ \\
bar & $\mathrm{bar}$ & stilb & $\mathrm{sb}$ \\
heure & $\mathrm{h}$ & &
\end{tabular}

\section{Remarques}

1. Les symboles dont les unités sont précédées d'un point sont ceux qui avaient déjà été antérieurement adoptés par une décision du Comité international.

2. L'unité de volume stère, employée dans le mesurage des bois, aura pour symbole "st» et non plus «s », qui lui avait été précédemment affecté par le Comité international.

3. S'il s'agit, non d'une température, mais d'un intervalle ou d'une différence de température, le mot «degré » doit être écrit en toutes lettres ou par l'abréviation « deg ».
* La Conférence générale a abrogé un certain nombre de décisions concernant les unités et la terminologie, en particulier celles relatives au micron, au degré absolu et aux noms " degré » et « deg », $13^{\mathrm{e}}$ CGPM, 1967/68 (Résolutions 7 et 3 , voir pp. 65 et 63 , respectivement), ainsi qu'au litre, $16^{\mathrm{e}}$ CGPM , 1979 (Résolution 6, voir p. 69). 


\section{Définition de l'échelle thermodynamique de température $(C R, 79)^{*}$}

\section{Résolution 3}

La Dixième Conférence générale des poids et mesures décide de définir l'échelle thermodynamique de température au moyen du point triple de l'eau comme point fixe fondamental, en lui attribuant la température 273,16 degrés Kelvin, exactement.

\section{Définition de l'atmosphère normale $(C R, 79)$}

\section{Résolution 4}

La Dixième Conférence générale des poids et mesures, ayant constaté que la définition de l'atmosphère normale donnée par la Neuvième Conférence générale des poids et mesures dans la définition de l'Échelle internationale de température a laissé penser à quelques physiciens que la validité de cette définition de l'atmosphère normale était limitée aux besoins de la thermométrie de précision,

déclare qu'elle adopte, pour tous les usages, la définition :

1 atmosphère normale $=1013250$ dynes par centimètre carré,

c'est-à-dire : 101325 newtons par mètre carré.

I Système pratique d'unités de mesure $(C R, 80)^{*}$

\section{Résolution 6}

La Dixième Conférence générale des poids et mesures, en exécution du voeu exprimé dans sa Résolution 6 par la Neuvième Conférence générale concernant l'établissement d'un système pratique d'unités de mesure pour les relations internationales,

décide d'adopter comme unités de base de ce système à établir, les unités suivantes:

$\begin{array}{ll}\text { longueur } & \text { mètre } \\ \text { masse } & \text { kilogramme } \\ \text { temps } & \text { seconde } \\ \text { intensité de courant électrique } & \text { ampère } \\ \text { température thermodynamique } & \text { degré Kelvin } \\ \text { intensité lumineuse } & \text { candela }\end{array}$

\section{CIPM, 1956}

I Définition de l'unité de temps (seconde) (PV, 25, 77)*

\section{Résolution 1}

En vertu des pouvoirs que lui a conférés la Dixième Conférence générale des poids et mesures par sa Résolution 5, le Comité international des poids et mesures,

\section{considérant}

1. que la Neuvième Assemblée générale de l'Union astronomique internationale (Dublin, 1955) a émis un avis favorable au rattachement de la seconde à l'année tropique,

2. que, selon les décisions de la Huitième Assemblée générale de l'Union astronomique internationale (Rome, 1952), la seconde de temps des éphémérides (T.E.) est la fraction

\author{
* La $13^{\mathrm{e}}$ CGPM en \\ 1967/68 (Résolution 4, \\ voir p. 64) a explicitement \\ défini le kelvin.
}

* Le nom de l'unité de température thermodynamique a été changé en « kelvin » en 1967 par la $13^{\mathrm{e}}$ CGPM

(Résolution 3, voir p. 63).

* Définition abrogée en 1967 par la $13^{\mathrm{e}} \mathrm{CGPM}$ (Résolution 1, voir p. 63). 
$\frac{12960276813}{408986496} \times 10^{-9}$ de l'année tropique pour 1900 janvier 0 à $12 \mathrm{~h}$ T.E.,

décide

« La seconde est la fraction 1/31 556 925,9747 de l'année tropique pour 1900 janvier 0 à

12 heures de temps des éphémérides. »

I Système international d'unités (PV, 25, 83)

Résolution 3

Le Comité international des poids et mesures,

considérant

- la mission dont l'a chargé la Neuvième Conférence générale des poids et mesures par sa Résolution 6 concernant l'établissement d'un système pratique d'unités de mesure susceptible d'être adopté par tous les pays signataires de la Convention du Mètre,

- l'ensemble des documents envoyés par les vingt et un pays qui ont répondu à l'enquête prescrite par la Neuvième Conférence générale des poids et mesures,

- la Résolution 6 de la Dixième Conférence générale des poids et mesures fixant le choix des unités de base du système à établir,

recommande

1. que soit désigné comme "Système international d'unités » le système fondé sur les unités de base adoptées par la Dixième Conférence générale, qui sont :

[Suit la liste des six unités de base avec leur symbole, reproduite dans la Résolution 12 de la $11^{\mathrm{e}}$ CGPM (1960)].

2. que soient employées les unités de ce système énumérées au tableau suivant, sans préjudice d'autres unités qu'on pourrait ajouter à l'avenir :

[Suit le tableau des unités reproduit dans le paragraphe 4 de la Résolution 12 de la $11^{\mathrm{e}}$ CGPM (1960)].

\section{$11^{\mathrm{e}}$ CGPM, 1960}

Définition du mètre $(C R, 85)^{*}$

\section{Résolution 6}

* Définition abrogée en 1983 par la $17^{\mathrm{e}}$ CGPM (Résolution 1, voir p. 71).

La Onzième Conférence générale des poids et mesures,

\section{considérant}

- que le Prototype international ne définit pas le mètre avec une précision suffisante pour les besoins actuels de la métrologie,

- qu'il est d'autre part désirable d'adopter un étalon naturel et indestructible,

décide

1. Le mètre est la longueur égale à 1650763,73 longueurs d'onde dans le vide de la radiation correspondant à la transition entre les niveaux $2 p_{10}$ et $5 d_{5}$ de l'atome de krypton 86.

2. La définition du mètre en vigueur depuis 1889 , fondée sur le Prototype international en platine iridié, est abrogée.

3. Le Prototype international du mètre sanctionné par la Première Conférence générale des poids et mesures en 1889 sera conservé au Bureau international des poids et mesures dans les mêmes conditions que celles qui ont été fixées en 1889. 
IDéfinition de l'unité de temps (seconde) $(C R, 86)^{*}$

\section{Résolution 9}

La Onzième Conférence générale des poids et mesures,

\section{considérant}

- le pouvoir donné par la Dixième Conférence générale des poids et mesures au Comité international des poids et mesures de prendre une décision au sujet de la définition de l'unité fondamentale de temps,

- la décision prise par le Comité international des poids et mesures dans sa session de 1956,

ratifie la définition suivante:

« La seconde est la fraction 1/31 556925,9747 de l'année tropique pour 1900 janvier 0 à 12 heures de temps des éphémérides. »

\section{Système international d'unités $(\mathrm{CR}, 87)^{*}$}

\section{Résolution 12}

La Onzième Conférence générale des poids et mesures,

\section{considérant}

- la Résolution 6 de la Dixième Conférence générale des poids et mesures par laquelle elle a adopté les six unités devant servir de base à l'établissement d'un système pratique de mesure pour les relations internationales

$\begin{array}{lll}\text { longueur } & \text { mètre } & \mathrm{m} \\ \text { masse } & \text { kilogramme } & \mathrm{kg} \\ \text { temps } & \text { seconde } & \mathrm{s} \\ \text { intensité de courant électrique } & \text { ampère } & \mathrm{A} \\ \text { température thermodynamique } & \text { degré Kelvin } & { }^{\circ} \mathrm{K} \\ \text { intensité lumineuse } & \text { candela } & \mathrm{cd}\end{array}$

- la Résolution 3 adoptée par le Comité international des poids et mesures en 1956,

- les recommandations adoptées par le Comité international des poids et mesures en 1958 concernant l'abréviation du nom de ce système et les préfixes pour la formation des multiples et sous-multiples des unités,

\section{décide}

1. le système fondé sur les six unités de base ci-dessus est désigné sous le nom de « Système international d'unités »;

2. l'abréviation internationale du nom de ce Système est : SI ;

3. les noms des multiples et sous-multiples des unités sont formés au moyen des préfixes suivants :

\begin{tabular}{|c|c|c|c|c|c|}
\hline $\begin{array}{l}\text { Facteur par lequel } \\
\text { l'unité est multipliée }\end{array}$ & Préfixe & Symbole & $\begin{array}{l}\text { Facteur par lequel } \\
\text { l'unité est multipliée }\end{array}$ & $\begin{array}{r}\text { Préfixe } \\
\text { Sy }\end{array}$ & ibole \\
\hline $1000000000000=10^{12}$ & téra & $\mathrm{T}$ & $0,1=10^{-1}$ & déci & $d$ \\
\hline $1000000000=10^{9}$ & giga & G & $0,01=10^{-2}$ & centi & c \\
\hline $1000000=10^{6}$ & méga & $M$ & $0,001=10^{-3}$ & milli & $\mathrm{m}$ \\
\hline $1000=10^{3}$ & kilo & $\mathrm{k}$ & $0,000001=10^{-6}$ & micro & $\mu$ \\
\hline $100=10^{2}$ & hecto & $\mathrm{h}$ & $0,000000001=10^{-9}$ & nano & $\mathrm{n}$ \\
\hline $10=10^{1}$ & déca & da & $0,000000000001=10^{-12}$ & pico & $\mathrm{p}$ \\
\hline
\end{tabular}

* Définition abrogée en 1967 par la $13^{\mathrm{e}}$ CGPM (Résolution 1, voir p. 63).
* La Conférence générale a ultérieurement abrogé certaines de ces décisions et complété la liste des préfixes SI : voir notes cidessous.
Le nom et symbole de l'unité de température thermodynamique ont été modifiés par la $13^{\mathrm{e}} \mathrm{CGPM}$ en 1967 (Résolution 3, voir $\mathrm{p} .63)$.

Une septième unité de base, la mole, fut adoptée par la $14^{\mathrm{e}} \mathrm{CGPM}$ en 1971 (Résolution 3, voir p. 67).

D'autres préfixes furent adoptés par la $12^{\mathrm{e}} \mathrm{CGPM}$ en 1964 (Résolution 8, voir p. 62), par la $15^{\mathrm{e}} \mathrm{CGPM}$ en 1975 (Résolution 10, voir p. 68) et par la $19^{\mathrm{e}} \mathrm{CGPM}$ en 1991 (Résolution 4 voir p. 75) 
4. sont employées dans ce Système les unités ci-dessous, sans préjudice d'autres unités qu'on pourrait ajouter à l'avenir

\section{Unités supplémentaires}

angle

angle solide

\section{Unités dérivées}

superficie
volume
fréquence
masse volumique (densité)
vitesse
vitesse angulaire
accélération
accélération angulaire
force
pression (tension mécanique)
viscosité cinématique
viscosité dynamique

travail, énergie, quantité de

chaleur

puissance

quantité d'électricité

tension électrique, différence de

potentiel, force électromotrice

intensité de champ électrique

résistance électrique

capacité électrique

flux d'induction magnétique

inductance

induction magnétique

intensité de champ magnétique

force magnétomotrice

flux lumineux

luminance

éclairement radian rad

stéradian

$\mathrm{sr}$

mètre carré $\quad \mathrm{m}^{2}$

mètre cube $\mathrm{m}^{3}$

hertz $\mathrm{Hz}$

kilogramme par mètre cube $\mathrm{kg} / \mathrm{m}^{3}$

mètre par seconde

radian par seconde

$\mathrm{m} / \mathrm{s}$

mètre par seconde carrée

radian par seconde carrée

newton

newton par mètre carré

mètre carré par seconde

newton-seconde

par mètre carré

joule

watt

coulomb

volt

$m^{2}$
$m^{3}$
$H z$

$\mathrm{rad} / \mathrm{s}$

$\mathrm{m} / \mathrm{s}^{2}$

$\mathrm{rad} / \mathrm{s}^{2}$

$\mathrm{N} \quad \mathrm{kg} \cdot \mathrm{m} / \mathrm{s}^{2}$

$\mathrm{N} / \mathrm{m}^{2}$

$\mathrm{m}^{2} / \mathrm{s}$

$\mathrm{N} \cdot \mathrm{s} / \mathrm{m}^{2}$

$J$

$\mathrm{N} \cdot \mathrm{m}$

volt par mètre

ohm

farad

weber

henry

tesla

ampère par mètre

ampère

lumen

candela par mètre carré

lux
La $20^{\circ}$ CGPM a abrogé en 1995 la classe des unités supplémentaires dans le SI (Résolution 8, voir p. 75). Ces unités sont maintenant considérées comme des unités dérivées.

La $13^{\mathrm{e}}$ CGPM en 1967

(Résolution 6, voir p. 64) a ajouté d'autres unités à cette liste d'unités dérivées, qui, en principe, n'est pas limitative.

\section{Décimètre cube et litre $(C R, 88)$}

\section{Résolution 13}

La Onzième Conférence générale des poids et mesures,

\section{considérant}

- que le décimètre cube et le litre sont inégaux et diffèrent d'environ 28 millionièmes, 
- que les déterminations de grandeurs physiques impliquant des mesures de volume ont une précision de plus en plus élevée, aggravant par là les conséquences d'une confusion possible entre le décimètre cube et le litre,

invite le Comité international des poids et mesures à mettre ce problème à l'étude et à présenter ses conclusions à la Douzième Conférence générale.

\section{CIPM, 1961}

I Décimètre cube et litre $(P V, 29,34)$

\section{Recommandation}

Le Comité international des poids et mesures recommande que les résultats des mesures précises de volume soient exprimés en unités du Système international et non en litres.

\section{CIPM, 1964}

I Étalons de fréquence (PV, 32, 26 et $C R, 93)$

\section{Déclaration}

Le Comité international des poids et mesures,

habilité par la Résolution 5 de la Douzième Conférence générale des poids et mesures à désigner les étalons atomiques ou moléculaires de fréquence à employer temporairement pour les mesures physiques de temps,

déclare que l'étalon à employer est la transition entre les niveaux hyperfins $F=4, M=0$ et $F=3, M=0$ de l'état fondamental ${ }^{2} S_{1 / 2}$ de l'atome de césium 133 non perturbé par des champs extérieurs, et que la valeur 9192631770 hertz est assignée à la fréquence de cette transition.

\section{$12^{\mathrm{e}}$ CGPM, 1964}

\section{I Étalon atomique de fréquence $(C R, 93)$}

\section{Résolution 5}

La Douzième Conférence générale des poids et mesures,

\section{considérant}

- que la Onzième Conférence générale des poids et mesures a constaté dans sa Résolution 10 l'urgence pour les buts de la haute métrologie d'arriver à un étalon atomique ou moléculaire d'intervalle de temps,

- que, malgré les résultats acquis dans l'utilisation des étalons atomiques de fréquence à césium, le moment n'est pas encore venu pour la Conférence générale d'adopter une nouvelle définition de la seconde, unité de base du Système international d'unités, en raison des progrès nouveaux et importants qui peuvent être obtenus à la suite des études en cours,

considérant aussi qu'on ne peut pas attendre davantage pour fonder les mesures physiques de temps sur des étalons atomiques ou moléculaires de fréquence,

habilite le Comité international des poids et mesures à désigner les étalons atomiques ou moléculaires de fréquence à employer temporairement,

invite les Organisations et les Laboratoires experts dans ce domaine à poursuivre les études utiles à une nouvelle définition de la seconde. 


\section{Litre $(C R, 93)$}

\section{Résolution 6}

La Douzième Conférence générale des poids et mesures,

considérant la Résolution 13 adoptée par la Onzième Conférence générale en 1960 et la Recommandation adoptée par le Comité international des poids et mesures à sa session de 1961,

1. abroge la définition du litre donnée en 1901 par la Troisième Conférence générale des poids et mesures,

2. déclare que le mot « litre » peut être utilisé comme un nom spécial donné au décimètre cube,

3. recommande que le nom de litre ne soit pas utilisé pour exprimer les résultats des mesures de volume de haute précision.

\section{Curie $(C R, 94)^{*}$}

\section{Résolution 7}

La Douzième Conférence générale des poids et mesures,

considérant que depuis longtemps le curie est utilisé dans beaucoup de pays comme unité pour l'activité des radionucléides,

reconnaissant que dans le Système international d'unités $(\mathrm{SI})$, l'unité de cette activité est la seconde à la puissance moins un $\left(\mathrm{s}^{-1}\right)$,

admet que le curie soit encore retenu comme unité en dehors du SI pour l'activité, avec la valeur $3,7 \times 10^{10} \mathrm{~s}^{-1}$. Le symbole de cette unité est $\mathrm{Ci}$.

\section{Préfixes SI femto et atto $(\mathrm{CR}, 94)^{*}$}

\section{Résolution 8}

La Douzième Conférence générale des poids et mesures,

décide d'ajouter à la liste des préfixes pour la formation des noms des multiples et des sous-multiples des unités, adoptée par la Onzième Conférence générale, Résolution 12, paragraphe 3 , les deux nouveaux préfixes suivants :

Facteur par lequel

l'unité est multipliée

Préfixe

Symbole

\begin{tabular}{lll}
\hline $10^{-15}$ & femto & $\mathrm{f}$ \\
$10^{-18}$ & atto & $\mathrm{a}$
\end{tabular}

\section{CIPM, 1967}

I Multiples et sous-multiples décimaux de l'unité de masse (PV, 35, 29 et Metrologia, 1968, 4, 45)

\section{Recommandation 2}

Le Comité international des poids et mesures,

considérant que la règle de formation des noms des multiples et sous-multiples décimaux des unités du paragraphe 3 de la Résolution 12 de la Onzième Conférence générale des poids et mesures (1960) peut prêter à des interprétations divergentes dans son application à l'unité de masse,

\section{* Le nom « becquerel» (Bq) a été adopté par la $15^{\mathrm{e}} \mathrm{CGPM}$ en 1975 (Résolution 8, voir p. 68) pour l'unité SI d'activité : $1 \mathrm{Ci}=3,7 \times 10^{10} \mathrm{~Bq}$.}

* De nouveaux préfixes furent ajoutés par la $15^{\mathrm{e}}$ CGPM en 1975 (Résolution 10, voir p. 68). 
déclare que les dispositions de la Résolution 12 de la Onzième Conférence générale s'appliquent dans le cas du kilogramme de la façon suivante : les noms des multiples et sous-multiples décimaux de l'unité de masse sont formés par l'adjonction des préfixes au mot « gramme ».

\section{$13^{\mathrm{e}}$ CGPM, $1967 / 68$}

I Unité SI de temps (seconde) (CR, 103 et Metrologia, 1968, 4, 43)

\section{Résolution 1}

La Treizième Conférence générale des poids et mesures, considérant

- que la définition de la seconde décidée par le Comité international des poids et mesures à sa session de 1956 (Résolution 1) et ratifiée par la Résolution 9 de la Onzième Conférence générale (1960), puis maintenue par la Résolution 5 de la Douzième Conférence générale (1964) ne suffit pas aux besoins actuels de la métrologie,

- qu'à sa session de 1964 le Comité international des poids et mesures, habilité par la Résolution 5 de la Douzième Conférence (1964), a désigné pour répondre à ces besoins un étalon atomique de fréquence à césium à employer temporairement,

- que cet étalon de fréquence est maintenant suffisamment éprouvé et suffisamment précis pour servir à une définition de la seconde répondant aux besoins actuels,

- que le moment est venu de remplacer la définition actuellement en vigueur de l'unité de temps du Système international d'unités par une définition atomique fondée sur cet étalon,

\section{décide}

1. L'unité de temps du Système international d'unités est la seconde définie dans les termes suivants :

« La seconde est la durée de 9192631770 périodes de la radiation correspondant à la transition entre les deux niveaux hyperfins de l'état fondamental de l'atome de césium $133 »$.

2. La Résolution 1 adoptée par le Comité international des poids et mesures à sa session de 1956 et la Résolution 9 de la Onzième Conférence générale des poids et mesures sont abrogées.

I Unité SI de température thermodynamique (kelvin) (CR, 104 et Metrologia, $1968,4,43)^{*}$

\section{Résolution 3}

La Treizième Conférence générale des poids et mesures,

\section{considérant}

- les noms « degré Kelvin » et " degré », les symboles " ${ }^{\circ} \mathrm{K}$ " et « deg » et leurs règles d'emploi contenus dans la Résolution 7 de la Neuvième Conférence générale (1948), dans la Résolution 12 de la Onzième Conférence générale (1960) et la décision prise par le Comité international des poids et mesures en 1962 (PV, 30, 27),

- que l'unité de température thermodynamique et l'unité d'intervalle de température sont une même unité qui devrait être désignée par un nom unique et par un symbole unique,
Lors de sa session de 1997, le Comité international a confirmé que cette définition se réfère à un atome de césium au repos, à une température de $0 \mathrm{~K}$.

* À sa session de 1980, le Comité international a approuvé le rapport de la $7^{\mathrm{e}}$ session du CCU demandant que l'emploi des symboles $4{ }^{\circ} \mathrm{K}$ » et « deg » ne soit plus admis. 


\section{décide}

1. l'unité de température thermodynamique est désignée sous le nom "kelvin » et son symbole est « $\mathrm{K}$ »;*

2. ce même nom et ce même symbole sont utilisés pour exprimer un intervalle de température ;

3. un intervalle de température peut aussi s'exprimer en degrés Celsius ;

4. les décisions mentionnées au premier considérant concernant le nom de l'unité de température thermodynamique, son symbole et la désignation de l'unité pour exprimer un intervalle ou une différence de température sont abrogées, mais les usages qui sont la conséquence de ces décisions restent admis temporairement.

I Définition de l'unité SI de température thermodynamique (kelvin) (CR, 104 et Metrologia, 1968, 4, 43)*

\section{Résolution 4}

La Treizième Conférence générale des poids et mesures,

considérant qu'il est utile de formuler dans une rédaction explicite la définition de l'unité de température thermodynamique contenue dans la Résolution 3 de la Dixième Conférence générale (1954),

décide d'exprimer cette définition de la façon suivante :

«Le kelvin, unité de température thermodynamique, est la fraction 1/273,16 de la température thermodynamique du point triple de l'eau. »

I Unité SI d'intensité lumineuse (candela) (CR, 104 et Metrologia, 1968, 4, $43-44)^{*}$

\section{Résolution 5}

La Treizième Conférence générale des poids et mesures,

\section{considérant}

- la définition de l'unité d'intensité lumineuse ratifiée par la Neuvième Conférence générale (1948) et contenue dans la « Résolution concernant le changement des unités photométriques » adoptée par le Comité international des poids et mesures en 1946 ( $P V, 20,119)$ en vertu des pouvoirs conférés par la Huitième Conférence générale (1933),

- que cette définition fixe bien la grandeur de l'unité d'intensité lumineuse mais prête à des critiques d'ordre rédactionnel,

décide d'exprimer la définition de la candela de la façon suivante :

« La candela est l'intensité lumineuse, dans la direction perpendiculaire, d'une surface de $1 / 600000$ mètre carré d'un corps noir à la température de congélation du platine sous la pression de 101325 newtons par mètre carré. »

I Unités SI dérivées (CR, 105 et Metrologia, 1968, 4, 44)*

\section{Résolution 6}

La Treizième Conférence générale des poids et mesures,

considérant qu'il est utile de citer d'autres unités dérivées dans la liste du paragraphe 4 de la Résolution 12 de la Onzième Conférence générale (1960),
* Voir la

Recommandation 2

(CI-2005) du CIPM

relative à la composition isotopique de l'eau entrant dans la définition du kelvin (p. 81).

* Voir la

Recommandation 5 (CI-1989) du CIPM relative à l'Échelle internationale de température de 1990 (p. 74).

* Définition abrogée en 1979 par la $16^{\mathrm{e}}$ CGPM (Résolution 3, voir p. 68).
* L'unité d'activité a reçu un nom spécial et un symbole particulier lors de la $15^{\mathrm{e}}$ CGPM en 1975 (Résolution 8, voir p. 68). 
décide d'y ajouter:

nombre d'ondes

entropie

chaleur massique

conductivité thermique

intensité énergétique

activité (d'une source radioactive)
1 par mètre

joule par kelvin

joule par kilogramme kelvin

watt par mètre kelvin

watt par stéradian

1 par seconde $\mathrm{m}^{-1}$

$\mathrm{J} / \mathrm{K}$

$\mathrm{J} /(\mathrm{kg} \cdot \mathrm{K})$

$\mathrm{W} /(\mathrm{m} \cdot \mathrm{K})$

$\mathrm{W} / \mathrm{sr}$

$\mathrm{s}^{-1}$

\section{Abrogation de décisions antérieures (micron et bougie nouvelle) $(C R, 105$ et Metrologia, 1968, 4, 44)}

\section{Résolution 7}

La Treizième Conférence générale des poids et mesures,

considérant que les décisions prises ultérieurement par la Conférence générale concernant le Système international d'unités contredisent quelques parties de la Résolution 7 de la Neuvième Conférence générale (1948),

décide en conséquence de retirer de la Résolution 7 de la Neuvième Conférence :

1. le nom d'unité « micron », et le symbole « $\mu$ » qui fut attribué à cette unité et qui est devenu un préfixe ;

2. le nom d'unité « bougie nouvelle ».

\section{CIPM, 1969}

【 Système international d'unités, modalités d'application de la Résolution 12 de la $11^{\mathrm{e}}$ CGPM (1960) (PV, 37, 30 et Metrologia, 1970, 6, 66)*

\section{Recommandation 1}

Le Comité international des poids et mesures,

considérant que la Résolution 12 de la Onzième Conférence générale des poids et mesures (1960), concernant le Système international d'unités, a suscité des discussions sur certaines dénominations,

\section{déclare}

1. les unités de base, les unités supplémentaires et les unités dérivées du Système international d'unités, qui forment un ensemble cohérent, sont désignées sous le nom d'« unités SI »;**

2. les préfixes adoptés par la Conférence générale pour la formation des multiples et sous-multiples décimaux des unités SI sont appelés « préfixes SI »;

\section{et recommande}

3. d'employer les unités SI et leurs multiples et sous-multiples décimaux dont les noms sont formés au moyen des préfixes SI.

Note: L'appellation "unités supplémentaires », figurant dans la Résolution 12 de la Onzième Conférence générale des poids et mesures (et dans la présente Recommandation), est donnée aux unités SI pour lesquelles la Conférence générale ne décide pas s'il s'agit d'unités de base ou bien d'unités dérivées.

\author{
* $\mathrm{La} 20^{\mathrm{e}} \mathrm{CGPM}$ a décidé \\ d'abroger en 1995 \\ (Résolution 8, voir p. 75) \\ la classe des unités \\ supplémentaires dans le SI.
}

** Le CIPM a approuvé en 2001 une proposition du $\mathrm{CCU}$ visant à clarifier la définition des « unités SI » et « unités du SI », voir p. 77. 
CCDS, 1970 (In CIPM, 1970)

I Définition du TAI (PV, 38, 110-111 et Metrologia, 1971, 7, 43)

\section{Recommandation S 2}

Le Temps atomique international est la coordonnée de repérage temporel établie par le Bureau international de l'heure sur la base des indications d'horloges atomiques fonctionnant dans divers établissements conformément à la définition de la seconde, unité de temps du Système international d'unités.

En 1980, la définition du TAI a été complétée comme suit (déclaration du CCDS, BIPM Com. cons. déf. seconde, 1980, 9, S 15 et Metrologia, 1981, 17, 70) :

Le TAl est une échelle de temps-coordonnée définie dans un repère de référence géocentrique avec comme unité d'échelle la seconde du SI telle qu'elle est réalisée sur le géoïde en rotation.

\section{$14^{\mathrm{e}}$ CGPM, 1971}

\section{Pascal et siemens $(\mathrm{CR}, 78)$}

La $14^{\mathrm{e}}$ Conférence générale a adopté les noms spéciaux «pascal 》 (symbole Pa) pour l'unité SI newton par mètre carré et "siemens" (symbole S) pour l'unité SI de conductance électrique (ohm à la puissance moins un).

I Temps atomique international ; rôle du CIPM (CR, 77-78 et Metrologia, 1972, 8, 35)

\section{Résolution 1}

La Quatorzième Conférence générale des poids et mesures,

\section{considérant}

- que la seconde, unité de temps du Système international d'unités, est définie depuis 1967 d'après une fréquence atomique naturelle, et non plus d'après des échelles de temps fournies par des mouvements astronomiques,

- que le besoin d'une échelle de Temps atomique international (TAl) est une conséquence de la définition atomique de la seconde,

- que plusieurs organisations internationales ont assuré et assurent encore avec succès l'établissement des échelles de temps fondées sur des mouvements astronomiques, particulièrement grâce aux services permanents du Bureau international de l'heure (BIH),

- que le Bureau international de l'heure a commencé à établir une échelle de temps atomique dont les qualités sont reconnues et qui a prouvé son utilité,

- que les étalons atomiques de fréquence servant à la réalisation de la seconde ont été considérés et doivent continuer de l'être par le Comité international des poids et mesures assisté d'un Comité consultatif, et que l'intervalle unitaire de l'échelle de Temps atomique international doit être la seconde réalisée conformément à sa définition atomique,

- que toutes les organisations scientifiques internationales compétentes et les laboratoires nationaux actifs dans ce domaine ont exprimé le désir que le Comité international et la Conférence générale des poids et mesures donnent une définition du Temps atomique international, et contribuent à l'établissement de l'échelle de Temps atomique international,

- que l'utilité du Temps atomique international nécessite une coordination étroite avec les échelles de temps fondées sur des mouvements astronomiques,
L'Union astronomique internationale a précisé cette définition dans sa Résolution A4 de 1991 : « Le TAI est une échelle de temps réalisée dont la forme idéale, si l'on néglige un décalage constant de 32,184 s, est le Temps terrestre (TT), luimême relié à la coordonnée temps du référentiel géocentrique, le Tempscoordonnée géocentrique (TCG), par une marche constante. »

(voir Proc. 21st General Assembly of the IAU, IAU Trans., 1991, vol. XXIB, Kluwer.) 
demande au Comité international des poids et mesures

1. de donner une définition du Temps atomique international ;

2. de prendre les mesures nécessaires, en accord avec les organisations internationales intéressées, pour que les compétences scientifiques et les moyens d'action existants soient utilisés au mieux pour la réalisation de l'échelle de Temps atomique international, et pour que soient satisfaits les besoins des utilisateurs du Temps atomique international.

I Unité SI de quantité de matière (mole) (CR, 78 et Metrologia, 1972, 8, 36)*

\section{Résolution 3}

La Quatorzième Conférence générale des poids et mesures,

considérant les avis de l'Union internationale de physique pure et appliquée, de l'Union internationale de chimie pure et appliquée et de l'Organisation internationale de normalisation concernant le besoin de définir une unité de quantité de matière,

\section{décide}

1. La mole est la quantité de matière d'un système contenant autant d'entités élémentaires qu'il y a d'atomes dans 0,012 kilogramme de carbone 12 ; son symbole est « $\mathrm{mol} »$.

2. Lorsqu'on emploie la mole, les entités élémentaires doivent être spécifiées et peuvent être des atomes, des molécules, des ions, des électrons, d'autres particules ou des groupements spécifiés de telles particules.

3. La mole est une unité de base du Système international d'unités.

\section{$15^{\mathrm{e}}$ CGPM, 1975}

I Valeur recommandée pour la vitesse de la lumière (CR, 103 et Metrologia, 1975, 11, 179-180)

\section{Résolution 2}

La Quinzième Conférence générale des poids et mesures,

considérant l'excellent accord entre les résultats des mesures de longueur d'onde portant sur des radiations de lasers asservis sur une raie d'absorption moléculaire dans la région visible ou infrarouge, avec une incertitude estimée à $\pm 4 \times 10^{-9}$ qui correspond à l'indétermination de la réalisation du mètre,

considérant aussi les mesures concordantes de la fréquence de plusieurs de ces radiations,

recommande l'emploi de la valeur qui en résulte pour la vitesse de propagation des ondes électromagnétiques dans le vide $c=299792458$ mètres par seconde.

\section{Temps universel coordonné (UTC) (CR, 104 et Metrologia, 1975, 11, 180)}

\section{Résolution 5}

La Quinzième Conférence générale des poids et mesures,

considérant que le système appelé «Temps universel coordonné » (UTC) est employé très largement, qu'il est diffusé par la plupart des émetteurs hertziens de signaux horaires, que sa diffusion fournit aux utilisateurs à la fois des fréquences étalons, le Temps atomique international et une approximation du Temps universel (ou, si l'on préfère, du temps solaire moyen),
La définition du TAI a été donnée par le CCDS en 1970 (maintenant renommé CCTF), voir p. 66.

* À sa session de 1980, le CIPM a approuvé le rapport de la $7^{\mathrm{e}}$ session du CCU (1980) précisant que, dans cette définition, il est entendu que l'on se réfère à des atomes de carbone 12 non liés, au repos et dans leur état fondamental.

L'incertitude relative doit être comprise comme étant trois fois l'incertitude-type estimée sur les résultats considérés. 
constate que ce Temps universel coordonné est à la base du temps civil dont l'usage est légal dans la plupart des pays,

estime que cet emploi est parfaitement recommandable.

I Unités SI pour les rayonnements ionisants (becquerel et gray) (CR, 105 et Metrologia, 1975, 11, 180)*

\section{Résolutions 8 et 9}

La Quinzième Conférence générale des poids et mesures,

en raison de l'urgence, exprimée par la Commission internationale des unités et mesures de rayonnements (ICRU), d'étendre l'usage du Système international d'unités aux recherches et aux applications de la radiologie,

en raison de la nécessité de rendre aussi simple que possible l'usage des unités aux nonspécialistes,

tenant compte aussi de la gravité des risques d'erreurs dans la thérapeutique,

adopte le nom spécial suivant d'unité SI pour l'activité :

le becquerel, symbole $\mathrm{Bq}$, égal à la seconde à la puissance moins un (Résolution 8),

adopte le nom spécial suivant d'unité SI pour les rayonnements ionisants :

le gray, symbole Gy, égal au joule par kilogramme (Résolution 9).

Note: Le gray est l'unité SI de dose absorbée. Dans le domaine des rayonnements ionisants, le gray peut encore être employé avec d'autres grandeurs physiques qui s'expriment aussi en joules par kilogramme; le Comité consultatif des unités est chargé d'étudier cette question en collaboration avec les organisations internationales compétentes.

I Préfixes SI péta et exa (CR, 106 et Metrologia, 1975, 11, 180-181)*

\section{Résolution 10}

La Quinzième Conférence générale des poids et mesures,

décide d'ajouter à la liste des préfixes SI pour la formation des noms des multiples des unités, adoptée par la Onzième Conférence générale, Résolution 12, paragraphe 3, les deux préfixes suivants :

Facteur par lequel

l'unité est multipliée

$10^{15} \quad$ peta $P$

$10^{18}$

exa

E

\footnotetext{
* De nouveaux préfixes furent ajoutés en 1991 par la $19^{\mathrm{e}} \mathrm{CGPM}$

(Résolution 4, voir p. 75).

En français, il est d'usage courant de mettre un accent à péta.
}

\section{$16^{\mathrm{e}}$ CGPM, 1979}

I Unité SI d'intensité lumineuse (candela) (CR, 100 et Metrologia, 1980, 16, 56)

\section{Résolution 3}

La Seizième Conférence générale des poids et mesures,

\section{considérant}

- que, malgré les efforts méritoires de quelques laboratoires, il subsiste des divergences excessives entre les résultats de la réalisation de la candela à l'aide du corps noir étalon primaire actuel, 
- que les techniques radiométriques se développent rapidement, autorisant des précisions qui sont déjà analogues à celles de la photométrie et que ces techniques sont déjà en usage dans des laboratoires nationaux pour réaliser la candela sans avoir à construire un corps noir,

- que la relation entre les grandeurs lumineuses de la photométrie et les grandeurs énergétiques, à savoir la valeur 683 lumens par watt pour l'efficacité lumineuse spectrale de la radiation monochromatique de fréquence $540 \times 10^{12}$ hertz, a été adoptée par le Comité international des poids et mesures en 1977,

- que cette valeur a été reconnue suffisamment exacte pour le système des grandeurs lumineuses photopiques, qu'elle n'entraîne qu'un changement d'environ $3 \%$ pour le système des grandeurs lumineuses scotopiques et que par conséquent elle assure une continuité satisfaisante,

- que le moment est venu de donner à la candela une définition susceptible d'améliorer la facilité d'établissement des étalons photométriques et leur précision, et qui s'applique aux grandeurs photopiques et scotopiques de la photométrie et aux grandeurs à définir dans le domaine mésopique,

\section{décide}

1. La candela est l'intensité lumineuse, dans une direction donnée, d'une source qui émet un rayonnement monochromatique de fréquence $540 \times 10^{12}$ hertz et dont l'intensité énergétique dans cette direction est $1 / 683$ watt par stéradian.

2. La définition de la candela (à l'époque appelée bougie nouvelle) décidée par le Comité international des poids et mesures en 1946 en vertu des pouvoirs conférés par la $8^{\mathrm{e}}$ Conférence générale des poids et mesures (CGPM) en 1933, ratifiée par la $9^{e}$ CGPM en 1948, puis amendée par la $13^{\mathrm{e}}$ CGPM en 1967, est abrogée.

I Nom spécial pour l'unité SI d'équivalent de dose (sievert) $(C R, 100$ et Metrologia, 1980, 16, 56)*

\section{Résolution 5}

La Seizième Conférence générale des poids et mesures,

\section{considérant}

- l'effort fait pour introduire les unités SI dans le domaine des rayonnements ionisants,

- les risques que peuvent encourir des êtres humains soumis à des irradiations sousestimées, risques qui pourraient résulter de la confusion entre dose absorbée et équivalent de dose,

- que la prolifération des noms spéciaux représente un danger pour le Système international d'unités et doit être évitée dans toute la mesure du possible, mais que cette règle peut être transgressée lorsqu'il s'agit de sauvegarder la santé humaine,

adopte le nom spécial sievert, symbole Sv, pour l'unité SI d'équivalent de dose dans le domaine de la radioprotection. Le sievert est égal au joule par kilogramme.

I Symboles du litre (CR, 101 et Metrologia, 1980, 16, 56-57)

\section{Résolution 6}

La Seizième Conférence générale des poids et mesures,

reconnaissant les principes généraux adoptés pour l'écriture des symboles des unités dans la Résolution 7 de la $9^{\mathrm{e}}$ Conférence générale des poids et mesures (1948),

considérant que le symbole I pour l'unité litre a été adopté par le Comité international des poids et mesures en 1879 et confirmé dans cette même Résolution de 1948,
La vision photopique est détectée sur la rétine de l'œil par les cônes, sensibles aux luminances élevées $(L>$ environ $10 \mathrm{~cd} \mathrm{~m}^{-2}$ ), qui correspondent à la vision diurne. La vision scotopique est détectée sur la rétine de l'œil par les bâtonnets, sensibles aux faibles luminances $\left(L<\right.$ environ $10^{-3} \mathrm{~cd} \mathrm{~m}^{-2}$ ), qui correspondent à la vision nocturne. Dans le cas de luminances intermédiaires entre la vision photopique et la vision scotopique, les cônes et les bâtonnets fonctionnent simultanément, on est alors dans le domaine de la vision mésopique.

* Le Comité international a décidé en 1984 d'accompagner cette Résolution d'une explication, (Recommandation 1, voir p. 72). 
considérant aussi que, afin d'éviter un risque de confusion entre la lettre I et le chiffre 1 , plusieurs pays ont adopté le symbole $L$ au lieu de I pour l'unité litre,

considérant que le nom litre, bien qu'il ne soit pas inclus dans le Système international d'unités, doit être admis pour l'usage général avec ce Système,

décide, à titre exceptionnel, d'adopter les deux symboles I et $\mathrm{L}$ comme symboles utilisables pour l'unité litre,

considérant en outre que dans l'avenir un seul des deux symboles devrait être retenu,

invite le Comité international des poids et mesures à suivre le développement de l'usage des deux symboles et à donner à la $18^{\mathrm{e}}$ Conférence générale des poids et mesures son avis sur la possibilité de supprimer l'un d'eux.

\section{CIPM, 1980}

I Unités SI supplémentaires (radian et stéradian) (PV, 48, 24 et Metrologia, $1981,17,72)^{*}$

\section{Recommandation 1}

Le Comité international des poids et mesures (CIPM),

prenant en considération la Résolution 3 adoptée par I'ISO/TC 12 en 1978 et la Recommandation U 1 (1980) adoptée par le Comité consultatif des unités (CCU) à sa $7^{\mathrm{e}}$ session

\section{considérant}

- que les unités radian et stéradian sont introduites usuellement dans des expressions des unités pour des besoins de clarification, notamment en photométrie où le stéradian joue un rôle important pour distinguer les unités correspondant aux diverses grandeurs,

- que dans les équations utilisées on exprime généralement l'angle plan comme le rapport entre deux longueurs et l'angle solide comme le rapport entre une aire et le carré d'une longueur, et que par conséquent ces grandeurs sont traitées comme des grandeurs sans dimension,

- que l'étude des formalismes en usage dans le domaine scientifique montre qu'il n'en existe aucun qui soit à la fois cohérent et convenable, et dans lequel les grandeurs angle plan et angle solide soient considérées comme des grandeurs de base,

\section{considérant aussi}

- que l'interprétation donnée par le CIPM en 1969 pour la classe des unités supplémentaires introduite dans la Résolution 12 de la $11^{\mathrm{e}}$ Conférence générale des poids et mesures en 1960 laisse la liberté de traiter le radian et le stéradian comme unités de base dans le Système international,

- qu'une telle possibilité compromet la cohérence interne du Système international fondé sur sept unités de base seulement,

décide d'interpréter la classe des unités supplémentaires dans le Système international comme une classe d'unités dérivées sans dimension pour lesquelles la Conférence générale des poids et mesures laisse la liberté de les utiliser ou non dans les expressions des unités dérivées du Système international.
Le Comité international a estimé encore prématuré, en 1990, de choisir un symbole unique du litre.
* La classe des unités supplémentaires dans le SI a été abrogée en 1995 par décision de la $20^{\mathrm{e}} \mathrm{CGPM}$ (Résolution 8, voir p. 75). 
$17^{\mathrm{e}}$ CGPM, 1983

I Définition du mètre (CR, 97 et Metrologia, 1984, 20, 25)

\section{Résolution 1}

La Dix-septième Conférence générale des poids et mesures, considérant

- que la définition actuelle ne permet pas une réalisation du mètre suffisamment précise pour tous les besoins,

- que les progrès réalisés dans l'asservissement des lasers permettent d'obtenir des radiations plus reproductibles et plus faciles à utiliser que la radiation étalon émise par une lampe à krypton 86 ,

- que les progrès réalisés dans la mesure des fréquences et des longueurs d'onde de ces radiations ont abouti à des déterminations concordantes de la vitesse de la lumière dont l'exactitude est limitée principalement par la réalisation du mètre dans sa définition actuelle,

- que les valeurs des longueurs d'onde déterminées à partir de mesures de fréquence et d'une valeur donnée de la vitesse de la lumière ont une précision supérieure à celle qui peut être obtenue par comparaison avec la longueur d'onde de la radiation étalon du krypton 86,

- qu'il y a avantage, notamment pour l'astronomie et la géodésie, à maintenir inchangée la valeur de la vitesse de la lumière recommandée en 1975 par la $15^{\mathrm{e}}$ Conférence générale des poids et mesures, dans sa Résolution 2 ( $c=299792458 \mathrm{~m} / \mathrm{s}$ ),

- qu'une nouvelle définition du mètre a été envisagée sous diverses formes qui ont toutes pour effet de donner à la vitesse de la lumière une valeur exacte, égale à la valeur recommandée, et que cela n'introduit aucune discontinuité appréciable de l'unité de longueur, compte tenu de l'incertitude relative de $\pm 4 \times 10^{-9}$ des meilleures réalisations du mètre dans sa définition actuelle.

- que ces diverses formes, faisant appel soit au trajet parcouru par la lumière dans un intervalle de temps spécifié, soit à la longueur d'onde d'une radiation de fréquence mesurée ou de fréquence spécifiée, ont fait l'objet de consultations et de discussions approfondies, qu'elles ont été reconnues équivalentes et qu'un consensus s'est dégagé en faveur de la première forme,

- que le Comité consultatif pour la définition du mètre est dès maintenant en mesure de donner des instructions pour la mise en pratique d'une telle définition, instructions qui pourront inclure l'emploi de la radiation orangée du krypton 86 utilisée jusqu'ici comme étalon et qui pourront être complétées ou révisées par la suite,

\section{décide}

1. Le mètre est la longueur du trajet parcouru dans le vide par la lumière pendant une durée de 1/299 792458 de seconde.

2. La définition du mètre en vigueur depuis 1960 , fondée sur la transition entre les niveaux $2 p_{10}$ et $5 d_{5}$ de l'atome de krypton 86 , est abrogée.

I Sur la mise en pratique de la définition du mètre (CR, 98 et Metrologia, 1984, 20, 25-26)

\section{Résolution 2}

La Dix-septième Conférence générale des poids et mesures

invite le Comité international des poids et mesures

- à établir des instructions pour la mise en pratique de la nouvelle définition du mètre,

La valeur de l'incertitude donnée ici correspond à trois fois l'écart-type de la valeur en question.
Voir Recommandation 1 (CI-2002) du CIPM relative à la révision de la mise en pratique de la définition du mètre, p. 77. 
- à choisir des radiations qui puissent être recommandées comme étalons de longueur d'onde pour la mesure interférentielle des longueurs et à établir des instructions pour leur emploi,

- à poursuivre les études entreprises pour améliorer ces étalons.

\section{CIPM, 1984}

I Au sujet du sievert (PV, 52, 31 et Metrologia, 1985, 21, 90*

\section{Recommandation 1}

Le Comité international des poids et mesures,

considérant la confusion qui continue d'exister au sujet de la Résolution 5 , votée par la $16^{e}$ Conférence générale des poids et mesures (1979),

décide d'introduire l'explication suivante dans la Brochure « Le Système international d'unités (SI) » :

La grandeur équivalent de dose $H$ est le produit de la dose absorbée $D$ de rayonnements ionisants et de deux facteurs sans dimension $Q$ (facteur de qualité) et $N$ (produit de tous les autres facteurs de multiplication) prescrits par l'International Commission on Radiological Protection :

$$
H=Q \cdot N \cdot D \text {. }
$$

Ainsi, pour une radiation donnée, la valeur numérique de $H$ en joules par kilogramme peut être différente de la valeur numérique de $D$ en joules par kilogramme, puisqu'elle est fonction de la valeur de $Q$ et de $N$. Afin d'éviter tout risque de confusion entre la dose absorbée $D$ et l'équivalent de dose $H$, il faut employer les noms spéciaux pour les unités correspondantes, c'est-à-dire qu'il faut utiliser le nom gray au lieu de joule par kilogramme pour l'unité de dose absorbée $D$ et le nom sievert au lieu de joule par kilogramme pour l'unité d'équivalent de dose $H$.

\section{$18^{\mathrm{e}}$ CGPM, 1987}

I Ajustement prévu des représentations du volt et de l'ohm (CR, 100 et Metrologia, 1988, 25, 115)

\section{Résolution 6}

La Dix-huitième Conférence générale des poids et mesures,

\section{considérant}

- que l'uniformité mondiale et la constance à long terme des représentations nationales des unités électriques sont d'une importance majeure pour la science, le commerce et l'industrie du point de vue technique comme du point de vue économique,

- que de nombreux laboratoires nationaux utilisent l'effet Josephson et commencent à utiliser l'effet Hall quantique pour conserver respectivement des représentations du volt et de l'ohm qui donnent les meilleures garanties de stabilité à long terme,

- qu'en raison de l'importance de la cohérence entre les unités de mesure des diverses grandeurs physiques les valeurs attribuées à ces représentations doivent être autant que possible en accord avec le SI,

- que l'ensemble des résultats des expériences en cours ou récemment achevées permettra d'établir une valeur acceptable, suffisamment compatible avec le SI, pour le coefficient qui relie chacun de ces effets à l'unité électrique correspondante,
* Le CIPM a décidé en 2002 de modifier les explications sur la grandeur équivalent de dose dans la Brochure sur le SI (Recommandation 2 (CI-2002), voir p. 79). 
invite les laboratoires dont les travaux peuvent contribuer à établir la valeur du quotient de la tension par la fréquence dans l'effet Josephson et de la tension par le courant dans l'effet Hall quantique à poursuivre activement ces travaux et à communiquer sans délai leurs résultats au Comité international des poids et mesures et,

charge le Comité international des poids et mesures de recommander, dès qu'il le jugera possible, une valeur de chacun de ces quotients et une date à laquelle elle pourra être mise en pratique simultanément dans tous les pays ; cette valeur devrait être annoncée au moins un an à l'avance et pourrait être adoptée au $1^{\text {er }}$ janvier 1990.

\section{CIPM, 1988}

I Représentation du volt au moyen de l'effet Josephson (PV, 56, 19 et Metrologia, 1989, 26, 69)

\section{Recommandation 1}

Le Comité international des poids et mesures

agissant conformément aux instructions données dans la Résolution 6 de la $18^{\mathrm{e}}$ Conférence générale des poids et mesures concernant l'ajustement prévu des représentations du volt et de l'ohm,

\section{considérant}

- qu'une étude approfondie des résultats des déterminations les plus récentes conduit à une valeur de $483597,9 \mathrm{GHz} / \mathrm{V}$ pour la constante de Josephson, $K_{\mathrm{J}}$, c'est-à-dire pour le quotient de la fréquence par la tension correspondant au palier de rang $n=1$ dans l'effet Josephson,

- que l'effet Josephson, avec cette valeur de $K_{J}$, peut être utilisé pour établir un étalon de référence de force électromotrice dont l'incertitude (écart-type), par rapport au volt, est estimée à $4 \times 10^{-7}$ en valeur relative et dont la reproductibilité est nettement meilleure,

\section{recommande}

- que l'on adopte, par convention, pour la constante de Josephson, $K_{J}$, la valeur $K_{\mathrm{J}-90}=483597,9 \mathrm{GHz} / \mathrm{V}$ exactement,

- que cette nouvelle valeur soit utilisée à partir du $1^{\text {er }}$ janvier 1990 , et non auparavant, pour remplacer les valeurs actuellement en usage,

- que cette nouvelle valeur soit utilisée à partir de cette même date par tous les laboratoires qui fondent sur l'effet Josephson leurs mesures de force électromotrice,

- qu'à partir de cette même date tous les autres laboratoires ajustent la valeur de leurs étalons de référence pour la mettre en accord avec cette nouvelle valeur,

estime qu'aucun changement de cette valeur recommandée de la constante de Josephson ne sera nécessaire dans un avenir prévisible,

attire l'attention des laboratoires sur le fait que la nouvelle valeur est supérieure de $3,9 \mathrm{GHz} / \mathrm{N}$, soit approximativement $8 \times 10^{-6}$ en valeur relative, à la valeur donnée en 1972 par le Comité consultatif d'électricité dans sa Déclaration E-72. 
I Représentation de l'ohm au moyen de l'effet Hall quantique (PV, 56, 20 et Metrologia, 1989, 26, 70)

\section{Recommandation 2}

Le Comité international des poids et mesures,

agissant conformément aux instructions données dans la Résolution 6 de la $18^{\mathrm{e}}$ Conférence générale des poids et mesures concernant l'ajustement prévu des représentations du volt et de l'ohm,

\section{considérant}

- que la plupart des étalons actuels de référence de résistance électrique présentent au cours du temps des variations significatives,

- qu'un étalon de référence de résistance électrique fondé sur l'effet Hall quantique serait stable et reproductible,

- qu'une étude approfondie des résultats des déterminations les plus récentes conduit à une valeur de $25812,807 \Omega$ pour la constante de von Klitzing, $R_{\mathrm{k}}$, c'est-à-dire pour le quotient de la tension de Hall par le courant correspondant au plateau de rang $i=1$ dans l'effet Hall quantique,

- que l'effet Hall quantique, avec cette valeur de $R_{\mathrm{K}}$, peut être utilisé pour établir un étalon de référence de résistance dont l'incertitude (écart-type), par rapport à l'ohm, est estimée $2 \times 10^{-7}$ en valeur relative et dont la reproductibilité est nettement meilleure,

\section{recommande}

- que l'on adopte par convention, pour la constante de von Klitzing, $R_{\mathrm{K}}$, la valeur $R_{\mathrm{K}-90}=25812,807 \Omega$ exactement,

- que cette valeur soit utilisée à partir du $1^{\mathrm{er}}$ janvier 1990 , et non auparavant, par tous les laboratoires qui fondent sur l'effet Hall quantique leurs mesures de résistance électrique,

- qu'à partir de cette même date tous les autres laboratoires ajustent la valeur de leurs étalons de référence pour la mettre en accord avec $R_{\mathrm{K}-90}$,

- que, pour établir un étalon de référence de résistance électrique fondé sur l'effet Hall quantique, les laboratoires suivent les conseils pour la mise en oeuvre de la résistance de Hall quantifiée élaborés par le Comité consultatif d'électricité et publiés par les soins du Bureau international des poids et mesures, dans leur édition la plus récente,

et estime qu'aucun changement de cette valeur recommandée de la constante de von Klitzing ne sera nécessaire dans un avenir prévisible.

\section{CIPM, 1989}

I L’Échelle internationale de température de 1990 (PV, 57, 26 et Metrologia, 1990, 27, 13)

\section{Recommandation 5}

Le Comité international des poids et mesures (CIPM), conformément à l'invitation formulée par la $18^{\mathrm{e}}$ Conférence générale des poids et mesures en 1987 (Résolution 7), a adopté l'Échelle internationale de température de 1990 (EIT-90) en remplacement de l'Échelle internationale pratique de température de 1968 (EIPT-68).

Le CIPM souligne que, par rapport à l'EIPT-68, l'EIT-90

- s'étend vers des températures plus basses, jusqu'à $0,65 \mathrm{~K}$, et remplace, de ce fait, aussi l'Échelle provisoire de température de 1976 (EPT-76),

- est en bien meilleur accord avec les températures thermodynamiques correspondantes,
Lors de sa $89^{\mathrm{e}}$ session en 2000, le CIPM a approuvé la déclaration de la $22^{\mathrm{e}}$ session du CCEM concernant la valeur de la constante de von Klitzing, voir p. 77. 
- a une continuité, une précision et une reproductibilité nettement améliorées sur toute son étendue,

- comporte des sous-domaines et donne, dans certains domaines, des définitions équivalentes qui facilitent grandement son utilisation.

Le CIPM note de plus, que le texte de l'EIT-90 sera accompagné de deux documents, Supplementary Information for the ITS-90 et Techniques for Approximating the ITS-90, qui seront publiés par le Bureau international des poids et mesures et remis à jour périodiquement.

Le CIPM recommande

- que l'EIT-90 soit mise en application le $1^{\mathrm{er}}$ janvier 1990 ,

- et que, à la même date, l'EIPT-68 et l'EPT-76 soient abrogées.

$19^{\mathrm{e}}$ CGPM, 1991

I Préfixes SI zetta, zepto, yotta et yocto (CR, 97 et Metrologia, 1992, 29, 3)

\section{Résolution 4}

La $19^{\mathrm{e}}$ Conférence générale des poids et mesures (CGPM),

décide d'ajouter à la liste des préfixes SI pour la formation des noms des multiples et sous-multiples des unités, adoptée par la $11^{\mathrm{e}}$ CGPM, Résolution 12 , paragraphe 3 , la $12^{\mathrm{e}}$ CGPM, Résolution 8 et la $15^{\mathrm{e}}$ CGPM, Résolution 10 , les préfixes suivants :

Facteur par lequel

l'unité est multipliée

Préfixe Symbole

\begin{tabular}{lll}
\hline $10^{21}$ & zetta & $Z$ \\
$10^{-21}$ & zepto & $\mathrm{Z}$ \\
$10^{24}$ & yotta & $\mathrm{Y}$ \\
$10^{-24}$ & yocto & $\mathrm{y}$
\end{tabular}

Les noms zepto et zetta évoquent le chiffre sept (septième puissance de $10^{3}$ ) et la lettre $« \mathrm{z} »$ remplace la lettre « $\mathrm{s} »$ pour éviter le double emploi de la lettre « $\mathrm{s}$ » comme symbole. Les noms yocto et yotta sont dérivés de octo, qui évoque le chiffre huit (huitième puissance de $10^{3}$ ); la lettre « y » est ajoutée pour éviter l'emploi de la lettre « 0 » comme symbole à cause de la confusion possible avec le chiffre zéro.

\section{$20^{\mathrm{e}}$ CGPM, 1995}

I Suppression de la classe des unités supplémentaires dans le SI (CR, 121 et Metrologia, 1996, 33, 83)

\section{Résolution 8}

La $20^{\circ}$ Conférence générale des poids et mesures,

\section{considérant}

- que la $11^{\mathrm{e}}$ Conférence générale, en 1960, dans sa Résolution 12 établissant le Système international d'unités, SI, a distingué trois classes d'unités, celle des unités de base, celle des unités dérivées et celle des unités supplémentaires, cette dernière comprenant seulement le radian et le stéradian,

- que le statut des unités supplémentaires par rapport aux unités de base et aux unités dérivées, a donné lieu à des discussions,

- que le Comité international des poids et mesures (CIPM), en 1980, constatant que le statut ambigu des unités supplémentaires compromet la cohérence interne du SI, a interprété dans sa Recommandation 1 ( $\mathrm{Cl}-1980)$ les unités supplémentaires, dans le $\mathrm{SI}$, comme des unités dérivées sans dimension,

approuvant l'interprétation donnée par le CIPM en 1980, 


\section{décide}

- d'interpréter les unités supplémentaires, dans le SI, c'est-à-dire le radian et le stéradian, comme des unités dérivées sans dimension dont les noms et les symboles peuvent être utilisés, mais pas nécessairement, dans les expressions d'autres unités dérivées SI, suivant les besoins,

- et, par conséquent, de supprimer la classe des unités supplémentaires en tant que classe séparée dans le SI.

\section{$21^{\mathrm{e}}$ CGPM, 1999}

I La définition du kilogramme (CR, 141-142 et Metrologia, 2000, 37, 94)

\section{Résolution 7}

La $21^{\mathrm{e}}$ Conférence générale des poids et mesures,

\section{considérant}

- le besoin d'assurer la stabilité à long terme du Système international d'unités (SI),

- l'incertitude intrinsèque relative à la stabilité à long terme du prototype qui sert à définir l'unité de masse, l'une des unités de base du SI,

- que cette incertitude se répercute sur la stabilité à long terme des trois autres unités de base du SI, nommément l'ampère, la mole et la candela, dont la définition dépend de celle du kilogramme,

- les progrès déjà obtenus dans différentes expériences destinées à relier l'unité de masse à des constantes fondamentales ou atomiques,

- qu'il est souhaitable de disposer de plusieurs méthodes pour réaliser ce lien,

recommande que les laboratoires nationaux poursuivent leurs efforts pour affiner les expériences qui relient l'unité de masse à des constantes fondamentales ou atomiques et qui pourraient, dans l'avenir, servir de base à une nouvelle définition du kilogramme.

\section{Nom spécial donné à l'unité SI mole par seconde, le katal, pour exprimer} l'activité catalytique (CR, 145 et Metrologia, 2000, 37, 95)

\section{Résolution 12}

La $21^{\mathrm{e}}$ Conférence générale des poids et mesures,

\section{considérant}

- l'importance pour la santé humaine et la sécurité de faciliter l'emploi des unités du Système international d'unités (SI) dans les domaines de la médecine et de la biochimie

- qu'une unité en dehors du SI appelée « unité » représentée par le symbole U, qui est égale à $1 \mu \mathrm{mol} \cdot \mathrm{min}^{-1}$, et qui n'est pas cohérente avec le $\mathrm{SI}$, a été largement répandue en médecine et en biochimie depuis 1964 pour exprimer l'activité catalytique,

- que l'absence d'un nom spécial pour désigner l'unité dérivée et cohérente du SI qu'est la mole par seconde a conduit à ce que des résultats de mesures cliniques soient donnés en différentes unités locales,

- que l'emploi des unités SI en médecine et en chimie clinique est vivement recommandé par les unions internationales de ces domaines,

- que la Fédération internationale de chimie clinique et médecine de laboratoire a demandé au Comité consultatif des unités de recommander le nom spécial katal, symbole kat, pour l'unité SI mole par seconde,

- que tandis que la prolifération de noms spéciaux représente un danger pour le $\mathrm{SI}$, il existe des exceptions pour certains sujets liés à la santé humaine et à la sécurité 
( $15^{\mathrm{e}}$ Conférence générale, 1975 , Résolutions 8 et $9,16^{\mathrm{e}}$ Conférence générale, 1979, Résolution 5),

notant que le nom katal, symbole kat, est utilisé pour l'unité SI mole par seconde depuis plus de trente ans, pour exprimer l'activité catalytique,

décide d'adopter le nom spécial katal, symbole kat, pour l'unité SI mole par seconde pour exprimer l'activité catalytique, particulièrement dans les domaines de la médecine et de la biochimie,

et recommande que, lorsque le katal est utilisé, le mesurande soit spécifié en faisant référence au mode opératoire de mesure ; le mode opératoire de mesure doit mentionner le produit indicateur de la réaction mesurée.

CIPM, 2000

I " utilisation de la valeur de la constante de von Klitzing pour exprimer la valeur d'un étalon de référence de résistance en fonction de l'effet Hall quantique » $(P V, 68,34)$

Lors de sa $89^{\mathrm{e}}$ session en 2000, le CIPM a approuvé la déclaration suivante de la $22^{\mathrm{e}}$ session du CCEM (CCEM, 22, 22) :

"Le CCEM, considérant le récent ajustement des valeurs des constantes fondamentales recommandé par CODATA en 1998, estime que la valeur de la constante de von Klitzing, $R_{\mathrm{K}-90}$, peut être utilisée pour exprimer la valeur d'un étalon de référence de résistance en fonction de l'effet Hall quantique, avec une incertitude relative de $1 \times 10^{-7}$ par rapport à l'ohm (un écart-type), incertitude deux fois plus faible que celle qui avait été admise en 1988. »

\section{CIPM, 2001}

I « unités $\mathrm{SI}$ » et « unités du SI » (PV, 69, 38-39)

Le Comité international a approuvé en 2001 la proposition suivante du CCU concernant les « unités SI » et les « unités du SI » :

"Nous suggérons que les termes «unité $\mathrm{SI}$ » et "unité du SI » fassent tous deux référence aux unités de base et aux unités cohérentes dérivées, ainsi qu'à toutes les unités obtenues en les combinant aux préfixes recommandés des multiples et sous-multiples.

Nous suggérons que le terme " unité cohérente du SI » soit utilisé quand nous désirons restreindre son sens aux seules unités de base et aux unités cohérentes dérivées du SI. »

CIPM, 2002

I Révision de la mise en pratique de la définition du mètre (PV, 70, 90-93 et Metrologia, 40, 103-133)

\section{Recommandation 1}

Le Comité international des poids et mesures,

rappelant

- qu'en 1983 la $17^{\mathrm{e}}$ Conférence générale des poids et mesures (CGPM) a adopté une nouvelle définition du mètre,

- qu'à la même date la Conférence générale a invité le Comité international des poids et mesures (CIPM)

- à établir des instructions pour la réalisation pratique de la nouvelle définition du mètre (la mise en pratique), 
- à choisir des radiations qui puissent être recommandées comme étalons de longueur d'onde pour la mesure interférentielle des longueurs et à établir des instructions pour leur emploi,

- à poursuivre les études entreprises pour améliorer ces étalons et à compléter ou réviser par la suite ces instructions,

- qu'en réponse à cette invitation le CIPM a adopté la Recommandation 1 (Cl-1983) (mise en pratique de la définition du mètre) avec pour effet

- que le mètre soit réalisé par l'une des méthodes suivantes :

a) au moyen de la longueur $I$ du trajet parcouru dans le vide par une onde électromagnétique plane pendant la durée $t$; cette longueur est obtenue à partir de la mesure de la durée $t$, en utilisant la relation $I=c_{0} \cdot t$ et la valeur de la vitesse de la lumière dans le vide $c_{0}=299792458 \mathrm{~m} / \mathrm{s}$,

b) au moyen de la longueur d'onde dans le vide $\lambda$ d'une onde électromagnétique plane de fréquence $f$; cette longueur d'onde est obtenue à partir de la mesure de la fréquence $f$, en utilisant la relation $\lambda=c_{0} / f$ et la valeur de la vitesse de la lumière dans le vide $c_{0}=299792458 \mathrm{~m} / \mathrm{s}$,

c) au moyen de l'une des radiations de la liste ci-dessous, radiations pour lesquelles on peut utiliser la valeur donnée de la longueur d'onde dans le vide ou de la fréquence, avec l'incertitude indiquée, pourvu que l'on observe les conditions spécifiées et le mode opératoire reconnu comme approprié ;

- que dans tous les cas les corrections nécessaires soient appliquées pour tenir compte des conditions réelles telles que diffraction, gravitation ou imperfection du vide ;

- que dans le contexte de la relativité générale, le mètre est considéré comme une unité de longueur propre. Sa définition s'applique donc seulement dans un domaine spatial suffisamment petit, pour lequel les effets de la non-uniformité du champ gravitationnel peuvent être ignorés (notons, qu'à la surface de la Terre, cet effet est d'environ $1 \times 10^{-16}$ par mètre d'altitude en valeur relative). Dans ce cas, les seuls effets à prendre en compte sont ceux de la relativité restreinte. Les méthodes locales, préconisées en $b$ ) et $c$ ) pour réaliser le mètre, fournissent le mètre propre, mais la méthode préconisée en a) ne le permet pas nécessairement. La méthode préconisée en a) devrait donc être restreinte aux longueurs / suffisamment courtes pour que les effets prévus par la relativité générale soient négligeables par rapport aux incertitudes de mesure. Si ce n'est pas le cas, il convient de se référer au rapport du Groupe de travail du Comité consultatif du temps et des fréquences (CCTF) sur l'application de la relativité générale à la métrologie pour l'interprétation des mesures (Application of general relativity to metrology, Metrologia, 1997, 34, 261-290),

- que le CIPM avait recommandé une liste de radiations à cet effet ;

rappelant aussi qu'en 1992 et en 1997 le CIPM a révisé la mise en pratique de la définition du mètre ;

\section{considérant}

- que la science et les techniques continuent à exiger une meilleure exactitude dans la réalisation du mètre ;

- que, depuis 1997, les travaux effectués dans les laboratoires nationaux, au BIPM et dans d'autres laboratoires ont permis d'identifier de nouvelles radiations et des méthodes pour leur mise en œuvre qui conduisent à de plus faibles incertitudes ;

- que l'on s'oriente de plus en plus vers des fréquences optiques pour les activités liées au temps, et que l'on continue à élargir le domaine d'application des radiations recommandées dans la mise en pratique, non seulement à la métrologie dimensionnelle et à la réalisation du mètre, mais aussi à la spectroscopie de haute résolution, à la physique atomique et moléculaire, aux constantes fondamentales et aux télécommunications ; 
- que l'on dispose maintenant d'un certain nombre de nouvelles valeurs plus exactes de l'incertitude des fréquences de radiations d'atomes et d'ions refroidis très stables déjà mentionnées dans la liste de radiations recommandées, que la valeur de la fréquence de la radiation de plusieurs espèces d'atomes et d'ions refroidis a aussi été mesurée récemment, et que de nouvelles valeurs améliorées, et présentant des incertitudes réduites de manière significative, d'un certain nombre d'étalons de fréquence optique fondés sur des cuves à gaz ont été déterminées, y compris dans le domaine des longueurs d'ondes pour les télécommunications optiques ;

- que les nouvelles techniques de peigne à impulsions femtosecondes ont un intérêt manifeste pour relier la fréquence des étalons de fréquence optique très stables à celle des étalons de fréquence utilisés pour la réalisation de la seconde du Système international d'unités (SI), que ces techniques de mesure sont un moyen commode pour assurer la traçabilité au SI et peuvent fournir aussi bien des sources de fréquence que des techniques de mesure ;

reconnaît que les techniques de peigne arrivent au moment opportun et sont appropriées, et recommande de poursuivre les recherches pour étudier leurs possibilités ;

accueille favorablement les essais de validation en cours des techniques de peigne effectués par comparaison avec les autres techniques de chaînes de fréquence ;

encourage les laboratoires nationaux de métrologie et les autres laboratoires à poursuivre les études sur les techniques de peigne au plus haut niveau d'exactitude possible et à rechercher la simplicité pour encourager leur mise en pratique la plus étendue ;

\section{recommande}

- que la liste des radiations recommandées donnée par le CIPM en 1997 (Recommandation 1 (Cl-1997)) soit remplacée par la liste de radiations ci-dessous*, qui inclut ;

- des valeurs mises à jour de la fréquence des atomes de calcium et d'hydrogène refroidis et de l'ion piégé de strontium,

- la valeur de la fréquence de nouvelles espèces d'ions refroidis, y compris de l'ion piégé de $\mathrm{Hg}^{+}$, de l'ion piégé d' $\mathrm{In}^{+}$, et de l'ion piégé $\mathrm{d}^{\prime} \mathrm{Yb}^{+}$,

- des valeurs mises à jour de la fréquence de lasers asservis sur le rubidium, de lasers à grenat d'yttrium-aluminium dopé au néodyme ( $\mathrm{Nd}: \mathrm{YAG}$ ) et de lasers à hélium-néon ( $\mathrm{He}-\mathrm{Ne})$ asservis sur l'iode, de lasers à hélium-néon asservis sur le méthane, et de lasers à dioxyde de carbone asservis sur le tétroxyde d'osmium à $10 \mu \mathrm{m}$,

- des valeurs de la fréquence d'étalons pour les télécommunications optiques, y compris les lasers asservis sur le rubidium et l'acétylène.

$\cdots$

IÉquivalent de dose (PV, 70, 102)

\section{Recommandation 2}

Le Comité international des poids et mesures,

\section{considérant que}

- la définition actuelle de l'unité SI d'équivalent de dose (sievert) comprend un facteur « $N$ » (produit de tous les autres facteurs de multiplication) prescrit par l'International Commission on Radiological Protection (ICRP),

- I'ICRP et I'International Commission on Radiation Units and Measurements (ICRU) ont décidé de supprimer ce facteur $N$ qui n'est plus considéré comme nécessaire,

- la définition actuelle de l'équivalent de dose $H$ dans le Système international d'unités, qui comprend le facteur $N$, porte à confusion,

décide de modifier l'explication donnée dans la Brochure sur « Le Système International d'Unités (SI) » de la manière suivante :
* La liste des radiations recommandées, Recommandation 1 (CI-2002), figure dans les $\mathrm{PV}, \mathbf{7 0}, 93-101$ et dans Metrologia, 2003, 40, 104115. Des mises à jour sont disponibles sur le site Web du BIPM à l'adresse www.bipm.org/fr/ publications/mep.html.

Voir aussi J. Radiol. Prot., 2005, 25, 97-100. 
La grandeur équivalent de dose $H$ est le produit de la dose absorbée $D$ de rayonnements ionisants et du facteur sans dimension $Q$ (facteur de qualité) prescrit par l'ICRU, facteur défini en fonction du transfert d'énergie linéaire :

$$
H=Q \cdot D \text {. }
$$

Ainsi, pour une radiation donnée, la valeur numérique de $H$ en joules par kilogramme peut être différente de la valeur de $D$ en joules par kilogramme, puisqu'elle est fonction de la valeur de $Q$.

Le Comité décide donc de maintenir la dernière phrase de l'explication sous la forme suivante :

Afin d'éviter tout risque de confusion entre la dose absorbée $D$ et l'équivalent de dose $H$, il faut employer les noms spéciaux pour les unités correspondantes, c'est-à-dire qu'il faut utiliser le nom gray au lieu de joule par kilogramme pour l'unité de dose absorbée $D$ et le nom sievert au lieu de joule par kilogramme pour l'unité d'équivalent de dose $H$.

CIPM, 2003

I Révision de la liste des radiations recommandées pour la mise en pratique de la définition du mètre ( $P V, 71,70$ et Metrologia, 2004, 41, 99-100)

\section{Recommandation 1}

Le Comité international des poids et mesures,

\section{considérant que}

- l'on dispose depuis peu de meilleures valeurs des fréquences des radiations de certains étalons à ions refroidis très stables, déjà publiées dans la liste des radiations recommandées ;

- I'on a déterminé de meilleures valeurs des fréquences des étalons de fréquence optique dans l'infrarouge, fondés sur des cuves à gaz, dans le domaine des télécommunications optiques, valeurs déjà publiées dans la liste des radiations recommandées ;

- l'on a effectué récemment et pour la première fois des mesures de fréquence à l'aide de peignes à impulsions femtosecondes de certains étalons fondés sur des cuves à iode, qui figurent sur la liste complémentaire de radiations recommandées, mesures qui conduisent à une réduction considérable de l'incertitude ;

propose que la liste des radiations recommandées soit révisée pour inclure :

- les valeurs mises à jour des fréquences de la transition quadripolaire de l'ion piégé de ${ }^{88} \mathrm{Sr}^{+}$et de la transition octupolaire de l'ion piégé de ${ }^{171} \mathrm{Yb}^{+}$;

- la valeur mise à jour de la fréquence de l'étalon asservi sur l'acétylène à 1,54 $\mu \mathrm{m}$;

- des valeurs mises à jour de la fréquence d'étalons asservis sur l'iode à $543 \mathrm{~nm}$ et à $515 \mathrm{~nm}$.

$22^{\mathrm{e}}$ CGPM, 2003

ISymbole du séparateur décimal (CR, 169 et Metrologia, 2004, 41, 104)

\section{Résolution 10}

La $22^{\mathrm{e}}$ Conférence générale des poids et mesures,

considérant que

- l'un des principaux objectifs du Système international d'unités (SI) est de permettre d'exprimer la valeur des grandeurs d'une manière aisément compréhensible dans le monde entier,
Les mises à jour sont disponibles sur le site Web du BIPMà l'adresse www.bipm.org/fr/ publications/mep.html. 
- la valeur d'une grandeur est normalement exprimée par un nombre qui multiplie une unité,

- souvent le nombre utilisé pour exprimer la valeur d'une grandeur contient plusieurs chiffres, avec une partie entière et une partie décimale,

- la $9^{e}$ Conférence générale dans sa Résolution 7 (1948) avait décidé que «Dans les nombres, la virgule (usage français) ou le point (usage britannique) sont utilisés seulement pour séparer la partie entière des nombres de leur partie décimale »,

- conformément à la décision du Comité international des poids et mesures lors de sa $86^{\mathrm{e}}$ session (1997), le Bureau international des poids et mesures utilise maintenant le point (sur la ligne) comme séparateur décimal dans toutes les versions en anglais de ses publications, y compris dans le texte anglais de la Brochure sur le SI (la référence internationale sur le $\mathrm{SI}$ ), tout en continuant à utiliser la virgule (sur la ligne) comme séparateur décimal dans toutes ses publications en français,

- néanmoins certaines organisations internationales utilisent la virgule sur la ligne comme séparateur décimal dans leurs documents en anglais,

- de plus, certaines organisations internationales, y compris certaines organisations internationales de normalisation, spécifient que le séparateur décimal doit être la virgule sur la ligne, dans toutes les langues,

- la recommandation d'utiliser la virgule sur la ligne comme séparateur décimal est, dans de nombreuses langues, en conflit avec l'usage courant, qui consiste à utiliser le point sur la ligne,

- le fait d'utiliser le point sur la ligne ou la virgule sur la ligne comme séparateur décimal n'est pas toujours lié à la langue, car certains pays de même langue maternelle ont des usages différents, alors que d'autres pays pratiquant le plurilinguisme utilisent le point sur la ligne ou la virgule sur la ligne suivant la langue,

déclare que le symbole du séparateur décimal pourra être le point sur la ligne ou la virgule sur la ligne,

réaffirme que « Pour faciliter la lecture, les nombres peuvent être partagés en tranches de trois chiffres ; ces tranches ne sont jamais séparées par des points, ni par des virgules », comme le recommande la Résolution 7 de la $9^{\text {e }}$ Conférence générale de 1948.

\section{CIPM, 2005}

I Clarification de la définition du kelvin, unité de température thermodynamique (PV, 94, sous presse et Metrologia, 2006, 43, 177-178)

\section{Recommandation 2}

Le Comité international des poids et mesures (CIPM),

\section{considérant}

- que le kelvin, l'unité de température thermodynamique, est défini par la fraction $1 / 273,16$ de la température thermodynamique du point triple de l'eau,

- que la température du point triple de l'eau dépend des abondances relatives des isotopes de l'hydrogène et de l'oxygène présents dans l'échantillon d'eau utilisé,

- que cet effet est maintenant l'une des sources majeures d'écarts observés entre les différentes réalisations du point triple de l'eau,

\section{décide}

- que la définition du kelvin se réfère à une eau de composition isotopique spécifiée,

- que cette composition isotopique de l'eau soit la suivante :

0,000 15576 mole de ${ }^{2} \mathrm{H}$ par mole de ${ }^{1} \mathrm{H}$,

0,0003799 mole de ${ }^{17} \mathrm{O}$ par mole de ${ }^{16} \mathrm{O}$, et 
0,0020052 mole de ${ }^{18} \mathrm{O}$ par mole de ${ }^{16} \mathrm{O}$,

cette composition étant celle du matériau de référence de l'Agence internationale de l'énergie atomique "Vienna Standard Mean Ocean Water (VSMOW) », recommandée par I'Union internationale de chimie pure et appliquée dans « Atomic Weights of the Elements: Review 2000 »,

- que cette composition soit définie dans une note attachée à la définition du kelvin dans la Brochure sur le SI de la manière suivante :

«Cette définition se réfère à l'eau de composition isotopique définie par les rapports de quantité de matière suivants : 0,000 15576 mole de ${ }^{2} \mathrm{H}$ par mole de ${ }^{1} \mathrm{H}, 0,0003799$ mole de ${ }^{17} \mathrm{O}$ par mole de ${ }^{16} \mathrm{O}$ et 0,0020052 mole de ${ }^{18} \mathrm{O}$ par mole de ${ }^{16} \mathrm{O} »$.

I Révision de la liste des radiations recommandées pour la mise en pratique de la définition du mètre (PV, 94, sous presse et Metrologia, 2006, 43, 178)

\section{Recommandation 3}

Le Comité international des poids et mesures,

\section{considérant que}

- l'on dispose de meilleures valeurs des fréquences des radiations de certains étalons à ion ou à atomes refroidis très stables, déjà publiées dans la liste des radiations recommandées ;

- I'on a déterminé de meilleures valeurs des fréquences des étalons de fréquence optique, fondés sur des cuves à gaz, dans le domaine des télécommunications optiques, dans l'infrarouge, valeurs déjà publiées dans la liste des radiations recommandées ;

- I'on a déterminé de meilleures valeurs des fréquences de certains étalons fondés sur des cuves à iode, valeurs déjà publiées dans la liste complémentaire des sources recommandées ;

- l'on a effectué pour la première fois des mesures de la fréquence de nouveaux atomes refroidis, d'atomes dans la région de l'infrarouge proche et de molécules dans le domaine des télécommunications optiques, à l'aide de peignes à impulsions femtosecondes ;

décide que la liste des radiations recommandées soit révisée pour y inclure :

- les valeurs mises à jour des fréquences des transitions quadripolaires de l'ion piégé de ${ }^{88} \mathrm{Sr}^{+}$, de l'ion piégé de ${ }^{199} \mathrm{Hg}^{+}$et de l'ion piégé de ${ }^{171} \mathrm{Yb}^{+}$;

- la valeur mise à jour de la fréquence de la transition de l'atome de calcium ;

- la valeur mise à jour de la fréquence de l'étalon asservi sur l'acétylène à 1,54 $\mu \mathrm{m}$;

- la valeur mise à jour de la fréquence de l'étalon asservi sur l'iode à $515 \mathrm{~nm}$;

- la fréquence de la transition de l'atome de ${ }^{87} \mathrm{Sr}$ à $698 \mathrm{~nm}$;

- les fréquences des transitions de l'atome de ${ }^{87} \mathrm{Rb}$ autour de $760 \mathrm{~nm}$;

- les fréquences des transitions de la bande $(v 1+v 3)$ de ${ }^{12} \mathrm{C}_{2} \mathrm{H}_{2}$, et des bandes $(v 1+v 3)$ et $(v 1+v 3+v 4+v 5)$ de ${ }^{13} \mathrm{C}_{2} \mathrm{H}_{2}$, autour de $1,54 \mu \mathrm{m}$. 
Annexe 2. Réalisation pratique des définitions des principales unités

L'annexe 2 est publiée uniquement sous forme électronique sur le site Web du BIPM. Elle est disponible à l'adresse : www.bipm.org/fr/si/si brochure/appendix 2/. 



\section{Annexe 3. Unités pour la mesure des grandeurs photochimiques et photobiologiques}

Les rayonnements optiques sont susceptibles de produire des modifications chimiques dans certains matériaux vivants ou inertes. Cette propriété est appelée actinisme et les rayonnements capables de causer de tels changements sont connus sous le nom de rayonnements actiniques. Les rayonnements actiniques ont la propriété fondamentale qu'à l'échelle moléculaire un photon interagit avec une molécule pour altérer ou briser cette molécule en de nouvelles espèces moléculaires. Il est donc possible de définir des grandeurs photochimiques ou photobiologiques spécifiques en fonction de l'effet du rayonnement optique sur les récepteurs chimiques ou biologiques correspondants.

Dans le domaine de la métrologie, la seule grandeur photobiologique qui ait été formellement définie du point de vue des mesures selon les règles du SI est l'interaction de la lumière avec l'œil humain dans la vision. Une unité de base du SI, la candela, a été définie pour cette importante grandeur photobiologique. Plusieurs autres grandeurs photométriques, dont les unités sont dérivées de la candela, ont également été définies (comme le lumen ou le lux, voir tableau 3, chapitre 2, p. 28).

\section{Spectre d'action actinique}

Un rayonnement optique peut être caractérisé par sa distribution spectrale de puissance. Les mécanismes selon lesquels le rayonnement optique est absorbé par le système chimique ou biologique sont en général très complexes, et sont toujours fonction de la longueur d'onde (ou de la fréquence). Pour les applications métrologiques, cependant, la complexité des mécanismes d'absorption peut être ignorée et l'effet actinique est simplement caractérisé par un spectre d'action actinique qui relie la réponse photochimique ou photobiologique au rayonnement incident. Ce spectre d'action actinique décrit l'efficacité relative d'un rayonnement optique monochromatique à la longueur d'onde $\lambda$ à produire une réponse actinique donnée. Il est donné en valeur relative, normalisée à 1 pour le maximum de l'efficacité. Généralement, les spectres d'action actinique sont définis et recommandés par les organisations internationales scientifiques ou de normalisation.

Pour la vision, deux spectres d'action ont été définis par la CIE et approuvés par le CIPM : $V(\lambda)$ pour la vision photopique et $V^{\prime}(\lambda)$ pour la vision scotopique. Ces spectres d'action sont utilisés dans les mesures des grandeurs photométriques et font implicitement partie de la définition de l'unité SI pour la photométrie, la candela. La vision photopique est détectée sur la rétine de l'œil par les cônes, sensibles aux luminances élevées $\left(L>\right.$ environ $\left.10 \mathrm{~cd} \mathrm{~m}^{-2}\right)$, qui correspondent à la vision diurne. $\mathrm{La}$ vision scotopique est détectée sur la rétine de l'œil par les bâtonnets, sensibles aux faibles luminances ( $L<$ environ $10^{-3} \mathrm{~cd} \mathrm{~m}^{-2}$ ), qui correspondent à la vision nocturne. Dans le cas de luminances intermédiaires entre la vision photopique et la vision
Les définitions des grandeurs et des unités photométriques sont publiées dans le

Vocabulaire international de l'éclairage, publication CIE 17.4 (1987), ou dans le Vocabulaire électrotechnique international, publication CEI 50, chapitre 845 : Éclairage.
Principes régissant la photométrie, Monographie BIPM, 1983, 32 pp. 
scotopique, les cônes et les bâtonnets fonctionnent simultanément, on est alors dans le domaine de la vision mésopique.

Des spectres d'action ont également été définis par la CIE pour d'autres effets actiniques, tel que le spectre d'action de l'érythème (rougissement de la peau) dû au rayonnement ultraviolet. Ces spectres n'ont cependant pas de statut particulier dans le SI.

\section{Mesure des grandeurs photochimiques et photobiologiques ;} unités correspondantes

Les grandeurs et unités photométriques actuellement en usage dans le domaine de la vision sont bien établies et très largement utilisées depuis fort longtemps. Elles ne sont pas concernées par les règles énoncées dans la suite de ce texte. Pour toutes les autres grandeurs photochimiques ou photobiologiques, les règles suivantes doivent être appliquées pour la définition des unités à utiliser.

Une grandeur photochimique ou photobiologique est définie d'une manière purement physique comme une grandeur dérivée de la grandeur radiométrique correspondante, par l'évaluation de l'effet du rayonnement selon son action sur un récepteur sélectif. La sensibilité spectrale de ce récepteur est définie par le spectre d'action de l'effet photochimique ou photobiologique considéré. La grandeur est donnée par l'intégrale par rapport à la longueur d'onde de la distribution spectrale de la grandeur radiométrique considérée, pondérée par le spectre d'action actinique approprié. L'utilisation d'une intégrale suppose implicitement l'additivité arithmétique des grandeurs actiniques; dans la pratique, cette loi n'est pas parfaitement vérifiée. Le spectre d'action est donné en valeur relative ; c'est une grandeur sans dimension dont l'unité SI est le nombre un. La grandeur radiométrique correspondante a sa propre unité radiométrique. Ainsi, selon la règle d'obtention de l'unité SI d'une grandeur dérivée, l'unité de la grandeur photochimique ou photobiologique est celle de la grandeur radiométrique correspondante. Quand on donne un résultat numérique, il est indispensable de spécifier s'il s'agit d'une grandeur radiométrique ou d'une grandeur actinique puisque les unités sont les mêmes. Si pour un effet actinique il existe plusieurs spectres d'action, le spectre d'action utilisé pour les mesures doit être clairement spécifié.

Cette façon de définir les unités à utiliser pour les grandeurs photochimiques et photobiologiques a été recommandée par le Comité consultatif de photométrie et radiométrie lors de sa $9^{\mathrm{e}}$ session en 1977.

À titre d'exemple, l'éclairement érythémal effectif $E_{\text {er }}$ d'une source de rayonnement ultraviolet est obtenu en pondérant l'éclairement énergétique spectrique du rayonnement à la longueur $\lambda$ par l'efficacité de ce rayonnement à provoquer un érythème à cette longueur d'onde, et en sommant sur l'ensemble de toutes les longueurs d'onde présentes dans le spectre de la source. Ceci peut s'exprimer sous la forme mathématique suivante :

$$
E_{\text {er }}=\int E_{\lambda} s_{\text {er }}(\lambda) \mathrm{d} \lambda
$$

où $E_{\lambda}$ est l'éclairement énergétique spectrique en $\mathrm{W} \mathrm{m}^{-2} \mathrm{~nm}^{-1}$ à la longueur d'onde $\lambda$ et $s_{\text {er }}(\lambda)$ le spectre d'action de l'érythème, normalisé à 1 à son maximum. Le résultat de cette détermination donne l'éclairement érythémal, $E_{e r}$, exprimé en $\mathrm{W} \mathrm{m}^{-2}$ selon les règles du SI. 


\section{Liste des sigles}

utilisés dans le présent volume*

\section{Sigles des laboratoires, commissions et conférences}

\begin{tabular}{|c|c|}
\hline BAAS & British Association for the Advancement of Science \\
\hline $\mathrm{BIH}$ & Bureau international de l'heure \\
\hline BIPM & Bureau international des poids et mesures \\
\hline CARICOM & Communauté des Caraïbes/Carribean Community \\
\hline CCAUV & Comité consultatif de l'acoustique, des ultrasons et des vibrations \\
\hline CCDS* & Comité consultatif pour la définition de la seconde, voir CCTF \\
\hline $\mathrm{CCE}^{*}$ & Comité consultatif d'électricité, voir CCEM \\
\hline CCEM & $\begin{array}{l}\text { (auparavant dénommé CCE) Comité consultatif d'électricité et } \\
\text { magnétisme }\end{array}$ \\
\hline CCL & Comité consultatif des longueurs \\
\hline $\mathrm{CCM}$ & Comité consultatif pour la masse et les grandeurs apparentées \\
\hline CCPR & Comité consultatif de photométrie et radiométrie \\
\hline CCQM & Comité consultatif pour la quantité de matière : métrologie en chimie \\
\hline CCRI & Comité consultatif des rayonnements ionisants \\
\hline $\mathrm{CCT}$ & Comité consultatif de thermométrie \\
\hline $\mathrm{CCTF}$ & $\begin{array}{l}\text { (auparavant dénommé CCDS) Comité consultatif du temps et des } \\
\text { fréquences }\end{array}$ \\
\hline $\mathrm{CCU}$ & Comité consultatif des unités \\
\hline CEI & Commission électrotechnique internationale \\
\hline CGPM & Conférence générale des poids et mesures \\
\hline CIE & Commission internationale de l'éclairage \\
\hline CIPM & Comité international des poids et mesures \\
\hline CODATA & Committee on Data for Science and Technology \\
\hline $\mathrm{CR}$ & Comptes rendus de la Conférence générale des poids et mesures \\
\hline ICRP & International Commission on Radiological Protection \\
\hline ICRU & International Commission on Radiation Units and Measurements \\
\hline IERS & $\begin{array}{l}\text { Service international de la rotation terrestre et des systèmes de } \\
\text { référence/International Earth Rotation and Reference Systems Service }\end{array}$ \\
\hline ISO & Organisation internationale de normalisation \\
\hline OIML & Organisation internationale de métrologie légale \\
\hline OMS & Organisation mondiale de la santé \\
\hline PV & Procès-verbaux du Comité international des poids et mesures \\
\hline SUNAMCO & $\begin{array}{l}\text { Commission for Symbols, Units, Nomenclature, Atomic Masses and } \\
\text { Fundamental Constants, UIPPA }\end{array}$ \\
\hline UAI & Union astronomique internationale \\
\hline UICPA & Union internationale de chimie pure et appliquée \\
\hline UIPPA & Union internationale de physique pure et appliquée \\
\hline
\end{tabular}

\footnotetext{
* Les laboratoires ou organisations marqués d'un astérisque soit n'existent plus soit figurent sous un autre sigle.
} 


\section{$2 \quad$ Sigles des termes scientifiques}

CGS Système d'unités cohérent fondé sur les trois unités mécaniques centimètre, gramme et seconde

EIPT-68 Échelle internationale pratique de température de 1968

EIT-90 Échelle internationale de température de 1990

EPT-76 Échelle provisoire de température de 1976

ITS-90* International Temperature Scale of 1990, voir EIT-90

MKS Système d'unités fondé sur les trois unités mécaniques mètre, kilogramme et seconde

MKSA Système d'unités quadri-dimensionnel fondé sur le mètre, le kilogramme, la seconde et l'ampère

SI Système international d'unités

TAI Temps atomique international

TCG Temps-coordonnée géocentrique

TT Temps terrestre

UTC Temps universel coordonné

VSMOW Vienna Standard Mean Ocean Water 


\section{Index}

Le numéro en caractère gras indique la page où se trouve la définition de l'unité ou du terme.

\section{A}

accélération due à la pesanteur $\left(g_{\mathrm{n}}\right), 53$

actinisme, 17,85

activité d'un radionucléide, 28,62

ampère (A), 14, 19-20, 23, 26, 54, 56, 57, 59,

60

atmosphère normale, $38, \mathbf{5 7}$

atome gramme, 24

atome de césium, niveaux hyperfins, 23

\section{B}

bar, 38,56

barn, 38

becquerel $(\mathrm{Bq}), 28,29,30,62,68$

bel (B), 38-39, 45

bohr, rayon de Bohr, 36-37

bougie nouvelle, 53

British Association for the Advancement of Science (BAAS), 19

\section{C}

calorie, 56

calcul formel, 42-43

candela (cd), 14, 20, 26, 56, 57, 59, 64, 68-69, 85

capacité thermique, 29, 42

CEI, norme 60027, 14

CGS, 19, 39, 55

CGS de Gauss, 15, 39

CGS-UEM, 15, 39

CGS-UES, 15, 39

charge électrique élémentaire, 36, 37

chiffres groupés par tranches de 3 chiffres, 44 , 81

chimie clinique, 25,76

CODATA, 37, 77 constante,

d'Avogadro, 25, 36

de Josephson $\left(K_{\mathrm{J}}, K_{\mathrm{J}-90}\right), 72,73$

de Planck réduite, 36,37

de von Klitzing $\left(R_{\mathrm{K}}, R_{\mathrm{K}-90}\right), 21,74,77$

magnétique, perméabilité du vide, 14, 23

Convention du Mètre, 18-20

coulomb (C), 28, 54, 56, 60

Coulomb, loi de, 14

courant électrique, 14-15, 23, 26, 54, 57, 59

curie $(\mathrm{Ci}), 62$

\section{D}

dalton (Da), 36-37

décibel (dB), 38-39, 45

degré Celsius $\left({ }^{\circ} \mathrm{C}\right), 24,28-29,42,44,55,56$

dose absorbée, 18, 28-30, 68, 69, 72, 80

dose, équivalent de, voir sievert

dyne (dyn), 39, 56

\section{E}

eau, composition isotopique, 24,81

échelle

de température thermodynamique, 57

internationale de température de 1990

(EIT-90), 74-75

effet

Hall (y compris Hall quantique), 21, 72,

74, 77

Josephson, 72, 73

électronvolt (eV), 36, 37

énergie de Hartree, 36, 37

équations électromagnétiques à quatre

grandeurs, 14

erg, 39, 56 


\section{$\mathbf{F}$}

facteur de rationalisation, 15

farad (F), 28, 54, 60

\section{G}

gal (Gal), 39

Gauss, 19

gauss $(\mathrm{G}), 39$

Giorgi, 20

gon, 35

grade, 35

gramme, $17,19,33,56,62-63$

grandeurs, 13

biologiques, 17

de base, 13-15, 26

de comptage, 16, 31

dérivées, 13, 15, 27-29

de dimension un, 16, 27, 30-31, 45

électromagnétiques, 14, 39

logarithmiques, 38-39, 45

photobiologiques, $17,85-86$

photochimiques, $17,85-86$

photométriques, 53, 64, 85-86

règles d'écriture, $42-45$

sans dimension, 16, 27, 30-31, 45-46, 70

symboles (recommandés), 15, 42-44

valeur numérique, 42-44

gray (Gy), 28, 30, 68, 72

\section{H}

Hartree (énergie de), hartree, 36, 37

hectare (ha), 35

henry $(\mathrm{H}), 28, \mathbf{5 4}, 56,60$

hertz (Hz), 28, 56, 60

heure (h), 33, 35, 56

\section{I}

incertitude, 45

intensité lumineuse, 15, 26, 53, 57, 59, 64, 6869

ISO

ISO/TC $12,14,70$

norme ISO 31, 12, 14, 43

norme ISO/IEC 80000, 14

J

joule (J), 16, 28, 30, 42, 54-55, 60

jour (d), 33, 35

\section{K}

katal (kat), 28, 76-77

kelvin (K), 14, 21, 23-24, 26, 63-64, 81

kibioctet, 32

kilogramme, 14, 19-20, 22, 26, 33, 52, 53, 57, 59,76

multiples et sous-multiples, 17, 33, 62-63

prototype international, 19, 22, 52

$\mathbf{L}$

législation sur les unités, 18

litre (L ou 1), 35, 41, 52, 56, 60-61, 62, 69-70

longueur, 14-15, 19, 22, 26, 52, 53, 57, 59

lumen $(\mathrm{lm}), 28,56,60$; nouveau, 53

lux (lx), 28, 56, 60

\section{M}

masse, 14, 15, 19, 22, 26, 33, 53, 57, 59, 62, 76

masse et poids, 53

masse de l'électron, 36, 37

Maxwell, 19

maxwell (Mx), 39

mésopique, voir vision mésopique

mètre $(\mathrm{m}), 14,19, \mathbf{2 2}, 41,52,53,57,58,59$,

71

prototype international, 19, 22, 52, 53, 58, 59

microseconde d'arc ( $\mu$ as), 33, 35

mille marin, 35, 38-39

milliseconde d'arc (mas), 33, 35

millimètre de mercure, 38

minute ( $\min ), 35$

MKS, 20, 54

MKSA, 20

mole (mol), 14, 20-21, 24-25, 26, 67

molécule gramme, 24

multiples du kilogramme, 17, 33, 62-63

multiples et sous-multiples, préfixes, 16-17, $32-33,59,62,68,75$

$\mathbf{N}$

néper (Np), 38-39, 45

newton $(\mathrm{N}), 23,28, \mathbf{5 4}, 56,60$

note historique, $18-20$ 


\section{0}

œrsted (Oe), 39

ohm $(\Omega), 19,23,28,41,54,56,60,72-73$, 74,77

OIML, 18

OMS, 18

\section{$\mathbf{P}$}

pascal $(\mathrm{Pa}), 28,41,66$

phot (ph), 39

photopique, voir vision photopique

physique atomique, 36

pied, 40

poids (voir à masse)

poids atomique, 24-25

poids moléculaire, $24-25$

point triple de l'eau, 24, 54-55, 57, 64, 81

poise $(\mathrm{P}), 39,56$

pouce, 40

pourcent, 46

ppb, 46

ppm, 46

ppt, 46

préfixes SI, 16-17, 19, 32-33, 35, 38-39, 41-

$42,59,62,65,68,75$

$\mathbf{Q}$

quantité de matière, 15, 24-25, 26, 67

quantité de chaleur, voir joule

\section{$\mathbf{R}$}

radian (rad), 28-29, 31, 45, 60, 70, 75-76

radiothérapie, 18

rayonnement actinique, $17,85-86$

rayonnements ionisants, 18, 30, 68, 69, 72, $79-80$

relativité générale, 17,78

\section{$\mathbf{S}$}

scotopique, voir vision scotopique seconde (s), 14, 19, 21-23, 26, 41, 56, 57-58,

59,63

seconde d'arc, 35

séparateur décimal, 12, 44-45, 80-81

SI, voir Système international d'unités

siemens (S), 28, 66

sievert (Sv), 28-29, 30, 69, 72, 79

stéradian (sr), 28-29, 31, 45, 60, 70, 75-76 stilb (sb), 39, 56

stokes (St), 39

symboles

dimensionnels, 15

écriture et emploi des, 41-46

des grandeurs, 15, 26, 42, 44-45

unités, 26, 41, 59

unités (obligatoires), 15, 26, 41-42

unités dérivées ayant des noms spéciaux, $16,27-30$

système

international d'unités (SI), 14, 55, 57,

58, 59 ; création, 55, 57, 58, 59

international de grandeurs, 14

métrique décimal, 19

$\mathbf{T}$

TAI, voir Temps atomique international température

Celsius, 24, 28, 44

thermodynamique, 14-15, 23-24, 26, 57, $59,63-64,81-82$

temps

durée, 13, 15, 22-23, 26, 57-58, 63

atomique international (TAI), 66-67

universel coordonné (UTC), 67-68

tesla $(\mathrm{T}), 28,60$

Thomson, 19

tonne, 35,56 ; métrique, 35

\section{U}

UICPA, 25 ; livre vert, 43

UIPPA SUNAMCO, 25 ; livre rouge ; 43

unité(s)

absolues, 23

acoustiques, 17

astronomique, 36-37

atomiques, 36-37

ayant des noms spéciaux et des symboles particuliers, 16, 27-30

CGS, 19, 39, 55

CGS de Gauss, 15, 39

CGS-UEM, 15, 39

CGS-UES, 15, 39

de base, 13, 21-26, 57, 59, 67 ;

définitions, 21-26

dérivées, 13, 27-30, 64-65

dérivées cohérentes, 16, 27-30, 77

électriques, 54 
en dehors du SI, 34-40

des grandeurs biologiques, 17-18

internationales OMS, 18

multiples et sous-multiples des, 17, 32-33,

$41,59,62,68,75$

naturelles, 36-37

noms, 42

photométriques, 53, 64, 85-86

pratiques, 19, 55-56, 57, 58

réalisation, $11, \mathbf{2 1}, 83$

règles d'écriture, 56

SI, 17, 21-30

supplémentaires, $60, \mathbf{6 5}, 70,75-76$

symboles, 26, 41-42, 56

UTC, voir Temps universel coordonné

V

viscosité

cinématique (stokes), 39

dynamique (poise), 39, 56

vision

mésopique, 69,86

photopique, 69,85

scotopique, 69, 85-86

vitesse de la lumière dans le vide, 22, 37, 78

volt (V), 28, 54, 56, 60, 72, 73

von Klitzing, voir constante de

\section{W}

watt (W), 28, 54, 56, 60

Weber, 19

weber (Wb), 28, 54, 60

$\mathbf{Y}$

yard, 40 


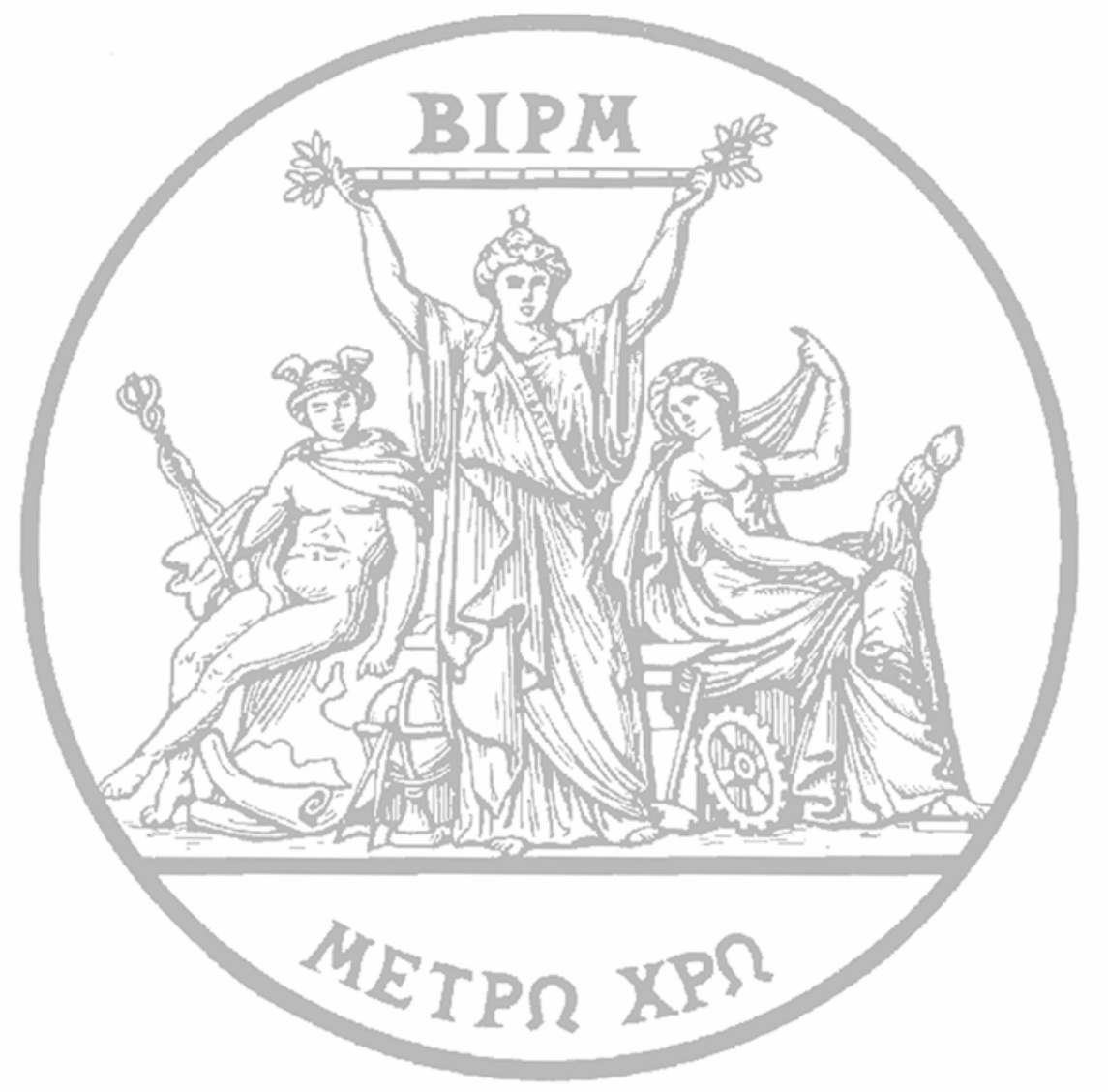



Bureau International des Poids et Mesures

\section{The International System of Units \\ (SI)}




\section{Note on the use of the English text}

To make its work more widely accessible, the International Committee for Weights and Measures has decided to publish an English version of its reports. Readers should note that the official record is always that of the French text. This must be used when an authoritative reference is required or when there is doubt about the interpretation of the text.

Translations, complete or partial, of this brochure (or of its earlier editions) have been published in various languages, notably in Bulgarian, Chinese, Czech, English, German, Japanese, Korean, Portuguese, Romanian, and Spanish. The ISO and numerous countries have also published standards and guides to the use of SI units. 


\section{The BIPM and the Metre Convention}

The International Bureau of Weights and Measures (BIPM) was set up by the Metre Convention signed in Paris on 20 May 1875 by seventeen States during the final session of the diplomatic Conference of the Metre. This Convention was amended in 1921.

The BIPM has its headquarters near Paris, in the grounds $\left(43520 \mathrm{~m}^{2}\right)$ of the Pavillon de Breteuil (Parc de Saint-Cloud) placed at its disposal by the French Government; its upkeep is financed jointly by the Member States of the Metre Convention.

The task of the BIPM is to ensure worldwide unification of measurements; its function is thus to:

- establish fundamental standards and scales for the measurement of the principal physical quantities and maintain the international prototypes;

- carry out comparisons of national and international standards;

- ensure the coordination of corresponding measurement techniques;

- carry out and coordinate measurements of the fundamental physical constants relevant to these activities.

The BIPM operates under the exclusive supervision of the International Committee for Weights and Measures (CIPM) which itself comes under the authority of the General Conference on Weights and Measures (CGPM) and reports to it on the work accomplished by the BIPM.

Delegates from all Member States of the Metre Convention attend the General Conference which, at present, meets every four years. The function of these meetings is to:

- discuss and initiate the arrangements required to ensure the propagation and improvement of the International System of Units (SI), which is the modern form of the metric system;

- confirm the results of new fundamental metrological determinations and various scientific resolutions of international scope;

- take all major decisions concerning the finance, organization and development of the BIPM.

The CIPM has eighteen members each from a different State: at present, it meets every year. The officers of this committee present an annual report on the administrative and financial position of the BIPM to the Governments of the Member States of the Metre Convention. The principal task of the CIPM is to ensure worldwide uniformity in units of measurement. It does this by direct action or by submitting proposals to the CGPM.
As of 31 December 2005, fifty-one States were members of this Convention: Argentina, Australia, Austria, Belgium, Brazil, Bulgaria, Cameroon, Canada, Chile, China, Czech Republic, Denmark, Dominican Republic, Egypt, Finland, France, Germany, Greece, Hungary, India, Indonesia, Iran (Islamic Rep. of), Ireland, Israel, Italy, Japan, Korea (Dem. People's Rep. of), Korea (Rep. of), Malaysia, Mexico, The Netherlands, New Zealand, Norway, Pakistan, Poland, Portugal, Romania, Russian

Federation, Serbia and Montenegro, Singapore, Slovakia, South Africa, Spain, Sweden, Switzerland, Thailand, Turkey, United Kingdom, United States, Uruguay, and Venezuela.

Twenty States and Economies were Associates of the General Conference: Belarus, CARICOM, Chinese Taipei, Costa Rica, Croatia, Cuba, Ecuador, Estonia, Hong Kong (China), Jamaica, Kazakhstan, Kenya, Latvia, Lithuania, Malta, Panama, Philippines, Slovenia, Ukraine, and Viet Nam. 
The activities of the BIPM, which in the beginning were limited to measurements of length and mass, and to metrological studies in relation to these quantities, have been extended to standards of measurement of electricity (1927), photometry and radiometry (1937), ionizing radiation (1960), time scales (1988) and to chemistry (2000). To this end the original laboratories, built in 1876-1878, were enlarged in 1929; new buildings were constructed in 1963-1964 for the ionizing radiation laboratories, in 1984 for the laser work and in 1988 for a library and offices. In 2001 a new building for the workshop, offices and meeting rooms was opened.

Some forty-five physicists and technicians work in the BIPM laboratories. They mainly conduct metrological research, international comparisons of realizations of units and calibrations of standards. An annual report, the Director's Report on the Activity and Management of the International Bureau of Weights and Measures, gives details of the work in progress.

Following the extension of the work entrusted to the BIPM in 1927, the CIPM has set up bodies, known as Consultative Committees, whose function is to provide it with information on matters that it refers to them for study and advice. These Consultative Committees, which may form temporary or permanent working groups to study special topics, are responsible for coordinating the international work carried out in their respective fields and for proposing recommendations to the CIPM concerning units.

The Consultative Committees have common regulations (BIPM Proc.-Verb. Com. Int. Poids et Mesures, 1963, 31, 97). They meet at irregular intervals. The president of each Consultative Committee is designated by the CIPM and is normally a member of the CIPM. The members of the Consultative Committees are metrology laboratories and specialized institutes, agreed by the CIPM, which send delegates of their choice. In addition, there are individual members appointed by the CIPM, and a representative of the BIPM (Criteria for membership of Consultative Committees, BIPM Proc.-Verb. Com. Int. Poids et Mesures, 1996, 64, 124). At present, there are ten such committees:

1. The Consultative Committee for Electricity and Magnetism (CCEM), new name given in 1997 to the Consultative Committee for Electricity (CCE) set up in 1927;

2. The Consultative Committee for Photometry and Radiometry (CCPR), new name given in 1971 to the Consultative Committee for Photometry (CCP) set up in 1933 (between 1930 and 1933 the CCE dealt with matters concerning photometry);

3. The Consultative Committee for Thermometry (CCT), set up in 1937;

4. The Consultative Committee for Length (CCL), new name given in 1997 to the Consultative Committee for the Definition of the Metre (CCDM), set up in 1952;

5. The Consultative Committee for Time and Frequency (CCTF), new name given in 1997 to the Consultative Committee for the Definition of the Second (CCDS) set up in 1956;

6. The Consultative Committee for Ionizing Radiation (CCRI), new name given in 1997 to the Consultative Committee for Standards of Ionizing Radiation (CCEMRI) set up in 1958 (in 1969 this committee established four sections: 
Section I (X- and $\gamma$-rays, electrons), Section II (Measurement of radionuclides), Section III (Neutron measurements), Section IV ( $\alpha$-energy standards); in 1975 this last section was dissolved and Section II was made responsible for its field of activity);

7. The Consultative Committee for Units (CCU), set up in 1964 (this committee replaced the Commission for the System of Units set up by the CIPM in 1954);

8. The Consultative Committee for Mass and Related Quantities (CCM), set up in 1980 ;

9. The Consultative Committee for Amount of Substance: Metrology in chemistry (CCQM), set up in 1993;

10. The Consultative Committee for Acoustics, Ultrasound and Vibration (CCAUV), set up un 1999.

The proceedings of the General Conference and the CIPM are published by the BIPM in the following series:

- Report of the meeting of the General Conference on Weights and Measures;

- Report of the meeting of the International Committee for Weights and Measures.

The CIPM decided in 2003 that the reports of meetings of the Consultative Committees should no longer be printed, but would be placed on the BIPM website, in their original language.

The BIPM also publishes monographs on special metrological subjects and, under the title The International System of Units (SI), a brochure, periodically updated, in which are collected all the decisions and recommendations concerning units.

The collection of the Travaux et Mémoires du Bureau International des Poids et Mesures (22 volumes published between 1881 and 1966) and the Recueil de Travaux du Bureau International des Poids et Mesures (11 volumes published between 1966 and 1988) ceased by a decision of the CIPM.

The scientific work of the BIPM is published in the open scientific literature and an annual list of publications appears in the Director's Report on the Activity and Management of the International Bureau of Weights and Measures.

Since 1965 Metrologia, an international journal published under the auspices of the CIPM, has printed articles dealing with scientific metrology, improvements in methods of measurement, work on standards and units, as well as reports concerning the activities, decisions and recommendations of the various bodies created under the Metre Convention. 
The BIPM and the Metre Convention $\quad 95$

$\begin{array}{ll}\text { Preface to the 8th edition } & 101\end{array}$

1 Introduction 103

$\begin{array}{lll}1.1 & \text { Quantities and units } & 103\end{array}$

1.2 The International System of Units (SI) and the corresponding

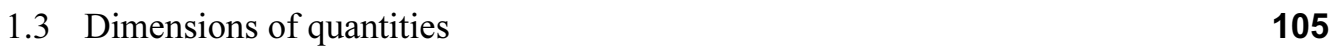

1.4 Coherent units, derived units with special names, and the SI prefixes 106

$\begin{array}{lll}1.5 & \text { SI units in the framework of general relativity } & 107\end{array}$

$\begin{array}{ll}1.6 & \text { Units for quantities that describe biological effects } \\ 1.7\end{array}$

$\begin{array}{ll}1.7 \text { Legislation on units } & 108\end{array}$

$\begin{array}{ll}1.8 \text { Historical note } & 108\end{array}$

2 SI units $\quad 111$

2.1 SI base units $\quad 111$

2.1.1 Definitions $\quad 111$

2.1.1.1 Unit of length (metre) 112

2.1.1.2 Unit of mass (kilogram) 112

2.1.1.3 Unit of time (second) $\quad 112$

2.1.1.4 Unit of electric current (ampere) 113

2.1.1.5 Unit of thermodynamic temperature (kelvin) 113

2.1.1.6 Unit of amount of substance (mole) $\quad 114$

2.1.1.7 Unit of luminous intensity (candela) $\quad 115$

2.1.2 Symbols for the seven base units $\quad 116$

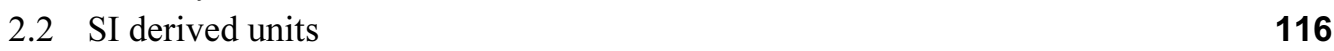

2.2.1 Derived units expressed in terms of base units $\quad 116$

2.2.2 Units with special names and symbols; units that incorporate
special names and symbols

2.2.3 Units for dimensionless quantities, also called

3 Decimal multiples and submultiples of SI units $\quad 121$

$\begin{array}{lll}3.1 & \text { SI prefixes } & 121\end{array}$

3.2 The kilogram 122 
4.1 Non-SI units accepted for use with the SI, and units based on fundamental constants

4.2 Other non-SI units not recommended for use

5 Writing unit symbols and names, and expressing the values of quantities

$\begin{array}{llr}5.1 & \text { Unit symbols } & 130\end{array}$

5.2 Unit names $\quad 131$

5.3 Rules and style conventions for expressing values of quantities $\quad 131$

5.3.1 Value and numerical value of a quantity, and the use of $\begin{array}{ll}\text { quantity calculus } & 131\end{array}$

5.3.2 Quantity symbols and unit symbols 132

5.3.3 Formatting the value of a quantity 133

5.3.4 Formatting numbers, and the decimal marker 133

5.3.5 Expressing the measurement uncertainty in the value of a quantity 133

5.3.6 Multiplying or dividing quantity symbols, the values of quantities, or numbers

5.3.7 Stating values of dimensionless quantities, or quantities of dimension one

Appendix 1. - Decisions of the CGPM and the CIPM

Appendix 2. - Practical realization of the definitions of some important units

Appendix 3. - Units for photochemical and photobiological quantities 173

$\begin{array}{ll}\text { List of acronyms } & 175\end{array}$

$\begin{array}{lr}\text { Index } & 177\end{array}$ 



\section{Preface to the 8th edition}

We have pleasure in introducing the 8th edition of this publication, commonly called the SI Brochure, which defines and presents the Système International d'Unités, the SI (known in English as the International System of Units). This Brochure is published as a hard copy, and is also available in electronic form at www.bipm.org/en/si/si brochure/.

Since 1970, the Bureau International des Poids et Mesures, the BIPM (known in English as the International Bureau of Weights and Measures), has published seven previous editions of this document. Its main purpose is to define and promote the SI, which has been used around the world as the preferred language of science and technology since its adoption in 1948 through a Resolution of the 9th Conférence Générale des Poids et Mesures, the CGPM (known in English as the General Conference on Weights and Measures).

The SI is, of course, a living system which evolves, and which reflects current best measurement practice. This 8 th edition therefore contains a number of changes since the previous edition. As before, it lists the definitions of all the base units, and all the Resolutions and Recommendations of the Conférence Générale des Poids et Mesures and the Comité International des Poids et Mesures, the CIPM (known in English as the International Committee for Weights and Measures), relating to the International System of Units. Formal reference to CGPM and CIPM decisions are to be found in the successive volumes of the Comptes Rendus of the CGPM (CR) and the ProcèsVerbaux of the CIPM (PV); many of these are also listed in Metrologia. To simplify practical use of the system, the text provides explanations of these decisions, and the first chapter provides a general introduction to establishing a system of units and to the SI in particular. The definitions and the practical realizations of all the units are also considered in the context of general relativity. A brief discussion of units associated with biological quantities has been introduced for the first time.

Appendix 1 reproduces, in chronological order, all the decisions (Resolutions, Recommendations, Declarations) promulgated since 1889 by the CGPM and the CIPM on units of measurement and the International System of Units.

Appendix 2 exists only in the electronic version, which is available at www.bipm.org/en/si/si brochure/appendix $2 /$. It outlines the practical realization of some important units, consistent with the definitions given in the principal text, which metrological laboratories can make to realize physical units and to calibrate material standards and measuring instruments of the highest quality. This appendix will be updated regularly to reflect improvements in the experimental techniques for realizing the units.

Appendix 3 presents units used to measure actinic effects in biological materials. 
The Comité Consultatif des Unités of the CIPM, the CCU (known in English as the Consultative Committee for Units), was responsible for drafting this document, and both the CCU and the CIPM approved the final text. This 8th edition is a revision of the 7th edition (1998); it takes into consideration decisions made by the CGPM and the CIPM since the 7 th edition was published.

For more than thirty-five years this document has been used as a work of reference in many countries, organizations, and scientific unions. To make its contents accessible to a greater number of readers, the CIPM decided, in 1985, to include an English version of the text in the 5th edition; this double presentation is continued in all later editions. For the first English version the BIPM endeavoured to produce a faithful translation of the French original by close collaboration with the National Physical Laboratory (Teddington, United Kingdom) and the National Institute of Standards and Technology (Gaithersburg, United States), at that time the National Bureau of Standards. For the present edition the French and English versions were prepared by the CCU in close collaboration with the BIPM.

The 22nd CGPM decided, in 2003, following a decision of the CIPM in 1997, that "the symbol for the decimal marker shall be either the point on the line or the comma on the line". Following this decision, and following custom in the two languages, in this edition the point on the line is used as a decimal marker in the English text, and a comma on the line is used in the French text. This has no implication for the translation of the decimal marker into other languages. A point to note is that small spelling variations occur in the language of the English speaking countries (for instance, "metre" and "meter", "litre" and "liter"). In this respect, the English text presented here follows the International Standard ISO 31, Quantities and Units.

Readers should note that the official record is always that of the French text. This must be used when an authoritative reference is required or when there is doubt about the interpretation of the text.

March 2006

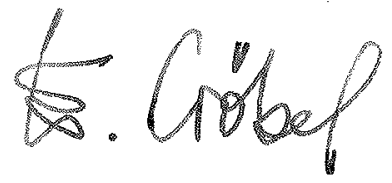

E. Göbel

President, CIPM

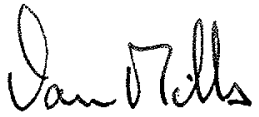

I. M. Mills

President, $\mathrm{CCU}$

\section{Anvew Wallowi}

A. J. Wallard

Director, BIPM 


\section{Introduction}

\subsection{Quantities and units}

The value of a quantity is generally expressed as the product of a number and a unit. The unit is simply a particular example of the quantity concerned which is used as a reference, and the number is the ratio of the value of the quantity to the unit. For a particular quantity, many different units may be used. For example, the speed $v$ of a particle may be expressed in the form $v=25 \mathrm{~m} / \mathrm{s}=90 \mathrm{~km} / \mathrm{h}$, where metre per second and kilometre per hour are alternative units for expressing the same value of the quantity speed. However, because of the importance of a set of well defined and easily accessible units universally agreed for the multitude of measurements that support today's complex society, units should be chosen so that they are readily available to all, are constant throughout time and space, and are easy to realize with high accuracy.

In order to establish a system of units, such as the International System of Units, the SI, it is necessary first to establish a system of quantities, including a set of equations defining the relations between those quantities. This is necessary because the equations between the quantities determine the equations relating the units, as described below. It is also convenient to choose definitions for a small number of units that we call base units, and then to define units for all other quantities as products of powers of the base units that we call derived units. In a similar way the corresponding quantities are described as base quantities and derived quantities, and the equations giving the derived quantities in terms of the base quantities are used to determine the expression for the derived units in terms of the base units, as discussed further in Section 1.4 below. Thus in a logical development of this subject, the choice of quantities and the equations relating the quantities comes first, and the choice of units comes second.

From a scientific point of view, the division of quantities into base quantities and derived quantities is a matter of convention, and is not essential to the physics of the subject. However for the corresponding units, it is important that the definition of each base unit is made with particular care, to satisfy the requirements outlined in the first paragraph above, since they provide the foundation for the entire system of units. The definitions of the derived units in terms of the base units then follow from the equations defining the derived quantities in terms of the base quantities. Thus the establishment of a system of units, which is the subject of this brochure, is intimately connected with the algebraic equations relating the corresponding quantities.

The number of derived quantities of interest in science and technology can, of course, be extended without limit. As new fields of science develop, new quantities are devised by researchers to represent the interests of the field, and with these new quantities come new equations relating them to those quantities that were previously familiar, and hence ultimately to the base quantities. In this way the derived units to
The terms quantity and unit are defined in the International Vocabulary of Basic and General Terms in Metrology, the VIM.

The quantity speed, $v$, may be expressed in terms of the quantities distance, $x$, and time, $t$, by the equation $v=\mathrm{d} x / \mathrm{d} t$.

In most systems of quantities and units, distance $x$ and time $t$ are regarded as base quantities, for which the metre, $\mathrm{m}$, and the second, s, may be chosen as base units. Speed $v$ is then taken as a derived quantity, with the derived unit metre per second, $\mathrm{m} / \mathrm{s}$.

For example, in electrochemistry, the electric mobility of an ion, $u$, is defined as the ratio of its velocity $v$ to the electric field strength, $E: u=v / E$. The derived unit of electric mobility is then given as $(\mathrm{m} / \mathrm{s}) /(\mathrm{V} / \mathrm{m})=\mathrm{m}^{2} \mathrm{~V}^{-1} \mathrm{~s}^{-1}$ in units which may be easily related to the chosen base units ( $\mathrm{V}$ is the symbol for the SI derived unit volt). 
be used with the new quantities may always be defined as products of powers of the previously chosen base units.

\subsection{The International System of Units (SI) and the corresponding system of quantities}

This Brochure is concerned with presenting the information necessary to define and use the International System of Units, universally known as the SI (from the French Système International d'Unités). The SI was established by and is defined by the General Conference on Weights and Measures, the CGPM, as described in the Historical note in Section 1.8 below*.

The system of quantities, including the equations relating the quantities, to be used with the SI, is in fact just the quantities and equations of physics that are familiar to all scientists, technologists, and engineers. They are listed in many textbooks and in many references, but any such list can only be a selection of the possible quantities and equations, which is without limit. Many of the quantities, their recommended names and symbols, and the equations relating them, are listed in the International Standards ISO 31 and IEC 60027 produced by Technical Committee 12 of the International Organization for Standardization, ISO/TC 12, and by Technical Committee 25 of the International Electrotechnical Commission, IEC/TC 25. The ISO 31 and IEC 60027 Standards are at present being revised by the two standardization organizations in collaboration. The revised harmonized standard will be known as ISO/IEC 80000, Quantities and Units, in which it is proposed that the quantities and equations used with the SI will be known as the International System of Quantities.

The base quantities used in the SI are length, mass, time, electric current, thermodynamic temperature, amount of substance, and luminous intensity. The base quantities are by convention assumed to be independent. The corresponding base units of the SI were chosen by the CGPM to be the metre, the kilogram, the second, the ampere, the kelvin, the mole, and the candela. The definitions of these base units are presented in Section 2.1.1 in the following chapter. The derived units of the SI are then formed as products of powers of the base units, according to the algebraic relations that define the corresponding derived quantities in terms of the base quantities, see Section 1.4 below.

On rare occasions a choice may arise between different forms of the relations between the quantities. An important example occurs in defining the electromagnetic quantities. In this case the rationalized four-quantity electromagnetic equations used with the SI are based on length, mass, time, and electric current. In these equations, the electric constant $\varepsilon_{0}$ (the permittivity of vacuum) and the magnetic constant $\mu_{0}$ (the permeability of vacuum) have dimensions and values such that $\varepsilon_{0} \mu_{0}=1 / c_{0}^{2}$, where $c_{0}$ is the speed of light in vacuum. The Coulomb law of electrostatic force between two particles with charges $q_{1}$ and $q_{2}$ separated by a distance $r$ is written**

$$
\boldsymbol{F}=\frac{q_{1} q_{2} \boldsymbol{r}}{4 \pi \varepsilon_{0} r^{3}}
$$

\footnotetext{
* Acronyms used in this Brochure are listed with their meaning on p. 175.

** Symbols in bold print are used to denote vectors.
}

The name Système International d'Unités, and the abbreviation SI, were established by the 11th CGPM in 1960 .

Examples of the equations relating quantities used in the SI are the Newtonian inertial equation relating force, $F$, to mass, $m$, and acceleration, $a$, for a particle: $F=m a$, and the equation giving the kinetic energy, $T$, of a particle moving with velocity, $v$ : $T=m v^{2} / 2$. 
and the corresponding equation for the magnetic force between two thin wire elements carrying electric currents, $i_{1} \mathrm{~d} l_{1}$ and $i_{2} \mathrm{~d} l_{2}$, is written

$$
\mathrm{d}^{2} \boldsymbol{F}=\frac{\mu_{0}}{4 \pi} \frac{i_{1} \mathrm{~d} \boldsymbol{l}_{1} \times\left(i_{2} \mathrm{~d} \boldsymbol{l}_{2} \times \boldsymbol{r}\right)}{r^{3}}
$$

where $\mathrm{d}^{2} \boldsymbol{F}$ is the double differential of the force $\boldsymbol{F}$. These equations, on which the SI is based, are different from those used in the CGS-ESU, CGS-EMU, and CGSGaussian systems, where $\varepsilon_{0}$ and $\mu_{0}$ are dimensionless quantities, chosen to be equal to one, and where the rationalizing factors of $4 \pi$ are omitted.

\subsection{Dimensions of quantities}

By convention physical quantities are organized in a system of dimensions. Each of the seven base quantities used in the SI is regarded as having its own dimension, which is symbolically represented by a single sans serif roman capital letter. The symbols used for the base quantities, and the symbols used to denote their dimension, are given as follows.

Base quantities and dimensions used in the SI

Quantity symbols are always written in an italic font, and symbols for dimensions in sans-serif roman capitals.

For some quantities a variety of alternative symbols may be used, as indicated for length and electric current. Note that symbols for quantities are only recommendations, in contrast to symbols for units that appear elsewhere in this brochure whose style and

All other quantities are derived quantities, which may be written in terms of the base quantities by the equations of physics. The dimensions of the derived quantities are written as products of powers of the dimensions of the base quantities using the equations that relate the derived quantities to the base quantities. In general the dimension of any quantity $Q$ is written in the form of a dimensional product,

$$
\operatorname{dim} Q=\mathrm{L}^{\alpha} \mathrm{M}^{\beta} \mathrm{T}^{\gamma} \mathrm{I}^{\delta} \Theta^{\varepsilon} \mathbf{N}^{\zeta} \mathrm{J}^{\eta}
$$

where the exponents $\alpha, \beta, \gamma, \delta, \varepsilon, \zeta$, and $\eta$, which are generally small integers which can be positive, negative or zero, are called the dimensional exponents. The dimension of a derived quantity provides the same information about the relation of that quantity to the base quantities as is provided by the SI unit of the derived quantity as a product of powers of the SI base units.

There are some derived quantities $Q$ for which the defining equation is such that all of the dimensional exponents in the expression for the dimension of $Q$ are zero. This is true, in particular, for any quantity that is defined as the ratio of two quantities of the same kind. Such quantities are described as being dimensionless, or alternatively as being of dimension one. The coherent derived unit for such dimensionless quantities is always the number one, 1 , since it is the ratio of two identical units for two quantities of the same kind.

There are also some quantities that cannot be described in terms of the seven base quantities of the SI at all, but have the nature of a count. Examples are number of form is mandatory (see Chapter 5).

Dimensional symbols and exponents are manipulated using the ordinary rules of algebra. For example, the dimension of area is written as $\mathrm{L}^{2}$; the dimension of velocity as $\mathrm{LT}^{-1}$; the $\mathrm{LMT}^{-2}$; and the dimension of energy is written as $\mathrm{L}^{2} \mathrm{MT}^{-2}$.

For example, refractive index is defined as the ratio of the speed of light in vacuum to that in the medium, and is thus a ratio of two quantities of the same kind. It is therefore a dimensionless quantity. Other examples of dimensionless quantities are plane angle, mass fraction, relative permittivity, relative permeability, and finesse of a Fabry-Perot cavity. dimension of force as 
molecules, degeneracy in quantum mechanics (the number of independent states of the same energy), and the partition function in statistical thermodynamics (the number of thermally accessible states). Such counting quantities are also usually regarded as dimensionless quantities, or quantities of dimension one, with the unit one, 1 .

\subsection{Coherent units, derived units with special names, and the SI prefixes}

Derived units are defined as products of powers of the base units. When the product of powers includes no numerical factor other than one, the derived units are called coherent derived units. The base and coherent derived units of the SI form a coherent set, designated the set of coherent SI units. The word coherent is used here in the following sense: when coherent units are used, equations between the numerical values of quantities take exactly the same form as the equations between the quantities themselves. Thus if only units from a coherent set are used, conversion factors between units are never required.

The expression for the coherent unit of a derived quantity may be obtained from the dimensional product of that quantity by replacing the symbol for each dimension by the symbol of the corresponding base unit.

Some of the coherent derived units in the SI are given special names, to simplify their expression (see 2.2.2, p. 118). It is important to emphasize that each physical quantity has only one coherent SI unit, even if this unit can be expressed in different forms by using some of the special names and symbols. The inverse, however, is not true: in some cases the same SI unit can be used to express the values of several different quantities (see p. 119).

The CGPM has, in addition, adopted a series of prefixes for use in forming the decimal multiples and submultiples of the coherent SI units (see 3.1, p. 121, where the prefix names and symbols are listed). These are convenient for expressing the values of quantities that are much larger than or much smaller than the coherent unit. Following the CIPM Recommendation 1 (1969) (see p. 155) these are given the name SI Prefixes. (These prefixes are also sometimes used with other non-SI units, as described in Chapter 4 below.) However when prefixes are used with SI units, the resulting units are no longer coherent, because a prefix on a derived unit effectively introduces a numerical factor in the expression for the derived unit in terms of the base units.

As an exception, the name of the kilogram, which is the base unit of mass, includes the prefix kilo, for historical reasons. It is nonetheless taken to be a base unit of the SI. The multiples and submultiples of the kilogram are formed by attaching prefix names to the unit name "gram", and prefix symbols to the unit symbol "g" (see 3.2, p. 122). Thus $10^{-6} \mathrm{~kg}$ is written as a milligram, $\mathrm{mg}$, not as a microkilogram, $\mu \mathrm{kg}$.

The complete set of SI units, including both the coherent set and the multiples and submultiples of these units formed by combining them with the SI prefixes, are designated as the complete set of SI units, or simply the SI units, or the units of the SI. Note, however, that the decimal multiples and submultiples of the SI units do not form a coherent set.

As an example of a special name, the particular combination of base units $\mathrm{m}^{2} \mathrm{~kg} \mathrm{~s}^{-2}$ for energy is given the special name joule, symbol J, where by definition $\mathrm{J}=\mathrm{m}^{2} \mathrm{~kg} \mathrm{~s}^{-2}$.

The length of a chemical bond is more conveniently given in nanometres, $\mathrm{nm}$, than in metres, $\mathrm{m}$; and the distance from London to Paris is more conveniently given in kilometres, $\mathrm{km}$, than in metres, $\mathrm{m}$.

The metre per second, symbol $\mathrm{m} / \mathrm{s}$, is the coherent SI unit of speed. The kilometre per second, $\mathrm{km} / \mathrm{s}$, the centimetre per second, $\mathrm{cm} / \mathrm{s}$, and the millimetre per second, $\mathrm{mm} / \mathrm{s}$, are also SI units, but they are not coherent SI units. 


\subsection{SI units in the framework of general relativity}

The definitions of the base units of the SI were adopted in a context that takes no account of relativistic effects. When such account is taken, it is clear that the definitions apply only in a small spatial domain sharing the motion of the standards that realize them. These units are known as proper units; they are realized from local experiments in which the relativistic effects that need to be taken into account are those of special relativity. The constants of physics are local quantities with their values expressed in proper units.

Physical realizations of the definition of a unit are usually compared locally. For frequency standards, however, it is possible to make such comparisons at a distance by means of electromagnetic signals. To interpret the results the theory of general relativity is required since it predicts, among other things, a relative frequency shift between standards of about 1 part in $10^{16}$ per metre of altitude difference at the surface of the Earth. Effects of this magnitude cannot be neglected when comparing the best frequency standards.

\subsection{Units for quantities that describe biological effects}

Units for quantities that describe biological effects are often difficult to relate to units of the SI because they typically involve weighting factors that may not be precisely known or defined, and which may be both energy and frequency dependent. These units, which are not SI units, are described briefly in this section.

Optical radiation may cause chemical changes in living or non-living materials: this property is called actinism and radiation capable of causing such changes is referred to as actinic radiation. In some cases, the results of measurements of photochemical and photobiological quantities of this kind can be expressed in terms of SI units. This is discussed briefly in Appendix 3.

Sound causes small pressure fluctuations in the air, superimposed on the normal atmospheric pressure, that are sensed by the human ear. The sensitivity of the ear depends on the frequency of the sound, but is not a simple function of either the pressure changes or the frequency. Therefore frequency-weighted quantities are used in acoustics to approximate the way in which sound is perceived. Such frequencyweighted quantities are employed, for example, in work to protect against hearing damage. The effects of ultrasonic acoustic waves pose similar concerns in medical diagnosis and therapy.

Ionizing radiation deposits energy in irradiated matter. The ratio of deposited energy to mass is termed absorbed dose. High doses of ionizing radiation kill cells, and this is used in radiation therapy. Appropriate biological weighting functions are used to compare therapeutic effects of different radiation treatments. Low sub-lethal doses can cause damage to living organisms, for instance by inducing cancer. Appropriate risk-weighted functions are used at low doses as the basis of radiation protection regulations.

There is a class of units for quantifying the biological activity of certain substances used in medical diagnosis and therapy that cannot yet be defined in terms of the units of the SI. This is because the mechanism of the specific biological effect that gives these substances their medical use is not yet sufficiently well understood for it to be quantifiable in terms of physico-chemical parameters. In view of their importance for
The question of proper units is addressed in Resolution

A4 adopted by the XXIst General Assembly of the International Astronomical Union (IAU) in 1991 and by the report of the CCDS Working Group on the Application of General Relativity to Metrology (Metrologia, 1997, 34, 261-290). 
human health and safety, the World Health Organization (WHO) has taken responsibility for defining WHO International Units (IU) for the biological activity of such substances.

\subsection{Legislation on units}

By legislation, individual countries have established rules concerning the use of units on a national basis, either for general use or for specific areas such as commerce, health, public safety, and education. In almost all countries this legislation is based on the International System of Units.

The Organisation Internationale de Métrologie Légale (OIML), founded in 1955, is charged with the international harmonization of this legislation.

\section{$1.8 \quad$ Historical note}

The previous paragraphs of this chapter give a brief overview of the way in which a system of units, and the International System of Units in particular, is established. This note gives a brief account of the historical development of the International System.

The 9th CGPM (1948, Resolution 6; CR, 64) instructed the CIPM:

- to study the establishment of a complete set of rules for units of measurement;

- to find out for this purpose, by official enquiry, the opinion prevailing in scientific, technical and educational circles in all countries;

- to make recommendations on the establishment of a practical system of units of measurement suitable for adoption by all signatories to the Convention du Mètre.

The same CGPM also laid down, in Resolution 7 (CR, 70), general principles for the writing of unit symbols, and listed some coherent derived units which were assigned special names.

The 10th CGPM (1954, Resolution 6; CR, 80) and the 14th CGPM (1971, Resolution 3, CR, 78, and Metrologia, 1972, 8, 36) adopted as base units of this practical system of units the units of the following seven quantities: length, mass, time, electric current, thermodynamic temperature, amount of substance, and luminous intensity.

The 11th CGPM (1960, Resolution 12; CR, 87) adopted the name Système International d'Unités, with the international abbreviation SI, for this practical system of units and laid down rules for prefixes, derived units, and the former supplementary units, and other matters; it thus established a comprehensive specification for units of measurement. Subsequent meetings of the CGPM and CIPM have added to, and modified as necessary, the original structure of the SI to take account of advances in science and of the needs of users.

The historical sequence that led to these important CGPM decisions may be summarized as follows.

- The creation of the decimal metric system at the time of the French Revolution and the subsequent deposition of two platinum standards representing the metre and the kilogram, on 22 June 1799, in the Archives de la République in Paris can 
be seen as the first step in the development of the present International System of Units.

- In 1832, Gauss strongly promoted the application of this metric system, together with the second defined in astronomy, as a coherent system of units for the physical sciences. Gauss was the first to make absolute measurements of the Earth's magnetic field in terms of a decimal system based on the three mechanical units millimetre, gram, and second for, respectively, the quantities length, mass, and time. In later years, Gauss and Weber extended these measurements to include other electrical phenomena.

- These applications in the field of electricity and magnetism were further developed in the 1860s under the active leadership of Maxwell and Thomson through the British Association for the Advancement of Science (BAAS). They formulated the requirement for a coherent system of units with base units and derived units. In 1874 the BAAS introduced the CGS system, a three-dimensional coherent unit system based on the three mechanical units centimetre, gram, and second, using prefixes ranging from micro to mega to express decimal submultiples and multiples. The subsequent development of physics as an experimental science was largely based on this system.

- The sizes of the coherent CGS units in the fields of electricity and magnetism proved to be inconvenient so, in the 1880s, the BAAS and the International Electrical Congress, predecessor of the International Electrotechnical Commission (IEC), approved a mutually coherent set of practical units. Among them were the ohm for electrical resistance, the volt for electromotive force, and the ampere for electric current.

- After the signing of the Convention du Mètre on 20 May 1875, which created the BIPM and established the CGPM and the CIPM, work began on the construction of new international prototypes of the metre and kilogram. In 1889 the first CGPM sanctioned the international prototypes for the metre and the kilogram. Together with the astronomical second as the unit of time, these units constituted a three-dimensional mechanical unit system similar to the CGS system, but with the base units metre, kilogram, and second, the MKS system.

- In 1901 Giorgi showed that it is possible to combine the mechanical units of this metre-kilogram-second system with the practical electrical units to form a single coherent four-dimensional system by adding to the three base units a fourth unit, of an electrical nature such as the ampere or the ohm, and rewriting the equations occurring in electromagnetism in the so-called rationalized form. Giorgi's proposal opened the path to a number of new developments.

- After the revision of the Convention du Mètre by the 6th CGPM in 1921, which extended the scope and responsibilities of the BIPM to other fields in physics, and the subsequent creation of the Consultative Committee for Electricity (CCE) by the 7th CGPM in 1927, the Giorgi proposal was thoroughly discussed by the IEC, the International Union of Pure and Applied Physics (IUPAP), and other international organizations. This led the CCE to propose, in 1939, the adoption of a four-dimensional system based on the metre, kilogram, second, and ampere, the MKSA system, a proposal approved by the CIPM in 1946. 
- Following an international enquiry by the BIPM, which began in 1948, the 10th CGPM, in 1954, approved the introduction of the ampere, the kelvin, and the candela as base units, respectively, for electric current, thermodynamic temperature, and luminous intensity. The name Système International d'Unités, with the abbreviation SI, was given to the system by the 11th CGPM in 1960. At the 14th CGPM in 1971, after lengthy discussions between physicists and chemists, the current version of the SI was completed by adding the mole as the base unit for amount of substance, bringing the total number of base units to seven. 


\section{SI units}

\section{$2.1 \quad$ SI base units}

Formal definitions of all SI base units are adopted by the CGPM. The first two definitions were adopted in 1889, and the most recent in 1983. These definitions are modified from time to time as science advances.

\subsubsection{Definitions}

Current definitions of the base units, as taken from the Comptes Rendus (CR) of the corresponding CGPM, are shown below indented and in a heavy sans-serif font. Related decisions which clarify these definitions but are not formally part of them, as taken from the Comptes Rendus of the corresponding CGPM or the Procès-Verbaux (PV) of the CIPM, are also shown indented but in a sans-serif font of normal weight. The linking text provides historical notes and explanations, but is not part of the definitions themselves.

It is important to distinguish between the definition of a unit and its realization. The definition of each base unit of the SI is carefully drawn up so that it is unique and provides a sound theoretical basis upon which the most accurate and reproducible measurements can be made. The realization of the definition of a unit is the procedure by which the definition may be used to establish the value and associated uncertainty of a quantity of the same kind as the unit. A description of how the definitions of some important units are realized in practice is given on the BIPM website,

\section{www.bipm.org/en/si/si brochure/appendix $2 /$.}

A coherent SI derived unit is defined uniquely only in terms of SI base units. For example, the coherent SI derived unit of resistance, the ohm, symbol $\Omega$, is uniquely defined by the relation $\Omega=\mathrm{m}^{2} \mathrm{~kg} \mathrm{~s}^{-3} \mathrm{~A}^{-2}$, which follows from the definition of the quantity electrical resistance. However any method consistent with the laws of physics could be used to realize any SI unit. For example, the unit ohm can be realized with high accuracy using the quantum Hall effect and the value of the von Klitzing constant recommended by the CIPM (see pp. 163 and 166, respectively, Appendix 1).

Finally, it should be recognized that although the seven base quantities - length, mass, time, electric current, thermodynamic temperature, amount of substance, and luminous intensity - are by convention regarded as independent, their respective base units - the metre, kilogram, second, ampere, kelvin, mole, and candela - are in a number of instances interdependent. Thus the definition of the metre incorporates the second; the definition of the ampere incorporates the metre, kilogram, and second; the definition of the mole incorporates the kilogram; and the definition of the candela incorporates the metre, kilogram, and second. 


\subsubsection{Unit of length (metre)}

The 1889 definition of the metre, based on the international prototype of platinumiridium, was replaced by the 11th CGPM (1960) using a definition based on the wavelength of krypton 86 radiation. This change was adopted in order to improve the accuracy with which the definition of the metre could be realized, the realization being achieved using an interferometer with a travelling microscope to measure the optical path difference as the fringes were counted. In turn, this was replaced in 1983 by the 17th CGPM (1983, Resolution 1, CR, 97, and Metrologia, 1984, 20, 25) that specified the current definition, as follows:

The metre is the length of the path travelled by light in vacuum during a time interval of $1 / 299792458$ of a second.

It follows that the speed of light in vacuum is exactly 299792458 metres per second, $c_{0}=299792458 \mathrm{~m} / \mathrm{s}$.

The original international prototype of the metre, which was sanctioned by the 1st CGPM in 1889 (CR, 34-38), is still kept at the BIPM under conditions specified in 1889 .

\subsubsection{Unit of mass (kilogram)}

The international prototype of the kilogram, an artefact made of platinum-iridium, is kept at the BIPM under the conditions specified by the 1st CGPM in 1889 (CR, 3438) when it sanctioned the prototype and declared:

This prototype shall henceforth be considered to be the unit of mass.

The 3rd CGPM (1901, CR, 70), in a declaration intended to end the ambiguity in popular usage concerning the use of the word "weight", confirmed that:

The kilogram is the unit of mass; it is equal to the mass of the international prototype of the kilogram.

The complete declaration appears on p. 143.

It follows that the mass of the international prototype of the kilogram is always 1 kilogram exactly, $m(\mathcal{K})=1 \mathrm{~kg}$. However, due to the inevitable accumulation of contaminants on surfaces, the international prototype is subject to reversible surface contamination that approaches $1 \mu \mathrm{g}$ per year in mass. For this reason, the CIPM declared that, pending further research, the reference mass of the international prototype is that immediately after cleaning and washing by a specified method (PV, 1989, 57, 104-105 and PV, 1990, 58, 95-97). The reference mass thus defined is used to calibrate national standards of platinum-iridium alloy (Metrologia, 1994, 31, 317 336).

\subsubsection{Unit of time (second)}

The unit of time, the second, was at one time considered to be the fraction 1/86 400 of the mean solar day. The exact definition of "mean solar day" was left to the astronomers. However measurements showed that irregularities in the rotation of the Earth made this an unsatisfactory definition. In order to define the unit of time more precisely, the 11th CGPM (1960, Resolution 9; CR, 86) adopted a definition given by the International Astronomical Union based on the tropical year 1900. Experimental work, however, had already shown that an atomic standard of time, based on a transition between two energy levels of an atom or a molecule, could be realized and
The symbol, $c_{0}$ (or sometimes simply $c$ ), is the conventional symbol for the speed of light in vacuum.
The symbol, $m(\mathcal{K})$, is used to denote the mass of the international prototype of the kilogram, $\mathcal{K}$. 
reproduced much more accurately. Considering that a very precise definition of the unit of time is indispensable for science and technology, the 13th CGPM (1967/68, Resolution 1; CR, 103 and Metrologia, 1968, 4, 43) replaced the definition of the second by the following:

The second is the duration of 9192631770 periods of the radiation corresponding to the transition between the two hyperfine levels of the ground state of the caesium 133 atom.

It follows that the hyperfine splitting in the ground state of the caesium 133 atom is exactly 9192631770 hertz, $v($ hfs Cs $)=9192631770 \mathrm{~Hz}$.

At its 1997 meeting the CIPM affirmed that:

This definition refers to a caesium atom at rest at a temperature of $0 \mathrm{~K}$.

This note was intended to make it clear that the definition of the SI second is based on a caesium atom unperturbed by black body radiation, that is, in an environment whose thermodynamic temperature is $0 \mathrm{~K}$. The frequencies of all primary frequency standards should therefore be corrected for the shift due to ambient radiation, as stated at the meeting of the Consultative Committee for Time and Frequency in 1999.

\subsubsection{Unit of electric current (ampere)}

Electric units, called "international units", for current and resistance, were introduced by the International Electrical Congress held in Chicago in 1893, and definitions of the "international ampere" and "international ohm" were confirmed by the International Conference in London in 1908.

Although it was already obvious on the occasion of the 8th CGPM (1933) that there was a unanimous desire to replace those "international units" by so-called "absolute units", the official decision to abolish them was only taken by the 9th CGPM (1948), which adopted the ampere for the unit of electric current, following a definition proposed by the CIPM (1946, Resolution 2; PV, 20, 129-137):

The ampere is that constant current which, if maintained in two straight parallel conductors of infinite length, of negligible circular cross-section, and placed 1 metre apart in vacuum, would produce between these conductors a force equal to $2 \times 10^{-7}$ newton per metre of length.

It follows that the magnetic constant, $\mu_{0}$, also known as the permeability of free space, is exactly $4 \pi \times 10^{-7}$ henries per metre, $\mu_{0}=4 \pi \times 10^{-7} \mathrm{H} / \mathrm{m}$.

The expression "MKS unit of force" which occurs in the original text of 1946 has been replaced here by "newton", a name adopted for this unit by the 9th CGPM (1948, Resolution 7; CR, 70).

\subsubsection{Unit of thermodynamic temperature (kelvin)}

The definition of the unit of thermodynamic temperature was given in substance by the 10th CGPM (1954, Resolution 3; CR, 79) which selected the triple point of water as the fundamental fixed point and assigned to it the temperature $273.16 \mathrm{~K}$, so defining the unit. The 13th CGPM (1967/68, Resolution 3; CR, 104 and Metrologia, $1968,4,43$ ) adopted the name kelvin, symbol K, instead of "degree Kelvin", symbol ${ }^{\circ} \mathrm{K}$, and defined the unit of thermodynamic temperature as follows (1967/68, Resolution 4; CR, 104 and Metrologia, 1968, 4, 43):
The symbol, $v(\mathrm{hfs} \mathrm{Cs})$, is used to denote the frequency of the hyperfine transition in the ground state of the caesium atom. 


\section{The kelvin, unit of thermodynamic temperature, is the fraction $1 / 273.16$ of the thermodynamic temperature of the triple point of water.}

It follows that the thermodynamic temperature of the triple point of water is exactly 273.16 kelvins, $T_{\text {tpw }}=273.16 \mathrm{~K}$.

At its 2005 meeting the CIPM affirmed that:

This definition refers to water having the isotopic composition defined exactly by the following amount of substance ratios: 0.00015576 mole of ${ }^{2} \mathrm{H}$ per mole of ${ }^{1} \mathrm{H}, 0.0003799$ mole of ${ }^{17} \mathrm{O}$ per mole of ${ }^{16} \mathrm{O}$, and 0.0020052 mole of ${ }^{18} \mathrm{O}$ per mole of ${ }^{16} \mathrm{O}$.

Because of the manner in which temperature scales used to be defined, it remains common practice to express a thermodynamic temperature, symbol $T$, in terms of its difference from the reference temperature $T_{0}=273.15 \mathrm{~K}$, the ice point. This difference is called the Celsius temperature, symbol $t$, which is defined by the quantity equation:

$$
t=T-T_{0} .
$$

The unit of Celsius temperature is the degree Celsius, symbol ${ }^{\circ} \mathrm{C}$, which is by definition equal in magnitude to the kelvin. A difference or interval of temperature may be expressed in kelvins or in degrees Celsius (13th CGPM, 1967/68, Resolution 3 , mentioned above), the numerical value of the temperature difference being the same. However, the numerical value of a Celsius temperature expressed in degrees Celsius is related to the numerical value of the thermodynamic temperature expressed in kelvins by the relation

$$
t /{ }^{\circ} \mathrm{C}=T / \mathrm{K}-273.15 .
$$

The kelvin and the degree Celsius are also units of the International Temperature Scale of 1990 (ITS-90) adopted by the CIPM in 1989 in its Recommendation 5 (CI1989; PV, 57, 115 and Metrologia, 1990, 27, 13).

\subsubsection{Unit of amount of substance (mole)}

Following the discovery of the fundamental laws of chemistry, units called, for example, "gram-atom" and "gram-molecule", were used to specify amounts of chemical elements or compounds. These units had a direct connection with "atomic weights" and "molecular weights", which are in fact relative masses. "Atomic weights" were originally referred to the atomic weight of oxygen, by general agreement taken as 16. But whereas physicists separated the isotopes in a mass spectrometer and attributed the value 16 to one of the isotopes of oxygen, chemists attributed the same value to the (slightly variable) mixture of isotopes 16, 17 and 18, which was for them the naturally occurring element oxygen. Finally an agreement between the International Union of Pure and Applied Physics (IUPAP) and the International Union of Pure and Applied Chemistry (IUPAC) brought this duality to an end in 1959/60. Physicists and chemists have ever since agreed to assign the value 12 , exactly, to the so-called atomic weight of the isotope of carbon with mass number 12 (carbon $12,{ }^{12} \mathrm{C}$ ), correctly called the relative atomic mass $A_{\mathrm{r}}\left({ }^{12} \mathrm{C}\right.$ ). The unified scale thus obtained gives the relative atomic and molecular masses, also known as the atomic and molecular weights, respectively.

The quantity used by chemists to specify the amount of chemical elements or compounds is now called "amount of substance". Amount of substance is defined to be proportional to the number of specified elementary entities in a sample, the
The symbol, $T_{\mathrm{tpw}}$, is used to denote the thermodynamic temperature of the triple point of water.
The recommended symbol for relative atomic mass (atomic weight) is $A_{\mathrm{r}}(\mathrm{X})$, where the atomic entity $\mathrm{X}$ should be specified, and for relative molecular mass of a molecule (molecular weight) it is $M_{\mathrm{r}}(\mathrm{X})$, where the molecular entity $\mathrm{X}$ should be specified. 
proportionality constant being a universal constant which is the same for all samples. The unit of amount of substance is called the mole, symbol mol, and the mole is defined by specifying the mass of carbon 12 that constitutes one mole of carbon 12 atoms. By international agreement this was fixed at $0.012 \mathrm{~kg}$, i.e. $12 \mathrm{~g}$.

Following proposals by the IUPAP, the IUPAC, and the ISO, the CIPM gave a definition of the mole in 1967 and confirmed it in 1969. This was adopted by the 14th CGPM (1971, Resolution 3; CR, 78 and Metrologia, 1972, 8, 36):

1. The mole is the amount of substance of a system which contains as many elementary entities as there are atoms in 0.012 kilogram of carbon 12; its symbol is "mol".

2. When the mole is used, the elementary entities must be specified and may be atoms, molecules, ions, electrons, other particles, or specified groups of such particles.

It follows that the molar mass of carbon 12 is exactly 12 grams per mole, $M\left({ }^{12} \mathrm{C}\right)=12 \mathrm{~g} / \mathrm{mol}$.

In 1980 the CIPM approved the report of the CCU (1980) which specified that

In this definition, it is understood that unbound atoms of carbon 12, at rest and in their ground state, are referred to.

The definition of the mole also determines the value of the universal constant that relates the number of entities to amount of substance for any sample. This constant is called the Avogadro constant, symbol $N_{\mathrm{A}}$ or $L$. If $N(\mathrm{X})$ denotes the number of entities $\mathrm{X}$ in a specified sample, and if $n(\mathrm{X})$ denotes the amount of substance of entities $\mathrm{X}$ in the same sample, the relation is

$$
n(\mathrm{X})=N(\mathrm{X}) / N_{\mathrm{A}} \text {. }
$$

Note that since $N(\mathrm{X})$ is dimensionless, and $n(\mathrm{X})$ has the SI unit mole, the Avogadro constant has the coherent SI unit reciprocal mole.

In the name "amount of substance", the words "of substance" could for simplicity be replaced by words to specify the substance concerned in any particular application, so that one may, for example, talk of "amount of hydrogen chloride, $\mathrm{HCl}$ ", or "amount of benzene, $\mathrm{C}_{6} \mathrm{H}_{6}$ ". It is important to always give a precise specification of the entity involved (as emphasized in the second sentence of the definition of the mole); this should preferably be done by giving the empirical chemical formula of the material involved. Although the word "amount" has a more general dictionary definition, this abbreviation of the full name "amount of substance" may be used for brevity. This also applies to derived quantities such as "amount of substance concentration", which may simply be called "amount concentration". However, in the field of clinical chemistry the name "amount of substance concentration" is generally abbreviated to "substance concentration".

\subsubsection{Unit of luminous intensity (candela)}

The units of luminous intensity based on flame or incandescent filament standards in use in various countries before 1948 were replaced initially by the "new candle" based on the luminance of a Planck radiator (a black body) at the temperature of freezing platinum. This modification had been prepared by the International Commission on Illumination (CIE) and by the CIPM before 1937, and the decision was promulgated by the CIPM in 1946. It was then ratified in 1948 by the 9th CGPM which adopted a new international name for this unit, the candela, symbol cd; in 
1967 the 13th CGPM (Resolution 5, CR, 104 and Metrologia, 1968, 4, 43-44) gave an amended version of this definition.

In 1979, because of the difficulties in realizing a Planck radiator at high temperatures, and the new possibilities offered by radiometry, i.e. the measurement of optical radiation power, the 16th CGPM (1979, Resolution 3; CR, 100 and Metrologia, 1980, $\mathbf{1 6}, 56$ ) adopted a new definition of the candela:

The candela is the luminous intensity, in a given direction, of a source that emits monochromatic radiation of frequency $540 \times 10^{12}$ hertz and that has a radiant intensity in that direction of $1 / 683$ watt per steradian.

It follows that the spectral luminous efficacy for monochromatic radiation of frequency of $540 \times 10^{12}$ hertz is exactly 683 lumens per watt, $K=683 \mathrm{~lm} / \mathrm{W}$ $=683 \mathrm{~cd} \mathrm{sr} / \mathrm{W}$.

\subsubsection{Symbols for the seven base units}

The base units of the International System are listed in Table 1, which relates the base quantity to the unit name and unit symbol for each of the seven base units (10th CGPM (1954, Resolution 6; CR, 80); 11th CGPM (1960, Resolution 12; CR, 87); 13th CGPM (1967/68, Resolution 3; CR, 104 and Metrologia, 1968, 4, 43); 14th CGPM (1971, Resolution 3; CR, 78 and Metrologia, 1972, 8, 36)).

Table 1. SI base units

\begin{tabular}{|c|c|c|c|}
\hline \multicolumn{2}{|l|}{ Base quantity } & \multicolumn{2}{|c|}{ SI base unit } \\
\hline Name & Symbol & Name & Symbol \\
\hline length & $l, x, r$, etc. & metre & $\mathrm{m}$ \\
\hline mass & $m$ & kilogram & $\mathrm{kg}$ \\
\hline time, duration & $t$ & second & s \\
\hline electric current & $I, i$ & ampere & A \\
\hline thermodynamic temperature & $T$ & kelvin & $\mathrm{K}$ \\
\hline amount of substance & $n$ & mole & mol \\
\hline luminous intensity & $I_{\mathrm{V}}$ & candela & $\mathrm{cd}$ \\
\hline
\end{tabular}

The symbols for quantities are generally single letters of the Latin or Greek alphabets, printed in an italic font, and are recommendations.

The symbols for units are mandatory, see chapter 5 .

\section{$2.2 \quad$ SI derived units}

Derived units are products of powers of base units. Coherent derived units are products of powers of base units that include no numerical factor other than 1 . The base and coherent derived units of the SI form a coherent set, designated the set of coherent SI units (see 1.4, p. 106).

\subsubsection{Derived units expressed in terms of base units}

The number of quantities in science is without limit, and it is not possible to provide a complete list of derived quantities and derived units. However, Table 2 lists some examples of derived quantities, and the corresponding coherent derived units expressed directly in terms of base units. 
Table 2. Examples of coherent derived units in the SI expressed in terms of base units

\begin{tabular}{|c|c|c|c|}
\hline \multicolumn{2}{|l|}{ Derived quantity } & \multicolumn{2}{|l|}{ SI coherent derived unit } \\
\hline Name & Symbol & Name & Symbol \\
\hline area & $A$ & square metre & $\mathrm{m}^{2}$ \\
\hline volume & $V$ & cubic metre & $\mathrm{m}^{3}$ \\
\hline speed, velocity & $v$ & metre per second & $\mathrm{m} / \mathrm{s}$ \\
\hline acceleration & $a$ & metre per second squared & $\mathrm{m} / \mathrm{s}^{2}$ \\
\hline wavenumber & $\sigma, \widetilde{v}$ & reciprocal metre & $\mathrm{m}^{-1}$ \\
\hline density, mass density & $\rho$ & kilogram per cubic metre & $\mathrm{kg} / \mathrm{m}^{3}$ \\
\hline surface density & $\rho_{\mathrm{A}}$ & kilogram per square metre & $\mathrm{kg} / \mathrm{m}^{2}$ \\
\hline specific volume & $v$ & cubic metre per kilogram & $\mathrm{m}^{3} / \mathrm{kg}$ \\
\hline current density & $j$ & ampere per square metre & $\mathrm{A} / \mathrm{m}^{2}$ \\
\hline magnetic field strength & $H$ & ampere per metre & $\mathrm{A} / \mathrm{m}$ \\
\hline $\begin{array}{l}\text { amount concentration }{ }^{(a)} \text {, } \\
\text { concentration }\end{array}$ & $c$ & mole per cubic metre & $\mathrm{mol} / \mathrm{m}^{3}$ \\
\hline mass concentration & $\rho, \gamma$ & kilogram per cubic metre & $\mathrm{kg} / \mathrm{m}^{3}$ \\
\hline luminance & $L_{\mathrm{V}}$ & candela per square metre & $\mathrm{cd} / \mathrm{m}^{2}$ \\
\hline refractive index ${ }^{(b)}$ & $n$ & one & 1 \\
\hline relative permeability ${ }^{(b)}$ & $\mu_{\mathrm{r}}$ & one & 1 \\
\hline \multicolumn{4}{|c|}{ (a) In the field of clinical chemistry this quantity is also called substance concentration. } \\
\hline \multicolumn{4}{|c|}{$\begin{array}{l}\text { (b) These are dimensionless quantities, or quantities of dimension one, and the symbol " } 1 \text { " for the } \\
\text { unit (the number "one") is generally omitted in specifying the values of dimensionles } \\
\text { quantities. }\end{array}$} \\
\hline
\end{tabular}

\subsubsection{Units with special names and symbols; units that incorporate special names and symbols}

For convenience, certain coherent derived units have been given special names and symbols. There are 22 such units, as listed in Table 3. These special names and symbols may themselves be used in combination with the names and symbols for base units and for other derived units to express the units of other derived quantities. Some examples are given in Table 4 . The special names and symbols are simply a compact form for the expression of combinations of base units that are used frequently, but in many cases they also serve to remind the reader of the quantity involved. The SI prefixes may be used with any of the special names and symbols, but when this is done the resulting unit will no longer be coherent.

Among these names and symbols the last four entries in Table 3 are of particular note since they were adopted by the 15th CGPM (1975, Resolutions 8 and 9; CR, 105 and Metrologia, 1975, 11, 180), the 16th CGPM (1979, Resolution 5; CR, 100 and Metrologia, 1980, 16, 56) and the 21st CGPM (1999, Resolution 12; CR, 334-335 and Metrologia, 2000, 37, 95) specifically with a view to safeguarding human health.

In both Tables 3 and 4 the final column shows how the SI units concerned may be expressed in terms of SI base units. In this column factors such as $\mathrm{m}^{0}, \mathrm{~kg}^{0}$, etc., which are all equal to 1 , are not shown explicitly. 
Table 3. Coherent derived units in the SI with special names and symbols

\begin{tabular}{|c|c|c|c|c|}
\hline \multirow[b]{2}{*}{ Derived quantity } & \multicolumn{4}{|c|}{ SI coherent derived unit ${ }^{(a)}$} \\
\hline & Name & Symbol & $\begin{array}{l}\text { Expressed } \\
\text { in terms of } \\
\text { other SI units }\end{array}$ & $\begin{array}{l}\text { Expressed } \\
\text { in terms of } \\
\text { SI base units }\end{array}$ \\
\hline plane angle & radian $^{(b)}$ & $\mathrm{rad}$ & $1^{(b)}$ & $\mathrm{m} / \mathrm{m}$ \\
\hline solid angle & steradian $^{(b)}$ & $\mathrm{sr}^{(c)}$ & $1^{(b)}$ & $\mathrm{m}^{2} / \mathrm{m}^{2}$ \\
\hline frequency & hertz $^{(d)}$ & $\mathrm{Hz}$ & & $\mathrm{s}^{-1}$ \\
\hline force & newton & $\mathrm{N}$ & & $\mathrm{m} \mathrm{kg} \mathrm{s}^{-2}$ \\
\hline pressure, stress & pascal & $\mathrm{Pa}$ & $\mathrm{N} / \mathrm{m}^{2}$ & $\mathrm{~m}^{-1} \mathrm{~kg} \mathrm{~s}^{-2}$ \\
\hline $\begin{array}{l}\text { energy, work, } \\
\text { amount of heat }\end{array}$ & joule & $\mathrm{J}$ & $\mathrm{N} \mathrm{m}$ & $\mathrm{m}^{2} \mathrm{~kg} \mathrm{~s}^{-2}$ \\
\hline power, radiant flux & watt & W & $\mathrm{J} / \mathrm{s}$ & $\mathrm{m}^{2} \mathrm{~kg} \mathrm{~s}^{-3}$ \\
\hline $\begin{array}{l}\text { electric charge, } \\
\text { amount of electricity }\end{array}$ & coulomb & $\mathrm{C}$ & & s A \\
\hline $\begin{array}{l}\text { electric potential difference, } \\
\text { electromotive force }\end{array}$ & volt & $\mathrm{V}$ & W/A & $\mathrm{m}^{2} \mathrm{~kg} \mathrm{~s}^{-3} \mathrm{~A}^{-1}$ \\
\hline capacitance & farad & $\mathrm{F}$ & $\mathrm{C} / \mathrm{V}$ & $\mathrm{m}^{-2} \mathrm{~kg}^{-1} \mathrm{~s}^{4} \mathrm{~A}^{2}$ \\
\hline electric resistance & ohm & $\Omega$ & $\mathrm{V} / \mathrm{A}$ & $\mathrm{m}^{2} \mathrm{~kg} \mathrm{~s}^{-3} \mathrm{~A}^{-2}$ \\
\hline electric conductance & siemens & S & $\mathrm{A} / \mathrm{V}$ & $\mathrm{m}^{-2} \mathrm{~kg}^{-1} \mathrm{~s}^{3} \mathrm{~A}^{2}$ \\
\hline magnetic flux & weber & $\mathrm{Wb}$ & V s & $\mathrm{m}^{2} \mathrm{~kg} \mathrm{~s}^{-2} \mathrm{~A}^{-1}$ \\
\hline magnetic flux density & tesla & $\mathrm{T}$ & $\mathrm{Wb} / \mathrm{m}^{2}$ & $\mathrm{~kg} \mathrm{~s}^{-2} \mathrm{~A}^{-1}$ \\
\hline inductance & henry & $\mathrm{H}$ & $\mathrm{Wb} / \mathrm{A}$ & $\mathrm{m}^{2} \mathrm{~kg} \mathrm{~s}^{-2} \mathrm{~A}^{-2}$ \\
\hline Celsius temperature & degree Celsius $^{(e)}$ & ${ }^{\circ} \mathrm{C}$ & & $\mathrm{K}$ \\
\hline luminous flux & lumen & $\operatorname{lm}$ & $\mathrm{cd} \mathrm{sr}^{(c)}$ & $\mathrm{cd}$ \\
\hline illuminance & lux & lx & $\operatorname{lm} / \mathrm{m}^{2}$ & $\mathrm{~m}^{-2} \mathrm{~cd}$ \\
\hline $\begin{array}{l}\text { activity referred to } \\
\text { a radionuclide }\end{array}$ & becquerel ${ }^{(d)}$ & $\mathrm{Bq}$ & & $\mathrm{s}^{-1}$ \\
\hline $\begin{array}{l}\text { absorbed dose, } \\
\text { specific energy (imparted), } \\
\text { kerma }\end{array}$ & gray & Gy & $\mathrm{J} / \mathrm{kg}$ & $\mathrm{m}^{2} \mathrm{~s}^{-2}$ \\
\hline $\begin{array}{l}\text { dose equivalent, } \\
\text { ambient dose equivalent, } \\
\text { directional dose equivalent, } \\
\text { personal dose equivalent }\end{array}$ & sievert $^{(g)}$ & $\mathrm{Sv}$ & $\mathrm{J} / \mathrm{kg}$ & $\mathrm{m}^{2} \mathrm{~s}^{-2}$ \\
\hline catalytic activity & katal & kat & & $\mathrm{s}^{-1} \mathrm{~mol}$ \\
\hline
\end{tabular}

(a) The SI prefixes may be used with any of the special names and symbols, but when this is done the resulting unit will no longer be coherent.

(b) The radian and steradian are special names for the number one that may be used to convey information about the quantity concerned. In practice the symbols rad and sr are used where appropriate, but the symbol for the derived unit one is generally omitted in specifying the values of dimensionless quantities.

(c) In photometry the name steradian and the symbol sr are usually retained in expressions for units.

(d) The hertz is used only for periodic phenomena, and the becquerel is used only for stochastic processes in activity referred to a radionuclide.

(e) The degree Celsius is the special name for the kelvin used to express Celsius temperatures. The degree Celsius and the kelvin are equal in size, so that the numerical value of a temperature difference or temperature interval is the same when expressed in either degrees Celsius or in kelvins.

(f) Activity referred to a radionuclide is sometimes incorrectly called radioactivity.

(g) See CIPM Recommendation 2 (CI-2002), p. 168, on the use of the sievert (PV, 2002, 70, 205). 
Table 4. Examples of $S I$ coherent derived units whose names and symbols include SI coherent derived units with special names and symbols

\begin{tabular}{|c|c|c|c|}
\hline \multirow[b]{2}{*}{ Derived quantity } & \multicolumn{3}{|l|}{ SI coherent derived unit } \\
\hline & Name & Symbol & $\begin{array}{l}\text { Expressed in terms of } \\
\text { SI base units }\end{array}$ \\
\hline dynamic viscosity & pascal second & $\mathrm{Pas}$ & $\mathrm{m}^{-1} \mathrm{~kg} \mathrm{~s}^{-1}$ \\
\hline moment of force & newton metre & $\mathrm{N} \mathrm{m}$ & $\mathrm{m}^{2} \mathrm{~kg} \mathrm{~s}^{-2}$ \\
\hline surface tension & newton per metre & $\mathrm{N} / \mathrm{m}$ & $\mathrm{kg} \mathrm{s}^{-2}$ \\
\hline angular velocity & radian per second & $\mathrm{rad} / \mathrm{s}$ & $\mathrm{m} \mathrm{m}^{-1} \mathrm{~s}^{-1}=\mathrm{s}^{-1}$ \\
\hline angular acceleration & radian per second squared & $\mathrm{rad} / \mathrm{s}^{2}$ & $\mathrm{~m} \mathrm{~m}^{-1} \mathrm{~s}^{-2}=\mathrm{s}^{-2}$ \\
\hline $\begin{array}{l}\text { heat flux density, } \\
\text { irradiance }\end{array}$ & watt per square metre & $\mathrm{W} / \mathrm{m}^{2}$ & $\mathrm{~kg} \mathrm{~s}^{-3}$ \\
\hline heat capacity, entropy & joule per kelvin & $\mathrm{J} / \mathrm{K}$ & $\mathrm{m}^{2} \mathrm{~kg} \mathrm{~s}^{-2} \mathrm{~K}^{-1}$ \\
\hline $\begin{array}{l}\text { specific heat capacity, } \\
\text { specific entropy }\end{array}$ & joule per kilogram kelvin & $\mathrm{J} /(\operatorname{kg~K})$ & $\mathrm{m}^{2} \mathrm{~s}^{-2} \mathrm{~K}^{-1}$ \\
\hline specific energy & joule per kilogram & $\mathrm{J} / \mathrm{kg}$ & $\mathrm{m}^{2} \mathrm{~s}^{-2}$ \\
\hline thermal conductivity & watt per metre kelvin & $\mathrm{W} /(\mathrm{m} \mathrm{K})$ & $\mathrm{m} \mathrm{kg} \mathrm{s}^{-3} \mathrm{~K}^{-1}$ \\
\hline energy density & joule per cubic metre & $\mathrm{J} / \mathrm{m}^{3}$ & $\mathrm{~m}^{-1} \mathrm{~kg} \mathrm{~s}^{-2}$ \\
\hline electric field strength & volt per metre & $\mathrm{V} / \mathrm{m}$ & $\mathrm{m} \mathrm{kg} \mathrm{s}^{-3} \mathrm{~A}^{-1}$ \\
\hline electric charge density & coulomb per cubic metre & $\mathrm{C} / \mathrm{m}^{3}$ & $\mathrm{~m}^{-3} \mathrm{~s} \mathrm{~A}$ \\
\hline surface charge density & coulomb per square metre & $\mathrm{C} / \mathrm{m}^{2}$ & $\mathrm{~m}^{-2} \mathrm{sA}$ \\
\hline $\begin{array}{l}\text { electric flux density, } \\
\text { electric displacement }\end{array}$ & coulomb per square metre & $\mathrm{C} / \mathrm{m}^{2}$ & $\mathrm{~m}^{-2} \mathrm{~s} \mathrm{~A}$ \\
\hline permittivity & farad per metre & $\mathrm{F} / \mathrm{m}$ & $\mathrm{m}^{-3} \mathrm{~kg}^{-1} \mathrm{~s}^{4} \mathrm{~A}^{2}$ \\
\hline permeability & henry per metre & $\mathrm{H} / \mathrm{m}$ & $\mathrm{m} \mathrm{kg} \mathrm{s}^{-2} \mathrm{~A}^{-2}$ \\
\hline molar energy & joule per mole & $\mathrm{J} / \mathrm{mol}$ & $\mathrm{m}^{2} \mathrm{~kg} \mathrm{~s}^{-2} \mathrm{~mol}^{-1}$ \\
\hline $\begin{array}{l}\text { molar entropy, } \\
\text { molar heat capacity }\end{array}$ & joule per mole kelvin & $\mathrm{J} /(\mathrm{mol} \mathrm{K})$ & $\mathrm{m}^{2} \mathrm{~kg} \mathrm{~s}^{-2} \mathrm{~K}^{-1} \mathrm{~mol}^{-1}$ \\
\hline exposure (x- and $\gamma$-rays) & coulomb per kilogram & $\mathrm{C} / \mathrm{kg}$ & $\mathrm{kg}^{-1} \mathrm{~s} \mathrm{~A}$ \\
\hline absorbed dose rate & gray per second & $\mathrm{Gy} / \mathrm{s}$ & $\mathrm{m}^{2} \mathrm{~s}^{-3}$ \\
\hline radiant intensity & watt per steradian & $\mathrm{W} / \mathrm{sr}$ & $\mathrm{m}^{4} \mathrm{~m}^{-2} \mathrm{~kg} \mathrm{~s}^{-3}=\mathrm{m}^{2} \mathrm{~kg} \mathrm{~s}^{-3}$ \\
\hline radiance & watt per square metre steradian & $\mathrm{W} /\left(\mathrm{m}^{2} \mathrm{sr}\right)$ & $\mathrm{m}^{2} \mathrm{~m}^{-2} \mathrm{~kg} \mathrm{~s}^{-3}=\mathrm{kg} \mathrm{s}^{-3}$ \\
\hline $\begin{array}{l}\text { catalytic activity } \\
\text { concentration }\end{array}$ & katal per cubic metre & $\mathrm{kat} / \mathrm{m}^{3}$ & $\mathrm{~m}^{-3} \mathrm{~s}^{-1} \mathrm{~mol}$ \\
\hline
\end{tabular}

The values of several different quantities may be expressed using the same name and symbol for the SI unit. Thus for the quantity heat capacity as well as the quantity entropy, the SI unit is the joule per kelvin. Similarly for the base quantity electric current as well as the derived quantity magnetomotive force, the SI unit is the ampere. It is therefore important not to use the unit alone to specify the quantity. This applies not only to scientific and technical texts, but also, for example, to measuring instruments (i.e. an instrument read-out should indicate both the unit and the quantity measured).

A derived unit can often be expressed in different ways by combining base units with derived units having special names. Joule, for example, may formally be written newton metre, or kilogram metre squared per second squared. This, however, is an algebraic freedom to be governed by common sense physical considerations; in a given situation some forms may be more helpful than others.

In practice, with certain quantities, preference is given to the use of certain special unit names, or combinations of unit names, to facilitate the distinction between different quantities having the same dimension. When using this freedom, one may recall the process by which the quantity is defined. For example, the quantity torque 
may be thought of as the cross product of force and distance, suggesting the unit newton metre, or it may be thought of as energy per angle, suggesting the unit joule per radian. The SI unit of frequency is given as the hertz, implying the unit cycles per second; the SI unit of angular velocity is given as the radian per second; and the SI unit of activity is designated the becquerel, implying the unit counts per second. Although it would be formally correct to write all three of these units as the reciprocal second, the use of the different names emphasises the different nature of the quantities concerned. Using the unit radian per second for angular velocity, and hertz for frequency, also emphasizes that the numerical value of the angular velocity in radian per second is $2 \pi$ times the numerical value of the corresponding frequency in hertz.

In the field of ionizing radiation, the SI unit of activity is designated the becquerel rather than the reciprocal second, and the SI units of absorbed dose and dose equivalent are designated the gray and the sievert, respectively, rather than the joule per kilogram. The special names becquerel, gray, and sievert were specifically introduced because of the dangers to human health that might arise from mistakes involving the units reciprocal second and joule per kilogram, in case the latter units were incorrectly taken to identify the different quantities involved.

\subsubsection{Units for dimensionless quantities, also called quantities of dimension one}

Certain quantities are defined as the ratio of two quantities of the same kind, and are thus dimensionless, or have a dimension that may be expressed by the number one. The coherent SI unit of all such dimensionless quantities, or quantities of dimension one, is the number one, since the unit must be the ratio of two identical SI units. The values of all such quantities are simply expressed as numbers, and the unit one is not explicitly shown. Examples of such quantities are refractive index, relative permeability, and friction factor. There are also some quantities that are defined as a more complex product of simpler quantities in such a way that the product is dimensionless. Examples include the "characteristic numbers" like the Reynolds number $R e=\rho v l / \eta$, where $\rho$ is mass density, $\eta$ is dynamic viscosity, $v$ is speed, and $l$ is length. For all these cases the unit may be considered as the number one, which is a dimensionless derived unit.

Another class of dimensionless quantities are numbers that represent a count, such as a number of molecules, degeneracy (number of energy levels), and partition function in statistical thermodynamics (number of thermally accessible states). All of these counting quantities are also described as being dimensionless, or of dimension one, and are taken to have the SI unit one, although the unit of counting quantities cannot be described as a derived unit expressed in terms of the base units of the SI. For such quantities, the unit one may instead be regarded as a further base unit.

In a few cases, however, a special name is given to the unit one, in order to facilitate the identification of the quantity involved. This is the case for the radian and the steradian. The radian and steradian have been identified by the CGPM as special names for the coherent derived unit one, to be used to express values of plane angle and solid angle, respectively, and are therefore included in Table 3.
The CIPM, recognizing the particular importance of the health-related units, adopted a detailed text on the sievert for the 5 th edition of this Brochure: Recommendation 1 (CI-1984), adopted by the CIPM (PV, 1984, 52, 31 and Metrologia, 1985, 21, 90), and Recommendation 2 (CI-2002), adopted by the CIPM (PV, 70, 205), see pp. 161 and 168 , respectively. 


\section{Decimal multiples and submultiples of SI units}

\subsection{SI prefixes}

The 11th CGPM (1960, Resolution 12; CR, 87) adopted a series of prefix names and prefix symbols to form the names and symbols of the decimal multiples and submultiples of SI units, ranging from $10^{12}$ to $10^{-12}$. Prefixes for $10^{-15}$ and $10^{-18}$ were added by the 12th CGPM (1964, Resolution 8; CR, 94), for $10^{15}$ and $10^{18}$ by the 15th CGPM (1975, Resolution 10; CR, 106 and Metrologia, 1975, 11, 180-181), and for $10^{21}, 10^{24}, 10^{-21}$ and $10^{-24}$ by the 19th CGPM (1991, Resolution 4; CR, 185 and Metrologia, 1992, 29, 3). Table 5 lists all approved prefix names and symbols.

Table 5. SI prefixes

\begin{tabular}{llllll}
\hline Factor & Name & Symbol & Factor & Name & Symbol \\
\hline $10^{1}$ & deca & da & $10^{-1}$ & deci & $\mathrm{d}$ \\
$10^{2}$ & hecto & $\mathrm{h}$ & $10^{-2}$ & centi & $\mathrm{c}$ \\
$10^{3}$ & kilo & $\mathrm{k}$ & $10^{-3}$ & milli & $\mathrm{m}$ \\
$10^{6}$ & mega & $\mathrm{M}$ & $10^{-6}$ & micro & $\mu$ \\
$10^{9}$ & giga & $\mathrm{G}$ & $10^{-9}$ & nano & $\mathrm{n}$ \\
$10^{12}$ & tera & $\mathrm{T}$ & $10^{-12}$ & pico & $\mathrm{p}$ \\
$10^{15}$ & peta & $\mathrm{P}$ & $10^{-15}$ & femto & $\mathrm{f}$ \\
$10^{18}$ & exa & $\mathrm{E}$ & $10^{-18}$ & atto & $\mathrm{a}$ \\
$10^{21}$ & zetta & $\mathrm{Z}$ & $10^{-21}$ & zepto & $\mathrm{z}$ \\
$10^{24}$ & yotta & $\mathrm{Y}$ & $10^{-24}$ & yocto & $\mathrm{y}$ \\
& & & & & \\
\hline
\end{tabular}

Prefix symbols are printed in roman (upright) type, as are unit symbols, regardless of the type used in the surrounding text, and are attached to unit symbols without a space between the prefix symbol and the unit symbol. With the exception of da (deca), h (hecto), and $\mathrm{k}$ (kilo), all multiple prefix symbols are capital (upper case) letters, and all submultiple prefix symbols are lower case letters. All prefix names are printed in lower case letters, except at the beginning of a sentence.

The grouping formed by a prefix symbol attached to a unit symbol constitutes a new inseparable unit symbol (forming a multiple or submultiple of the unit concerned) that can be raised to a positive or negative power and that can be combined with other unit symbols to form compound unit symbols.

$$
\begin{array}{ll}
\text { Examples: } & 2.3 \mathrm{~cm}^{3}=2.3(\mathrm{~cm})^{3}=2.3\left(10^{-2} \mathrm{~m}\right)^{3}=2.3 \times 10^{-6} \mathrm{~m}^{3} \\
& 1 \mathrm{~cm}^{-1}=1(\mathrm{~cm})^{-1}=1\left(10^{-2} \mathrm{~m}\right)^{-1}=10^{2} \mathrm{~m}^{-1}=100 \mathrm{~m}^{-1} \\
& 1 \mathrm{~V} / \mathrm{cm}=(1 \mathrm{~V}) /\left(10^{-2} \mathrm{~m}\right)=10^{2} \mathrm{~V} / \mathrm{m}=100 \mathrm{~V} / \mathrm{m} \\
& 5000 \mathrm{us}^{-1}=5000(\mu \mathrm{s})^{-1}=5000\left(10^{-6} \mathrm{~s}\right)^{-1}=5 \times 10^{9} \mathrm{~s}^{-1}
\end{array}
$$

These SI prefixes refer strictly to powers of 10 . They should not be used to indicate powers of 2 (for example, one kilobit represents 1000 bits and not 1024 bits). The IEC has adopted prefixes for binary powers in the international standard IEC 60027-2: 2005, third edition, Letter symbols to be used in electrical technology-Part 2: Telecommunications and electronics. The names and symbols for the prefixes corresponding to $2^{10}, 2^{20}$, $2^{30}, 2^{40}, 2^{50}$, and $2^{60}$ are, respectively: kibi, Ki; mebi, Mi; gibi, Gi; tebi, Ti; pebi, $\mathrm{Pi}$; and exbi, Ei. Thus, for example, one kibibyte would be written: $1 \mathrm{KiB}=2^{10} \mathrm{~B}=1024 \mathrm{~B}$, where $\mathrm{B}$ denotes a byte. Although these prefixes are not part of the SI, they should be used in the field of information technology to avoid the incorrect usage of the SI prefixes.

Examples of the use of prefixes: pm (picometre) mmol (millimole) G $\Omega$ (gigaohm) $\mathrm{THz}$ (terahertz) 
Similarly prefix names are also inseparable from the unit names to which they are attached. Thus, for example, millimetre, micropascal, and meganewton are single words.

Compound prefix symbols, that is, prefix symbols formed by the juxtaposition of two or more prefix symbols, are not permitted. This rule also applies to compound prefix names.

Prefix symbols can neither stand alone nor be attached to the number 1, the symbol for the unit one. Similarly, prefix names cannot be attached to the name of the unit one, that is, to the word "one."

Prefix names and symbols are used with a number of non-SI units (see Chapter 5), but they are never used with the units of time: minute, min; hour, h; day, d. However astronomers use milliarcsecond, which they denote mas, and microarcsecond, $\mu$ as, which they use as units for measuring very small angles.

\subsection{The kilogram}

Among the base units of the International System, the kilogram is the only one whose name and symbol, for historical reasons, include a prefix. Names and symbols for decimal multiples and submultiples of the unit of mass are formed by attaching prefix $10^{-6} \mathrm{~kg}=1 \mathrm{mg}$ but not $1 \mu \mathrm{kg}$ names to the unit name "gram", and prefix symbols to the unit symbol "g" (CIPM (microkilogram) 1967, Recommendation 2; PV, 35, 29 and Metrologia, 1968, 4, 45). 


\section{$4 \quad$ Units outside the SI}

The International System of Units, the SI, is a system of units, adopted by the CGPM, which provides the internationally agreed reference in terms of which all other units are now defined. It is recommended for use throughout science, technology, engineering, and commerce. The SI base units, and the SI coherent derived units, including those with special names, have the important advantage of forming a coherent set, with the effect that unit conversions are not required when inserting particular values for quantities into quantity equations. Because the SI is the only system of units that is globally recognized, it also has a clear advantage for establishing a worldwide dialogue. Finally, it simplifies the teaching of science and technology to the next generation if everyone uses this system.

Nonetheless it is recognized that some non-SI units still appear in the scientific, technical and commercial literature, and will continue to be used for many years. Some non-SI units are of historical importance in the established literature. Other non-SI units, such as the units of time and angle, are so deeply embedded in the history and culture of the human race that they will continue to be used for the foreseeable future. Individual scientists should also have the freedom to sometimes use non-SI units for which they see a particular scientific advantage in their work. An example of this is the use of CGS-Gaussian units in electromagnetic theory applied to quantum electrodynamics and relativity. For these reasons it is helpful to list some of the more important non-SI units, as is done below. However, if these units are used it should be understood that the advantages of the SI are lost.

The inclusion of non-SI units in this text does not imply that the use of non-SI units is to be encouraged. For the reasons already stated SI units are generally to be preferred. It is also desirable to avoid combining non-SI units with units of the SI; in particular, the combination of non-SI units with the SI to form compound units should be restricted to special cases in order not to compromise the advantages of the SI. Finally, when any of the non-SI units in Tables 7, 8, and 9 are used, it is good practice to define the non-SI unit in terms of the corresponding SI unit.

\subsection{Non-SI units accepted for use with the SI, and units based on fundamental constants}

The CIPM (2004) has revised the classification of non-SI units from that in the previous (7th) edition of this Brochure. Table 6 gives non-SI units that are accepted for use with the International System by the CIPM, because they are widely used with the SI in matters of everyday life. Their use is expected to continue indefinitely, and each has an exact definition in terms of an SI unit. Tables 7, 8 and 9 contain units that are used only in special circumstances. The units in Table 7 are related to fundamental constants, and their values have to be determined experimentally. Tables 8 and 9 contain units that have exactly defined values in terms of SI units, and are used in particular circumstances to satisfy the needs of commercial, legal, or 
specialized scientific interests. It is likely that these units will continue to be used for many years. Many of these units are also important for the interpretation of older scientific texts. Each of the Tables 6, 7, 8 and 9 is discussed in turn below.

Table 6 includes the traditional units of time and angle. It also contains the hectare, the litre, and the tonne, which are all in common everyday use throughout the world, and which differ from the corresponding coherent SI unit by an integer power of ten. The SI prefixes are used with several of these units, but not with the units of time.

Table 6. Non-SI units accepted for use with the International System of Units

\begin{tabular}{|c|c|c|c|}
\hline Quantity & Name of unit & Symbol for unit & Value in SI units \\
\hline \multirow[t]{3}{*}{ time } & minute & $\min$ & $1 \mathrm{~min}=60 \mathrm{~s}$ \\
\hline & hour $^{(a)}$ & $\mathrm{h}$ & $1 \mathrm{~h}=60 \mathrm{~min}=3600 \mathrm{~s}$ \\
\hline & day & $\mathrm{d}$ & $1 \mathrm{~d}=24 \mathrm{~h}=86400 \mathrm{~s}$ \\
\hline \multirow[t]{3}{*}{ plane angle } & degree ${ }^{(b, c)}$ & o & $1^{\circ}=(\pi / 180) \mathrm{rad}$ \\
\hline & minute & ' & $1^{\prime}=(1 / 60)^{\circ}=(\pi / 10800) \mathrm{rad}$ \\
\hline & second $^{(d)}$ & " & $1^{\prime \prime}=(1 / 60)^{\prime}=(\pi / 648000) \mathrm{rad}$ \\
\hline area & hectare $^{(e)}$ & ha & $1 \mathrm{ha}=1 \mathrm{hm}^{2}=10^{4} \mathrm{~m}^{2}$ \\
\hline volume & litre $^{(f)}$ & $\mathrm{L}, 1$ & $1 \mathrm{~L}=1 \mathrm{l}=1 \mathrm{dm}^{3}=10^{3} \mathrm{~cm}^{3}=10^{-3} \mathrm{~m}^{3}$ \\
\hline mass & tonne $(g)$ & $\mathrm{t}$ & $1 \mathrm{t}=10^{3} \mathrm{~kg}$ \\
\hline
\end{tabular}

(a) The symbol for this unit is included in Resolution 7 of the 9th CGPM (1948; CR, 70).

(b) ISO 31 recommends that the degree be divided decimally rather than using the minute and the second. For navigation and surveying, however, the minute has the advantage that one minute of latitude on the surface of the Earth corresponds (approximately) to one nautical mile.

(c) The gon (or grad, where grad is an alternative name for the gon) is an alternative unit of plane angle to the degree, defined as $(\pi / 200)$ rad. Thus there are 100 gon in a right angle. The potential value of the gon in navigation is that because the distance from the pole to the equator of the Earth is approximately $10000 \mathrm{~km}, 1 \mathrm{~km}$ on the surface of the Earth subtends an angle of one centigon at the centre of the Earth. However the gon is rarely used.

(d) For applications in astronomy, small angles are measured in arcseconds (i.e. seconds of plane angle), denoted as or ", milliarcseconds, microarcseconds, and picoarcseconds, denoted mas, $\mu \mathrm{as}$, and pas, respectively, where arcsecond is an alternative name for second of plane angle.

(e) The unit hectare, and its symbol ha, were adopted by the CIPM in 1879 (PV, 1879, 41). The hectare is used to express land area.

(f) The litre, and the symbol lower-case 1, were adopted by the CIPM in 1879 (PV, 1879, 41). The alternative symbol, capital L, was adopted by the 16th CGPM (1979, Resolution 6; CR, 101 and Metrologia, 1980, 16, 56-57) in order to avoid the risk of confusion between the letter $1(\mathrm{el})$ and the numeral 1 (one).

(g) The tonne, and its symbol t, were adopted by the CIPM in 1879 (PV, 1879, 41). In English speaking countries this unit is usually called "metric ton". 
Table 7 contains units whose values in SI units have to be determined experimentally, and thus have an associated uncertainty. Except for the astronomical unit, all other units in Table 7 are related to fundamental physical constants. The first three units, the non-SI units electronvolt, symbol eV, dalton or unified atomic mass unit, symbol $\mathrm{Da}$ or $\mathrm{u}$, respectively, and the astronomical unit, symbol ua, have been accepted for use with the SI by the CIPM. The units in Table 7 play important roles in a number of specialized fields in which the results of measurements or calculations are most conveniently and usefully expressed in these units. For the electronvolt and the dalton the values depend on the elementary charge $e$ and the Avogadro constant $N_{\mathrm{A}}$, respectively.

There are many other units of this kind, because there are many fields in which it is most convenient to express the results of experimental observations or of theoretical calculations in terms of fundamental constants of nature. The two most important of such unit systems based on fundamental constants are the natural unit (n.u.) system used in high energy or particle physics, and the atomic unit (a.u.) system used in atomic physics and quantum chemistry. In the n.u. system, the base quantities for mechanics are speed, action, and mass, for which the base units are the speed of light in vacuum $c_{0}$, the Planck constant $h$ divided by $2 \pi$, called the reduced Planck constant with symbol $\hbar$, and the mass of the electron $m_{\mathrm{e}}$, respectively. In general these units are not given any special names or symbols but are simply called the n.u. of speed, symbol $c_{0}$, the n.u. of action, symbol $\hbar$, and the n.u. of mass, symbol $m_{\mathrm{e}}$. In this system, time is a derived quantity and the n.u. of time is a derived unit equal to the combination of base units $\hbar / m_{\mathrm{e}} c_{0}^{2}$. Similarly, in the a.u. system, any four of the five quantities charge, mass, action, length, and energy are taken as base quantities. The corresponding base units are the elementary charge $e$, electron mass $m_{\mathrm{e}}$, action $\hbar$, Bohr radius (or bohr) $a_{0}$, and Hartree energy (or hartree) $E_{\mathrm{h}}$, respectively. In this system, time is again a derived quantity and the a.u. of time a derived unit, equal to the combination of units $\hbar / E_{\mathrm{h}}$. Note that $a_{0}=\alpha /\left(4 \pi R_{\infty}\right)$, where $\alpha$ is the fine-structure constant and $R_{\infty}$ is the Rydberg constant; and $E_{\mathrm{h}}=e^{2} /\left(4 \pi \varepsilon_{0} a_{0}\right)=2 R_{\infty} h c_{0}=\alpha^{2} m_{\mathrm{e}} c_{0}^{2}$, where $\varepsilon_{0}$ is the electric constant and has an exact value in the SI.

For information, these ten natural and atomic units and their values in SI units are also listed in Table 7. Because the quantity systems on which these units are based differ so fundamentally from that on which the SI is based, they are not generally used with the SI, and the CIPM has not formally accepted them for use with the International System. To ensure understanding, the final result of a measurement or calculation expressed in natural or atomic units should also always be expressed in the corresponding SI unit. Natural units (n.u.) and atomic units (a.u.) are used only in their own special fields of particle and atomic physics, and quantum chemistry, respectively. Standard uncertainties in the least significant digits are shown in parenthesis after each numerical value. 
Table 7. Non-SI units whose values in SI units must be obtained experimentally

\begin{tabular}{|c|c|c|c|}
\hline Quantity & Name of unit & Symbol for unit & Value in SI units ${ }^{(a)}$ \\
\hline \multicolumn{4}{|c|}{ Units accepted for use with the SI } \\
\hline energy & electronvolt $^{(b)}$ & $\mathrm{eV}$ & $1 \mathrm{eV}=1.60217653(14) \times 10^{-19} \mathrm{~J}$ \\
\hline mass & dalton, ${ }^{(c)}$ & $\mathrm{Da}$ & $1 \mathrm{Da}=1.66053886(28) \times 10^{-27} \mathrm{~kg}$ \\
\hline & unified atomic mass unit & $\mathrm{u}$ & $1 \mathrm{u}=1 \mathrm{Da}$ \\
\hline length & astronomical unit $^{(d)}$ & ua & $1 \mathrm{ua}=1.49597870691(6) \times 10^{11} \mathrm{~m}$ \\
\hline \multicolumn{4}{|c|}{ Natural units (n.u.) } \\
\hline speed & $\begin{array}{l}\text { n.u. of speed } \\
\text { (speed of light in vacuum) }\end{array}$ & $c_{0}$ & $299792458 \mathrm{~m} / \mathrm{s}$ (exact) \\
\hline action & $\begin{array}{l}\text { n.u. of action } \\
\text { (reduced Planck constant) }\end{array}$ & $\hbar$ & $1.05457168(18) \times 10^{-34} \mathrm{~J} \mathrm{~s}$ \\
\hline mass & $\begin{array}{l}\text { n.u. of mass } \\
\text { (electron mass) }\end{array}$ & $m_{\mathrm{e}}$ & $9.1093826(16) \times 10^{-31} \mathrm{~kg}$ \\
\hline time & n.u. of time & $\hbar /\left(m_{\mathrm{e}} c_{0}^{2}\right)$ & $1.2880886677(86) \times 10^{-21} \mathrm{~s}$ \\
\hline \multicolumn{4}{|c|}{ Atomic units (a.u.) } \\
\hline charge & $\begin{array}{l}\text { a.u. of charge, } \\
\text { (elementary charge) }\end{array}$ & $e$ & $1.60217653(14) \times 10^{-19} \mathrm{C}$ \\
\hline mass & $\begin{array}{l}\text { a.u. of mass, } \\
\text { (electron mass) }\end{array}$ & $m_{\mathrm{e}}$ & $9.1093826(16) \times 10^{-31} \mathrm{~kg}$ \\
\hline action & $\begin{array}{l}\text { a.u. of action, } \\
\text { (reduced Planck constant) }\end{array}$ & $\hbar$ & $1.05457168(18) \times 10^{-34} \mathrm{~J} \mathrm{~s}$ \\
\hline length & $\begin{array}{l}\text { a.u. of length, bohr } \\
\text { (Bohr radius) }\end{array}$ & $a_{0}$ & $0.5291772108(18) \times 10^{-10} \mathrm{~m}$ \\
\hline energy & $\begin{array}{l}\text { a.u. of energy, hartree } \\
\text { (Hartree energy) }\end{array}$ & $E_{\mathrm{h}}$ & $4.35974417(75) \times 10^{-18} \mathrm{~J}$ \\
\hline time & a.u. of time & $\hbar / E_{\mathrm{h}}$ & $2.418884326505(16) \times 10^{-17} \mathrm{~s}$ \\
\hline
\end{tabular}

(a) The values in SI units of all units in this table, except the astronomical unit, are taken from the 2002 CODATA set of recommended values of the fundamental physical constants, P.J. Mohr and B.N. Taylor, Rev. Mod. Phys., 2005, 77, 1-107. The standard uncertainty in the last two digits is given in parenthesis (see 5.3.5, p. 133).

(b) The electronvolt is the kinetic energy acquired by an electron in passing through a potential difference of one volt in vacuum. The electronvolt is often combined with the SI prefixes.

(c) The dalton ( $\mathrm{Da})$ and the unified atomic mass unit (u) are alternative names (and symbols) for the same unit, equal to $1 / 12$ times the mass of a free carbon 12 atom, at rest and in its ground state. The dalton is often combined with SI prefixes, for example to express the masses of large molecules in kilodaltons, $\mathrm{kDa}$, or megadaltons, $\mathrm{MDa}$, or to express the values of small mass differences of atoms or molecules in nanodaltons, $\mathrm{nDa}$, or even picodaltons, $\mathrm{pDa}$.

(d) The astronomical unit is approximately equal to the mean Earth-Sun distance. It is the radius of an unperturbed circular Newtonian orbit about the Sun of a particle having infinitesimal mass, moving with a mean motion of 0.01720209895 radians per day (known as the Gaussian constant). The value given for the astronomical unit is quoted from the IERS Conventions 2003 (D.D. McCarthy and G. Petit eds., IERS Technical Note 32, Frankfurt am Main: Verlag des Bundesamts für Kartographie und Geodäsie, 2004, 12). The value of the astronomical unit in metres comes from the JPL ephemerides DE403 (Standish E.M., Report of the IAU WGAS Sub-Group on Numerical Standards, Highlights of Astronomy, Appenzeller ed., Dordrecht: Kluwer Academic Publishers, 1995, 180-184). 
Tables 8 and 9 contain non-SI units that are used by special interest groups for a variety of different reasons. Although the use of SI units is to be preferred for reasons already emphasized, authors who see a particular advantage in using these non-SI units should have the freedom to use the units that they consider to be best suited to their purpose. Since, however, SI units are the international meeting ground in terms of which all other units are defined, those who use units from Tables 8 and 9 should always give the definition of the units they use in terms of SI units.

Table 8 also gives the units of logarithmic ratio quantities, the neper, bel, and decibel. These are dimensionless units that are somewhat different in their nature from other dimensionless units, and some scientists consider that they should not even be called units. They are used to convey information on the nature of the logarithmic ratio quantity concerned. The neper, $\mathrm{Np}$, is used to express the values of quantities whose numerical values are based on the use of the neperian (or natural) logarithm, $\ln =\log _{\mathrm{e}}$. The bel and the decibel, $\mathrm{B}$ and $\mathrm{dB}$, where $1 \mathrm{~dB}=(1 / 10) \mathrm{B}$, are used to express the values of logarithmic ratio quantities whose numerical values are based on the decadic $\operatorname{logarithm}, \lg =\log _{10}$. The way in which these units are interpreted is described in footnotes $(g)$ and $(h)$ of Table 8 . The numerical values of these units are rarely required. The units neper, bel, and decibel have been accepted by the CIPM for use with the International System, but are not considered as SI units.

The SI prefixes are used with two of the units in Table 8, namely, with the bar (e.g. millibar, mbar), and with the bel, specifically for the decibel, $\mathrm{dB}$. The decibel is listed explicitly in the table because the bel is rarely used without the prefix.

Table 8. Other non-SI units

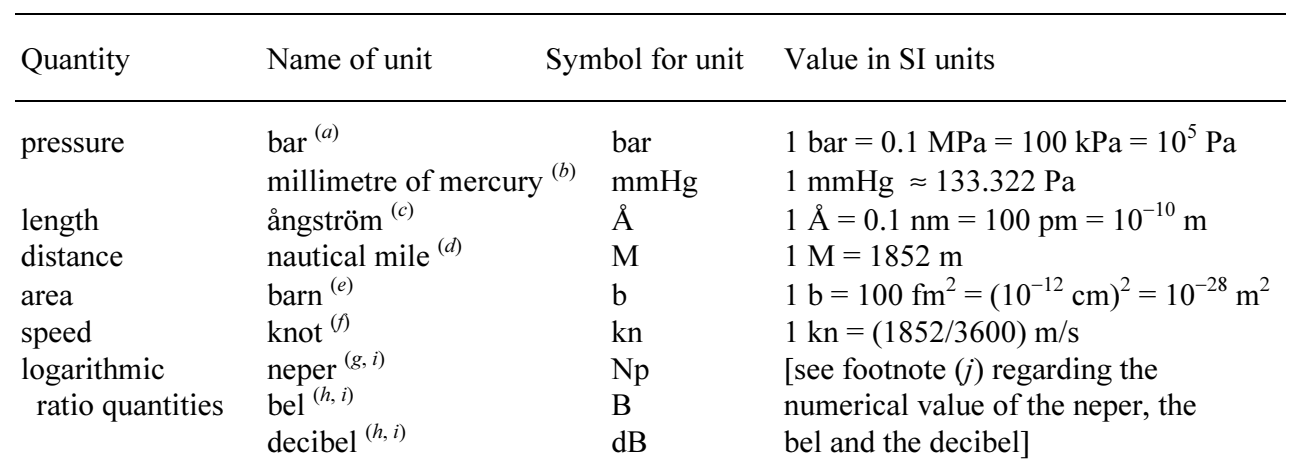

(a) The bar and its symbol are included in Resolution 7 of the 9th CGPM (1948; CR, 70). Since 1982 one bar has been used as the standard pressure for tabulating all thermodynamic data. Prior to 1982 the standard pressure used to be the standard atmosphere, equal to 1.01325 bar, or $101325 \mathrm{~Pa}$.

(b) The millimetre of mercury is a legal unit for the measurement of blood pressure in some countries.

(c) The ångström is widely used by x-ray crystallographers and structural chemists because all chemical bonds lie in the range 1 to 3 ångströms. However it has no official sanction from the CIPM or the CGPM.

(d) The nautical mile is a special unit employed for marine and aerial navigation to express distance. The conventional value given here was adopted by the First International Extraordinary Hydrographic Conference, Monaco 1929, under the name "International nautical mile". As yet there is no internationally agreed symbol, but the symbols $\mathrm{M}, \mathrm{NM}, \mathrm{Nm}$, and nmi are all used; in the table the symbol $\mathrm{M}$ is used. The unit was originally chosen, and continues to be used, because one nautical mile on the surface of the Earth subtends approximately one minute of angle at the centre of the Earth, which is convenient when latitude and longitude are measured in degrees and minutes of angle. 
(e) The barn is a unit of area employed to express cross sections in nuclear physics.

(f) The knot is defined as one nautical mile per hour. There is no internationally agreed symbol, but the symbol $\mathrm{kn}$ is commonly used.

(g) The statement $L_{A}=n \mathrm{~Np}$ (where $n$ is a number) is interpreted to mean that $\ln \left(A_{2} / A_{1}\right)=n$. Thus when $L_{A}=1 \mathrm{~Np}, A_{2} / A_{1}=\mathrm{e}$. The symbol $A$ is used here to denote the amplitude of a sinusoidal signal, and $L_{A}$ is then called the neperian logarithmic amplitude ratio, or the neperian amplitude level difference.

(h) The statement $L_{X}=m \mathrm{~dB}=(m / 10) \mathrm{B}$ (where $m$ is a number) is interpreted to mean that $\lg \left(X / X_{0}\right)$ $=m / 10$. Thus when $L_{X}=1 \mathrm{~B}, X / X_{0}=10$, and when $L_{X}=1 \mathrm{~dB}, X / X_{0}=10^{1 / 10}$. If $X$ denotes a mean square signal or power-like quantity, $L_{X}$ is called a power level referred to $X_{0}$.

(i) In using these units it is important that the nature of the quantity be specified, and that any reference value used be specified. These units are not SI units, but they have been accepted by the CIPM for use with the SI.

(j) The numerical values of the neper, bel, and decibel (and hence the relation of the bel and the decibel to the neper) are rarely required. They depend on the way in which the logarithmic quantities are defined.

Table 9 differs from Table 8 only in that the units in Table 9 are related to the older CGS (centimetre-gram-second) system of units, including the CGS electrical units. In the field of mechanics, the CGS system of units was built upon three quantities and their corresponding base units: the centimetre, the gram, and the second. The CGS electrical units were still derived from only these same three base units, using defining equations different from those used for the SI. Because this can be done in different ways, it led to the establishment of several different systems, namely the CGS-ESU (electrostatic), the CGS-EMU (electromagnetic), and the CGS-Gaussian unit systems. It has always been recognized that the CGS-Gaussian system, in particular, has advantages in certain areas of physics, particularly in classical and relativistic electrodynamics (9th CGPM, 1948, Resolution 6). Table 9 gives the relations between these CGS units and the SI, and lists those CGS units that were assigned special names. As for the units in Table 8, the SI prefixes are used with several of these units (e.g. millidyne, mdyn; milligauss, $\mathrm{mG}$, etc.).

Table 9. Non-SI units associated with the CGS and the CGS-Gaussian system of units

\begin{tabular}{|c|c|c|c|}
\hline Quantity & Name of unit & Symbol for unit & Value in SI units \\
\hline energy & $\operatorname{erg}^{(a)}$ & erg & $1 \mathrm{erg}=10^{-7} \mathrm{~J}$ \\
\hline force & dyne $^{(a)}$ & dyn & $1 \mathrm{dyn}=10^{-5} \mathrm{~N}$ \\
\hline dynamic viscosity & poise $^{(a)}$ & $\mathrm{P}$ & $1 \mathrm{P}=1 \mathrm{dyn} \mathrm{s} \mathrm{cm}-2=0.1 \mathrm{~Pa} \mathrm{~s}$ \\
\hline kinematic viscosity & stokes & St & $1 \mathrm{St}=1 \mathrm{~cm}^{2} \mathrm{~s}^{-1}=10^{-4} \mathrm{~m}^{2} \mathrm{~s}^{-1}$ \\
\hline luminance & stilb $^{(a)}$ & $\mathrm{sb}$ & $1 \mathrm{sb}=1 \mathrm{~cd} \mathrm{~cm}^{-2}=10^{4} \mathrm{~cd} \mathrm{~m}^{-2}$ \\
\hline illuminance & phot & $\mathrm{ph}$ & $1 \mathrm{ph}=1 \mathrm{~cd} \mathrm{sr} \mathrm{cm}^{-2}=10^{4} \mathrm{~lx}$ \\
\hline acceleration & gal $^{(b)}$ & Gal & $1 \mathrm{Gal}=1 \mathrm{~cm} \mathrm{~s}^{-2}=10^{-2} \mathrm{~m} \mathrm{~s}^{-2}$ \\
\hline magnetic flux & maxwell ${ }^{(c)}$ & $\mathrm{Mx}$ & $1 \mathrm{Mx}=1 \mathrm{G} \mathrm{cm}^{2}=10^{-8} \mathrm{~Wb}$ \\
\hline magnetic flux density & gauss $^{(c)}$ & G & $1 \mathrm{G}=1 \mathrm{Mx} \mathrm{cm}^{-2}=10^{-4} \mathrm{~T}$ \\
\hline magnetic field & œrsted $^{(c)}$ & Oe & $1 \mathrm{Oe} \hat{=}\left(10^{3} / 4 \pi\right) \mathrm{A} \mathrm{m}^{-1}$ \\
\hline
\end{tabular}

(a) This unit and its symbol were included in Resolution 7 of the 9th CGPM (1948; CR, 70).

(b) The gal is a special unit of acceleration employed in geodesy and geophysics to express acceleration due to gravity. 
(c) These units are part of the so-called "electromagnetic" three-dimensional CGS system based on unrationalized quantity equations, and must be compared with care to the corresponding unit of the International System which is based on rationalized equations involving four dimensions and four quantities for electromagnetic theory. The magnetic flux, $\Phi$, and the magnetic flux density, $B$, are defined by similar equations in the CGS system and the SI, so that the corresponding units can be related as in the table. However, the unrationalized magnetic field, $H($ unrationalized $)=4 \pi \times H$ (rationalized $).$ The equivalence symbol $\hat{=}$ is used to indicate that when $H($ unrationalized $)=1$ Oe, $H($ rationalized $)=\left(10^{3} / 4 \pi\right) \mathrm{A} \mathrm{m}^{-1}$.

\subsection{Other non-SI units not recommended for use}

There are many more non-SI units, which are too numerous to list here, which are either of historical interest, or are still used but only in specialized fields (for example, the barrel of oil) or in particular countries (the inch, foot, and yard). The CIPM can see no case for continuing to use these units in modern scientific and technical work. However, it is clearly a matter of importance to be able to recall the relation of these units to the corresponding SI units, and this will continue to be true for many years. The CIPM has therefore decided to compile a list of the conversion factors to the SI for such units and to make this available on the BIPM website at www.bipm.org/en/si/si brochure/chapter4/conversion_factors.html. 


\section{$5 \quad$ Writing unit symbols and names, and expressing the values of quantities}

General principles for the writing of unit symbols and numbers were first given by the 9th CGPM (1948, Resolution 7). These were subsequently elaborated by ISO, IEC, and other international bodies. As a consequence, there now exists a general consensus on how unit symbols and names, including prefix symbols and names, as well as quantity symbols should be written and used, and how the values of quantities should be expressed. Compliance with these rules and style conventions, the most important of which are presented in this chapter, supports the readability of scientific and technical papers.

\subsection{Unit symbols}

Unit symbols are printed in roman (upright) type regardless of the type used in the surrounding text. They are printed in lower-case letters unless they are derived from a proper name, in which case the first letter is a capital letter.

$\mathrm{m}$, metre $\mathrm{s}$, second

$\mathrm{Pa}$, pascal

An exception, adopted by the 16th CGPM (1979, Resolution 6), is that either capital $\mathrm{L}$ or lower-case 1 is allowed for the litre, in order to avoid possible confusion between the numeral 1 (one) and the lower-case letter 1 (el).

A multiple or sub-multiple prefix, if used, is part of the unit and precedes the unit symbol without a separator. A prefix is never used in isolation, and compound prefixes are never used.

Unit symbols are mathematical entities and not abbreviations. Therefore, they are not followed by a period except at the end of a sentence, and one must neither use the plural nor mix unit symbols and unit names within one expression, since names are not mathematical entities.

In forming products and quotients of unit symbols the normal rules of algebraic multiplication or division apply. Multiplication must be indicated by a space or a half-high (centred) dot (.), since otherwise some prefixes could be misinterpreted as a unit symbol. Division is indicated by a horizontal line, by a solidus (oblique stroke, /) or by negative exponents. When several unit symbols are combined, care should be taken to avoid ambiguities, for example by using brackets or negative exponents. A solidus must not be used more than once in a given expression without brackets to remove ambiguities.

It is not permissible to use abbreviations for unit symbols or unit names, such as sec (for either $\mathrm{s}$ or second), sq. $\mathrm{mm}$ (for either $\mathrm{mm}^{2}$ or square millimetre), $\mathrm{cc}$ (for either $\mathrm{cm}^{3}$ or cubic centimetre), or mps (for either $\mathrm{m} / \mathrm{s}$ or metre per second). The use of the correct symbols for SI units, and for units in general, as listed in earlier chapters of this Brochure, is mandatory. In this way ambiguities and misunderstandings in the values of quantities are avoided.

$$
\begin{aligned}
& \mathrm{N} \\
& \text { for } \\
& \mathrm{m} / \mathrm{s} \\
& \text { for } \\
& \mathrm{ms} \\
& \mathrm{m}
\end{aligned}
$$




\subsection{Unit names}

Unit names are normally printed in roman (upright) type, and they are treated like ordinary nouns. In English, the names of units start with a lower-case letter (even when the symbol for the unit begins with a capital letter), except at the beginning of a sentence or in capitalized material such as a title. In keeping with this rule, the correct spelling of the name of the unit with the symbol ${ }^{\circ} \mathrm{C}$ is "degree Celsius" (the unit degree begins with a lower-case $d$ and the modifier Celsius begins with an upper-case $\mathrm{C}$ because it is a proper name).

Although the values of quantities are normally expressed using symbols for numbers and symbols for units, if for some reason the unit name is more appropriate than the unit symbol, the unit name should be spelled out in full.

When the name of a unit is combined with the name of a multiple or sub-multiple prefix, no space or hyphen is used between the prefix name and the unit name. The combination of prefix name plus unit name is a single word. See also Chapter 3 , Section 3.1 .

In both English and in French, however, when the name of a derived unit is formed from the names of individual units by multiplication, then either a space or a hyphen is used to separate the names of the individual units.

In both English and in French modifiers such as "squared" or "cubed" are used in the names of units raised to powers, and they are placed after the unit name. However, in the case of area or volume, as an alternative the modifiers "square" or "cubic" may be used, and these modifiers are placed before the unit name, but this applies only in English.

\subsection{Rules and style conventions for expressing values of quantities}

\subsubsection{Value and numerical value of a quantity, and the use of quantity calculus}

The value of a quantity is expressed as the product of a number and a unit, and the number multiplying the unit is the numerical value of the quantity expressed in that unit. The numerical value of a quantity depends on the choice of unit. Thus the value of a particular quantity is independent of the choice of unit, although the numerical value will be different for different units.

Symbols for quantities are generally single letters set in an italic font, although they may be qualified by further information in subscripts or superscripts or in brackets. Thus $C$ is the recommended symbol for heat capacity, $C_{\mathrm{m}}$ for molar heat capacity, $C_{\mathrm{m}, p}$ for molar heat capacity at constant pressure, and $C_{\mathrm{m}, V}$ for molar heat capacity at constant volume.

Recommended names and symbols for quantities are listed in many standard references, such as the ISO Standard 31 Quantities and Units, the IUPAP SUNAMCO Red Book Symbols, Units and Nomenclature in Physics, and the IUPAC Green Book Quantities, Units and Symbols in Physical Chemistry. However, symbols for quantities are recommendations (in contrast to symbols for units, for which the use of the correct form is mandatory). In particular circumstances authors may wish to use a symbol of their own choice for a quantity, for example in order to avoid a conflict arising from the use of the same symbol for two different quantities. In such

The same value of a speed $v=\mathrm{d} x / \mathrm{d} t$ of a particle might be given by either of the expressions $v=25 \mathrm{~m} / \mathrm{s}=90 \mathrm{~km} / \mathrm{h}$, where 25 is the numerical value of the speed in the unit metres per second, and 90 is the numerical value of the speed in the unit kilometres per hour. 
cases, the meaning of the symbol must be clearly stated. However, neither the name of a quantity, nor the symbol used to denote it, should imply any particular choice of unit.

Symbols for units are treated as mathematical entities. In expressing the value of a quantity as the product of a numerical value and a unit, both the numerical value and the unit may be treated by the ordinary rules of algebra. This procedure is described as the use of quantity calculus, or the algebra of quantities. For example, the equation $T=293 \mathrm{~K}$ may equally be written $T / \mathrm{K}=293$. It is often convenient to write the quotient of a quantity and a unit in this way for the heading of a column in a table, so that the entries in the table are all simply numbers. For example, a table of vapour pressure against temperature, and the natural logarithm of vapour pressure against reciprocal temperature, may be formatted as shown below.

\begin{tabular}{llll}
\hline$T / \mathrm{K}$ & $10^{3} \mathrm{~K} / T$ & $p / \mathrm{MPa}$ & $\ln (p / \mathrm{MPa})$ \\
\hline 216.55 & 4.6179 & 0.5180 & -0.6578 \\
273.15 & 3.6610 & 3.4853 & 1.2486 \\
304.19 & 3.2874 & 7.3815 & 1.9990 \\
\hline
\end{tabular}

The axes of a graph may also be labelled in this way, so that the tick marks are labelled only with numbers, as in the graph below.

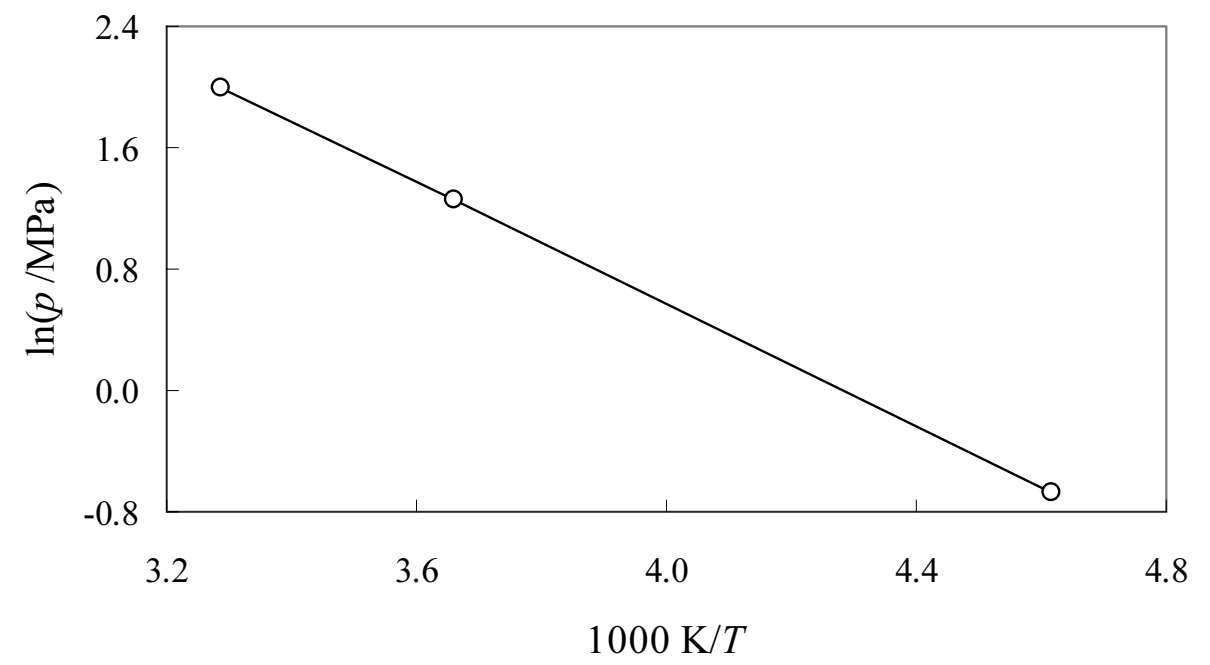

Algebraically equivalent forms may be used in place of $10^{3} \mathrm{~K} / T$, such as $\mathrm{kK} / T$, or $10^{3}(T / \mathrm{K})^{-1}$.

\subsubsection{Quantity symbols and unit symbols}

Just as the quantity symbol should not imply any particular choice of unit, the unit symbol should not be used to provide specific information about the quantity, and should never be the sole source of information on the quantity. Units are never qualified by further information about the nature of the quantity; any extra information on the nature of the quantity should be attached to the quantity symbol and not to the unit symbol.

For example:

The maximum electric potential difference is $U_{\max }=1000 \mathrm{~V}$

but not $U=1000 \mathrm{~V}_{\max }$. The mass fraction of copper in the sample of silicon is $w(\mathrm{Cu})=1.3 \times 10^{-6}$ but not $1.3 \times 10^{-6} \mathrm{w} / \mathrm{w}$. 


\title{
5.3.3 Formatting the value of a quantity
}

The numerical value always precedes the unit, and a space is always used to separate the unit from the number. Thus the value of the quantity is the product of the number and the unit, the space being regarded as a multiplication sign (just as a space between units implies multiplication). The only exceptions to this rule are for the unit symbols for degree, minute, and second for plane angle, ${ }^{\circ},{ }^{\prime}$, and ", respectively, for which no space is left between the numerical value and the unit symbol.

This rule means that the symbol ${ }^{\circ} \mathrm{C}$ for the degree Celsius is preceded by a space when one expresses values of Celsius temperature $t$.

Even when the value of a quantity is used as an adjective, a space is left between the numerical value and the unit symbol. Only when the name of the unit is spelled out would the ordinary rules of grammar apply, so that in English a hyphen would be used to separate the number from the unit.

In any one expression, only one unit is used. An exception to this rule is in expressing the values of time and of plane angles using non-SI units. However, for plane angles it is generally preferable to divide the degree decimally. Thus one would write $22.20^{\circ}$ rather than $22^{\circ} 12^{\prime}$, except in fields such as navigation, cartography, astronomy, and in the measurement of very small angles.

\subsubsection{Formatting numbers, and the decimal marker}

The symbol used to separate the integral part of a number from its decimal part is called the decimal marker. Following the 22nd CGPM (2003, Resolution 10), the decimal marker "shall be either the point on the line or the comma on the line." The decimal marker chosen should be that which is customary in the context concerned.

If the number is between +1 and -1 , then the decimal marker is always preceded by a zero.

\author{
$l=10.234 \mathrm{~m}$ \\ but not \\ $l=10 \mathrm{~m} 23.4 \mathrm{~cm}$
}
$t=30.2{ }^{\circ} \mathrm{C}$,
but not $t=30.2^{\circ} \mathrm{C}$,

a $10 \mathrm{k} \Omega$ resistor

a 35-millimetre film

Following the 9th CGPM (1948, Resolution 7) and the 22nd CGPM (2003, Resolution 10), for numbers with many digits the digits may be divided into groups of three by a thin space, in order to facilitate reading. Neither dots nor commas are inserted in the spaces between groups of three. However, when there are only four digits before or after the decimal marker, it is customary not to use a space to isolate a single digit. The practice of grouping digits in this way is a matter of choice; it is not

$-0.234$

but not -.234

43279.16829

but not $43,279.168,29$

either 3279.1683

or $\quad 3279.1683$ always followed in certain specialized applications such as engineering drawings, financial statements, and scripts to be read by a computer.

For numbers in a table, the format used should not vary within one column.

\subsubsection{Expressing the measurement uncertainty in the value of a quantity}

The uncertainty that is associated with the estimated value of a quantity should be evaluated and expressed in accordance with the Guide to the Expression of Uncertainty in Measurement [ISO, 1995]. The standard uncertainty (i.e. estimated standard deviation, coverage factor $k=1$ ) associated with a quantity $x$ is denoted by $u(x)$. A convenient way to represent the uncertainty is given in the following example:

$$
m_{\mathrm{n}}=1.67492728(29) \times 10^{-27} \mathrm{~kg} \text {. }
$$

where $m_{\mathrm{n}}$ is the symbol for the quantity (in this case the mass of a neutron), and the number in parenthesis is the numerical value of the combined standard uncertainty of the estimated value of $m_{\mathrm{n}}$ referred to the last two digits of the quoted value; in this 
case $u\left(m_{\mathrm{n}}\right)=0.00000029 \times 10^{-27} \mathrm{~kg}$. If any coverage factor, $k$, different from one, is used, this factor must be stated.

\subsubsection{Multiplying or dividing quantity symbols, the values of quantities, or numbers}

When multiplying or dividing quantity symbols any of the following methods may be used: $a b, a b, a \cdot b, a \times b, a / b, \frac{a}{b}, a b^{-1}$.

When multiplying the value of quantities either a multiplication sign, $\times$, or brackets should be used, not a half-high (centred) dot. When multiplying numbers only the multiplication sign, $\times$, should be used.

When dividing the values of quantities using a solidus, brackets are used to remove ambiguities.

Examples:

$F=m a$ for force equals mass times acceleration

$(53 \mathrm{~m} / \mathrm{s}) \times 10.2 \mathrm{~s}$ or $(53 \mathrm{~m} / \mathrm{s})(10.2 \mathrm{~s})$

$25 \times 60.5$

but not $25 \cdot 60.5$

$(20 \mathrm{~m}) /(5 \mathrm{~s})=4 \mathrm{~m} / \mathrm{s}$

$(a / b) / c$, not $a / b / c$

\subsubsection{Stating values of dimensionless quantities, or quantities of dimension one}

As discussed in Section 2.2.3, the coherent SI unit for dimensionless quantities, also termed quantities of dimension one, is the number one, symbol 1. Values of such quantities are expressed simply as numbers. The unit symbol 1 or unit name "one" are not explicitly shown, nor are special symbols or names given to the unit one, apart from a few exceptions as follows. For the quantity plane angle, the unit one is given the special name radian, symbol rad, and for the quantity solid angle, the unit one is given the special name steradian, symbol sr. For the logarithmic ratio quantities, the special names neper, symbol $\mathrm{Np}$, bel, symbol $\mathrm{B}$, and decibel, symbol $\mathrm{dB}$, are used (see 4.1 and Table 8, p. 127).

Because SI prefix symbols can neither be attached to the symbol 1 nor to the name "one", powers of 10 are used to express the values of particularly large or small dimensionless quantities.

In mathematical expressions, the internationally recognized symbol \% (percent) may be used with the SI to represent the number 0.01 . Thus, it can be used to express the values of dimensionless quantities. When it is used, a space separates the number and the symbol \%. In expressing the values of dimensionless quantities in this way, the symbol \% should be used rather than the name "percent".

In written text, however, the symbol \% generally takes the meaning of "parts per hundred".

Phrases such as "percentage by mass", "percentage by volume", or "percentage by amount of substance" should not be used; the extra information on the quantity should instead be conveyed in the name and symbol for the quantity.

In expressing the values of dimensionless fractions (e.g. mass fraction, volume fraction, relative uncertainties), the use of a ratio of two units of the same kind is sometimes useful.

The term "ppm", meaning $10^{-6}$ relative value, or 1 in $10^{6}$, or parts per million, is also used. This is analogous to the meaning of percent as parts per hundred. The terms "parts per billion", and "parts per trillion", and their respective abbreviations "ppb", and "ppt", are also used, but their meanings are language dependent. For this reason the terms ppb and ppt are best avoided. (In English-speaking countries, a billion is now generally taken to be $10^{9}$ and a trillion to be $10^{12}$; however, a billion may still

$n=1.51$,

but not $n=1.51 \times 1$, where $n$ is the quantity symbol for refractive index.

$x_{\mathrm{B}}=0.0025=0.25 \%$, where $x_{\mathrm{B}}$ is the quantity symbol for amount fraction (mole fraction) of entity B.

The mirror reflects $95 \%$ of the incident photons.

$\varphi=3.6 \%$,

but not $\varphi=3.6 \%(V / V)$, where $\varphi$ denotes volume fraction.

$x_{\mathrm{B}}=2.5 \times 10^{-3}$

$=2.5 \mathrm{mmol} / \mathrm{mol}$

$u_{\mathrm{r}}(U)=0.3 \mu \mathrm{V} / \mathrm{V}$, where $u_{\mathrm{r}}(U)$ is the relative uncertainty of the measured voltage $U$. 
sometimes be interpreted as $10^{12}$ and a trillion as $10^{18}$. The abbreviation ppt is also sometimes read as parts per thousand, adding further confusion.)

When any of the terms \%, ppm, etc., are used it is important to state the dimensionless quantity whose value is being specified. 



\section{Appendix 1. Decisions of the CGPM and the CIPM}

This appendix lists those decisions of the CGPM and the CIPM that bear directly upon definitions of the units of the SI, prefixes defined for use as part of the SI, and conventions for the writing of unit symbols and numbers. It is not a complete list of CGPM and CIPM decisions. For a complete list, reference must be made to successive volumes of the Comptes Rendus des Séances de la Conférence Générale des Poids et Mesures (CR) and Procès-Verbaux des Séances du Comité International des Poids et Mesures (PV) or, for recent decisions, to Metrologia.

Since the SI is not a static convention, but evolves following developments in the science of measurement, some decisions have been abrogated or modified; others have been clarified by additions. Decisions that have been subject to such changes are identified by an asterisk $\left(^{*}\right)$ and are linked by a note to the modifying decision.

The original text of each decision (or its translation) is shown in a different font (sans serif) of normal weight to distinguish it from the main text. The asterisks and notes were added by the BIPM to make the text more understandable. They do not form part of the original text.

The decisions of the CGPM and CIPM are listed in this appendix in strict chronological order, from 1889 to 2005 , in order to preserve the continuity with which they were taken. However in order to make it easy to locate decisions related to particular topics a table of contents is included below, ordered by subject, with page references to the particular meetings at which decisions relating to each subject were taken. 


\section{Table of Contents of Appendix 1}

Decisions relating to the establishment of the SI page

9th CGPM, 1948: decision to establish the SI 145

10th CGPM, 1954: decision on the first six base units 147

CIPM 1956: $\quad$ decision to adopt the name "Système International d'Unités"

11th CGPM, 1960: $\quad$ confirms the name and the abbreviation "SI", 149

names prefixes from tera to pico, $\quad 149$

establishes the supplementary units rad and sr, $\quad 149$

lists some derived units $\quad 150$

CIPM, 1969: $\quad$ declarations concerning base, supplementary, derived and coherent units, and the use of prefixes 155

CIPM, $2001 \quad$ "SI units" and "units of the SI" 166

Decisions relating to the base units of the SI

Length

1st CGPM, 1889: $\quad$ sanction of the prototype metre 142

7th CGPM, 1927: definition and use of the prototype metre 143

11th CGPM, 1960: $\quad$ redefinition of the metre in terms of krypton 86 radiation $\quad 148$

15th CGPM, 1975: $\quad$ recommends value for the speed of light 157

17th CGPM, 1983: $\quad$ redefinition of the metre using the speed of light, 160 $\begin{array}{ll}\text { realization of the definition of the metre } & 161\end{array}$

CIPM, 2002: $\quad$ specifies the rules for the practical realization of the definition of the metre 166

CIPM, 2003: $\quad$ revision of the list of recommended radiations $\quad 169$

CIPM, 2005: $\quad$ revision of the list of recommended radiations $\quad 171$

\section{Mass}

1st CGPM, 1889: $\quad$ sanction of the prototype kilogram 142

3rd CGPM, 1901: declaration on distinguishing mass and weight, and on the conventional value of $g_{n} \quad 143$

CIPM, 1967: $\quad$ declaration on applying prefixes to the gram

21st CGPM, 1999: $\quad$ future redefinition of the kilogram 165

\section{Time}

CIPM, 1956: $\quad$ definition of the second as a fraction of the tropical year $1900 \quad 147$

11th CGPM, 1960: $\quad$ ratifies the CIPM 1956 definition of the second 148 
CIPM, 1964: declares the caesium 133 hyperfine transition

\section{2th CGPM, 1964: to be the recommended standard}

empowers CIPM to investigate atomic

13th CGPM, 1967/68: defines the second in terms of the caesi

14th CGPM, 1971: requests the CIPM to define and establish

15th CGPM, 1975: endorses the use of Coordinated Universal Time, UTC

\section{Electrical units}

CIPM, 1946:

definitions of mechanical and electrical units in the SI

14th CGPM, 1971: adopts the name siemens, symbol S, for electrical conductance

18th CGPM, 1987: forthcoming adjustment to the representations of the volt and of the ohm

CIPM, 1988:

Josephson effect

CIPM, 1988:

quantum Hall effect

CIPM, 2000:

realization of the ohm using the value of the

von Klitzing constant

\section{Thermodynamic temperature}

9th CGPM, 1948: $\quad$ adopts the triple point of water as the thermodynamic reference point,

adopts the zero of Celsius temperature to be

0.01 degree below the triple point

CIPM, 1948: $\quad$ adopts the name degree Celsius for the Celsius temperature scale

10th CGPM, 1954: defines thermodynamic temperature such that the triple point of water is 273.16 degrees Kelvin exactly,

13th CGPM, 1967/68: decides formal definition of the kelvin, symbol K

\section{Amount of substance}

14th CGPM, 1971: definition of the mole, symbol mol, as a seventh 
CIPM, 1946: definition of photometric units, new candle and new lumen

13th CGPM, 1967/68: defines the candela, symbol cd, in terms of a black body

\section{Decisions relating to SI derived and supplementary units}

\section{SI derived units}

12th CGPM, 1964: accepts the continued use of the curie as a non-SI unit

13th CGPM, 1967/68: lists some examples of derived units

15th CGPM, 1975: $\quad$ adopts the special names becquerel, Bq, and gray, Gy

CIPM, 2002: modifies the relationship between absorbed dose and dose equivalent

\section{Supplementary units}

CIPM, 1980: $\quad$ decides to interpret supplementary units as dimensionless derived units

20th CGPM, 1995: decides to abrogate the class of supplementary units, and confirms the CIPM interpretation that they are dimensionless derived units

Decisions concerning terminology and the acceptance of units for use with the SI

\section{SI prefixes}

12th CGPM, 1964: decides to add femto and atto to the list of prefixes

Unit symbols and numbers

9th CGPM, 1948: decides rules for printing unit symbols

\section{Unit names}

13th CGPM, 1967/68: abrogates the use of the micron and new candle as units accepted for use with the SI

The decimal marker

22nd CGPM, 2003: decides to allow the use of the point or the comma on the line as the decimal marker 
3rd CGPM, 1901: defines the litre as the volume of $1 \mathrm{~kg}$ of water

11th CGPM, 1960: requests the CIPM to report on the difference

between the litre and the cubic decimetre

CIPM, 1961:

recommends that volume be expressed in SI units and not in litres

12th CGPM, 1964: abrogates the former definition of the litre, recommends that litre may be used as a special name for the cubic decimetre

16th CGPM, 1979: decides, as an exception, to allow both 1 and $\mathrm{L}$ as symbols for the litre 
1st CGPM, 1889

I Sanction of the international prototypes of the metre and the kilogram (CR, 34-38)*

The Conférence Générale des Poids et Mesures,

\section{considering}

- the "Compte rendu of the President of the Comite International des Poids et Mesures (CIPM)" and the "Report of the CIPM", which show that, by the collaboration of the French section of the International Metre Commission and of the CIPM, the fundamental measurements of the international and national prototypes of the metre and of the kilogram have been made with all the accuracy and reliability which the present state of science permits;

- that the international and national prototypes of the metre and the kilogram are made of an alloy of platinum with 10 per cent iridium, to within 0.0001 ;

- the equality in length of the international Metre and the equality in mass of the international Kilogram with the length of the Metre and the mass of the Kilogram kept in the Archives of France;

- that the differences between the national Metres and the international Metre lie within 0.01 millimetre and that these differences are based on a hydrogen thermometer scale which can always be reproduced thanks to the stability of hydrogen, provided identical conditions are secured;

- that the differences between the national Kilograms and the international Kilogram lie within 1 milligram;

- that the international Metre and Kilogram and the national Metres and Kilograms fulfil the requirements of the Metre Convention,

\section{sanctions}

A. As regards international prototypes:

1. The Prototype of the metre chosen by the CIPM. This prototype, at the temperature of melting ice, shall henceforth represent the metric unit of length.

2. The Prototype of the kilogram adopted by the CIPM. This prototype shall henceforth be considered as the unit of mass.

3. The hydrogen thermometer centigrade scale in terms of which the equations of the prototype Metres have been established.

B. As regards national prototypes: .....

\section{3rd CGPM, 1901}

I Declaration concerning the definition of the litre (CR, 38-39)*

$\cdots$

The Conference declares

1. The unit of volume, for high accuracy determinations, is the volume occupied by a mass of 1 kilogram of pure water, at its maximum density and at standard atmospheric pressure: this volume is called "litre".

2.
* The definition of the metre was abrogated in 1960 by the 11 th CGPM (Resolution 6, see p. 148).

* This definition was abrogated in 1964 by the 12th CGPM (Resolution 6, see p. 152). 


\section{Declaration on the unit of mass and on the definition of weight; conventional value of $g_{\mathbf{n}}(\mathrm{CR}, 70)$}

Taking into account the decision of the Comité International des Poids et Mesures of 15 October 1887, according to which the kilogram has been defined as unit of mass;

Taking into account the decision contained in the sanction of the prototypes of the Metric System, unanimously accepted by the Conférence Générale des Poids et Mesures on 26 September 1889;

Considering the necessity to put an end to the ambiguity which in current practice still exists on the meaning of the word weight, used sometimes for mass, sometimes for mechanical force;

\section{The Conference declares}

1. The kilogram is the unit of mass; it is equal to the mass of the international prototype of the kilogram;

2. The word "weight" denotes a quantity of the same nature as a "force": the weight of a body is the product of its mass and the acceleration due to gravity; in particular, the standard weight of a body is the product of its mass and the standard acceleration due to gravity;

3. The value adopted in the International Service of Weights and Measures for the standard acceleration due to gravity is $980.665 \mathrm{~cm} / \mathrm{s}^{2}$, value already stated in the laws of some countries.

\section{7th CGPM, 1927}

\section{Definition of the metre by the international Prototype $(\mathrm{CR}, 49)^{*}$}

The unit of length is the metre, defined by the distance, at $0^{\circ}$, between the axes of the two central lines marked on the bar of platinum-iridium kept at the Bureau International des Poids et Mesures and declared Prototype of the metre by the 1st Conférence Générale des Poids et Mesures, this bar being subject to standard atmospheric pressure and supported on two cylinders of at least one centimetre diameter, symmetrically placed in the same horizontal plane at a distance of $571 \mathrm{~mm}$ from each other.

\section{CIPM, 1946}

I Definitions of photometric units (PV, 20, 119-122)*

\section{Resolution}

4. The photometric units may be defined as follows:

New candle (unit of luminous intensity). - The value of the new candle is such that the brightness of the full radiator at the temperature of solidification of platinum is 60 new candles per square centimetre.

New lumen (unit of luminous flux). - The new lumen is the luminous flux emitted in unit solid angle (steradian) by a uniform point source having a luminous intensity of 1 new candle.

5. ...
This value of $g_{\mathrm{n}}$ was the conventional reference for calculating the now obsolete unit kilogram force.

\section{* This definition was} abrogated in 1960 by the 11th CGPM (Resolution 6, see p. 148).

\footnotetext{
* The two definitions contained in this Resolution were ratified in 1948 by the 9th CGPM, which also approved the name candela given to the "new candle" (CR, 54). For the lumen the qualifier "new" was later abandoned. This definition was modified in 1967 by the 13th CGPM (Resolution 5, see p. 154).
} 
I Definitions of electric units (PV, 20, 132-133)

\section{Resolution 2}

4. (A) Definitions of the mechanical units which enter the definitions of electric units:

Unit of force. - The unit of force [in the MKS (metre, kilogram, second) system] is the force which gives to a mass of 1 kilogram an acceleration of 1 metre per second, per second.

Joule (unit of energy or work). - The joule is the work done when the point of application of $1 \mathrm{MKS}$ unit of force [newton] moves a distance of 1 metre in the direction of the force.

Watt (unit of power). - The watt is the power which in one second gives rise to energy of 1 joule.

(B) Definitions of electric units. The Comité International des Poids et Mesures (CIPM) accepts the following propositions which define the theoretical value of the electric units:

Ampere (unit of electric current). - The ampere is that constant current which, if maintained in two straight parallel conductors of infinite length, of negligible circular cross-section, and placed 1 metre apart in vacuum, would produce between these conductors a force equal to $2 \times 10^{-7}$ MKS unit of force [newton] per metre of length.

Volt (unit of potential difference and of electromotive force). - The volt is the potential difference between two points of a conducting wire carrying a constant current of 1 ampere, when the power dissipated between these points is equal to 1 watt.

Ohm (unit of electric resistance). - The ohm is the electric resistance between two points of a conductor when a constant potential difference of 1 volt, applied to these points, produces in the conductor a current of 1 ampere, the conductor not being the seat of any electromotive force.

Coulomb (unit of quantity of electricity). - The coulomb is the quantity of electricity carried in 1 second by a current of 1 ampere.

Farad (unit of capacitance). - The farad is the capacitance of a capacitor between the plates of which there appears a potential difference of 1 volt when it is charged by a quantity of electricity of 1 coulomb.

Henry (unit of electric inductance). - The henry is the inductance of a closed circuit in which an electromotive force of 1 volt is produced when the electric current in the circuit varies uniformly at the rate of 1 ampere per second.

Weber (unit of magnetic flux). - The weber is the magnetic flux which, linking a circuit of one turn, would produce in it an electromotive force of 1 volt if it were reduced to zero at a uniform rate in 1 second.

\section{9th CGPM, 1948}

I Triple point of water; thermodynamic scale with a single fixed point; unit of quantity of heat (joule) (CR, 55 and 63 )

\section{Resolution 3}

1. With present-day techniques, the triple point of water is capable of providing a thermometric reference point with an accuracy higher than can be obtained from the melting point of ice.

In consequence the Comité Consultatif de Thermométrie et Calorimétrie (CCTC) considers that the zero of the centesimal thermodynamic scale must be defined as the temperature 0.0100 degree below that of the triple point of water.
The definitions contained in this Resolution were ratified in 1948 by the 9th CGPM (CR, 49), which also adopted the name newton (Resolution 7) for the MKS unit of force. 
2. The CCTC accepts the principle of an absolute thermodynamic scale with a single fundamental fixed point, at present provided by the triple point of pure water, the absolute temperature of which will be fixed at a later date.

The introduction of this new scale does not affect in any way the use of the International Scale, which remains the recommended practical scale.

3. The unit of quantity of heat is the joule.

Note: It is requested that the results of calorimetric experiments be as far as possible expressed in joules. If the experiments are made by comparison with the rise of temperature of water (and that, for some reason, it is not possible to avoid using the calorie), the information necessary for conversion to joules must be provided. The CIPM, advised by the CCTC, should prepare a table giving, in joules per degree, the most accurate values that can be obtained from experiments on the specific heat of water.

A table, prepared in response to this request, was approved and published by the CIPM in 1950 (PV, 22, 92).

\section{【 Adoption of "degree Celsius" [CIPM, 1948 (PV, 21, 88) and 9th CGPM, $1948(\mathrm{CR}, 64)]$}

From three names (“degree centigrade", "centesimal degree", "degree Celsius") proposed to denote the degree of temperature, the CIPM has chosen "degree Celsius" (PV, 21, 88).

This name is also adopted by the 9th CGPM (CR, 64).

\section{Proposal for establishing a practical system of units of measurement $(\mathrm{CR}, 64)$}

\section{Resolution 6}

The Conférence Générale des Poids et Mesures (CGPM),

\section{considering}

- that the Comité International des Poids et Mesures (CIPM) has been requested by the International Union of Physics to adopt for international use a practical Système International d'Unités; that the International Union of Physics recommends the MKS system and one electric unit of the absolute practical system, but does not recommend that the CGS system be abandoned by physicists;

- that the CGPM has itself received from the French Government a similar request, accompanied by a draft to be used as basis of discussion for the establishment of a complete specification of units of measurement;

instructs the CIPM:

- to seek by an energetic, active, official enquiry the opinion of scientific, technical and educational circles of all countries (offering them, in fact, the French document as basis);

- to gather and study the answers;

- to make recommendations for a single practical system of units of measurement, suitable for adoption by all countries adhering to the Metre Convention. 
Writing and printing of unit symbols and of numbers $(\mathrm{CR}, 70)^{\star}$

\section{Resolution 7}

\section{Principles}

Roman (upright) type, in general lower-case, is used for symbols of units; if, however, the symbols are derived from proper names, capital roman type is used. These symbols are not followed by a full stop.

In numbers, the comma (French practice) or the dot (British practice) is used only to separate the integral part of numbers from the decimal part. Numbers may be divided in groups of three in order to facilitate reading; neither dots nor commas are ever inserted in the spaces between groups.

\begin{tabular}{llll} 
Unit & Symbol & Unit & Symbol \\
\hline - metre & $\mathrm{m}$ & ampere & $\mathrm{A}$ \\
- square metre & $\mathrm{m}^{2}$ & volt & $\mathrm{V}$ \\
- cubic metre & $\mathrm{m}^{3}$ & watt & $\mathrm{W}$ \\
- micron & $\mu$ & ohm & $\Omega$ \\
- litre & $\mathrm{I}$ & coulomb & $\mathrm{C}$ \\
- gram & $\mathrm{g}$ & farad & $\mathrm{F}$ \\
- tonne & $\mathrm{t}$ & henry & $\mathrm{H}$ \\
second & $\mathrm{S}$ & hertz & $\mathrm{Hz}$ \\
erg & $\mathrm{erg}$ & poise & $\mathrm{P}$ \\
dyne & $\mathrm{dyn}$ & newton & $\mathrm{N}$ \\
degree Celsius & ${ }^{\circ} \mathrm{C}$ & $\bullet$ candela (new candle) & $\mathrm{cd}$ \\
- degree absolute & ${ }^{\circ} \mathrm{K}$ & lux & $\mathrm{Ix}$ \\
calorie & $\mathrm{cal}$ & lumen & $\mathrm{Im}$ \\
bar & $\mathrm{bar}$ & stilb & $\mathrm{sb}$ \\
hour & $\mathrm{h}$ & &
\end{tabular}

\section{Notes}

1. The symbols whose unit names are preceded by dots are those which had already been adopted by a decision of the CIPM.

2. The symbol for the stere, the unit of volume for firewood, shall be "st" and not "s", which had been previously assigned to it by the CIPM.

3. To indicate a temperature interval or difference, rather than a temperature, the word "degree" in full, or the abbreviation "deg", must be used.

\section{0th CGPM, 1954}

\section{Definition of the thermodynamic temperature scale $(C R, 79)^{\star}$}

\section{Resolution 3}

The 10th Conférence Générale des Poids et Mesures decides to define the thermodynamic temperature scale by choosing the triple point of water as the fundamental fixed point, and assigning to it the temperature 273.16 degrees Kelvin, exactly.
* The CGPM abrogated certain decisions on units and terminology, in particular: micron, degree absolute, and the terms "degree", and "deg", 13th CGPM, 1967/68 (Resolutions 7 and 3, see pp. 155 and 153, respectively), and the litre; 16th CGPM, 1979

(Resolution 6, see p. 159).
* The 13th CGPM in 1967 explicitly defined the kelvin (Resolution 4, see p. 154). 


\section{Definition of the standard atmosphere $(C R, 79)$}

\section{Resolution 4}

The 10th Conférence Générale des Poids et Mesures (CGPM), having noted that the definition of the standard atmosphere given by the 9th CGPM when defining the International Temperature Scale led some physicists to believe that this definition of the standard atmosphere was valid only for accurate work in thermometry,

declares that it adopts, for general use, the definition:

1 standard atmosphere $=1013250$ dynes per square centimetre,

i.e., 101325 newtons per square metre.

\section{Practical system of units $(\mathrm{CR}, 80)^{\star}$}

\section{Resolution 6}

In accordance with the wish expressed by the 9th Conférence Générale des Poids et Mesures (CGPM) in its Resolution 6 concerning the establishment of a practical system of units of measurement for international use, the 10th CGPM

decides to adopt as base units of the system, the following units:

length

metre

mass

kilogram

time

second

electric current

ampere

thermodynamic temperature

degree Kelvin

luminous intensity

candela

CIPM, 1956

\section{IDefinition of the unit of time (second) (PV, 25, 77)*}

\section{Resolution 1}

In virtue of the powers invested in it by Resolution 5 of the 10th Conférence Générale des Poids et Mesures, the Comité International des Poids et Mesures,

\section{considering}

1. that the 9th General Assembly of the International Astronomical Union (Dublin, 1955) declared itself in favour of linking the second to the tropical year,

2. that, according to the decisions of the 8th General Assembly of the International Astronomical Union (Rome, 1952), the second of ephemeris time (ET) is the fraction

$$
\frac{12960276813}{408986496} \times 10^{-9} \text { of the tropical year for } 1900 \text { January } 0 \text { at } 12 \mathrm{~h} \mathrm{ET} \text {, }
$$

\section{decides}

"The second is the fraction $1 / 31556925.9747$ of the tropical year for 1900 January 0 at 12 hours ephemeris time."
* The unit name "degree kelvin" was changed to "kelvin" in 1967 by the 13th CGPM (Resolution 3, see p. 153).

* This definition was abrogated in 1967 by the 13th CGPM (Resolution 1, see p. 153) 


\section{Système International d'Unités (PV, 25, 83)}

\section{Resolution 3}

The Comité International des Poids et Mesures,

\section{considering}

- the task entrusted to it by Resolution 6 of the 9th Conférence Générale des Poids et Mesures (CGPM) concerning the establishment of a practical system of units of measurement suitable for adoption by all countries adhering to the Metre Convention,

- the documents received from twenty-one countries in reply to the enquiry requested by the 9th CGPM,

- Resolution 6 of the 10th CGPM, fixing the base units of the system to be established,

\section{recommends}

1. that the name "Système International d'Unités" be given to the system founded on the base units adopted by the 10th CGPM, viz:

[This is followed by the list of the six base units with their symbols, reproduced in Resolution 12 of the 11th CGPM (1960)].

2. that the units listed in the table below be used, without excluding others which might be added later:

[This is followed by the table of units reproduced in paragraph 4 of Resolution 12 of the 11th CGPM (1960)].

11th CGPM, 1960

I Definition of the metre $(\mathrm{CR}, 85)^{*}$

\section{Resolution 6}

The 11th Conférence Générale des Poids et Mesures (CGPM),

\section{considering}

- that the international Prototype does not define the metre with an accuracy adequate for the present needs of metrology,

- that it is moreover desirable to adopt a natural and indestructible standard,

decides

1. The metre is the length equal to 1650763.73 wavelengths in vacuum of the radiation corresponding to the transition between the levels $2 p_{10}$ and $5 d_{5}$ of the krypton 86 atom.

2. The definition of the metre in force since 1889, based on the international Prototype of platinum-iridium, is abrogated.

3. The international Prototype of the metre sanctioned by the 1st CGPM in 1889 shall be kept at the BIPM under the conditions specified in 1889.

\section{Definition of the unit of time (second) $(C R, 86)^{*}$}

\section{Resolution 9}

The 11th Conférence Générale des Poids et Mesures (CGPM),

\section{considering}

- the powers given to the Comité International des Poids et Mesures (CIPM) by the 10th CGPM to define the fundamental unit of time,
* This definition was abrogated in 1983 by the 17th CGPM (Resolution 1, see p. 160).

* This definition was abrogated in 1967 by the 13th CGPM (Resolution 1, see p. 153). 
- the decision taken by the CIPM in 1956,

ratifies the following definition:

"The second is the fraction $1 / 31556925.9747$ of the tropical year for 1900 January 0 at 12 hours ephemeris time."

\section{Système International d'Unités $(\mathrm{CR}, 87)^{*}$}

\section{Resolution 12}

The 11th Conférence Générale des Poids et Mesures (CGPM),

\section{considering}

- Resolution 6 of the 10th CGPM, by which it adopted six base units on which to establish a practical system of measurement for international use:

$\begin{array}{lll}\text { length } & \text { metre } & \mathrm{m} \\ \text { mass } & \text { kilogram } & \mathrm{kg} \\ \text { time } & \text { second } & \mathrm{s} \\ \text { electric current } & \text { ampere } & \mathrm{A} \\ \text { thermodynamic temperature } & \text { degree Kelvin } & { }^{\circ} \mathrm{K} \\ \text { luminous intensity } & \text { candela } & \mathrm{cd}\end{array}$

- Resolution 3 adopted by the Comité International des Poids et Mesures (CIPM) in 1956 ,

- the recommendations adopted by the CIPM in 1958 concerning an abbreviation for the name of the system, and prefixes to form multiples and submultiples of the units,

\section{decides}

1. the system founded on the six base units above is called the "Système International d'Unités";

2. the international abbreviation of the name of the system is: $\mathrm{SI}$;

3. names of multiples and submultiples of the units are formed by means of the following prefixes:

\begin{tabular}{|c|c|c|c|c|c|}
\hline Multiplying factor & Prefix & Symbol & Multiplying factor & Prefix & Symbol \\
\hline $1000000000000=10^{12}$ & tera & $\mathrm{T}$ & $0.1=10^{-1}$ & deci & $d$ \\
\hline $1000000000=10^{9}$ & giga & G & $0.01=10^{-2}$ & centi & c \\
\hline $1000000=10^{6}$ & mega & $\mathrm{M}$ & $0.001=10^{-3}$ & milli & $\mathrm{m}$ \\
\hline $1000=10^{3}$ & kilo & $\mathrm{k}$ & $0.000001=10^{-6}$ & micro & $\mu$ \\
\hline $100=10^{2}$ & hecto & $\mathrm{h}$ & $0.000000001=10^{-9}$ & nano & $\mathrm{n}$ \\
\hline $10=10^{1}$ & deca & da & $0.000000000001=10^{-12}$ & pico & $\mathrm{p}$ \\
\hline
\end{tabular}

4. the units listed below are used in the system, without excluding others which might be added later.

\section{Supplementary units}

$\begin{array}{lll}\text { plane angle } & \text { radian } & \text { rad } \\ \text { solid angle } & \text { steradian } & \mathrm{sr}\end{array}$

\author{
* The CGPM later \\ abrogated certain of its \\ decisions and extended the \\ list of prefixes, see notes \\ below.
}

The name and symbol for the unit of thermodynamic temperature was modified by the 13th CGPM in 1967 (Resolution 3, see p. 153).

A seventh base unit, the mole, was adopted by the 14th CGPM in 1971 (Resolution 3, see p. 156).

Further prefixes were adopted by the 12th CGPM in 1964 (Resolution 8, see p. 152), the 15th CGPM in 1975 (Resolution 10, see p. 158) and the 19th CGPM in 1991 (Resolution 4, see p. 164).

The 20th CGPM in 1995 abrogated the class of supplementary units in the SI (Resolution 8, see p. 164). These are now considered as derived units. 


\section{Derived units}

\begin{tabular}{|c|c|c|c|c|}
\hline area & square metre & $\mathrm{m}^{2}$ & & The 13th CGPM in 1967 \\
\hline volume & cubic metre & $\mathrm{m}^{3}$ & & (Resolution 6, see p. 154) \\
\hline frequency & hertz & $\mathrm{Hz}$ & $1 / \mathrm{s}$ & should be added to the list. \\
\hline mass density (density) & kilogram per cubic metre & $\mathrm{kg} / \mathrm{m}^{3}$ & & In principle, this list of \\
\hline speed, velocity & metre per second & $\mathrm{m} / \mathrm{s}$ & & limit. \\
\hline angular velocity & radian per second & $\mathrm{rad} / \mathrm{s}$ & & \\
\hline acceleration & metre per second squared & $\mathrm{m} / \mathrm{s}^{2}$ & & \\
\hline angular acceleration & radian per second squared & $\mathrm{rad} / \mathrm{s}^{2}$ & & \\
\hline force & newton & $\mathrm{N}$ & $\mathrm{kg} \cdot \mathrm{m} / \mathrm{s}^{2}$ & \\
\hline pressure (mechanical stress) & newton per square metre & $\mathrm{N} / \mathrm{m}^{2}$ & & \\
\hline kinematic viscosity & square metre per second & $\mathrm{m}^{2} / \mathrm{s}$ & & \\
\hline dynamic viscosity & $\begin{array}{l}\text { newton-second per square } \\
\text { metre }\end{array}$ & $\mathrm{N} \cdot \mathrm{s} / \mathrm{m}^{2}$ & & $\begin{array}{l}\text { Modern practice is to use } \\
\text { the phrase "amount of } \\
\text { heat" rather than "quantity }\end{array}$ \\
\hline work, energy, quantity of heat & joule & $\mathrm{J}$ & $N \cdot m$ & of heat", because the word \\
\hline power & watt & W & $\mathrm{J} / \mathrm{s}$ & $\begin{array}{l}\text { quantity has a different } \\
\text { meaning in metrology. }\end{array}$ \\
\hline $\begin{array}{l}\text { quantity of electricity (side bar) } \\
\text { tension (voltage), } \\
\text { potential difference, } \\
\text { electromotive force }\end{array}$ & coulomb & C & $A \cdot s$ & $\begin{array}{l}\text { Modern practice is to use } \\
\text { the phrase "amount of } \\
\text { electricity" rather than } \\
\text { "quantity of electricity" }\end{array}$ \\
\hline electric field strength & volt per metre & $\mathrm{V} / \mathrm{m}$ & & (see note above). \\
\hline electric resistance & ohm & $\Omega$ & VIA & \\
\hline capacitance & farad & $\mathrm{F}$ & $A \cdot s / V$ & \\
\hline magnetic flux & weber & $\mathrm{Wb}$ & $V \cdot s$ & \\
\hline inductance & henry & $\mathrm{H}$ & $V \cdot s / A$ & \\
\hline magnetic flux density & tesla & $\mathrm{T}$ & $\mathrm{Wb} / \mathrm{m}^{2}$ & \\
\hline magnetic field strength & ampere per metre & $\mathrm{A} / \mathrm{m}$ & & \\
\hline magnetomotive force & ampere & $A$ & & \\
\hline luminous flux & lumen & $\operatorname{lm}$ & $\mathrm{cd} \cdot \mathrm{sr}$ & \\
\hline luminance & candela per square metre & $\mathrm{cd} / \mathrm{m}^{2}$ & & \\
\hline illuminance & $\operatorname{lux}$ & Ix & $\mathrm{Im} / \mathrm{m}^{2}$ & \\
\hline
\end{tabular}

I Cubic decimetre and litre $(C R, 88)$

\section{Resolution 13}

The 11th Conférence Générale des Poids et Mesures (CGPM),

\section{considering}

- that the cubic decimetre and the litre are unequal and differ by about 28 parts in $10^{6}$,

- that determinations of physical quantities which involve measurements of volume are being made more and more accurately, thus increasing the risk of confusion between the cubic decimetre and the litre,

requests the Comité International des Poids et Mesures to study the problem and submit its conclusions to the 12th CGPM. 
CIPM, 1961

I Cubic decimetre and litre (PV, 29, 34)

\section{Recommendation}

The Comite International des Poids et Mesures recommends that the results of accurate measurements of volume be expressed in units of the International System and not in litres.

CIPM, 1964

I Atomic and molecular frequency standards (PV, 32, 26 and CR, 93)

\section{Declaration}

The Comité International des Poids et Mesures,

empowered by Resolution 5 of the 12th Conférence Générale des Poids et Mesures to name atomic or molecular frequency standards for temporary use for time measurements in physics,

declares that the standard to be employed is the transition between the hyperfine levels $F=4, M=0$ and $F=3, M=0$ of the ground state ${ }^{2} S_{1 / 2}$ of the caesium 133 atom, unperturbed by external fields, and that the frequency of this transition is assigned the value 9192631770 hertz.

\section{2th CGPM, 1964}

I Atomic standard of frequency $(\mathrm{CR}, 93)$

\section{Resolution 5}

The 12th Conférence Générale des Poids et Mesures (CGPM),

\section{considering}

- that the 11th CGPM noted in its Resolution 10 the urgency, in the interests of accurate metrology, of adopting an atomic or molecular standard of time interval,

- that, in spite of the results already obtained with caesium atomic frequency standards, the time has not yet come for the CGPM to adopt a new definition of the second, base unit of the Système International d'Unités, because of the new and considerable improvements likely to be obtained from work now in progress,

considering also that it is not desirable to wait any longer before time measurements in physics are based on atomic or molecular frequency standards,

empowers the Comité International des Poids et Mesures to name the atomic or molecular frequency standards to be employed for the time being,

requests the organizations and laboratories knowledgeable in this field to pursue work connected with a new definition of the second. 


\section{Litre $(C R, 93)$}

\section{Resolution 6}

The 12th Conférence Générale des Poids et Mesures (CGPM),

considering Resolution 13 adopted by the 11th CGPM in 1960 and the Recommendation adopted by the Comité International des Poids et Mesures in 1961,

1. abrogates the definition of the litre given in 1901 by the 3rd CGPM,

2. declares that the word "litre" may be employed as a special name for the cubic decimetre,

3. recommends that the name litre should not be employed to give the results of highaccuracy volume measurements.

\section{Curie $(C R, 94)^{*}$}

\section{Resolution 7}

The 12th Conférence Générale des Poids et Mesures,

considering that the curie has been used for a long time in many countries as unit of activity for radionuclides,

recognizing that in the Système International d'Unités (SI), the unit of this activity is the second to the power of minus one $\left(\mathrm{s}^{-1}\right)$,

accepts that the curie be still retained, outside $\mathrm{SI}$, as unit of activity, with the value $3.7 \times 10^{10} \mathrm{~s}^{-1}$. The symbol for this unit is $\mathrm{Ci}$.

\section{SI prefixes femto and atto $(C R, 94)^{*}$}

\section{Resolution 8}

The 12th Conférence Générale des Poids et Mesures (CGPM)

decides to add to the list of prefixes for the formation of names of multiples and submultiples of units, adopted by the 11th CGPM, Resolution 12, paragraph 3 , the following two new prefixes:

\begin{tabular}{lll} 
Multiplying factor & Prefix & Symbol \\
\hline $10^{-15}$ & femto & $\mathrm{f}$ \\
$10^{-18}$ & atto & a
\end{tabular}

I Decimal multiples and submultiples of the unit of mass (PV, 35, 29 and Metrologia, 1968, 4, 45)

\section{Recommendation 2}

The Comité International des Poids et Mesures,

considering that the rule for forming names of decimal multiples and submultiples of the units of paragraph 3 of Resolution 12 of the 11th Conférence Générale des Poids et Mesures (CGPM) (1960) might be interpreted in different ways when applied to the unit of mass,

declares that the rules of Resolution 12 of the 11th CGPM apply to the kilogram in the following manner: the names of decimal multiples and submultiples of the unit of mass are formed by attaching prefixes to the word "gram".

* New prefixes were added by the 15th CGPM in 1975 (Resolution 10, see p. 158).
* The name "becquerel" (Bq) was adopted by the 15th CGPM in 1975 (Resolution 8, see p. 157) for the SI unit of activity: $1 \mathrm{Ci}=3.7 \times 10^{10} \mathrm{~Bq}$. 
13th CGPM, 1967/68

ISI unit of time (second) (CR, 103 and Metrologia, 1968, 4, 43)

\section{Resolution 1}

The 13th Conférence Générale des Poids et Mesures (CGPM), considering

- that the definition of the second adopted by the Comite International des Poids et Mesures (CIPM) in 1956 (Resolution 1) and ratified by Resolution 9 of the 11th CGPM (1960), later upheld by Resolution 5 of the 12th CGPM (1964), is inadequate for the present needs of metrology,

- that at its meeting of 1964 the CIPM, empowered by Resolution 5 of the 12th CGPM (1964), recommended, in order to fulfil these requirements, a caesium atomic frequency standard for temporary use,

- that this frequency standard has now been sufficiently tested and found sufficiently accurate to provide a definition of the second fulfilling present requirements,

- that the time has now come to replace the definition now in force of the unit of time of the Système International d'Unités by an atomic definition based on that standard,

\section{decides}

1. The SI unit of time is the second defined as follows:

"The second is the duration of 9192631770 periods of the radiation corresponding to the transition between the two hyperfine levels of the ground state of the caesium 133 atom";

2. Resolution 1 adopted by the CIPM at its meeting of 1956 and Resolution 9 of the 11th CGPM are now abrogated.

I SI unit of thermodynamic temperature (kelvin) (CR, 104 and Metrologia, $1968,4,43)^{*}$

\section{Resolution 3}

The 13th Conférence Générale des Poids et Mesures (CGPM),

\section{considering}

- the names "degree Kelvin" and "degree", the symbols " $\mathrm{K}$ " and "deg" and the rules for their use given in Resolution 7 of the 9th CGPM (1948), in Resolution 12 of the 11th CGPM (1960), and the decision taken by the Comité International des Poids et Mesures in 1962 (PV, 30, 27),

- that the unit of thermodynamic temperature and the unit of temperature interval are one and the same unit, which ought to be denoted by a single name and a single symbol,

\section{decides}

1. the unit of thermodynamic temperature is denoted by the name "kelvin" and its symbol is " $\mathrm{K}$ ";**

2. the same name and the same symbol are used to express a temperature interval;

3. a temperature interval may also be expressed in degrees Celsius;

4. the decisions mentioned in the opening paragraph concerning the name of the unit of thermodynamic temperature, its symbol and the designation of the unit to express an interval or a difference of temperatures are abrogated, but the usages which derive from these decisions remain permissible for the time being.

At its 1997 meeting, the CIPM affirmed that this definition refers to a caesium atom at rest at a thermodynamic temperature of $0 \mathrm{~K}$.

* At its 1980 meeting, the CIPM approved the report of the 7th meeting of the $\mathrm{CCU}$, which requested that the use of the symbols " $\mathrm{K}$ " and "deg" no longer be permitted.

** See Recommendation 2 (CI-2005) of the CIPM on the isotopic composition of water entering in the definition of the kelvin, p. 170. 
I Definition of the SI unit of thermodynamic temperature (kelvin) (CR, 104 and Metrologia, 1968, 4, 43)*

\section{Resolution 4}

The 13th Conférence Générale des Poids et Mesures (CGPM),

considering that it is useful to formulate more explicitly the definition of the unit of thermodynamic temperature contained in Resolution 3 of the 10th CGPM (1954),

decides to express this definition as follows:

"The kelvin, unit of thermodynamic temperature, is the fraction 1/273.16 of the thermodynamic temperature of the triple point of water."

- SI unit of luminous intensity (candela) (CR, 104 and Metrologia, 1968, 4, 43-44)*

\section{Resolution 5}

The 13th Conférence Générale des Poids et Mesures (CGPM),

\section{considering}

- the definition of the unit of luminous intensity ratified by the 9th CGPM (1948) and contained in the "Resolution concerning the change of photometric units" adopted by the Comité International des Poids et Mesures in 1946 (PV, 20, 119) in virtue of the powers conferred by the 8th CGPM (1933),

- that this definition fixes satisfactorily the unit of luminous intensity, but that its wording may be open to criticism,

decides to express the definition of the candela as follows:

"The candela is the luminous intensity, in the perpendicular direction, of a surface of $1 / 600000$ square metre of a black body at the temperature of freezing platinum under a pressure of 101325 newtons per square metre."

I SI derived units (CR, 105 and Metrologia, 1968, 4, 44)*

\section{Resolution 6}

The 13th Conférence Générale des Poids et Mesures (CGPM),

considering that it is useful to add some derived units to the list of paragraph 4 of Resolution 12 of the 11th CGPM (1960),

decides to add:

wave number

entropy

specific heat capacity

thermal conductivity

radiant intensity

activity (of a radioactive source)

1 per metre
joule per kelvin
joule per kilogram kelvin
watt per metre kelvin
watt per steradian
1 per second

1 per second

$\mathrm{m}^{-1}$
$\mathrm{~J} / \mathrm{K}$
$\mathrm{J} /(\mathrm{kg} \cdot \mathrm{K})$
$\mathrm{W} /(\mathrm{m} \cdot \mathrm{K})$
$\mathrm{W} / \mathrm{sr}$
$\mathrm{s}^{-1}$

$\mathrm{m}^{-1}$
* See Recommendation 5 (CI-1989) of the CIPM on the International Temperature Scale of 1990, p. 163.
* This definition was abrogated by the 16th CGPM in 1979 (Resolution 3, see p. 158)
* The unit of activity was given a special name and symbol by the 15 th CGPM in 1975 (Resolution 8, see p. 157). 
I Abrogation of earlier decisions (micron and new candle) (CR, 105 and Metrologia, 1968, 4, 44)

\section{Resolution 7}

The 13th Conférence Générale des Poids et Mesures (CGPM),

considering that subsequent decisions of the General Conference concerning the Système International d'Unités are incompatible with parts of Resolution 7 of the 9th CGPM (1948),

decides accordingly to remove from Resolution 7 of the 9th Conference:

1. the unit name "micron", and the symbol " $\mu$ " which had been given to that unit but which has now become a prefix;

2. the unit name "new candle".

CIPM, 1969

I Système International d'Unités, Rules for application of Resolution 12 of the 11th CGPM (1960) (PV, 37, 30 and Metrologia, 1970, 6, 66)*

\section{Recommendation 1}

The Comité International des Poids et Mesures,

considering that Resolution 12 of the 11th Conférence Générale des Poids et Mesures (CGPM) (1960), concerning the Système International d'Unités, has provoked discussions on certain of its aspects,

\section{declares}

1. the base units, the supplementary units and the derived units of the Système International d'Unités, which form a coherent set, are denoted by the name "SI units"; **

2. the prefixes adopted by the CGPM for the formation of decimal multiples and submultiples of SI units are called "SI prefixes";

\section{and recommends}

3. the use of SI units and of their decimal multiples and submultiples whose names are formed by means of SI prefixes.

Note: The name "supplementary units", appearing in Resolution 12 of the 11th CGPM (and in the present Recommendation) is given to SI units for which the General Conference declines to state whether they are base units or derived units.

CCDS, 1970 (In CIPM, 1970)

IDefinition of TAI (PV, 38, 110-111 and Metrologia, 1971, 7, 43)

\section{Recommendation S 2}

International Atomic Time (TAl) is the time reference coordinate established by the Bureau International de l'Heure on the basis of the readings of atomic clocks operating in various establishments in accordance with the definition of the second, the unit of time of the International System of Units.

In 1980, the definition of TAI was completed as follows (declaration of the CCDS, BIPM Com. Cons. Déf. Seconde, 1980, 9, S 15 and Metrologia, 1981, 17, 70):

$\mathrm{TAl}$ is a coordinate time scale defined in a geocentric reference frame with the SI second as realized on the rotating geoid as the scale unit.

This definition was further amplified by the International Astronomical

Union in 1991,

Resolution A4:

"TAI is a realized time scale whose ideal form, neglecting a constant offset of $32.184 \mathrm{~s}$, is Terrestrial Time (TT), itself related to the time coordinate of the geocentric reference frame, Geocentric Coordinate Time (TCG), by a constant rate."

(see Proc. 21st General Assembly of the IAU, IAU Trans., 1991, vol. XXIB, Kluwer.) 


\section{4th CGPM, 1971}

\section{Pascal and siemens $(\mathrm{CR}, 78)$}

The 14th Conférence Générale des Poids et Mesures adopted the special names "pascal" (symbol Pa), for the SI unit newton per square metre, and "siemens" (symbol S), for the SI unit of electric conductance [reciprocal ohm].

I International Atomic Time, function of CIPM (CR, 77-78 and Metrologia, 1972, 8, 35)

\section{Resolution 1}

The 14th Conférence Générale des Poids et Mesures (CGPM),

\section{considering}

- that the second, unit of time of the Système International d'Unités, has since 1967 been defined in terms of a natural atomic frequency, and no longer in terms of the time scales provided by astronomical motions,

- that the need for an International Atomic Time (TAI) scale is a consequence of the atomic definition of the second,

- that several international organizations have ensured and are still successfully ensuring the establishment of the time scales based on astronomical motions, particularly thanks to the permanent services of the Bureau International de l'Heure $(\mathrm{BIH})$,

- that the BIH has started to establish an atomic time scale of recognized quality and proven usefulness,

- that the atomic frequency standards for realizing the second have been considered and must continue to be considered by the Comité International des Poids et Mesures (CIPM) helped by a Consultative Committee, and that the unit interval of the International Atomic Time scale must be the second realized according to its atomic definition,

- that all the competent international scientific organizations and the national laboratories active in this field have expressed the wish that the CIPM and the CGPM should give a definition of International Atomic Time, and should contribute to the establishment of the International Atomic Time scale,

- that the usefulness of International Atomic Time entails close coordination with the time scales based on astronomical motions,

\section{requests the CIPM}

1. to give a definition of International Atomic Time,

2. to take the necessary steps, in agreement with the international organizations concerned, to ensure that available scientific competence and existing facilities are used in the best possible way to realize the International Atomic Time scale and to satisfy the requirements of users of International Atomic Time.

I SI unit of amount of substance (mole) (CR, 78 and Metrologia, 1972, 8, 36)*

\section{Resolution 3}

The 14th Conférence Générale des Poids et Mesures (CGPM),

considering the advice of the International Union of Pure and Applied Physics, of the International Union of Pure and Applied Chemistry, and of the International Organization for Standardization, concerning the need to define a unit of amount of substance,

The definition of TAI was given by the CCDS in 1970 (now the CCTF), see p. 155.

* At its 1980 meeting, the CIPM approved the report of the 7th meeting of the CCU (1980) specifying that, in this definition, it is understood that unbound atoms of carbon 12, at rest and in their ground state, are referred to. 


\section{decides}

1. The mole is the amount of substance of a system which contains as many elementary entities as there are atoms in 0.012 kilogram of carbon 12; its symbol is "mol".

2. When the mole is used, the elementary entities must be specified and may be atoms, molecules, ions, electrons, other particles, or specified groups of such particles.

3. The mole is a base unit of the Système International d'Unités.

\section{5th CGPM, 1975}

- Recommended value for the speed of light (CR, 103 and Metrologia, 1975, $11,179-180)$

\section{Resolution 2}

The 15th Conférence Générale des Poids et Mesures,

considering the excellent agreement among the results of wavelength measurements on the radiations of lasers locked on a molecular absorption line in the visible or infrared region, with an uncertainty estimated at $\pm 4 \times 10^{-9}$ which corresponds to the uncertainty of the realization of the metre,

considering also the concordant measurements of the frequencies of several of these radiations,

recommends the use of the resulting value for the speed of propagation of electromagnetic waves in vacuum $c=299792458$ metres per second.

I Coordinated Universal Time (UTC) (CR, 104 and Metrologia, 1975, 11, 180)

\section{Resolution 5}

The 15th Conférence Générale des Poids et Mesures,

considering that the system called "Coordinated Universal Time" (UTC) is widely used, that it is broadcast in most radio transmissions of time signals, that this wide diffusion makes available to the users not only frequency standards but also International Atomic Time and an approximation to Universal Time (or, if one prefers, mean solar time),

notes that this Coordinated Universal Time provides the basis of civil time, the use of which is legal in most countries,

judges that this usage can be strongly endorsed.

I SI units for ionizing radiation (becquerel and gray) (CR, 105 and Metrologia, 1975, 11, 180)*

\section{Resolutions 8 and 9}

The 15th Conférence Générale des Poids et Mesures,

by reason of the pressing requirement, expressed by the International Commission on Radiation Units and Measurements (ICRU), to extend the use of the Systeme International d'Unités to radiological research and applications,

The relative uncertainty given here corresponds to three standard deviations in the data considered.

by reason of the need to make as easy as possible the use of the units for nonspecialists, taking into consideration also the grave risks of errors in therapeutic work, adopts the following special name for the SI unit of activity: becquerel, symbol Bq, equal to one reciprocal second (Resolution 8), adopts the following special name for the SI unit of ionizing radiation: gray, symbol Gy, equal to one joule per kilogram (Resolution 9). 
Note: The gray is the SI unit of absorbed dose. In the field of ionizing radiation, the gray may be used with other physical quantities also expressed in joules per kilogram: the Comité Consultatif des Unités has responsibility for studying this matter in collaboration with the competent international organizations.

I SI prefixes peta and exa (CR, 106 and Metrologia, 1975, 11, 180-181)*

\section{Resolution 10}

The 15th Conférence Générale des Poids et Mesures (CGPM)

decides to add to the list of SI prefixes to be used for multiples, which was adopted by the 11th CGPM, Resolution 12, paragraph 3, the two following prefixes:

\begin{tabular}{lll} 
Multiplying factor & Prefix & Symbol \\
\hline $10^{15}$ & peta & P \\
$10^{18}$ & exa & E
\end{tabular}

\section{6th CGPM, 1979}

I SI unit of luminous intensity (candela) (CR, 100 and Metrologia, 1980, 16, 56)

\section{Resolution 3}

The 16th Conférence Générale des Poids et Mesures (CGPM),

\section{considering}

- that despite the notable efforts of some laboratories there remain excessive divergences between the results of realizations of the candela based upon the present black body primary standard,

- that radiometric techniques are developing rapidly, allowing precisions that are already equivalent to those of photometry and that these techniques are already in use in national laboratories to realize the candela without having to construct a black body,

- that the relation between luminous quantities of photometry and radiometric quantities, namely the value of 683 lumens per watt for the spectral luminous efficacy of monochromatic radiation of frequency $540 \times 10^{12}$ hertz, has been adopted by the Comité International des Poids et Mesures (CIPM) in 1977,

- that this value has been accepted as being sufficiently accurate for the system of luminous photopic quantities, that it implies a change of only about $3 \%$ for the system of luminous scotopic quantities, and that it therefore ensures satisfactory continuity,

- that the time has come to give the candela a definition that will allow an improvement in both the ease of realization and the precision of photometric standards, and that applies to both photopic and scotopic photometric quantities and to quantities yet to be defined in the mesopic field,

\section{decides}

1. The candela is the luminous intensity, in a given direction, of a source that emits monochromatic radiation of frequency $540 \times 10^{12}$ hertz and that has a radiant intensity in that direction of $1 / 683$ watt per steradian.

2. The definition of the candela (at the time called new candle) adopted by the CIPM in 1946 by reason of the powers conferred by the 8th CGPM in 1933, ratified by the 9th CGPM in 1948, then amended by the 13th CGPM in 1967, is abrogated.
* New prefixes were added by the 19th CGPM in 1991 (Resolution 4, see p. 164).
Photopic vision is detected by the cones on the retina of the eye, which are sensitive to a high level of luminance

$\left(\mathrm{L}>\right.$ ca. $\left.10 \mathrm{~cd} / \mathrm{m}^{2}\right)$ and are used in daytime vision.

Scotopic vision is detected by the rods of the retina, which are sensitive to low level luminance $\left(\mathrm{L}<\right.$ ca. $10^{-3} \mathrm{~cd} / \mathrm{m}^{2}$, used in night vision. In the domain between these levels of luminance both cones and rods are used, and this is described as mesopic vision. 
I Special name for the SI unit of dose equivalent (sievert) (CR, 100 and Metrologia, 1980, 16, 56)*

\section{Resolution 5}

The 16th Conférence Générale des Poids et Mesures,

\section{considering}

- the effort made to introduce SI units into the field of ionizing radiations,

- the risk to human beings of an underestimated radiation dose, a risk that could result from a confusion between absorbed dose and dose equivalent,

- that the proliferation of special names represents a danger for the Système International d'Unités and must be avoided in every possible way, but that this rule can be broken when it is a matter of safeguarding human health,

adopts the special name sievert, symbol Sv, for the SI unit of dose equivalent in the field of radioprotection. The sievert is equal to the joule per kilogram.

I Symbols for the litre (CR, 101 and Metrologia, 1980, 16, 56-57)

\section{Resolution 6}

The 16th Conférence Générale des Poids et Mesures (CGPM),

recognizing the general principles adopted for writing the unit symbols in Resolution 7 of the 9th CGPM (1948),

considering that the symbol I for the unit litre was adopted by the Comite International des Poids et Mesures (CIPM) in 1879 and confirmed in the same Resolution of 1948,

considering also that, in order to avoid the risk of confusion between the letter I and the number 1 , several countries have adopted the symbol $L$ instead of I for the unit litre,

considering that the name litre, although not included in the Système International d'Unités, must be admitted for general use with the System,

decides, as an exception, to adopt the two symbols I and $L$ as symbols to be used for the unit litre,

considering further that in the future only one of these two symbols should be retained,

invites the CIPM to follow the development of the use of these two symbols and to give the 18th CGPM its opinion as to the possibility of suppressing one of them.

\section{CIPM, 1980}

I SI supplementary units (radian and steradian) (PV, 48, 24 and Metrologia, $1981,17,72)^{*}$

\section{Recommendation 1}

The Comité International des Poids et Mesures (CIPM),

taking into consideration Resolution 3 adopted by ISO/TC 12 in 1978 and Recommendation U 1 (1980) adopted by the Comité Consultatif des Unités at its 7th meeting,

\section{considering}

- that the units radian and steradian are usually introduced into expressions for units when there is need for clarification, especially in photometry where the steradian plays an important role in distinguishing between units corresponding to different quantities,

- that in the equations used one generally expresses plane angle as the ratio of two lengths and solid angle as the ratio between an area and the square of a length, and consequently that these quantities are treated as dimensionless quantities,
* The CIPM, in 1984, decided to accompany this Resolution with an explanation

(Recommendation 1, see p. 161)

The CIPM, in 1990, considered that it was still too early to choose a single symbol for the litre.
* The class of SI supplementary units was abrogated by decision of the 20th CGPM in 1995 (Resolution 8, see p. 164). 
- that the study of the formalisms in use in the scientific field shows that none exists which is at the same time coherent and convenient and in which the quantities plane angle and solid angle might be considered as base quantities,

\section{considering also}

- that the interpretation given by the CIPM in 1969 for the class of supplementary units introduced in Resolution 12 of the 11th Conférence Générale des Poids et Mesures (CGPM) in 1960 allows the freedom of treating the radian and the steradian as SI base units,

- that such a possibility compromises the internal coherence of the SI based on only seven base units,

decides to interpret the class of supplementary units in the International System as a class of dimensionless derived units for which the CGPM allows the freedom of using or not using them in expressions for SI derived units.

\section{7th CGPM, 1983}

IDefinition of the metre (CR, 97 and Metrologia, 1984, 20, 25)

\section{Resolution 1}

The 17th Conférence Générale des Poids et Mesures (CGPM),

\section{considering}

- that the present definition does not allow a sufficiently precise realization of the metre for all requirements,

- that progress made in the stabilization of lasers allows radiations to be obtained that are more reproducible and easier to use than the standard radiation emitted by a krypton 86 lamp,

- that progress made in the measurement of the frequency and wavelength of these radiations has resulted in concordant determinations of the speed of light whose accuracy is limited principally by the realization of the present definition of the metre,

- that wavelengths determined from frequency measurements and a given value for the speed of light have a reproducibility superior to that which can be obtained by comparison with the wavelength of the standard radiation of krypton 86,

- that there is an advantage, notably for astronomy and geodesy, in maintaining unchanged the value of the speed of light recommended in 1975 by the 15th CGPM in its Resolution 2 ( $c=299792458 \mathrm{~m} / \mathrm{s})$,

- that a new definition of the metre has been envisaged in various forms all of which have the effect of giving the speed of light an exact value, equal to the recommended value, and that this introduces no appreciable discontinuity into the unit of length, taking into account the relative uncertainty of $\pm 4 \times 10^{-9}$ of the best realizations of the present definition of the metre,

- that these various forms, making reference either to the path travelled by light in a specified time interval or to the wavelength of a radiation of measured or specified frequency, have been the object of consultations and deep discussions, have been recognized as being equivalent and that a consensus has emerged in favour of the first form,

- that the Comité Consultatif pour la Définition du Mètre (CCDM) is now in a position to give instructions for the practical realization of such a definition, instructions which could include the use of the orange radiation of krypton 86 used as standard up to now, and which may in due course be extended or revised,

The relative uncertainty given here corresponds to three standard deviations in the data considered. 


\section{decides}

1. The metre is the length of the path travelled by light in vacuum during a time interval of $1 / 299792458$ of a second,

2. The definition of the metre in force since 1960, based upon the transition between the levels $2 p_{10}$ and $5 d_{5}$ of the atom of krypton 86 , is abrogated.

I On the realization of the definition of the metre (CR, 98 and Metrologia, 1984, 20, 25-26)

\section{Resolution 2}

The 17th Conférence Générale des Poids et Mesures,

invites the Comité International des Poids et Mesures

- to draw up instructions for the practical realization of the new definition of the metre,

- to choose radiations which can be recommended as standards of wavelength for the interferometric measurement of length and to draw up instructions for their use,

- to pursue studies undertaken to improve these standards.

\section{CIPM, 1984}

I Concerning the sievert (PV, 52, 31 and Metrologia, 1985, 21, 90)*

\section{Recommendation 1}

The Comité International des Poids et Mesures,

considering the confusion which continues to exist on the subject of Resolution 5, approved by the 16th Conférence Générale des Poids et Mesures (1979),

decides to introduce the following explanation in the brochure "Le Système International d'Unités (SI)":

The quantity dose equivalent $H$ is the product of the absorbed dose $D$ of ionizing radiation and the dimensionless factors $Q$ (quality factor) and $N$ (product of any other multiplying factors) stipulated by the International Commission on Radiological Protection:

$$
H=Q \cdot N \cdot D \text {. }
$$

Thus, for a given radiation, the numerical value of $H$ in joules per kilogram may differ from that of $D$ in joules per kilogram depending upon the values of $Q$ and $N$. In order to avoid any risk of confusion between the absorbed dose $D$ and the dose equivalent $H$, the special names for the respective units should be used, that is, the name gray should be used instead of joules per kilogram for the unit of absorbed dose $D$ and the name sievert instead of joules per kilogram for the unit of dose equivalent $H$.

\section{8th CGPM, 1987}

I Forthcoming adjustment to the representations of the volt and of the ohm (CR, 100 and Metrologia, 1988, 25, 115)

\section{Resolution 6}

The 18th Conférence Générale des Poids et Mesures, considering

- that worldwide uniformity and long-term stability of national representations of the electrical units are of major importance for science, commerce and industry from both the technical and economic points of view,
See Recommendation 1 (CI-2002) of the CIPM on the revision of the practical realization of the definition of the metre, p. 166.

* The CIPM, in 2002, decided to change the explanation of the quantity dose equivalent in the SI Brochure (Recommendation 2, see p. 168). 
- that many national laboratories use the Josephson effect and are beginning to use the quantum Hall effect to maintain, respectively, representations of the volt and of the ohm, as these offer the best guarantees of long-term stability,

- that because of the importance of coherence among the units of measurement of the various physical quantities the values adopted for these representations must be as closely as possible in agreement with the $\mathrm{SI}$,

- that the results of recent and current experiment will permit the establishment of an acceptable value, sufficiently compatible with the SI, for the coefficient which relates each of these effects to the corresponding electrical unit,

invites the laboratories whose work can contribute to the establishment of the quotient voltage/frequency in the case of the Josephson effect and of the quotient voltage/current for the quantum Hall effect to vigorously pursue these efforts and to communicate their results without delay to the Comité International des Poids et Mesures, and

instructs the Comité International des Poids et Mesures to recommend, as soon as it considers it possible, a value for each of these quotients together with a date for them to be put into practice simultaneously in all countries; these values should be announced at least one year in advance and would be adopted on 1 January 1990.

\section{CIPM, 1988}

I Representation of the volt by means of the Josephson effect (PV, 56, 44 and Metrologia, 1989, 26, 69)

\section{Recommendation 1}

The Comité International des Poids et Mesures,

acting in accordance with instructions given in Resolution 6 of the 18th Conference Générale des Poids et Mesures concerning the forthcoming adjustment of the representations of the volt and the ohm,

\section{considering}

- that a detailed study of the results of the most recent determinations leads to a value of $483597.9 \mathrm{GHz} / \mathrm{V}$ for the Josephson constant, $K_{J}$, that is to say, for the quotient of frequency divided by the potential difference corresponding to the $n=1$ step in the Josephson effect,

- that the Josephson effect, together with this value of $K_{\mathrm{J}}$, can be used to establish a reference standard of electromotive force having a one-standard-deviation uncertainty with respect to the volt estimated to be 4 parts in $10^{7}$, and a reproducibility which is significantly better,

\section{recommends}

- that $483597.9 \mathrm{GHz} / \mathrm{N}$ exactly be adopted as a conventional value, denoted by $K_{\mathrm{J}-90}$ for the Josephson constant, $K_{\mathrm{J}}$,

- that this new value be used from 1 January 1990, and not before, to replace the values currently in use,

- that this new value be used from this same date by all laboratories which base their measurements of electromotive force on the Josephson effect, and

- that from this same date all other laboratories adjust the value of their laboratory reference standards to agree with the new adopted value,

is of the opinion that no change in this recommended value of the Josephson constant will be necessary in the foreseeable future, and

draws the attention of laboratories to the fact that the new value is greater by $3.9 \mathrm{GHz} / \mathrm{V}$, or about 8 parts in $10^{6}$, than the value given in 1972 by the Comité Consultatif d'Électricité in its Declaration E-72. 
I Representation of the ohm by means of the quantum Hall effect ( $P V, 56$, 45 and Metrologia, 1989, 26, 70)

\section{Recommendation 2}

The Comité International des Poids et Mesures,

acting in accordance with instructions given in Resolution 6 of the 18th Conference Générale des Poids et Mesures concerning the forthcoming adjustment of the representations of the volt and the ohm,

\section{considering}

- that most existing laboratory reference standards of resistance change significantly with time,

- that a laboratory reference standard of resistance based on the quantum Hall effect would be stable and reproducible,

- that a detailed study of the results of the most recent determinations leads to a value of $25812.807 \Omega$ for the von Klitzing constant, $R_{\mathrm{K}}$, that is to say, for the quotient of the Hall potential difference divided by current corresponding to the plateau $i=1$ in the quantum Hall effect,

- that the quantum Hall effect, together with this value of $R_{\mathrm{K}}$, can be used to establish a reference standard of resistance having a one-standard-deviation uncertainty with respect to the ohm estimated to be 2 parts in $10^{7}$, and a reproducibility which is significantly better,

\section{recommends}

- that $25812.807 \Omega$ exactly be adopted as a conventional value, denoted by $R_{\mathrm{K}-90}$, for the von Klitzing constant, $R_{\mathrm{K}}$,

- that this value be used from 1 January 1990, and not before, by all laboratories which base their measurements of resistance on the quantum Hall effect,

- that from this same date all other laboratories adjust the value of their laboratory reference standards to agree with $R_{\mathrm{K}-90}$,

- that in the use of the quantum Hall effect to establish a laboratory reference standard of resistance, laboratories follow the most recent edition of the technical guidelines for reliable measurements of the quantized Hall resistance drawn up by the Comité Consultatif d'Électricité and published by the Bureau International des Poids et Mesures, and

is of the opinion that no change in this recommended value of the von Klitzing constant will be necessary in the foreseeable future.

\section{CIPM, 1989}

I The International Temperature Scale of 1990 (PV, 57, 115 and Metrologia, 1990, 27, 13)

\section{Recommendation 5}

The Comité International des Poids et Mesures (CIPM) acting in accordance with Resolution 7 of the 18th Conférence Générale des Poids et Mesures (1987) has adopted the International Temperature Scale of 1990 (ITS-90) to supersede the International Practical Temperature Scale of 1968 (IPTS-68).

The CIPM notes that, by comparison with the IPTS-68, the ITS-90

- extends to lower temperatures, down to $0.65 \mathrm{~K}$, and hence also supersedes the EPT-76,

- is in substantially better agreement with corresponding thermodynamic temperatures,
At its 89th meeting in 2000, the CIPM approved the declaration of the 22nd meeting of the CCEM on the use of the value of the von Klitzing constant, see p. 166. 
- has much improved continuity, precision and reproducibility throughout its range and

- has subranges and alternative definitions in certain ranges which greatly facilitate its use.

The CIPM also notes that, to accompany the text of the ITS-90 there will be two further documents, the Supplementary Information for the ITS-90 and Techniques for Approximating the ITS-90. These documents will be published by the BIPM and periodically updated.

The CIPM recommends

- that on 1 January 1990 the ITS-90 come into force and

- that from this same date the IPTS-68 and the EPT-76 be abrogated.

\section{9th CGPM, 1991}

I SI prefixes zetta, zepto, yotta and yocto (CR, 185 and Metrologia, 1992, 29, 3)

\section{Resolution 4}

The 19th Conférence Générale des Poids et Mesures (CGPM)

decides to add to the list of SI prefixes to be used for multiples and submultiples of units, adopted by the 11th CGPM, Resolution 12, paragraph 3, the 12th CGPM, Resolution 8 and the 15th CGPM, Resolution 10, the following prefixes:

\begin{tabular}{lll} 
Multiplying factor & Prefix & Symbol \\
\hline $10^{21}$ & zetta & $\mathrm{Z}$ \\
$10^{-21}$ & zepto & $\mathrm{Z}$ \\
$10^{24}$ & yotta & $\mathrm{Y}$ \\
$10^{-24}$ & yocto & $\mathrm{y}$
\end{tabular}

The names zepto and zetta are derived from septo suggesting the number seven (the seventh power of $10^{3}$ ) and the letter " $z$ " is substituted for the letter "s" to avoid the duplicate use of the letter "s" as a symbol. The names yocto and yotta are derived from octo, suggesting the number eight (the eighth power of $10^{3}$ ); the letter " $y$ " is added to avoid the use of the letter "o" as a symbol because it may be confused with the number zero.

\section{0th CGPM, 1995}

I Elimination of the class of supplementary units in the SI (CR, 223 and Metrologia, 1996, 33, 83)

\section{Resolution 8}

The 20th Conférence Générale des Poids et Mesures (CGPM),

\section{considering}

- that the 11th Conférence Générale in 1960 in its Resolution 12, establishing the Système International d'Unités, SI, distinguished between three classes of SI units: the base units, the derived units, and the supplementary units, the last of these comprising the radian and the steradian,

- that the status of the supplementary units in relation to the base units and the derived units gave rise to debate,

- that the Comité International des Poids et Mesures, in 1980, having observed that the ambiguous status of the supplementary units compromises the internal coherence of the $\mathrm{SI}$, has in its Recommendation $1(\mathrm{Cl}-1980)$ interpreted the supplementary units, in the $\mathrm{SI}$, as dimensionless derived units,

approving the interpretation given by the Comite International in 1980, 


\section{decides}

- to interpret the supplementary units in the SI, namely the radian and the steradian, as dimensionless derived units, the names and symbols of which may, but need not, be used in expressions for other SI derived units, as is convenient,

- and, consequently, to eliminate the class of supplementary units as a separate class in the SI.

\section{1st CGPM, 1999}

I The definition of the kilogram (CR, 331 and Metrologia, 2000, 37, 94)

\section{Resolution 7}

The 21st Conférence Générale des Poids et Mesures,

\section{considering}

- the need to assure the long-term stability of the International System of Units (SI),

- the intrinsic uncertainty in the long-term stability of the artefact defining the unit of mass, one of the base units of the SI,

- the consequent uncertainty in the long-term stability of the other three base units of the SI that depend on the kilogram, namely, the ampere, the mole and the candela,

- the progress already made in a number of different experiments designed to link the unit of mass to fundamental or atomic constants,

- the desirability of having more than one method of making such a link,

recommends that national laboratories continue their efforts to refine experiments that link the unit of mass to fundamental or atomic constants with a view to a future redefinition of the kilogram.

\section{Special name for the SI derived unit mole per second, the katal, for the expression of catalytic activity (CR, 334-335 and Metrologia, 2000, 37, 95)}

\section{Resolution 12}

The 21st Conférence Générale des Poids et Mesures,

\section{considering}

- the importance for human health and safety of facilitating the use of SI units in the fields of medicine and biochemistry,

- that a non-SI unit called "unit", symbol $\mathrm{U}$, equal to $1 \mu \mathrm{mol} \cdot \mathrm{min}^{-1}$, which is not coherent with the International System of Units (SI), has been in widespread use in medicine and biochemistry since 1964 for expressing catalytic activity,

- that the absence of a special name for the SI coherent derived unit mole per second has led to results of clinical measurements being given in various local units,

- that the use of SI units in medicine and clinical chemistry is strongly recommended by the international unions in these fields,

- that the International Federation of Clinical Chemistry and Laboratory Medicine has asked the Consultative Committee for Units to recommend the special name katal, symbol kat, for the SI unit mole per second,

- that while the proliferation of special names represents a danger for the SI, exceptions are made in matters related to human health and safety (15th General Conference, 1975, Resolutions 8 and 9, 16th General Conference, 1979, Resolution 5), 
noting that the name katal, symbol kat, has been used for the SI unit mole per second for over thirty years to express catalytic activity,

decides to adopt the special name katal, symbol kat, for the SI unit mole per second to express catalytic activity, especially in the fields of medicine and biochemistry,

and recommends that when the katal is used, the measurand be specified by reference to the measurement procedure; the measurement procedure must identify the indicator reaction.

CIPM, 2000

I "use of the von Klitzing constant to express the value of a reference standard of resistance as a function of quantum Hall effect" (PV, 68, 101)

At its 89th meeting in 2000, the CIPM approved the following declaration of the 22nd meeting of the CCEM (CCEM, 22, 90):

"The CCEM, having reviewed the 1998 CODATA least squares adjustment of the fundamental constants, is now of the opinion that the quantum Hall effect, together with the value of $R_{\mathrm{K}-90}$, can be used to establish a reference standard of resistance having a relative one standard deviation uncertainty with respect to the ohm, estimated to be $1 \times 10^{-7}$, and a reproducibility which is significantly better. This represents a reduction in the uncertainty of a factor of two compared with the 1988 recommendation."

CIPM, 2001

I "SI units" and "units of the SI" (PV, 69, 120)

The CIPM approved in 2001 the following proposal of the CCU regarding "SI units" and "units of the SI":

"We suggest that "SI units" and "units of the SI" should be regarded as names that include both the base units and the coherent derived units, and also all units obtained by combining these with the recommended multiple and sub-multiple prefixes.

We suggest that the name "coherent SI units" should be used when it is desired to restrict the meaning to only the base units and the coherent derived units."

CIPM, 2002

I Revision of the practical realization of the definition of the metre (PV, 70, 194-204 and Metrologia, 40, 103-133)

\section{Recommendation 1}

The International Committee for Weights and Measures, recalling

- that in 1983 the 17th General Conference (CGPM) adopted a new definition of the metre;

- that in the same year the CGPM invited the International Committee (CIPM)

- to draw up instructions for the practical realization of the metre,

- to choose radiations which can be recommended as standards of wavelength for the interferometric measurement of length and draw up instructions for their use,

- to pursue studies undertaken to improve these standards and in due course to extend or revise these instructions; 
- that in response to this invitation the CIPM adopted Recommendation 1 (Cl-1983) (mise en pratique of the definition of the metre) to the effect

- that the metre should be realized by one of the following methods:

(a) by means of the length / of the path travelled in vacuum by a plane electromagnetic wave in a time $t$; this length is obtained from the measured time $t$, using the relation $I=c_{0} \cdot t$ and the value of the speed of light in vacuum $c_{0}=299792458 \mathrm{~m} / \mathrm{s}$,

(b) by means of the wavelength in vacuum $\lambda$ of a plane electromagnetic wave of frequency $f$; this wavelength is obtained from the measured frequency $f$ using the relation $\lambda=c_{0} / f$ and the value of the speed of light in vacuum $c_{0}=299792458 \mathrm{~m} / \mathrm{s}$,

(c) by means of one of the radiations from the list below, whose stated wavelength in vacuum or whose stated frequency can be used with the uncertainty shown, provided that the given specifications and accepted good practice are followed;

- that in all cases any necessary corrections be applied to take account of actual conditions such as diffraction, gravitation or imperfection in the vacuum;

- that in the context of general relativity, the metre is considered a unit of proper length. Its definition, therefore, applies only within a spatial extent sufficiently small that the effects of the non-uniformity of the gravitational field can be ignored (note that, at the surface of the Earth, this effect in the vertical direction is about 1 part in $10^{16}$ per metre). In this case, the effects to be taken into account are those of special relativity only. The local methods for the realization of the metre recommended in (b) and (c) provide the proper metre but not necessarily that given in (a). Method (a) should therefore be restricted to lengths / which are sufficiently short for the effects predicted by general relativity to be negligible with respect to the uncertainties of realization. For advice on the interpretation of measurements in which this is not the case, see the report of the Consultative Committee for Time and Frequency (CCTF) Working Group on the Application of General Relativity to Metrology (Application of general relativity to metrology, Metrologia, 1997, 34, 261290);

- that the CIPM had already recommended a list of radiations for this purpose;

recalling also that in 1992 and in 1997 the CIPM revised the practical realization of the definition of the metre;

\section{considering}

- that science and technology continue to demand improved accuracy in the realization of the metre;

- that since 1997 work in national laboratories, in the BIPM and elsewhere has identified new radiations and methods for their realization which lead to lower uncertainties;

- that there is an increasing move towards optical frequencies for time-related activities, and that there continues to be a general widening of the scope of application of the recommended radiations of the mise en pratique to cover not only dimensional metrology and the realization of the metre, but also high-resolution spectroscopy, atomic and molecular physics, fundamental constants and telecommunication;

- that a number of new frequency values with reduced uncertainties for radiations of highstability cold atom and ion standards already listed in the recommended radiations list are now available, that the frequencies of radiations of several new cold atom and ion species have also recently been measured, and that new improved values with substantially reduced uncertainties for a number of optical frequency standards based on gas cells have been determined, including the wavelength region of interest to optical telecommunications;

- that new femtosecond comb techniques have clear significance for relating the frequency of high-stability optical frequency standards to that of the frequency standard realizing the SI second, that these techniques represent a convenient measurement technique for providing traceability to the International System of Units (SI) and that 
comb technology also can provide frequency sources as well as a measurement technique;

recognizes comb techniques as timely and appropriate, and recommends further research to fully investigate the capability of the techniques;

welcomes validations now being made of comb techniques by comparison with other frequency chain techniques;

urges national metrology institutes and other laboratories to pursue the comb technique to the highest level of accuracy achievable and also to seek simplicity so as to encourage widespread application;

\section{recommends}

- that the list of recommended radiations given by the CIPM in 1997 (Recommendation $1(\mathrm{Cl}-1997))$ be replaced by the list of radiations given below*, including

- updated frequency values for cold $\mathrm{Ca}$ atom, $\mathrm{H}$ atom and the trapped $\mathrm{Sr}^{+}$ion,

- frequency values for new cold ion species including trapped $\mathrm{Hg}^{+}$ion, trapped $\mathrm{In}^{+}$ion and trapped $\mathrm{Yb}^{+}$ion,

- updated frequency values for Rb-stabilized lasers, $\mathrm{I}_{2}$-stabilized $\mathrm{Nd}$ :YAG and $\mathrm{He}-\mathrm{Ne}$ lasers, $\mathrm{CH}_{4}$-stabilized $\mathrm{He}$-Ne lasers and $\mathrm{OsO}_{4}$-stabilized $\mathrm{CO}_{2}$ lasers at $10 \mu \mathrm{m}$,

- frequency values for standards relevant to the optical communications bands, including $\mathrm{Rb}$ - and $\mathrm{C}_{2} \mathrm{H}_{2}$-stabilized lasers. $\cdots$

I Dose equivalent (PV, 70, 205)

\section{Recommendation 2}

The International Committee for Weights and Measures,

\section{considering that}

- the current definition of the SI unit of dose equivalent (sievert) includes a factor " $N$ " (product of any other multiplying factors) stipulated by the International Commission on Radiological Protection (ICRP), and

- both the ICRP and the International Commission on Radiation Units and Measurements (ICRU) have decided to delete this factor $N$ as it is no longer deemed to be necessary, and

- the current $\mathrm{SI}$ definition of $H$ including the factor $N$ is causing some confusion,

decides to change the explanation in the brochure "Le Système International d'Unités (SI)" to the following:

The quantity dose equivalent $H$ is the product of the absorbed dose $D$ of ionizing radiation and the dimensionless factor $Q$ (quality factor) defined as a function of linear energy transfer by the ICRU:

$$
H=Q \cdot D .
$$

Thus, for a given radiation, the numerical value of $H$ in joules per kilogram may differ from that of $D$ in joules per kilogram depending on the value of $Q$.

The Committee further decides to maintain the final sentence in the explanation as follows: In order to avoid any risk of confusion between the absorbed dose $D$ and the dose equivalent $H$, the special names for the respective units should be used, that is, the name gray should be used instead of joules per kilogram for the unit of absorbed dose $D$ and the name sievert instead of joules per kilogram for the unit of dose equivalent $H$. 
CIPM, 2003

I Revision of the Mise en Pratique list of recommended radiations (PV, 71, 146 and Metrologia, 2004, 41, 99-100)

\section{Recommendation 1}

The International Committee for Weights and Measures,

considering that

- improved frequency values for radiations of some high-stability cold ion standards already documented in the recommended radiations list have recently become available;

- improved frequency values for the infra-red gas-cell-based optical frequency standard in the optical telecommunications region, already documented in the recommended radiations list, have been determined;

- femtosecond comb-based frequency measurements for certain iodine gas-cell standards on the subsidiary recommended source list have recently been made for the first time, leading to significantly reduced uncertainty;

proposes that the recommended radiation list be revised to include the following:

- updated frequency values for the single trapped ${ }^{88} \mathrm{Sr}^{+}$ion quadrupole transition and the single trapped ${ }^{171} \mathrm{Yb}^{+}$octupole transition;

- an updated frequency value for the $\mathrm{C}_{2} \mathrm{H}_{2}$-stabilized standard at $1.54 \mu \mathrm{m}$;

- updated frequency values for the $\mathrm{I}_{2}$-stabilized standards at $543 \mathrm{~nm}$ and $515 \mathrm{~nm}$.

\section{2nd CGPM, 2003}

I Symbol for the decimal marker (CR, 381 and Metrologia, 2004, 41, 104)

\section{Resolution 10}

The 22nd General Conference,

\section{considering that}

- a principal purpose of the International System of Units $(\mathrm{SI})$ is to enable values of quantities to be expressed in a manner that can be readily understood throughout the world,

- the value of a quantity is normally expressed as a number times a unit,

- often the number in the expression of the value of a quantity contains multiple digits with an integral part and a decimal part,

- in Resolution 7 of the 9th General Conference, 1948, it is stated that "In numbers, the comma (French practice) or the dot (British practice) is used only to separate the integral part of numbers from the decimal part",

- following a decision of the International Committee made at its 86th meeting (1997), the International Bureau of Weights and Measures now uses the dot (point on the line) as the decimal marker in all the English language versions of its publications, including the English text of the SI Brochure (the definitive international reference on the $\mathrm{SI}$ ), with the comma (on the line) remaining the decimal marker in all of its French language publications,

- however, some international bodies use the comma on the line as the decimal marker in their English language documents,

- furthermore, some international bodies, including some international standards organizations, specify the decimal marker to be the comma on the line in all languages,
Further updates are available on the BIPM website at www.bipm.org/en/ publications/mep.html. 
- the prescription of the comma on the line as the decimal marker is in many languages in conflict with the customary usage of the point on the line as the decimal marker in those languages,

- in some languages that are native to more than one country, either the point on the line or the comma on the line is used as the decimal marker depending on the country, while in some countries with more than one native language, either the point on the line or comma on the line is used depending on the language,

declares that the symbol for the decimal marker shall be either the point on the line or the comma on the line,

reaffirms that "Numbers may be divided in groups of three in order to facilitate reading; neither dots nor commas are ever inserted in the spaces between groups", as stated in Resolution 7 of the 9th CGPM, 1948.

\section{CIPM, 2005}

I Clarification of the definition of the kelvin, unit of thermodynamic temperature (PV, 94, in press and Metrologia, 2006, 43, 177-178)

\section{Recommendation 2}

The International Committee for Weights and Measures (CIPM), considering

- that the kelvin, unit of thermodynamic temperature, is defined as the fraction $1 / 273.16$ of the thermodynamic temperature of the triple point of water,

- that the temperature of the triple point depends on the relative amount of isotopes of hydrogen and oxygen present in the sample of water used,

- that this effect is now one of the major sources of the observed variability between different realizations of the water triple point,

\section{decides}

- that the definition of the kelvin refer to water of a specified isotopic composition,

- that this composition be:

0.00015576 mole of ${ }^{2} \mathrm{H}$ per mole of ${ }^{1} \mathrm{H}$,

0.0003799 mole of ${ }^{17} \mathrm{O}$ per mole of ${ }^{16} \mathrm{O}$, and

0.0020052 mole of ${ }^{18} \mathrm{O}$ per mole of ${ }^{16} \mathrm{O}$,

which is the composition of the International Atomic Energy Agency reference material Vienna Standard Mean Ocean Water (VSMOW), as recommended by IUPAC in "Atomic Weights of the Elements: Review 2000".

- that this composition be stated in a note attached to the definition of the kelvin in the SI brochure as follows:

"This definition refers to water having the isotopic composition defined exactly by the following amount of substance ratios: 0.00015576 mole of ${ }^{2} \mathrm{H}$ per mole of ${ }^{1} \mathrm{H}$, 0.0003799 mole of ${ }^{17} \mathrm{O}$ per mole of ${ }^{16} \mathrm{O}$ and 0.0020052 mole of ${ }^{18} \mathrm{O}$ per mole of ${ }^{16} \mathrm{O}$ ". 
1 Revision of the Mise en pratique list of recommended radiations (PV, 94, in press and Metrologia, 2006, 43, 178)

\section{Recommendation 3}

The International Committee for Weights and Measures (CIPM),

considering that:

- improved frequency values for radiations of some high-stability cold ion and cold atom standards already documented in the recommended radiations list have recently become available;

- improved frequency values for the infra-red gas-cell-based optical frequency standard in the optical telecommunications region, already documented in the recommended radiations list, have been determined;

- improved frequency values for certain iodine gas-cell standard, already documented in the subsidiary recommended source list, have been determined;

- frequencies of new cold atoms, of atoms in the near-infrared region and of molecules in the optical telecommunications region have been determined by femtosecond combbased frequency measurements for the first time;

decides that the list of recommended radiations be revised to include the following:

- updated frequency values for the single trapped ${ }^{88} \mathrm{Sr}^{+}$ion quadrupole transition, the single trapped ${ }^{199} \mathrm{Hg}^{+}$quadrupole transition and the single trapped ${ }^{171} \mathrm{Yb}^{+}$quadrupole transition;

- an updated frequency value for the $\mathrm{Ca}$ atom transition;

- an updated frequency value for the $\mathrm{C}_{2} \mathrm{H}_{2}$-stabilized standard at $1.54 \mu \mathrm{m}$;

- an updated frequency value for the $\mathrm{I}_{2}$-stabilized standard at $515 \mathrm{~nm}$;

- the addition of the ${ }^{87} \mathrm{Sr}$ atom transition at $698 \mathrm{~nm}$;

- the addition of the ${ }^{87} \mathrm{Rb}$ atom two-photon transitions at $760 \mathrm{~nm}$;

- the addition of the ${ }^{12} \mathrm{C}_{2} \mathrm{H}_{2} \quad(v 1+v 3)$ band and the ${ }^{13} \mathrm{C}_{2} \mathrm{H}_{2} \quad(v 1+v 3)$ and $(v 1+v 3+v 4+v 5)$ bands at $1.54 \mu \mathrm{m}$. 
Appendix 2. Practical realization of the definitions of some important units

Appendix 2 is published in electronic form only, and is available on the BIPM website at www.bipm.org/en/si/si_brochure/appendix2/. 


\section{Appendix 3. Units for photochemical and photobiological quantities}

Optical radiation is able to cause chemical changes in certain living or non-living materials: this property is called actinism, and radiation capable of causing such changes is referred to as actinic radiation. Actinic radiation has the fundamental characteristic that, at the molecular level, one photon interacts with one molecule to alter or break the molecule into new molecular species. It is therefore possible to define specific photochemical or photobiological quantities in terms of the result of optical radiation on the associated chemical or biological receptors.

In the field of metrology, the only photobiological quantity which has been formally defined for measurement in the SI is for the interaction of light with the human eye in vision. An SI base unit, the candela, has been defined for this important photobiological quantity. Several other photometric quantities with units derived from the candela have also been defined (such as the lumen and the lux, see Table 3 in Chapter 2, p. 118).

\section{Actinic action spectrum}

Optical radiation can be characterized by its spectral power distribution. The mechanisms by which optical radiation is absorbed by chemical or biological systems are usually complicated, and are always wavelength (or frequency) dependent. For metrological purposes, however, the complexities of the absorption mechanisms can be ignored, and the actinic effect is characterized simply by an actinic action spectrum linking the photochemical or the photobiological response to the incident radiation. This actinic action spectrum describes the relative effectiveness of monochromatic optical radiation at wavelength $\lambda$ to elicit a given actinic response. It is given in relative values, normalized to 1 for the maximum of efficacy. Usually actinic action spectra are defined and recommended by international scientific or standardizing organizations.

For vision, two action spectra have been defined by the CIE and endorsed by the CIPM: $V(\lambda)$ for photopic vision and $V^{\prime}(\lambda)$ for scotopic vision. These are used in the measurement of photometric quantities and are an implicit part of the definition of the SI unit for photometry, the candela. Photopic vision is detected by the cones on the retina of the eye, which are sensitive to a high level of luminance $\left(L>\mathrm{ca} .10 \mathrm{~cd} \mathrm{~m}^{-2}\right)$ and are used in daytime vision. Scotopic vision is detected by the rods of the retina, which are sensitive to low level luminance $\left(L<\mathrm{ca} .10^{-3} \mathrm{~cd} \mathrm{~m}^{-2}\right)$, used in night vision. In the domain between these levels of luminance both cones and rods are used, and this is described as mesopic vision.

Other action spectra for other actinic effects have also been defined by the CIE, such as the erythemal (skin reddening) action spectrum for ultraviolet radiation, but these have not been given any special status within the SI.
The definition of photometric quantities and units can be found in the International Lighting Vocabulary, CIE publication 17.4 (1987) or in the International Electrotechnical Vocabulary, IEC publication 50 , chapter 845: lighting.

Principles governing photometry, Monographie BIPM, 1983, 32 pp. 


\section{Measurement of photochemical or photobiological quantities and their corresponding units}

The photometric quantities and photometric units which are used at present for vision are well established and have been widely used for a long time. They are not affected by the following rules. For all other photochemical and photobiological quantities the following rules shall be applied for defining the units to be used.

A photochemical or photobiological quantity is defined in purely physical terms as the quantity derived from the corresponding radiant quantity by evaluating the radiation according to its action upon a selective receptor, the spectral sensitivity of which is defined by the actinic action spectrum of the photochemical or photobiological effect considered. The quantity is given by the integral over wavelength of the spectral distribution of the radiant quantity weighted by the appropriate actinic action spectrum. The use of integrals implicitly assumes a law of arithmetic additivity for actinic quantities, although such a law is not perfectly obeyed by actual actinic effects. The action spectrum is a relative quantity; it is dimensionless, with the SI unit one. The radiant quantity has the radiometric unit corresponding to that quantity. Thus, following the rule for obtaining the SI unit for a derived quantity, the unit of the photochemical or photobiological quantity is the radiometric unit of the corresponding radiant quantity. When giving a quantitative value, it is essential to specify whether a radiometric or actinic quantity is intended as the unit is the same. If an actinic effect exists in several action spectra, the action spectrum used for measurement has to be clearly specified.

This method of defining the units to be used for photochemical or photobiological quantities has been recommended by the Consultative Committee for Photometry and Radiometry at its 9th meeting in 1977.

As an example, the erythemal effective irradiance $E_{\text {er }}$ from a source of ultraviolet radiation is obtained by weighting the spectral irradiance of the radiation at wavelength $\lambda$ by the effectiveness of radiation at this wavelength to cause an erythema, and summing over all wavelengths present in the source spectrum. This can be expressed mathematically as:

$$
E_{\text {er }}=\int E_{\lambda} s_{\text {er }}(\lambda) \mathrm{d} \lambda
$$

where $E_{\lambda}$ is the spectral irradiance at wavelength $\lambda$ (usually reported in the SI unit $\left.\mathrm{W} \mathrm{m} \mathrm{mm}^{-1}\right)$, and $s_{\mathrm{er}}(\lambda)$ is the actinic spectrum normalized to 1 at its maximum spectral value. The erythemal irradiance $E_{\text {er }}$ determined in this way is usually quoted in the SI unit $\mathrm{W} \mathrm{m}^{-2}$. 


\section{List of acronyms used in the present volume}

1 Acronyms for laboratories, committees and conferences*

\begin{tabular}{|c|c|}
\hline BAAS & British Association for the Advancement of Science \\
\hline $\mathrm{BIH}$ & Bureau International de l'Heure \\
\hline BIPM & International Bureau of Weights and Measures/Bureau International des \\
\hline & Poids et Mesures \\
\hline CARICOM & Carribean Community \\
\hline CCAUV & $\begin{array}{l}\text { Consultative Committee for Acoustics, Ultrasound and Vibration/ } \\
\text { Comité Consultatif de l'Acoustique, des Ultrasons et des Vibrations }\end{array}$ \\
\hline CCDS* & $\begin{array}{l}\text { Consultative Committee for the Definition of the Second/ } \\
\text { Comité Consultatif pour la Définition de la Seconde, see CCTF }\end{array}$ \\
\hline $\mathrm{CCE}^{*}$ & $\begin{array}{l}\text { Consultative Committee for Electricity/Comité Consultatif d'Électricité, } \\
\text { see CCEM }\end{array}$ \\
\hline CCEM & $\begin{array}{l}\text { (formerly the CCE) Consultative Committee for Electricity and } \\
\text { Magnetism/Comité Consultatif d'Électricité et Magnétisme }\end{array}$ \\
\hline CCL & Consultative Committee for Length/Comité Consultatif des Longueurs \\
\hline $\mathrm{CCM}$ & $\begin{array}{l}\text { Consultative Committee for Mass and Related Quantities/ } \\
\text { Comité Consultatif pour la Masse et les Grandeurs Apparentées }\end{array}$ \\
\hline CCPR & $\begin{array}{l}\text { Consultative Committee for Photometry and Radiometry/ } \\
\text { Comité Consultatif de Photométrie et Radiométrie }\end{array}$ \\
\hline CCQM & $\begin{array}{l}\text { Consultative Committee for Amount of Substance: Metrology in } \\
\text { Chemistry/Comité Consultatif pour la Quantité de Matière : Métrologie } \\
\text { en Chimie }\end{array}$ \\
\hline CCRI & $\begin{array}{l}\text { Consultative Committee for Ionizing Radiation/Comité Consultatif des } \\
\text { Rayonnements Ionisants }\end{array}$ \\
\hline $\mathrm{CCT}$ & $\begin{array}{l}\text { Consultative Committee for Thermometry/Comité Consultatif de } \\
\text { Thermométrie }\end{array}$ \\
\hline CCTF & $\begin{array}{l}\text { (formerly the CCDS) Consultative Committee for Time and Frequency/ } \\
\text { Comité Consultatif du Temps et des Fréquences }\end{array}$ \\
\hline $\mathrm{CCU}$ & Consultative Committee for Units/Comité Consultatif des Unités \\
\hline CGPM & $\begin{array}{l}\text { General Conference on Weights and Measures/Conférence Générale des } \\
\text { Poids et Mesures }\end{array}$ \\
\hline $\mathrm{CIE}$ & $\begin{array}{l}\text { International Commission on Illumination/Commission Internationale } \\
\text { de l'Éclairage }\end{array}$ \\
\hline CIPM & $\begin{array}{l}\text { International Committee for Weights and Measures/ } \\
\text { Comité International des Poids et Mesures }\end{array}$ \\
\hline CODATA & Committee on Data for Science and Technology \\
\hline $\mathrm{CR}$ & $\begin{array}{l}\text { Comptes Rendus of the Conférence Générale des Poids et Mesures, } \\
\text { CGPM }\end{array}$ \\
\hline IAU & International Astronomical Union \\
\hline ICRP & International Commission on Radiological Protection \\
\hline
\end{tabular}

* Organizations marked with an asterisk either no longer exist or operate under a different acronym. 
ICRU International Commission on Radiation Units and Measurements

IEC International Electrotechnical Commission/Commission

IERS International Earth Rotation and Reference Systems Service

ISO International Organization for Standardization

IUPAC International Union of Pure and Applied Chemistry

IUPAP International Union of Pure and Applied Physics

OIML Organisation Internationale de Métrologie Légale

PV Procès-Verbaux of the Comité International des Poids et Mesures, CIPM

SUNAMCO Commission for Symbols, Units, Nomenclature, Atomic Masses and Fundamental Constants, IUPAP

WHO World Health Organization

\section{$2 \quad$ Acronyms for scientific terms}

CGS Three-dimensional coherent system of units based on the three mechanical units centimetre, gram and second

EPT-76 Provisional Low Temperature Scale of 1976/Échelle provisoire de température de 1976

IPTS-68 International Practical Temperature Scale of 1968

ITS-90 International Temperature Scale of 1990

MKS System of units based on the three mechanical units metre, kilogram, and second

MKSA Four-dimensional system of units based on the metre, kilogram, second, and the ampere

SI International System of Units/Système International d'Unités

TAI International Atomic Time/Temps Atomique International

TCG Geocentric Coordinated Time/Temps-coordonnée Géocentrique

TT Terrestrial Time

UTC Coordinated Universal Time

VSMOW Vienna Standard Mean Ocean Water 


\section{Index}

Numbers in boldface indicate the pages where the definitions of the units, or terms, are to be found.

\section{A}

acceleration due to gravity, standard value of

$\left(g_{n}\right), 143$

absolute units, 113

absorbed dose, 107, 118-120, 157, 159-161,

168

actinic radiation, 107, 173-174

actinism, 107, 173

activity referred to a radionuclide, 118,152

amount of substance, 103-105, 114-115, 156-

157

ampere (A), 104, 109-110, 113, 116, 144,

$146,147,149,150$

arcsecond, 124

astronomical unit, 125-126

atomic physics, 125

atomic units, 125-126

atomic weight, 114

Avogadro constant, 115, 125

\section{B}

bar, 127,146

barn, 127-128

base quantity, 103-105, 116

base unit(s), 103-104, 111-116, 147, 148, 149, 156-157

becquerel (Bq), 118, 120, 152, 157

bel (B), 127-128, 134

biological quantities, 107

Bohr radius, bohr, 125-126

British Association for the Advancement of Science (BAAS), 109

\section{C}

calorie, 146

candela (cd), 104, 110, 115-116, 146, 147, 149, 154, 158; new candle, 155

Celsius temperature, 114, 118, 133, 145
CGS, 109, 128-129, 145

CGS-EMU, 105, 128

CGS-ESU, 105, 128

CGS-Gaussian, 105, 128

clinical chemistry, 115, 165

CODATA, 126, 166

coherent derived units, 106, 116-120, 166

Convention du Mètre, 108-109

Coordinated Universal Time (UTC), 157

coulomb (C), 118, 144, 146, 150

Coulomb law, 104

counting quantities, 105,120

curie (Ci), 152

\section{D}

dalton (Da), 125-126

day (d), 122, 124

decibel (dB), 127-128, 134

decimal marker, 102, 133, 169-170

decimal metric system, 108

definitions of base units, 111-116

degree Celsius $\left({ }^{\circ} \mathrm{C}\right), 114,118,131,133,145$, 146

derived quantity, 103, 105, 116-120

derived unit(s), 103, 116-120, 154

digits in threes, grouping digits, 133, 169-170

dimensional symbols, 105

dimensionless quantities, 105, 117, 120, 134, 159

dose equivalent, see sievert

dynamic viscosity (poise), 128, 146

dyne (dyn), 128, 146

\section{E}

electric current, 104-105, 113, 116, 144, 146, 147,149

electrical units, 144

electromagnetic quantities, 104, 128-129 
electron mass, $125-126$

electronvolt (eV), 125-126

elementary charge, 125-126

erg, 128, 146

establishment of the SI, 145, 147, 148, 149

\section{$\mathbf{F}$}

farad $(\mathrm{F}), 118,144,146,150$

foot, 129

formatting the value of a quantity, 133

four-quantity electromagnetic equations, 104

\section{G}

gal (Gal), 128

Gauss, 109

gauss (G), 128

general relativity, 107, 167

Giorgi, 109

gon, 124

$\operatorname{grad}, 124$

gram, 106, 109, 122, 146, 152

gram-atom, gram-molecule, 114

gray (Gy), 118, 120, 157-158, 161

\section{H}

Hall effect (incl. quantum Hall effect), 111, 161-163, 166

Hartree energy, hartree, 125, 126

heat capacity, 119, 131

hectare (ha), 124

henry $(\mathrm{H}), 118, \mathbf{1 4 4}, 146,150$

hertz (Hz), 118, 146, 150

historical note, 108-110

hour (h), 122, 124, 146

hyperfine splitting of the caesium atom, 113

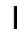

IEC Standard 60027, 104

inch, 129

International Atomic Time (TAI), 155, 156

international prototype of the kilogram, 109,

112, 142, 143

international prototype of the metre, 109, 112,

142, 143, 148

International System of Quantities (ISQ), 104, International System of Units (SI), 104, 145,

$147,148,149$
International Temperature Scale of 1990

(ITS-90), 163-164

International Units (IU) WHO, 108

ionizing radiation, 107, 120, 157-158, 159,

161,168

ISO Standard 31, 102, 104, 131

ISO/IEC Standard 80000, 104

ISO/TC 12, 104, 159

IUPAC, 114-115; Green Book, 131

IUPAP SUNAMCO, 114-115; Red Book, 131

\section{$J$}

Josephson effect, 162

Josephson constant $\left(K_{\mathrm{J}}, K_{\mathrm{J}-90}\right), 162$

joule (J), 106, 118, 119, 131, 144-145, 150

K

katal (kat), 118, 165-166

kelvin (K), 104, 110, 113-114, 116, 153, 154, 170

kibibyte (kilobyte), 121

kilogram, 104, 108-109, 112, 116, 122, 142, $143,147,149,152,165$

kinematic viscosity (stokes), 128

$\mathbf{L}$

legislation on units, 108

length, 104-105, 109, 112, 116, 142, 143, 147

litre (L or 1), 124, 130, 142, 146, 150, 151, 152,159

logarithmic ratio quantities, 127

logarithmic ratio units, 127-128, 134

lumen $(\operatorname{lm}), 118,146,150$; new lumen, 143

luminous intensity, 104-105, 115-116, 143,

$147,149,154,158$

lux (lx), 118, 146, 150

M

magnetic constant, permeability of vacuum, 104,113

mandatory symbols for units, 105, 116, 130131

mass, 104-105, 109, 112, 116, 122, 142, 143, $149,152,165$

mass and weight, 143

Maxwell, 109

maxwell (Mx), 128

mesopic, 158, 173 
metre (m), 104, 108, 112, 131, 142, 143, 146, $147,148,149,160-161$

metric ton, 124, 146

microarcsecond ( $\mu$ as), 122, 124

milliarcsecond (mas), 122, 124

millimetre of mercury, 127

minute (min), 124

MKS system, 109, 144

MKSA system, 109

mole (mol), 104, 110, 114-115, 156-157

molecular weight, 114-115

multiples (and submultiples) of the kilogram, $106,122,152$

multiples, prefixes for, 106, 121-122, 149, $152,155,158,164$

\section{$\mathbf{N}$}

natural units, 125-126

nautical mile, 124, 127

neper (Np), 127-128, 134

newton $(\mathrm{N}), 113,118,144,146,150$

non-SI units, 123-129

numerical value of a quantity, 131-132

\section{0}

œrsted (Oe), 128

ohm $(\Omega), 109,113,118,130,144,146,150$, 161-162, 163, 166

OIML, 108

pascal $(\mathrm{Pa}), 118,131,156$

percent, 134-135

phot (ph), 128

photobiological quantities, 107, 173-174

photochemical quantities, 107, 173-174

photometric units, 143, 154, 173-174

photopic vision, 158, 173

poise $(\mathrm{P}), 128,146$

ppb, 134

ppm, 134-135

ppt, 134

practical units, 109, 145, 147, 148, 149

prefixes, 106, 117, 121, 124, 127-128, 130, $149,152,155,158,164$
Q

quantities of dimension one, 105, 117, 118,

$120,134-135$

quantity, 103

quantity calculus, 131-132

quantity symbols, 105, 131, 132-133

quantity, base, 103, 104, 105, 116

quantity, derived, 103, 104, 105, 116-120

$\mathbf{R}$

radian (rad), 118, 120, 134, 149, 159-160,

164-165

radiation therapy, 107

rationalizing factors, 105

realization of a unit, 101, 111, 172

recommended symbols for quantities, 105 ,

131

reduced Planck constant, 125, 126

\section{$\mathbf{S}$}

scotopic, 158, 173

second (s), 104, 109, 112-113, 116, 131, 146,

147, 148-149, 153

SI prefixes, 106, 117, 121, 124, 127-128, 130, $149,152,155,158,164$

SI, see Système International d'Unités siemens (S), 118, 156

sievert (Sv), 118, 120, 159, 161, 168

sound, units for, 107

special names and symbols for units, 106,

117-120

speed of light in vacuum, 112, 126, 167

standard atmosphere, 127, 147

steradian (sr), 118, 120, 134, 149, 159-160,

164-165

stilb (sb), 128, 146

stokes (St), 128

submultiples, prefixes for, 106, 121-122, 149, $152,155,158,164$

supplementary units, 149, 155, 159-160, 164165

Système International d'Unités (SI), see

International System of Units 
$\mathbf{T}$

TAI, see International Atomic Time tesla $(\mathrm{T}), 118,150$

thermodynamic temperature, 104-105, 113-

114, 146, 147, 149, 153, 154, 170

thermodynamic temperature scale, 146

Thomson, 109

time (duration), 104-105, 112-113, 116, 147, 153

tonne, 124,146

triple point of water, 113-114, 144-145, 146, 154,170

U

uncertainty, 133

unit (SI), 111-120

unit names, 131, 146

unit symbols, 116, 130, 146

unit, base, 103, 111, 116, 147, 149, 157

unit, derived, 103, 116-119, 150, 154

units for biological quantities, 107

UTC, see Coordinated Universal Time
V

value of a quantity, 131-132

volt (V), 118, 144, 146, 150, 161, 162

von Klitzing constant $\left(R_{\mathrm{K}}, R_{\mathrm{K}-90}\right), 111,163$, 166

W

water, isotopic composition, 114, 170

watt (W), 118, 144, 146, 150

Weber, 109

weber (Wb), 118, 144, 150

WHO, 108

\section{$\mathbf{Y}$}

yard, 129

STEDI MEDIA

1, Boulevard Ney, 75018 Paris

Dépôt légal, $n^{\circ} 8844$

ISBN 92-822-2213-6

Achevé d'imprimer : mai 2006

Imprimé en France 